\title{
Creation of fibrous plant protein foods
}

Birgit L. Dekkers 


\section{Thesis committee}

\section{Promotors}

Prof. Dr A.J. van der Goot

Personal chair at Food Process Engineering

Wageningen University \& Research

Prof. Dr R.M. Boom

Professor of Food Process Engineering

Wageningen University \& Research

\section{Other members}

Prof. Dr A. Mathys, ETH Zürich, Switzerland

Prof. Dr L. Ahrné, University of Copenhagen, Denmark

Dr M. Mellema, Unilever, Vlaardingen, The Netherlands

Prof. Dr E. van der Linden, Wageningen University \& Research

This research was conducted under the auspices of the Graduate School VLAG (Advanced studies in Food Technology, Agrobiotechnology, Nutrition and Health Sciences). 


\title{
Creation of fibrous plant protein foods
}

\author{
Birgit L. Dekkers
}

Thesis

submitted in fulfillment of the requirements for the degree of doctor

at Wageningen University

by the authority of the Rector Magnificus,

Prof. Dr A.P.J. Mol,

in the presence of the

Thesis Committee appointed by the Academic Board

to be defended in public

on Friday 14 September 2018

at 1.30 p.m. in the Aula. 
Birgit L. Dekkers

Creation of fibrous plant protein foods

206 pages.

PhD thesis, Wageningen University, Wageningen, the Netherlands (2018)

With references, with summary in English

ISBN 978-94-6343-319-8

DOI $10.18174 / 456158$ 


\section{Content}

$\begin{array}{ll}\text { Chapter } 1 & 1\end{array}$

Introduction

Chapter 2

A combined NMR approach for determining water distribution in protein blends

\section{Chapter 3}

The phase properties of soy protein and wheat gluten in a blend for fibrous structure formation

\section{Chapter 4}

Shear-induced fibrous structure formation from a soy protein isolate - pectin blend

\section{Chapter 5}

Understanding fiber formation in a concentrated soy protein isolate - pectin blend

\section{Chapter 6}

Viscoelastic properties of soy protein isolate - pectin blends

\section{Chapter 7}

Aqueous fractionation processes of soy protein for fibrous structure formation

\section{Chapter 8}

Structuring processes for fibrous plant protein products

\section{Chapter 9}

General discussion

\section{References}

\section{Summary}

Acknowledgements

About the author

199

List of publications

201

Overview of completed training activities 



\section{Chapter 1}

Introduction 


\subsection{Why meat analogues?}

Meat is an important diet component in many food cultures, and main meals are often composed around this protein source (Sui, Raubenheimer, \& Rangan, 2017). Meat, when consumed in low or moderate quantity, is nutritious, because it has a high protein content, both in quality and in quantity, and contains micronutrients such as iron and vitamin B12 (Biesalski, 2005). However, there are several reasons for arguing that meat production and consumption should be lowered. A transition from animal to plant based protein is required to guarantee the production of sufficient protein for all, and at the same time mitigate climate change. The production of meat imposes a burden on the environment in terms of greenhouse gas emission, energy consumption, land and water use, and inefficient use of resources (Nijdam, Rood, \& Westhoek, 2012). Furthermore, there are concerns related to the health effects of meat consumption. Short-term health effects concern the presence of bacteria, such as Escherichia coli and Salmonella enterica, resulting in food poisoning. The prevalence of resistant bacteria has been recognized as a critically important global public health thread (Schmidt et al., 2015). Other, long term negative health effects concern the relation between high red meat consumption and colon cancer, and the use of hormones in meat production, which has been related to increased risk of breast cancer in women (Galbraith, 2002; McAfee et al., 2010; Micha, Wallace, \& Mozaffarian, 2010). Besides, ethical aspects with regards to animal welfare are used by some as a reason to reduce meat production and consumption.

Despite the concerns described above, there is only a fraction of the world population that adopts a vegetarian diet (Stiftung, Böll, \& Terre, 2014). The reason for this is that meat is perceived as tasty, healthy and nutritious by consumers (Schösler, de Boer, \& Boersema, 2012; Verbeke et al., 2010). To help consumers to lower their meat consumption, several strategies have been employed (Laestadius, Neff, Barry, \& Frattaroli, 2013). One strategy is actively trying to change the behaviour of consumers by encouraging 'meatless days' or 'meatless weeks', and replacement of meat by plant-based proteins in the form of vegetables, beans, pulses, or nuts (De Boer, Schösler, \& Aiking, 2014). Another strategy is developing products that can directly replace meat in a meal context. These products are often structured foods made from plantbased ingredients. Traditional products, such as tofu and tempeh, are examples of structured foods that have been mainstream foods in East Asia for centuries. The acceptance of these types of products is, however, lower in Western countries (Asgar, Fazilah, Huda, Bhat, \& Karim, 2010). Therefore, another category of structured foods has been developed that matches better 
to the Western diet and current food culture: meat analogues. Meat analogues are products that resemble meat in its functionality, and are prepared by the consumer as if they were meat. Products that approach the original meat product best are considered to be the most promising to reduce meat consumption for the largest group of consumers (Elzerman, Hoek, van Boekel, \& Luning, 2011). Therefore, meat analogues should resemble meat in terms of their sensory properties, unique texture and taste, since these are key properties appreciated in meat by consumers (Grunert, Bredahl, \& Brunsø, 2004).

\subsection{The structure of meat and meat analogues}

Meat analogues mimic meat muscle structure. The unique texture of meat is the result of the hierarchical structural organization of the muscle. Muscle consists of meat fibres (Pearson, 2012; Pette \& Staron, 1990). These muscle fibres are surrounded and supported by connective tissue, consisting mostly of collagen and elastin. Muscle fibres are independent structures varying in length between 10 and $40 \mathrm{~mm}$ in length and 10 to $100 \mu \mathrm{m}$ in diameter. These muscle fibres are a collection of smaller subunits, myofibrils, which contain a number of smaller long thin filaments: the myofilaments. Myofilaments are constructed from sarcomeres, which are composed of the proteins myosin, forming thick filaments (4 - $16 \mathrm{~nm}$ in diameter), and actin, forming thin filaments $(6-8 \mathrm{~nm}$ in diameter). The quality and sensory attributes of meat are often related to these structural elements (Damez \& Clerjon, 2008, 2013). For example, the toughness of meat is related to the distribution of myofibrillar and connective tissues. Meat tenderness is strongly related to the spatial organization of the connective tissue throughout the meat fiber bundles. Furthermore, the water held between meat fibres is connected to the juiciness of meat.

Meat analogues, which mimic the fibrousness similar to meat muscle, can be obtained with plantbased proteins that are aligned by applying shear. This principle is used in extrusion, which is the most commonly used commercial technique to create fibrous products. In a twin-screw extruder, plant-based ingredients are structured by intensive heating and mixing in the barrel. In low moisture extrusion, slightly expanded products are obtained that have to be hydrated afterwards to create a meat-like end-product. In high moisture extrusion, the hydrated ingredients flow into a cooling die, preventing expansion. Structure formation takes place during cooling as a result of lamellar shear flow (Cheftel, Kitagawa, \& Queguiner, 1992; Seiichiro \& Noguchi, 1987). Another technique for creating meat-like products is mixing proteins and hydrocolloids that coagulate with cations, ensuring the entrapment of the anisotropic structures 
(Kweldam, 2011). A decade ago, a technology based on simple shear flow deformation was introduced to create meat-like products, referred to as the shear cell technology. Similarly to extrusion, biopolymers can be heated while shearing.

\subsection{Shear cell technology}

The behaviour of (bio)polymers under deformation has been studied extensively, but generally with conventional rheometers (Ako, Durand, \& Nicolai, 2011; Stokes, Wolf, \& Frith, 2001). The behaviour of (bio)polymers under shearing deformation is less complex as compared to the very complex and sometimes even chaotic flow patterns during other structuring processes such as extrusion and mixing. Further, biopolymers are often studied in lower concentrations, due to better defined behaviour and the lower forces required to deform such solution. It allows precise measuring of both deformation and stresses. However, meat analogue products require higher biopolymer concentrations and have to be heated above the boiling temperature of water, which cannot be assessed with conventional rheometers. Therefore, so-called shear cells were developed, which can apply very high shear stresses and high temperatures, and which were shown to yield very well structured materials with a range of proteins (Manski, van der Goot, \& Boom, 2007b). These shear cells were not only developed to study the deformation and structuring behaviour of concentrated (bio)polymer systems under simple shear flow, but also for understanding the mechanisms underlying the changes in molecular weight of starch during a heat-shear treatment, and separation of wheat flour into starch and gluten at high concentrations (Peighambardoust, Hamer, Boom, \& van der Goot, 2008; van den Einde, van der Goot, \& Boom, 2003).

Besides the ability to use simple shear flow deformation, there are also several other advantages of using shear cells for research purposes. First, biopolymers can be structured batchwise, which allows process conditions, such as temperature, time and rotation speed, to be varied independently. For this reason, flow (shear) and time-temperature dependency of material can be decoupled and studied separately. Second, the flow field is relatively homogeneous, and process control in a shear cell can be adapted and monitored accurately. Finally, relatively small amounts of plant-based material are required when performing structuring experiments on a labscale, and therefore a larger experimental window can be explored in a given time.

Two device geometries were developed for studying the behaviour of concentrated (bio)polymers under simple shear flow deformation; a cone-in-cone geometry and a couette 
geometry (Fig. 1.1) (Peighambardoust, van Brenk, van der Goot, Hamer, \& Boom, 2007; van den Einde et al., 2003). In a cone-in-cone device, the bottom cone is rotating while the top cone is stationary, and the material placed in between is sheared. During shearing, material can be heated from both sides, and to prevent moisture evaporation, the shear cells are sealed at the rim. The disadvantage of sealing is that it influences the torque values. As a result information obtained by the torque measurement on the state of the material in between the cones should be interpreted carefully. The speed of the rotating bottom cone and the angle between the two cones determines the rate of deformation.
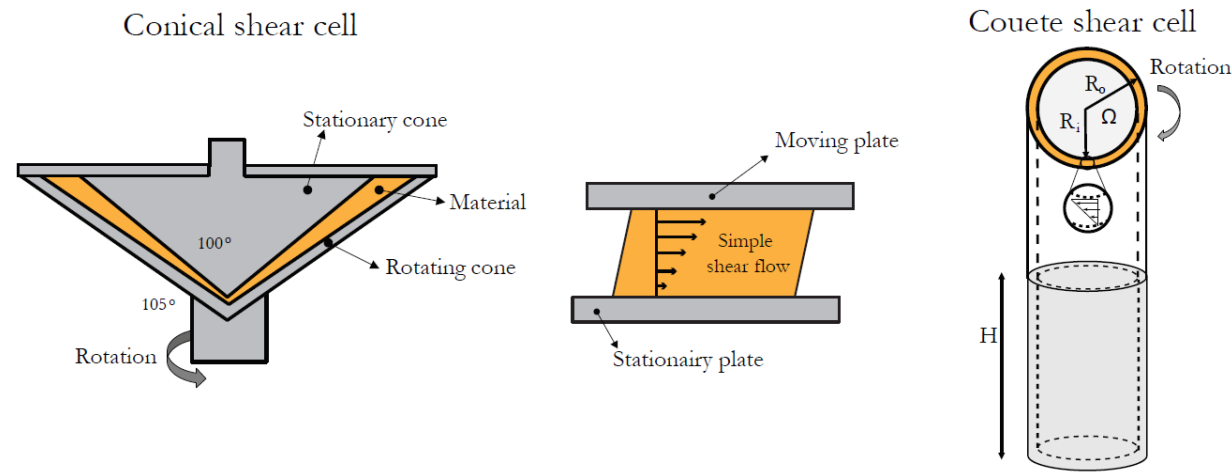

Fig. 1.1 Illustration of cone-in-cone and couette device in which materials can be deformed with simple shear flow

A couette, or cylinder-in-cylinder device consists of a stationary outer cylinder and a smaller rotating inner cylinder. The two concentric cylinders induce Couette flow in the gap, which is to a large degree similar as the flow inside a cone-in-cone device (Krintiras, Göbel, Bouwman, van der Goot, \& Stefanidis, 2014; Peighambardoust et al., 2007). Both devices have been used successfully to structure proteins into fibrous structures for the application of meat analogues.

\subsection{Structure formation with shear cell technology}

Calcium caseinate, a dairy protein, was the first protein that was transformed into a fibrous product with a shear cell (Manski et al., 2007b; Manski, van der Zalm, van der Goot, \& Boom, 2008). Macroscopically fibrous materials were obtained when dense aqueous dispersions of calcium caseinate $\left(30 \mathrm{wt} \%\right.$ ) were sheared at roughly $50 \mathrm{~s}^{-1}$ (Fig. 1.2A). A hierarchical microstructure was observed with oriented fibres having a diameter in the order of $100 \mathrm{~nm}$. The formation of these structures was explained by the alignment of protein aggregates or caseinate micelles due to the shear deformation, and concurrent solidification of the aligned protein aggregates through enzymatic gelation. The tensile strength of the product parallel and 
perpendicular to the shear flow was shown to be an useful method in quantifying the degree of anisotropy, and was thus used an indication for the fibrousness of the materials.

A shear cell has also been used to create anisotropic structures with a fibrous appearance from plant materials. Soy protein isolate (SPI) - wheat gluten (WG) blends were transformed into anisotropic materials when applying rotational speeds of $40 \mathrm{~s}^{-1}$ while heating at $95^{\circ} \mathrm{C}$ for $15 \mathrm{~min}$, and subsequent cooling to room temperature (Fig. 1.2B) (Grabowska, Tekidou, Boom, \& van der Goot, 2014). Depending on the dry matter content and the ratio between SPI and WG, a wide variety of macrostructures can be created: isotropic gels, structures with fibres, and structures with layers and fibres. It was hypothesized that only dense protein systems with a sufficiently high viscosity will allow deformation and alignment required for the formation of an anisotropic material. SPI alone resulted in layered structures only, it was therefore concluded that at least a two-phase system is required to produce an anisotropic structure with a fibrous appearance in a shear cell. This is in accordance with earlier work done on extrusion processing by Cheftel et al., who showed that extrusion of SPI yielded an isotropic material, whereas proteins in combination with wheat gluten or polysaccharides resulted in anisotropic materials (Cheftel et al., 1992).

The importance of a multiphase system for structure formation was also shown for soy protein concentrate (SPC), which is an ingredient that naturally consists of at least two phases; proteins and polysaccharides (Grabowska et al., 2016). The microstructure of extruded SPC revealed that also after structure formation the proteins and polysaccharides clearly formed separate phases. However depending on the process conditions, SPC was transformed into a gel, or in a structure with a layered or fibrous appearance. The heating temperature was shown to be a key process parameter, since shearing while heating at $140^{\circ} \mathrm{C}$ yielded a fibrous morphology (Fig. 1.2C), whereas lower temperatures resulted in a layered morphology. Grabowska et al. optimized the dry matter content and the rotational speed and found that at the optimum conditions the proteins and polysaccharides were deformed and aligned in the shear flow direction. Other
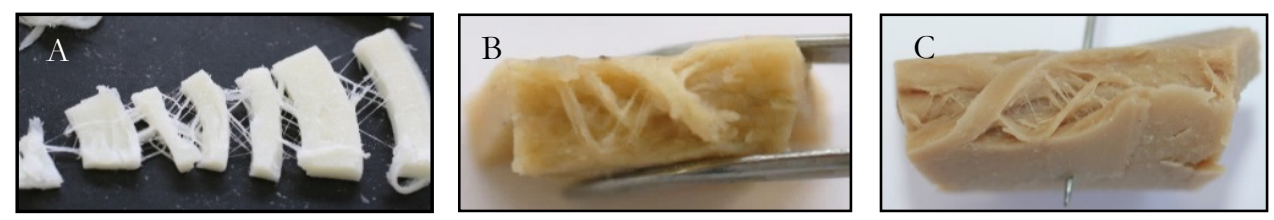

Fig. 1.2 Fibrous macrostructures from A) calcium caseinate, B) soy protein isolate - wheat gluten, and C) soy protein concentrate 
conditions gave hardly any orientation on a microstructural level. Thus it was concluded that for successful fibrous structure formation, ingredients have to be aligned and concurrently solidified. The exact deformation of the phases depends on the interplay between ingredients, their viscoelastic properties and processing conditions.

Although the previous studies identified optima in ingredient combinations and process conditions for specific systems, there is not yet a mechanistic understanding of the product and process dynamics. Such better understanding is essential to extrapolate findings towards the use of different ingredients, or towards other processing conditions. The detailed study of the mechanisms is by no means trivial, since materials involved are already complex mixtures, while both the alignment occurs at high shear stresses, which are beyond the range of normal rheometers. In addition, the temperatures required for structuring are well above $100^{\circ} \mathrm{C}$, and therefore experiments cannot be done at ambient pressure without losing water from the system.

A final aspect relates to the properties of the raw materials. Those depend not only on the plant source, but also on the extraction process applied to produce protein concentrates or isolates. Within this thesis, soy protein is used as the primary raw material to study the structure formation process. Therefore, understanding the properties of soy protein as function of extraction conditions is of crucial importance to develop a robust process for the next generation meat analogues.

\subsection{Aim and outline of the thesis}

The overall aim of this thesis is to obtain insight in the key mechanisms that determine the transformation of plant-based biopolymer blends into anisotropic/fibrous structures with the shear cell technology. A prerequisite for further understanding the structure formation process is the ability to quantitatively analyse the properties of plant ingredients prior to and during processing. However, there were no methods readily available to determine the properties of these plant-based ingredients at the conditions that lead to anisotropic/fibrous structure formation. Therefore, methods were developed to study and quantify these properties at relevant conditions. Part of the methods focussed on describing the changes in physical properties as function of process conditions due to chemical changes inside the proteinaceous phases. Those methods were further explored by varying the process conditions in the shear cell, and the properties of the ingredients prior to structuring. Finally, structure formation was put into a broader perspective by giving an overview of the different processing techniques that were 
currently available to create fibrous structures for the application of meat analogues. The thesis outline is shown in Fig. 1.3.

\begin{tabular}{|c|c|c|}
\hline \multicolumn{2}{|c|}{ Introduction } \\
Method development & Process & Raw materials \\
Chapter 2 & Chapter 4 & Chapter 4 \\
Chapter 3 & Chapter 5 & \\
Chapter 6 & Chapter 7 \\
& Chapter 8 & \\
& General discussion & \\
\hline
\end{tabular}

Fig. 1.3 Thesis outline

In Chapter 2, a methodology is presented to determine the water distribution in soy protein isolate (SPI) - wheat gluten (WG) blends. Time-domain NMR relaxometry (TD-NMR) is used to directly determine the concentration of water in each separate phase. Oscillatory rheology was then used to quantify the rheological properties of the blend as function of the rheological properties of the individual phases and the water distribution in a blend. This method was developed for blends at room temperature and also applied to heated and sheared samples in Chapter 3. Here, the predictions in water distribution based on TD-NMR are used to determine the viscoelastic properties of the SPI and WG phase in the blend, which yields insight in the deformability of the two phases in a blend under those conditions that are relevant for structure formation, i.e. during heating and shearing.

Chapter 4 introduces a model blend that mimics soy protein concentrate (SPC) being built up of a highly pure protein phase, soy protein isolate (SPI), and a soluble, pure polysaccharide phase, pectin. The effect of temperature and the ratio between protein and polysaccharides on the structuring process are investigated by analysing the macrostructure, microstructure and mechanical properties of the resulting fibrous materials. Hypotheses are developed for the origin of the fibrous appearance of these products. Chapter $\mathbf{5}$ extends the study on structure formation with SPI-pectin blends. Here, the deformation of the dispersed pectin phase and also the influence of incorporated air are considered. A model based on the rule of mixing is used to predict the mechanical anisotropy based on the volume fraction and the deformation of the weak, dispersed phase. The degree of deformation is varied by applying various shear rates. 
In Chapter 6, the behaviour of the SPI and pectin phases in a blend is investigated by determining the viscoelastic properties while shearing and heating over time. A closed cavity rheometer (CCR) is used to determine these properties under similar conditions as used during fibrous structure formation. The effects of pectin degradation by the release of galacturonic acid, which reduces the $\mathrm{pH}$ and leads to redistribution of water, are considered to explain the difference between the use of separate ingredients and the blend.

In previous chapters, commercial protein isolates and concentrates were used to create fibrous structures. While these components can give fibrous materials, it is unknown whether these proteins have the ideal properties for fibrous structure formation. Besides, production of those ingredients is associated with the use of organic solvents and other chemicals, which compromises the potential gain in sustainability. That is why we investigated an aqueous fractionation process to obtain soy protein fractions. The aim of this study, which is described in Chapter 7, is to reveal properties of soy protein fractions that are required for fibrous structure formation. Therefore, various soy protein fractions are structured in the shear cell, under conditions that are known to yield fibrous structures from commercial SPC. All fractions, the successful and the failing ones, are further analysed to determine their functional properties.

In Chapter 8, an overview is presented of all the techniques that are commercially used and currently investigated to create fibrous products. The different techniques are compared in terms of their processing approach, potential resemblance of the product to meat, scalability, robustness, and efficiency of the process. The techniques that make use of directed assembly processes in multiphase systems are further investigated by reviewing literature on similar processes used for other applications. These insights are subsequently translated to the conditions as used during structure formation for meat analogues. In addition, the state of the art in analytical methods are described, which are developed to study directed assembly processes during structuring or quantify the anisotropy of products formed using those conditions.

Chapter 9 concludes with a general discussion of all results presented in the thesis. First, the main findings presented are summarized. Then the insight described in all chapters are integrated in design rules for fibrous structure formation. The challenges related to studying complex materials and unconventional process conditions are discussed. Finally, the scientific challenges to resolve prior to commercialization of the shear cell technology are outlined. 


\section{Chapter 2}

\section{A combined NMR approach for determining water distribution in protein blends}

This chapter has been published as B.L. Dekkers ${ }^{1}$, D.W. de Kort ${ }^{1}$, K.J. Grabowska, B. Tian, H. van As, \& A.J. van der Goot (2016). A combined Rheology and Time Domain NMR approach for determining water distribution in Protein Blends. Food Hydrocolloids, 60, 525-532. http://doi.org/10.1017/CBO9781107415324.004

${ }^{1}$ Authors contributed equally 


\section{Abstract}

We present a combined time domain NMR and rheology approach to quantify the water distribution in a phase separated protein blend. The approach forms the basis for a new tool to assess the microstructural properties of phase separated biopolymer blends, making it highly relevant for many food and non-food related applications. First, we determine the relaxation rate of absorbed water, and the viscoelastic properties of the separated phases as function of the water content. Next, the same properties are measured for the protein blends. Finally, predictions for water distribution obtained from rheological experiments are made via the polymer blending law, and compared to a more direct assessment of the water distribution with time-domain NMR relaxometry (TD-NMR). In this study, the protein blend consists of soy protein isolate (SPI) and vital wheat gluten (WG). We demonstrate that predictions for water distribution are similar for both TD-NMR and rheological measurements. It turns out that water does not distribute homogenously over the phases. Independent of the SPI and WG ratio, more water is absorbed by the SPI phase relative to the WG phase, which largely determines the resulting rheological properties of the blends. 


\subsection{Introduction}

Microstructural assessment of biopolymer blends is highly relevant for many food and non-food applications. However, most analytical methods have been developed for more diluted dispersions and are not directly suitable for the characterization of denser materials (Kasapis, 2008). Unfortunately, the properties of a denser system cannot be simply predicted by analysing the properties at lower concentration. For example, the interaction between water and protein is recently described as being concentration dependent (van der Sman, 2012), giving denser proteins blends properties, which cannot be derived from diluted mixtures. It explains the need for the development of new tools for dense-biopolymer materials.

Rheology is often used to characterize the properties of biopolymer blends (Song \& Kim, 2004; van Riemsdijk, Snoeren, van der Goot, Boom, \& Hamer, 2011). However, this method does not provide direct information about the characteristics of the individual phases and the blend microstructure. To obtain some information about the microstructure, the "polymer blending law" can be used to predict whether a protein blend reacts in an isostress, isostrain, or bicontinuous manner with respect to the applied forces. This empirical law was established by Takayanagi (Kasapis, Norton, \& Johan, 2009; Takayanagi, 1963) and is expressed in its general form as:

$$
M^{n}=\phi_{X} M_{X}^{n}+\phi_{Y} M_{Y}^{n}
$$

where $\phi$ denotes the volume fraction of the individual phases and $M$ can be either Young's modulus (E), the shear modulus $(G)$ or the storage modulus ( $\left.G^{\prime}\right)$ from oscillatory measurements. The exponent equals $n=1$ for isostrain, $n=-1$ for isostress in systems in which one component is dispersed in the other (Kasapis, et al., 2009; Morris, 2009), and $n=0.2$ for bicontinuous systems (Davies, 1971; Gilsenan, Richardson, \& Morris, 2003; Piculell, Nilsson, \& Muhrbeck, 1992). The isostress regime is suitable to describe a blend with a dispersed phase with a high viscosity, while the isostrain regime is suitable to describe the behaviour of a dispersion in which the moduli of both phases are similar or the dispersed phase is weaker. In case of bicontinuous systems, it is assumed that both phases form a continuous phase and therefore contribute more equally to the final product properties.

The mechanical properties of a biopolymer blend are strongly influenced by the rheological properties of the phases, which depend on the water content in that phase, and therefore the water distribution in the blends. This fact has already been recognized by various authors (Clark, 
Richardson, Ross-Murphy, \& Stubbs, 1983; Fitzsimons, Mulvihill, \& Morris, 2008; Shrinivas, Kasapis, \& Tongdang, 2009). In those studies, the water distribution, which can be expressed as avidity factor $p$, is taken as a fitted parameter when analysing the rheological properties. Microscopy is then used as qualitative confirmation. Theoretically a quantitative interpretation of microscopy pictures is possible, provided that each phase can be clearly distinguished, for example by using different stains. However, this quantification requires a significant effort (taking many images of a sample to get a relevant overview, stacking of $2 \mathrm{D}$ pictures to get a $3 \mathrm{D}$ overview etc.), and in-depth image analysis, which relies heavily on computer algorithms to recognize, differentiate and quantify images (Aguilera, 2005).

It is clear that the interpretation of rheological data would benefit from the availability of a method for direct and accurate determination of the water distribution in the total sample. Clark (1983) already proposed NMR relaxometry as an adequate analytical tool for this determination (Clark, et al., 1983).

In this work, we therefore focus on the characterization of concentrated, phase separated protein blends. We use an aqueous dispersion consisting of soy protein isolate (SPI) and vital wheat gluten (WG). SPI and WG are assumed to be immiscible at high concentrations and to form separate phases with sizes in the $\sim 100 \mu \mathrm{m}$ range upon shearing and heating. This assumption is based on the fact that especially gluten is unable to completely dissolve in water. It absorbs twice its weight in water at most, giving a soft-solid material with the characteristic viscoelastic (doughlike) behaviour (Grabowska, Tekidou, Boom, \& van der Goot, 2014; Peighambardoust, Hamer, Boom, \& Van der Goot, 2008). Such swollen WG material cannot be mixed at molecular level with other biopolymer materials, which was confirmed by microscopic analysis of a structured SPI-WG blend that revealed clear phase separation (Grabowska, et al., 2014; Krintiras, Diaz, van der Goot, Stankiewicz, \& Stefanidis, 2016; Krintiras, Gobel, Bouwman, van der Goot, \& Stefanidis, 2014). In a phase separated SPI-WG blend, the protein concentration in each phase is related to the water distribution over the two phases. Since the water holding capacities of SPI and WG differ largely, we expect that water will not distribute evenly over the SPI and WG phases, which implies that the volume fractions of the individual components in the SPI-WG blend will not be equal to the mass fractions after equilibration. If water does not distribute homogeneously, one phase would be more diluted, whereas the other would be more concentrated. The actual protein concentration in each phase can be calculated using mass balances as explained in Appendix A. 
Here, we develop an approach based on time domain nuclear magnetic resonance relaxometry (TD-NMR) to assess water content and relaxation rate of absorbed water in the separate phases (Bosmans, et al., 2012; Mariette, 2009; Van As \& van Duynhoven, 2013; Van Duynhoven, Voda, Witek, \& Van As, 2010). Since our model system is phase separated into a WG and a SPI phase, we measure proton transverse relaxation times $T_{2}$ of water in dispersions of the individual SPI and WG proteins and use this as input to obtain estimates for the water distribution (and phase volume fractions) in the SPI-WG blends. Diffusive exchange is expected to be negligible. This is based on the fact that SPI and WG are assumed to be immiscible and to form relatively large separate phases with high viscosity given the density of the blend. Recently, a similar conclusion was drawn for dispersions containing dense whey protein particles (Peters, et al., 2016). The objective of this study is to obtain better insight in the rheological behaviour of SPI-WG dispersions by considering the distribution of water over both phases and to understand the effect of water content on the rheological properties of the individual phases. The results are used to hypothesize on the microstructure in the blends.

\subsection{Materials and methods}

\subsubsection{Materials}

Soy protein isolate (SPI) and vital wheat gluten (WG) were donated by Barentz (Hoofddorp, The Netherlands). Soy protein isolate (Supro EX 37) is a powder containing at least $90 \mathrm{wt. \%}$ protein $(\mathrm{N} \times 6.25),<5 \mathrm{wt} . \%$ ash and $<6 \mathrm{wt} . \%$ water, as indicated by the manufacturer (Solae, St. Louis, Missouri, USA). Vital WG, produced by Roquette (Lestrem, France), contains $>83$ wt. $\%(\mathrm{~N} \times 6.25)$ protein based on the dry matter and 8 wt.\% water, as indicated by the manufacturer. Water binding capacity of soy protein isolate is reported to be $8.9 \mathrm{~g}$ water $/ \mathrm{g}$ dry matter and the water binding capacity of wheat gluten is $1.9 \mathrm{~g}$ water / $\mathrm{g}$ dry matter (Grabowska, et al., 2014). Food-grade sodium chloride $(\mathrm{NaCl})$ was obtained from Sigma-Aldrich (Zwijndrecht, The Netherlands).

\subsubsection{Sample preparation}

Dispersions of SPI (11.0 - 50.0 wt.\%) and WG (20.0 - 60.0 wt.\%), and SPI-WG blends (25.0 40.0 wt.\%) were prepared at different ratios SPI and WG (20/80, 35/65, 50/50, 65/35, 80/20). All samples contained $1.0 \mathrm{wt} . \% \mathrm{NaCl}$. The salt was dissolved in water before adding the solution to the protein powders. $\mathrm{NaCl}$ was added because it is often used in SPI-WG blends, which are structured into fibrous materials for the meat analogue application (Grabowska, et al., 2014). In 
case of the SPI-WG blend, the protein powders were mixed thoroughly before adding the salt solution. The dispersions were then mixed with a spatula. All samples were allowed to equilibrate at room temperature for $10 \mathrm{~min}$ before actual measurement. It must be noted that there was no difference for the rheology and TD-NMR measurements in case protein powders were added to water or water was added to the protein powders or kept for longer times.

\subsection{3 ${ }^{1} \mathrm{H}$ time domain NMR relaxometry}

Samples were transferred into 7-mm NMR tubes that were sealed to prevent water loss during the experiment. All experiments were repeated four to eight times to allow adequate estimation of the experimental error. ${ }^{1} \mathrm{H}$ relaxometry was performed at $0.72 \mathrm{~T}$ magnetic field strength (30.7 MHz ${ }^{1} \mathrm{H}$ resonance frequency) using a Maran Ultra NMR spectrometer, controlled by RINMR software (Resonance Instruments Ltd., Witney, UK). The decay of the transverse magnetization, a process characterized by transverse relaxation time $T_{2}$, was measured in the time domain by means of the Carr-Purcell-Meiboom-Gill (CPMG) experiment. During the CPMG pulse train, 12288 echoes (five data points per echo) were recorded. The sample time between the data points in each echo was $10 \mu$ s resulting in a spectral width of $100 \mathrm{kHz}$. The time between each echo was $407 \mu$ s. In total, 16 transients were recorded with phase cycling resulting in no baseline offset, with a repetition time of $30 \mathrm{~s}$, resulting in a total measurement time of 8 min. Each CPMG echo train was phase corrected and each echo was averaged to one data point using the IDL package (ITT Visual Information Solutions, Boulder, CO, USA).

The decay curves of the transverse magnetization were analysed with the Levenberg-Marquardt non-linear least squares algorithm implemented in SplMod (Vogel, 1988), which fits a sum of exponential curves (“components") $\sum_{\mathrm{i}} \mathrm{A}_{\mathrm{i}} \mathrm{e}^{-\mathrm{t} / T_{2, \mathrm{i}}}$ to the decay, resulting in an amplitude $A$ and transverse relaxation time $T_{2}$ for each component $i$. The inverse of $T_{2}$ is the relaxation rate $R_{2}$. The number of decay components needed to describe the data was estimated from the decrease of the standard deviation when fitting an additional component. For all experiments, we found that the data could be accurately described with four components (justification in Appendix B2).

\subsubsection{Oscillatory measurements}

Oscillatory measurements were performed using a stress-controlled rheometer (Paar MCR 301, Anton Paar, Graz, Austria). A strain sweep test $(0.5$ - 50\%) was done to determine the linear viscoelastic region (LVR) at a frequency of $1 \mathrm{~Hz}$. A frequency sweep was performed at a constant strain of $1.0 \%$ (within the linear part). The frequency was varied from $0.1-10 \mathrm{~Hz}$, and 21 16 
measurements were recorded. Serrated plate-plate geometry (diameter $25 \mathrm{~mm}$ ) was take to reduce risk of slippage and the gap was normal force controlled at $0.1 \mathrm{~N}$ with $0.025 \mathrm{~N}$ hysteresis. All tests were performed at $25^{\circ} \mathrm{C}$ and done in triplicate.

\subsection{Results and discussion}

This section first describes the TD-NMR results of the single phases and the blends. The outcomes of the single phases are used to predict the water distribution in the blends. Then, the rheological properties of the single phases and the blends are presented. The concentration dependence of the moduli is fitted and used to estimate the water distribution inside the protein blends for which the three polymer blending laws are compared. Lastly, we compare the prediction of the water distribution by both methods and hypothesize about the structure of the blend.

\subsection{1 ${ }^{1} \mathrm{H}$ TD-NMR relaxometry for SPI and WG dispersions}

Components with significantly different $T_{2}$ represent populations of protons $\left({ }^{1} \mathrm{H}\right)$ with different rotational mobility, and can therefore be assigned to different groups of proton-bearing moieties in the sample. Four relaxation times $T_{2}$ were needed to describe all CPMG relaxation curves accurately (justification of the number of components in appendix B). If we compare 1, 2, 3 and 4-component fits to a Laplace inversion of the data (by CONTIN (Provencher, 1982), Fig. 2.1), we see that the Laplace inversion predicts three separate $T_{2}$ distributions. A 4-component fit reproduces this picture, where the two middle components together describe the large and slightly dispersed middle population.

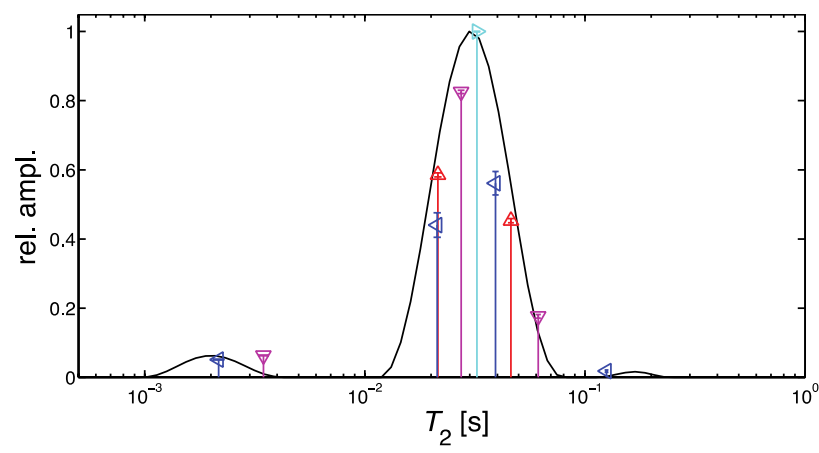

Fig. 2.1 Comparison of Laplace inversion (by CONTIN) and discrete-component fits for a representative data set (SPI 20\%). Black line: Laplace inversion. Coloured bars: $(>$-sign) 1-component fit, $(\triangle$-sign) 2-component fit, $\nabla$-sign) 3 component fit, $(\triangleleft$-sign) 4-component fit. Height of the bars indicates amplitude 
We will now discuss the interpretation of the four-component fit where the second and third components are averaged. The longest relaxation time represents only a small proton population in the sample. This relaxation time is shorter than that of pure water (which is $\sim 2 \mathrm{~s}$ (Ruan, et al., 1999)), but might represent some relatively mobile water within interstices in the protein matrix (Grabowska et al., 2014). The $T_{2}$ value might be lower than that of free water due to pore confinement and by fast proton exchange with dissolved components. The fraction of "free" water is small and negligible above $\sim 20 \mathrm{wt} . \%$ protein, reflecting the strong water binding capacity of SPI. The component with the shortest $T_{2}$ represents protein-bound protons: its amplitude increases linearly with concentration, which is in line with the idea that it represents the proteinbound proton population. The $T_{2}$ of the averaged middle two components represents the vast majority $(>90 \%)$ of the NMR relaxometry signal intensity and is therefore assigned to 'absorbed' water. The $T_{2}$ of this component decreases most of all components as a function of protein concentration, reflecting the decreasing mobility of this fraction with increasing protein concentration. The slight dispersion of relaxation rates of this middle fraction is probably be due to heterogeneity within the individual phases. We infer all information about the system from the weighted average $T_{2}$ of the second and third component, which we consider a fingerprint for the hydration state.

Fig. 2.2 shows the $R_{2}$ relaxation rates $\left(R_{2}=1 / T_{2}\right)$ for absorbed water of SPI and WG as a function of concentration. A dependency of the $R_{2}$ with concentrations was found for both the SPI and WG dispersions. For both SPI and WG, a stretched exponential fit was used to describe these curves (the exponents itself do not have any physical meaning).

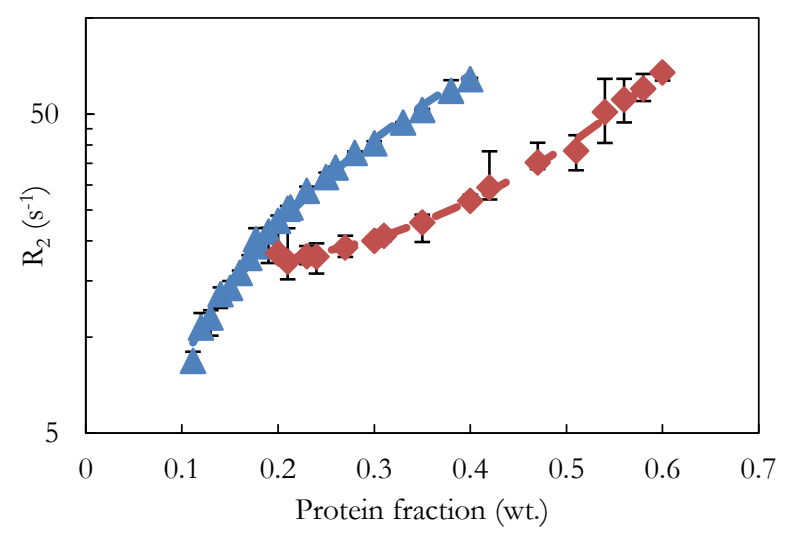

Fig. 2.2 Median $\mathrm{R}_{2}$ of absorbed water for SPI $(\boldsymbol{\Delta})$ and WG $(\diamond)$ plotted with lower and upper bound. Data points were fitted with a stretched exponential: $R_{2, S P I}=1.60 \times 10^{-4} \cdot e^{8.11 \cdot c^{0.127}}(-)$ and $R_{2, W G}=15.9 e^{4.01 \times 10^{-5} \cdot c^{2.57}}(-)$ 


\subsubsection{Prediction of volume fractions with ${ }^{1} \mathrm{H}$ time domain NMR relaxometry}

The $R_{2}$ dependency on concentration for SPI and WG can be used to predict the water distribution in the SPI-WG blends. For a range of possible combinations of weight fractions using mass balances as explained in Appendix $A$, we calculated the weighted average $R_{2}$ values of the corresponding experiments on the separate SPI and WG dispersions. The individual curves were then scaled so that the sum curve represents the concentration of each protein. We assumed no significant diffusive exchange between domains on the timescale of the CPMG experiment $(\sim \mathrm{ms})$, because water molecules (diffusion coefficient $\sim 2 \times 10^{-9} \mathrm{~m}^{2} / \mathrm{s}$ ) would diffuse over a distance of $\sim 2 \mu \mathrm{m}$ on the CPMG time scale, while domains are probably much larger given the low mixing intensity applied during the preparation of the blends.

Fig. 2.3 shows possible combinations of weight fractions for different ratios and SPI-WG blends and separate points that represent the actual measurements on the SPI-WG blends. The points are plotted on the curve where the summed $R_{2}$ values at different concentrations matches the actual $R_{2}$ values. In one case, the $25 \mathrm{wt} . \%$ SPI-WG blend at a ratio of 35:65, the $R_{2}$ value of the blend matches the sum curve of weighted average $R_{2}$ values twice, which means that there are two possible combinations of $R_{2}$ values of SPI and WG that account for the measured value of the blend. The lower SPI-fraction fit is unrealistic, because SPI will necessarily be far more diluted compared to the other four samples with the same ratio SPI and WG. Besides this, WG should be almost completely dry in that blend. 
Ratio 80 : 20

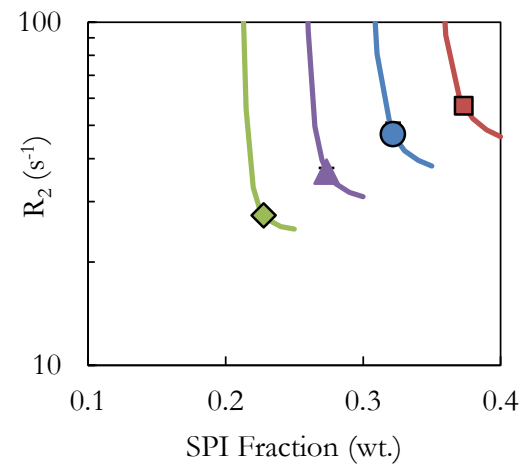

Ratio 50:50

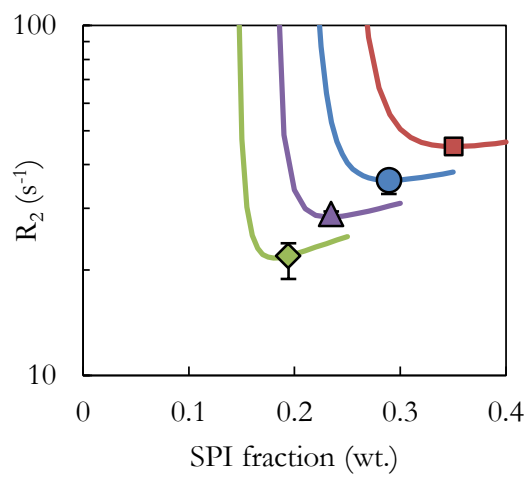

Ratio 20:80

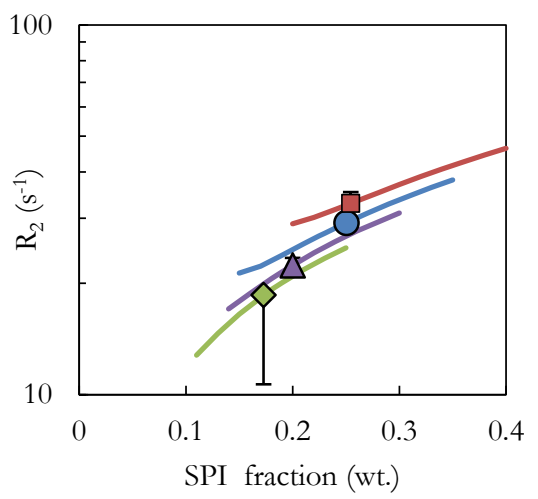

Ratio $65: 35$

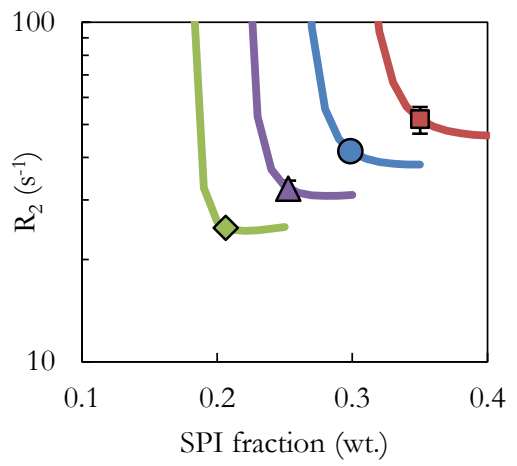

Ratio 35:65

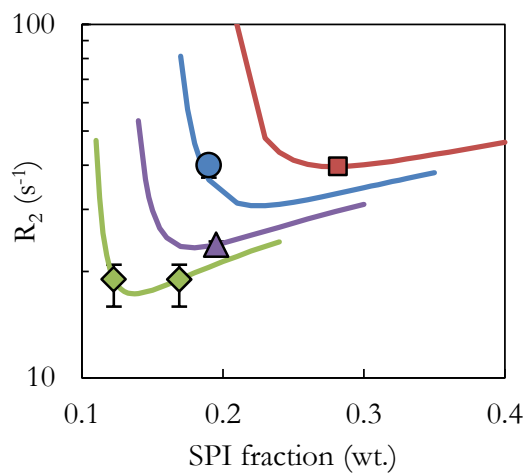

Fig. 2.3 Predicted (curves) and actual (round symbols) median $\mathrm{R}_{2}$ values of absorbed water for SPI - WG blends for different

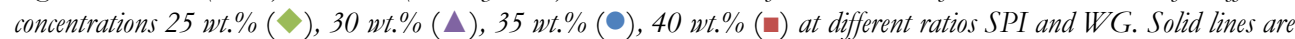
calculated with the stretched exponential curves of SPI and WG 
From the SPI weight fractions, a corresponding SPI volume fraction and WG volume fraction can be calculated using mass balances and expressions for the density (Heldman, Singh, \& Dennis, 2014). Table 2.1 shows that the SPI phase contains relatively more water than the WG phase for all concentrations and all ratios SPI - WG used. Remarkably, the water distribution between the two phases turned out to be mainly dependent on the SPI - WG ratio and rather independent of total dry matter. Obviously, the water binding of SPI is stronger than the water binding of WG also at lower water concentrations.

Table 2.1 Overview results volume fractions determined with TD-NMR and rheology based on isostrain $(n=1)$, bicontinuous $(n=0.2)$ and isostress models $(n=-1)$

\begin{tabular}{|c|c|c|c|c|c|c|c|c|c|}
\hline \multirow[t]{2}{*}{$\begin{array}{l}\text { Ratio } \\
\text { SPI/WG }\end{array}$} & \multirow[t]{2}{*}{$\begin{array}{l}\text { Dry } \\
\text { matter }\end{array}$} & \multicolumn{2}{|c|}{ Volume fraction } & \multicolumn{2}{|c|}{ Volume fraction } & \multicolumn{2}{|c|}{ Volume fraction } & \multicolumn{2}{|c|}{$\begin{array}{l}\text { Volume } \\
\text { fraction }\end{array}$} \\
\hline & & SPI & WG & SPI & WG & SPI & WG & SPI & WG \\
\hline & & \multicolumn{2}{|c|}{ TD-NMR } & \multicolumn{2}{|c|}{$\begin{array}{l}\text { Rheology } \\
(n=1)\end{array}$} & \multicolumn{2}{|c|}{$\begin{array}{l}\text { Rheology } \\
(n=0.2)\end{array}$} & \multicolumn{2}{|c|}{$\begin{array}{l}\text { Rheology } \\
(n=-1)\end{array}$} \\
\hline \multirow[t]{4}{*}{$80 / 20$} & 0.25 & 0.88 & 0.12 & 0.94 & 0.06 & 0.92 & 0.08 & & \\
\hline & 0.30 & 0.88 & 0.12 & 0.95 & 0.05 & & & & \\
\hline & 0.35 & 0.88 & 0.12 & & & 0.85 & 0.15 & 0.87 & 0.13 \\
\hline & 0.40 & 0.86 & 0.14 & & & 0.82 & 0.18 & 0.86 & 0.14 \\
\hline \multirow[t]{4}{*}{$65 / 35$} & 0.25 & 0.78 & 0.22 & 0.87 & 0.13 & 0.87 & 0.13 & 0.80 & 0.20 \\
\hline & 0.30 & 0.78 & 0.22 & 0.89 & 0.11 & & & & \\
\hline & 0.35 & 0.77 & 0.23 & & & 0.73 & 0.27 & 0.77 & 0.23 \\
\hline & 0.40 & 0.71 & 0.29 & 0.74 & 0.26 & 0.77 & 0.23 & 0.78 & 0.22 \\
\hline \multirow[t]{4}{*}{$50 / 50$} & 0.25 & 0.69 & 0.31 & 0.70 & 0.30 & & & 0.71 & 0.29 \\
\hline & 0.30 & 0.66 & 0.34 & & & 0.66 & 0.34 & 0.70 & 0.30 \\
\hline & 0.35 & 0.61 & 0.39 & & & 0.65 & 0.35 & 0.68 & 0.32 \\
\hline & 0.40 & 0.60 & 0.40 & 0.69 & 0.31 & 0.70 & 0.30 & 0.70 & 0.30 \\
\hline \multirow[t]{4}{*}{$35 / 65$} & 0.25 & 0.54 & 0.46 & 0.51 & 0.49 & & & & \\
\hline & 0.30 & 0.57 & 0.43 & & & 0.60 & 0.40 & 0.60 & 0.40 \\
\hline & 0.35 & 0.55 & 0.45 & & & 0.50 & 0.50 & 0.74 & 0.26 \\
\hline & 0.40 & 0.49 & 0.51 & & & & & 0.48 & 0.52 \\
\hline \multirow[t]{4}{*}{$20 / 80$} & 0.25 & 0.30 & 0.70 & & & & & 0.36 & 0.64 \\
\hline & 0.30 & 0.31 & 0.69 & 0.19 & 0.81 & & & 0.38 & 0.62 \\
\hline & 0.35 & 0.29 & 0.71 & & & 0.28 & 0.72 & 0.33 & 0.67 \\
\hline & 0.40 & 0.32 & 0.68 & & & 0.22 & 0.78 & 0.26 & 0.74 \\
\hline
\end{tabular}




\subsubsection{Rheology for SPI and WG dispersions and SPI-WG blends}

Frequency sweep tests were performed for SPI and WG dispersions and SPI-WG blends. Some typical results of a frequency sweep are shown in Fig. 2.4, which depict the measurements of a $40 \mathrm{wt} . \%$ SPI dispersion, a $40 \mathrm{wt} . \%$ WG dispersion and a $40 \mathrm{wt} . \%$ SPI-WG blend at a ratio of 50:50. A slight dependence of the storage modulus on oscillation frequency was found for both SPI and WG dispersion and the blend when plotted on a logarithmic scale. The frequency dependency of SPI with varying oscillation frequency was also found for soybean gels prepared with 25 wt.\% dry matter (Van Kleef, 1986). Kontogiorgos et al. (2007) did not find this dependence for $40 \mathrm{wt} . \%$ gluten, however these samples were aged at $-13^{\circ} \mathrm{C}$, which could have affected the viscoelastic properties (Kontogiorgos, Goff, \& Kasapis, 2007).

The storage and loss modulus were similar for SPI and the SPI-WG blend, whereas the storage and loss modulus for the WG dispersion was clearly lower. Besides, the difference between storage and loss modulus is larger for the SPI and the blend compared to the WG dispersion.

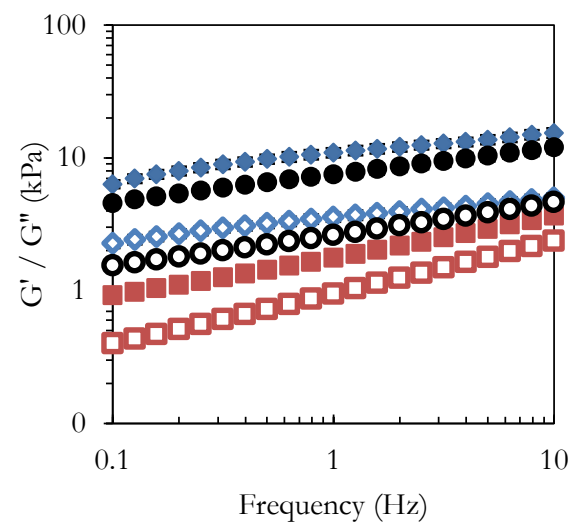

Fig. 2.4 Frequency sweep for $40 \mathrm{wt. \%}$ SPI $(\bullet), 40 \mathrm{wt} . \% \mathrm{WG}(\square)$ and $40 \mathrm{wt} . \%$ SPI-WG blend (ratio 1:1) (•), closed symbols for G' and open symbols for $G$ "

The storage modulus at $1 \mathrm{~Hz}$ was extracted from the frequency sweeps, Fig. 2.5 depicts the storage modulus (at $1 \mathrm{~Hz}$ ) of SPI and WG dispersions and SPI-WG blends as a function of concentration. SPI and WG were mixed at different ratios (80/20, 65/35, 50/50, 35/65 and 20/80). Both SPI and WG dispersions showed a dependency of the storage modulus with concentration, but the storage and loss modulus of the SPI dispersions were higher than that of WG dispersions when comparing the same concentration. The storage modulus as a function of protein concentration were fitted to a power law $G^{\prime}=G_{0} c^{n}$, in which $c$ is the protein weight 
fraction and $G$ 'the storage modulus. A power law relationship between the storage modulus and protein concentration was found in literature for soy protein, but also for other proteins such as ovalbumin, and other plant proteins such as pea protein and fababean (Arntfield, Murray, \& Ismond, 1990; Bikbov, Grinberg, \& Tolstoguzov, 1979; Sun \& Arntfield, 2011; Van Kleef, 1986). At varying concentrations of SPI in water, the concentration dependency was different and two regimes were distinguished. For SPI, the complete concentration range was taken into account to determine $G_{0}^{\prime}$ and $n$; At low concentrations of SPI ( $\left.<20 \mathrm{wt} . \%\right)$, samples were more liquidlike. At these low concentrations, the storage modulus could be fitted with a power law $G^{\prime}=$ $1.88 \times 10^{-4} \cdot c^{5.76}$. The values for the parameters are in the same ranges as reported earlier (Bikbov, et al., 1979) for the SPI concentration range of 10 to $18.5 \mathrm{wt} \%$. At high SPI concentrations (> $20 \mathrm{wt} . \%$ ), samples became more solid-like, leading to a storage modulus that was almost linear and could be fitted with another power law $G^{\prime}=121 \times c^{1.24}$. For WG, the concentration range that resulted in reliable rheological measurements turned out to be between 37.5 wt. $\%$ and 55 wt. $\%$. At lower WG-concentrations (<37.5 wt. \%), water was expelled during the oscillatory tests, whereas high WG-concentrations ( $>55 \mathrm{wt} . \%)$ resulted in a dry and inhomogeneous sample. Research on wheat gluten, for dough applications, often use similar concentration ranges (Bosmans, et al., 2012; Kontogiorgos, et al., 2007). The measurable range was fitted with power law $G^{\prime}=1.74 \times 10^{-2} \cdot c^{3.11}$.

The storage modulus of SPI-WG blends were plotted as separate points in Fig. 2.5. Similarly to the low dry matter samples with WG-concentration, water was expelled during the oscillatory test for SPI-WG blends containing less than $25 \mathrm{wt} \% \%$ and high WG content. That is why 25 wt. $\%$ was taken a minimum concentration for the blend in this analysis. Also for SPI-WG blends, a concentration dependency of the storage modulus was found. It was found that blends with more SPI resembled the behaviour of SPI dispersion and samples that contained more WG resembled WG dispersions. 


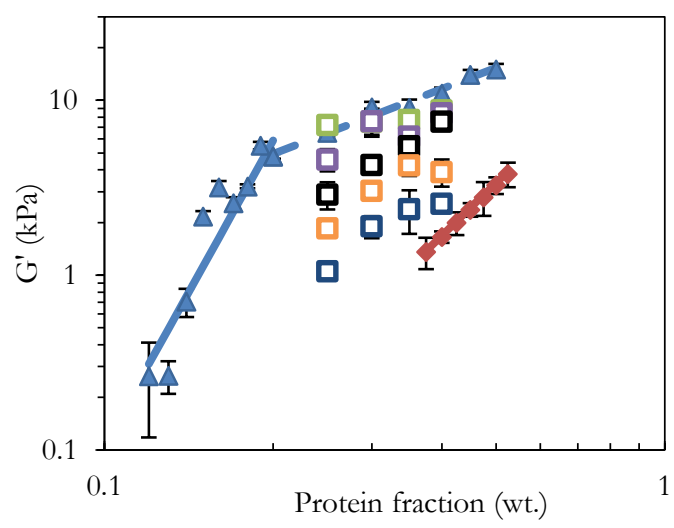

Fig. 2.5 Storage modulus as a function of dry matter for SPI $(\Delta)$ and WG $(\nabla)$ plotted with the standard deviations. Data points were fitted with a power law. SPI was fitted with two power laws: concentration below $20 \mathrm{wt} \% G^{\prime}=1.88 \times 10^{-4}$. $c^{5.76}$ (-) and above $20 \mathrm{wt} \% G^{\prime}=121 \cdot c^{1.24}$ (-). Wheat gluten. $G^{\prime}=1.74 \times 10^{-2} \cdot c^{3.11}(-)$. The SPI-WG

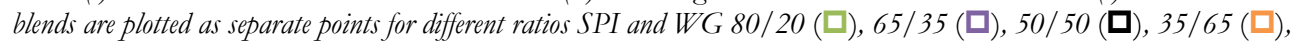
$20 / 80(\square)$

\subsubsection{Estimation of volume fractions with rheology}

The polymer blending law was used for prediction of water distributions in SPI-WG blends. The fitted volume fraction-values are provided in Table 2.1 for the three models. As can be seen, the predicted values for the volume fractions corresponded to the TD-NMR values well. The analysis of the rheological data confirmed that SPI absorbed most of the water in all blends. Unfortunately, it was difficult to discriminate between the three polymer blending law models. To understand this difficulty, we plotted typical results of polymer blending law fits (Fig. 2.6). Here, the curves of isostress, isostrain, and bicontinuous were plotted while a diamond was placed for the actual storage modulus of the blend $\left(G_{c}^{\prime}\right)$ at the corresponding SPI fraction. We noticed that the models predicted a similar value for the $G_{c}^{\prime}$ - values at the conditions measured for most of the blends, allowing no discrimination between the models. The main reason for this behaviour is that water distribution influences both the moduli of the phase as well as the volume fractions in our simulations of the rheological properties.

Nevertheless, a number of conclusions can be drawn about the microstructure. Given the water contents in both phases, it can be stated that the storage modulus of the SPI was larger than storage modulus of the WG phase in all cases when using the power law fits presented above and the water contents given by TD-NMR. The higher value of SPI was remarkable given the higher water content in that phase. For the blends having a SPI-WG ratio of $80 / 20$, it turned out that SPI had even a much higher loss modulus than the WG-phase. Given the high volume 
fraction of this blend, it can be expected that SPI will form the continuous phase and WG the dispersed phase, which presence will lead to a slight weakening of the blend compared to the strength of the SPI-phase. For SPI-WG blends with ratio 20:80, WG becomes the continuous phase and SPI the dispersed phase. Because the storage modulus of SPI is higher than the storage modulus of WG in that blend for all concentrations, it can be expected that SPI will act as hard filler particles in a continuous WG phase. The situation for the other blends is less clear. For the blends having a ratio of SPI-WG ratio of $35 / 65,50 / 50$ and $65 / 35$, the storage modulus of SPI was on average twice the value of the storage modulus for WG. This means that a clear discrimination of the exact structure and related polymer blending law becomes difficult. Nevertheless, the exact volume fractions can be quantified well, giving new insights in the behaviour of the blends. The actual processing will define the final structure of the blend as further explored in a previous study (Grabowska, Tekidou, Boom, \& van der Goot, 2014). When comparing that study with the results in this paper, it seems that the rheological properties of both phases should be similar for optimal alignment of the material using simple shear flow. This makes this study relevant as it provides a tool that can be used to predict the structure formation in those dense biopolymer blends. 
30 wt. \% SPI-WG - Ratio $80: 20$

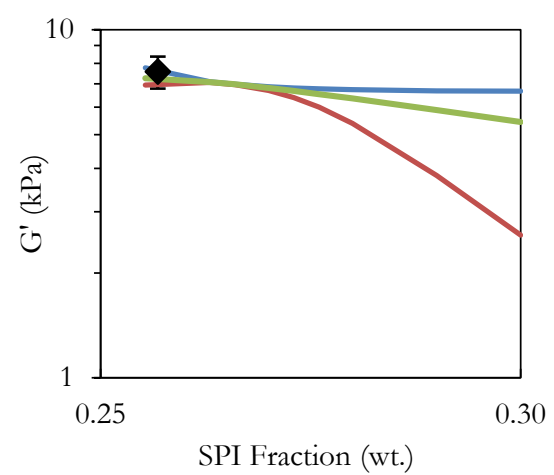

30 wt. \% SPI-WG - Ratio 50:50

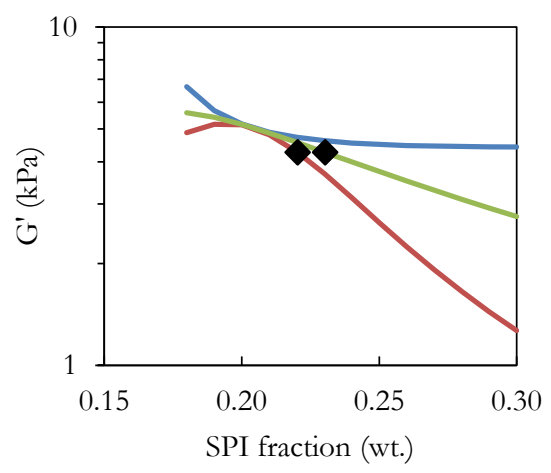

40 wt. \% SPI-WG - Ratio 20:80

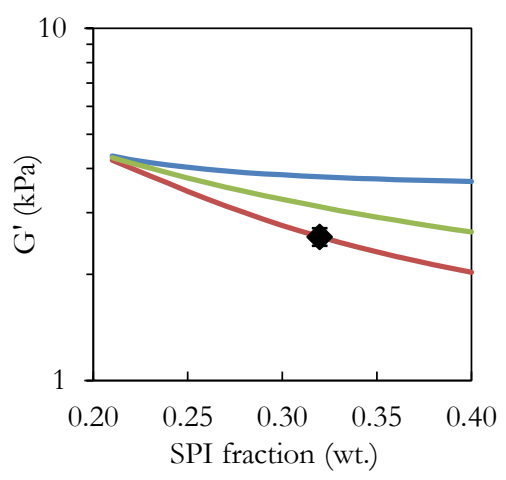

40 wt.\% SPI-WG - Ratio $65: 35$

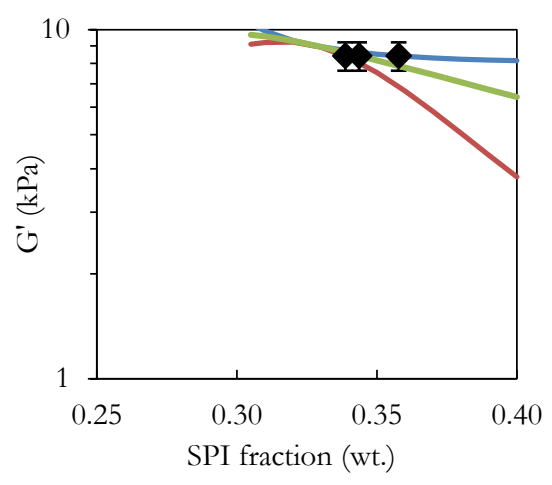

40 wt. \% SPI-WG - Ratio 35:65

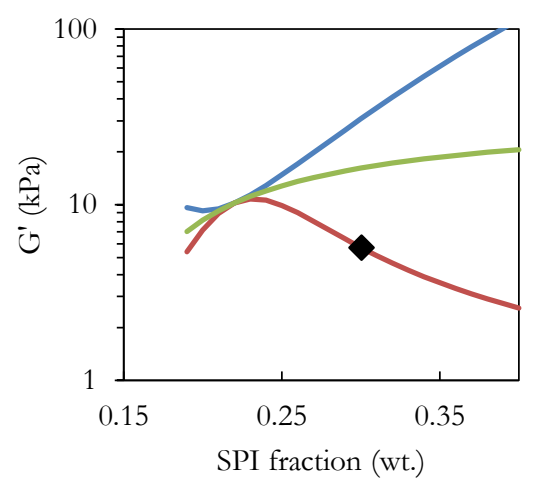

Fig. 2.6 Polymer blending law applied for isostrain (-), isostress (-) and bicontinuous (-) models, and $G_{C}^{\prime}(-)$ for SPI$W G$ blends at different ratios of SPI and WG 


\subsection{Conclusions}

To better understand the rheological properties of biopolymer blends, it is important to be able to determine water distribution with an independent measurement. So far, rheological data were interpreted using water distribution as fit parameter. We successfully developed a method based on TD-NMR that allowed a direct and accurate measure of water distribution in a biopolymer blend comprising soy protein isolate (SPI) and wheat gluten (WG).

Water distribution between the two phases as obtained from TD-NMR was compared to estimations of the same water distribution obtained from rheological measurements using the polymer blending law. Both approaches showed that the SPI-phase clearly contained more water than the WG-phase. The quantitative agreement between both methods was remarkably good. Rheology indicated that the SPI phase did not only contain most water, but also had the highest storage modulus.

We compared the outcomes to a previous study in which the same blend was used for making anisotropic structures. We found a good relation between matching rheological properties and structuring behaviour. We therefore conclude that the approach presented in this paper is not only useful to better understand water distribution and resulting rheological properties in a dense biopolymer blend, it also can help to define the composition, which is most suitable for creating anisotropic biopolymer blends.

\section{Acknowledgements}

The authors thank Frank Vergeldt for valuable discussions and technical support. Ayesha Sarker and Bas Ooteman are acknowledged for contribution to the experimental results. We acknowledge Barentz, NL for materials. We thank the Institute for Sustainable Process Technology (ISPT) for collaboration. This research received funding from The Peas Foundation and the Netherlands Organization for Scientific Research (NWO) in the framework of the Technology Area COAST. 


\section{Appendix}

\section{Appendix A. Calculation of water content in SPI-WG blend.}

The following blend (Fig. A.1) consisting of SPI with water (phase 1) and WG with water (phase 2 ) is considered. We assume complete demixing (i.e. no SPI in the WG phase and vice versa).

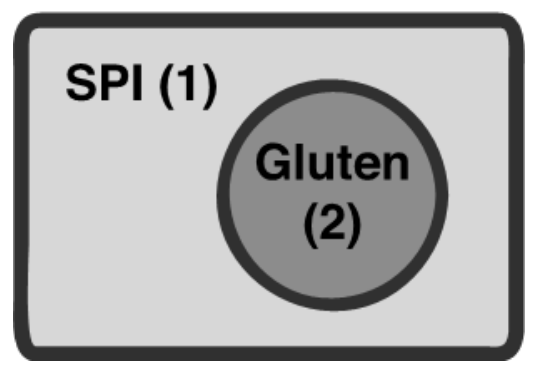

Fig. A.1 Schematic picture of the SPI-WG blend

The following symbols are defined: $W_{S P I}$ is the amount of SPI added to the blend $(\mathrm{kg}), W_{W G}$ is the amount of WG added to the blend $(\mathrm{kg}), W_{w}$ is the amount of water added to the blend $(\mathrm{kg})$

$$
C_{S P I, o v}=\frac{W_{S P I}}{W_{S P I}+W_{W G}+W_{w}}
$$

is the concentration of SPI in the overall blend $(\mathrm{kg} / \mathrm{kg})$

$$
C_{W G, o v}=\frac{W_{g}}{W_{S P I}+W_{W G}+W_{w}}
$$

is the concentration of WG in the overall blend $(\mathrm{kg} / \mathrm{kg})$

Since no SPI will be present in phase 2, all SPI added with end up in phase 1:

$$
C_{S P I, 1}==\frac{W_{S P I}}{W_{S P I}+W_{w, 1}}
$$

is the concentration of SPI in phase $1(\mathrm{~kg} / \mathrm{kg})$.

Since WG will be present in phase 1, all WG added will end up in phase 2:

$$
C_{W G, 2}=\frac{W_{W G}}{W_{W G}+W_{w, 2}}
$$

is the concentration of WG in phase $2(\mathrm{~kg} / \mathrm{kg})$. 
The volume fraction of phase 1 reads

$$
\phi_{1}=\frac{\left(w_{S P I}+w_{w, 1}\right) / \rho_{1}}{\left(w_{S P I}+w_{W G}+w_{w}\right) / \rho_{o v}}
$$

The volume fraction of phase 2 reads

$$
\phi_{2}=\frac{\left(w_{W G}+w_{w, 2}\right) / \rho_{2}}{\left(w_{S P I}+w_{W G}+w_{w}\right) / \rho_{o v}}
$$

with $\rho_{1}\left(\mathrm{~kg} / \mathrm{m}^{3}\right)$ being the density of the SPI phase, $\rho_{2}\left(\mathrm{~kg} / \mathrm{m}^{3}\right)$ the density of the WG phase and $\rho_{o v}\left(\mathrm{~kg} / \mathrm{m}^{3}\right)$ the density of the total blend.

The relation between the concentration and the volume fraction can now be calculated. To do so, we have to find an equation for $w_{w, 1}$. This needs rewriting of equation (A1.3):

$$
w_{w, 1}=\frac{w_{S P I}}{C_{S P I, 1}}-w_{S P I}
$$

which equation can then be combined with (A1.5) leading to

$$
\phi_{1}=\frac{w_{S P I} / \rho_{1}}{C_{S P I, 1}\left(w_{S P I}+w_{W G}+w_{w}\right) / \rho_{o v}}
$$

a similar equation can be derived for phase 2 . 


\section{Appendix B. Time domain NMR relaxometry: Amplitudes and T2's of four-component fit as a function of SPI concentration.}

Plots of the residual sum of squares (RSS, the sum of squared differences between fit and data) as a function of the number of components consistently show that fitting a 2nd, 3rd and 4th component leads to a tangible reduction of the differences, whereas inclusion of a 5 th does not. Fig. B.1 shows those plots for $20 \mathrm{wt.} \% \mathrm{WG}$ and $20 \mathrm{wt} . \%$ SPI dispersions as an example.

A

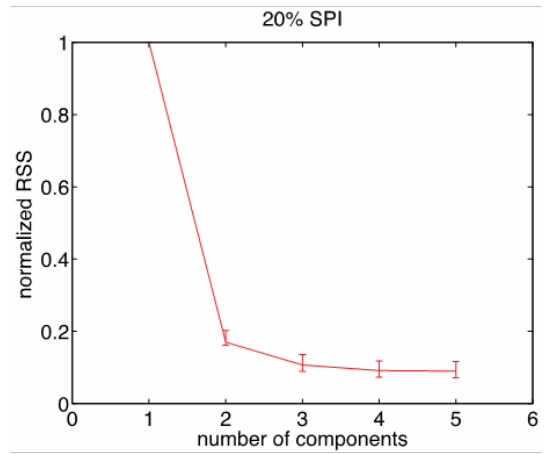

B

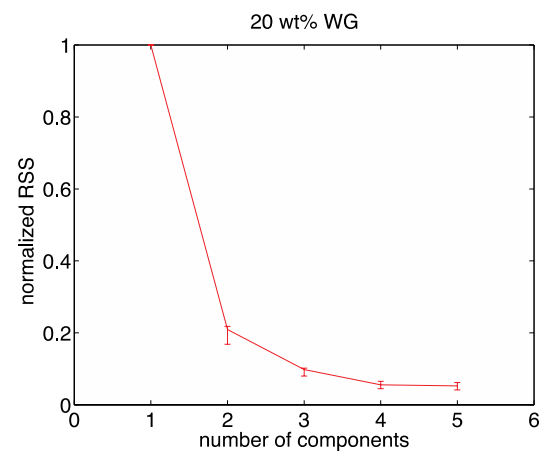

Fig. B.1. RSS dependence of the number of fitted components in two samples: A) $20 \mathrm{wt} . \%$ SPI, B) $20 \mathrm{wt} \% \mathrm{WG}$. For the 1-component fits, RSS is normalized to 1. The error bars are obtained from $>5$ experimental replicates. 
Chapter 2 


\section{Chapter 3}

The phase properties of soy protein and wheat gluten in a blend for fibrous structure formation

This chapter has been published as B.L. Dekkers, M.A. Emin, R.M. Boom, \& A.J. van der Goot, (2018). The phase properties of soy protein and wheat gluten in a blend for fibrous structure formation. Food Hydrocolloids, 79, 273-281. http://doi.org/10.1016/j.foodhyd.2017.12.033 


\section{Abstract}

Fibrous macrostructures can be obtained from among others soy protein isolate (SPI) - wheat gluten (WG) blends, when deformed with simple shear flow while heating. This SPI-WG blend consists of two phases, which are separated on a micrometre scale. The properties, especially the rheological properties, of SPI- and WG-phases determine the structure formation process largely. The objective of this study was to determine the properties of the phases present in SPI - WG blends using conditions relevant for fibrous structure formation. Since, the rheological properties are dependent on the concentration of each protein in its respective phase, we determined the water content in the two phases with a methodology based on time-domain nuclear magnetic resonance. The spatial distribution of the two phases was studied with confocal scanning laser microscopy, and the rheological properties were measured with three types of oscillatory rheology. The experiments showed that the SPI-phase absorbed more water than the WG-phase, which resulted in a larger volume fraction than mass fraction of the SPI-phase and vice versa for WG. The absorption of more water by SPI resulted in a lower concentration of SPI in that phase, remarkably leading to a $G^{\prime}$-value for SPI that was similar to the rheological properties of WG. Similarities in rheological properties seem to be required to strongly deform and align the dispersed phase and form fibrous macrostructures. 


\subsection{Introduction}

Industrial meat production negatively impacts the environment in terms of climate change, land use, water use, greenhouse gas emission and energy consumption (Steinfeld, Gerber, Wassenaar, Castel, \& de Haan, 2006). Plant-based meat analogues may offer a more sustainable alternative. Consumer research showed that one should aim either for products that resemble meat, or should be clearly different from meat and have their own identity (Elzerman, Boekel, \& Luning, 2013; Elzerman, Hoek, van Boekel, \& Luning, 2015; Hoek et al., 2011). To produce meat analogues that resemble meat, a technology based on simple shear-flow deformation was introduced a decade ago (Manski et al., 2007b). This technology was used to obtain anisotropic, fibrous macrostructures through shearing while heating concentrated plant protein blends, such as soy protein concentrate, soy protein isolate combined with pectin, and soy protein isolate (SPI) combined with wheat gluten (WG) in a high temperature shear cell (Dekkers, Nikiforidis, \& Goot, 2016; Grabowska et al., 2016; Grabowska, Tekidou, Boom, \& van der Goot, 2014; Krintiras, Gadea Diaz, van der Goot, Stankiewicz, \& Stefanidis, 2016; Krintiras, Göbel, Bouwman, van der Goot, \& Stefanidis, 2014; Krintiras, Göbel, van der Goot, \& Stefanidis, 2015). It was concluded that at least a two-phase system is needed to produce anisotropic, fibrous macrostructures, since SPI alone resulted in layered structures only. The fibrous structures become visual upon tearing the material, and originates from detachment through or along a weak, deformed dispersed phase (Dekkers, Hamoen, Boom, \& van der Goot, 2018; Dekkers, Nikiforidis, \& Goot, 2016).

The creation of fibrous macrostructures from SPI-WG blends was reported to require a careful balance between the blend properties and process conditions (Grabowska et al., 2014). The composition including overall dry matter content and volumetric ratio between the components determined the morphology of the structures formed. In addition, the process conditions such as temperature, deformation rate and processing time influenced structure formation (Krintiras et al., 2016, 2014). The composition of SPI-WG blends could be used to create various types of macrostructures such as: isotropic gels, gels with a layered and/or fibrous structures, when deformed with a rotational speed of $30 \mathrm{rpm}$ while heating at $95^{\circ} \mathrm{C}$ (Grabowska et al., 2014). A fibrous macrostructure is formed by simultaneous deformation and alignment on a microstructural scale of the SPI- and WG-phase in the shear flow, which requires similarities in rheological properties of the phases. The deformability of the two phases is among others related 
to the relative proportions of the two components (phase volumes), their spatial distribution and the rheological properties; all depend on the water distribution between the two phases.

Rheological properties of blended materials have been extensively studied with the polymer blending law (Clark, Richardson, Ross-Murphy, \& Stubbs, 1983; Kasapis \& Al-Marhoobi, 2005; Kasapis \& Tay, 2009; Morris, 1992, 2009). The polymer blending law is an empirical law that was established by Takayanagi (Takayanagi, Harima, \& Iwata, 1963), and reads in its general form as:

$$
G_{X Y}^{\prime}=\Phi_{X} \cdot\left(G_{X}^{\prime}\right)^{n}+\Phi_{Y} \cdot\left(G_{Y}^{\prime}\right)^{n}
$$

in which $G_{X}^{\prime}, G_{Y}^{\prime}$ and $G_{X Y}^{\prime}$ are the moduli of phase X, phase $\mathrm{Y}$ and the blended material $(\mathrm{XY})$, $\Phi_{X}$ and $\Phi_{Y}$ are the volume fractions of phase $\mathrm{X}$ and $\mathrm{Y}$. The value of the parameter $n$ depends on the spatial distribution of the two components in the blended material and their moduli. For phase separated blends in which the dispersed phase is softer compared with the continuous phase, an isostrain model is used with $n=1$. For blends with rigid dispersed particles in a weaker continuous phase, the isostress model is generally used with $n=-1$. Since the volume fractions of the two phases are mostly unknown, a fitting parameter, referred to as the avidity parameter/p-value, is often used (Clark et al., 1983; Fitzsimons, Mulvihill, \& Morris, 2008; Morris, 1992). Furthermore, complementary use of rheology and imaging was shown to be an useful tool for understanding to which extent microstructure and mechanical properties are related (Firoozmand, Murray, \& Dickinson, 2009; Kasapis \& Tay, 2009).

Water distribution in a phase separated system can also be directly determined with time-domain nuclear magnetic resonance (TD-NMR) (Clark et al., 1983). A methodology based on TD-NMR was developed in our previous study to determine the amount of water absorbed by SPI and WG in this concentrated blend after mixing at room temperature (Dekkers et al., 2016). It was assumed that these two proteins are completely phase separated and relatively large in size. With TD-NMR, the proton transverse relaxation rate $\left(R_{2}\right)$ of water was determined at various protein concentrations of SPI and WG, separately and in the blend. The $R_{2}$-values were used as input to obtain estimates for the water distribution (and phase volume fractions) in the SPI-WG blends.

In this study, TD-NMR, confocal scanning laser microscopy (CSLM) and rheology were combined to determine the properties of phases present in concentrated SPI-WG blends to understand their deformability at elevated temperatures. Since creation of fibrous structures is a careful balance between the blend properties and the process conditions, these blend properties 
should be measured under similar conditions as used during actual processing of the blend. The objective of this study was therefore to determine the properties of a concentrated SPI-WG blend and the phases present in the blend during and after heating and shearing. Insights in the blend properties under these conditions can be used to better understand deformation and alignment of this blend during simple shear flow deformation (Grabowska et al., 2014; Krintiras et al., 2016, 2014, 2015). First, TD-NMR was used to determine how water distributed between SPI and WG after heating (and shearing). A mass balance was then used to calculate the phase volumes of SPI and WG. With confocal scanning laser microscopy (CSLM), we studied the spatial distribution and microstructure of heated SPI - WG blends. The phase volume was used as input for the polymer blending law to determine the rheological properties of the SPI and WG-phase in the blended material. The rheological properties of SPI, WG and SPI-WG were measured under shear while heating in a closed cavity rheometer (CCR). Furthermore, the rheological properties of SPI, WG and SPI-WG were measured on gels after heating and subsequent cooling to room temperature. The composition of the blend was varied in dry matter content and ratio between SPI and WG. The differences in the blend properties were related to the ability of the various blends to form fibrous structures in a high temperature shear cell.

\subsection{Materials and methods}

\subsubsection{Materials}

For sample preparation, soy protein isolate (SPI) and wheat gluten (WG) were used, both obtained from Barentz (Hoofddorp, The Netherlands). SPI (SUPRO®, EX 37) contains $>90$ wt. $\%$ protein, $<6$ wt. $\%$ moisture, $<5$ wt. $\%$ ash and $<1$ wt. $\%$ free fat as defined by the manufacturer (Solae, St. Louis, Missouri, USA. WG (Vital Wheat) has protein content of $>83$ wt. $\%$ and 8 wt. $\%$ moisture as defined by the manufacturer (Roquette, Lesterm, France). Foodgrade $\mathrm{NaCl}$ was obtained from Sigma-Aldrich (Zwijndrecht, The Netherlands). 


\subsubsection{Methods}

In Table 3.1, an overview is shown of the various samples that were used per analytical method.

Table 3.1 Overview experimental design and sample treatments ( ${ }^{*}=$ SPI:WG: 20:80, 35:65, 50:50, 65:35, 80:20)

\begin{tabular}{|c|c|c|}
\hline Analysis & Gelled samples & Sheared samples \\
\hline TD-NMR & $17.5-45$ wt. $\%$ SPI & - \\
\hline \multirow[t]{3}{*}{$(\mathrm{n}=4)$} & $35-50$ wt. $\%$ WG & - \\
\hline & 25 - 40 wt. $\%$ SPI-WG & $27.5,30$, and 35 wt. $\%$ \\
\hline & & SPI-WG \\
\hline \multirow[t]{3}{*}{$\overline{\text { CSLM }}$} & $30 \mathrm{wt} . \%$ SPI & \\
\hline & 37.5 wt. $\%$ WG & - \\
\hline & $30 \mathrm{wt} . \%$ SPI-WG & \\
\hline Rheology with CCR & & $17.5-45$ wt. $\%$ SPI \\
\hline \multirow[t]{2}{*}{$(\mathrm{n}=2)$} & - & $35-50$ wt. $\%$ WG \\
\hline & & 25 - 40 wt. $\%$ SPI-WG \\
\hline Rheology with & $17.5-45$ wt. $\%$ SPI & \\
\hline stress-controlled rheometer & $35-50$ wt. $\%$ WG & - \\
\hline$(\mathrm{n}=4)$ & 25 - 40 wt. $\%$ SPI-WG & \\
\hline
\end{tabular}

\subsubsection{Preparation of samples in a high temperature shear cell}

The structuring process was adapted from Grabowska, Tekidou, Boom, \& van der Goot, 2014. In short, SPI was dispersed in a $\mathrm{NaCl}$ solution (1 wt.\%) by thorough mixing with a spatula, and hydrated for $30 \mathrm{~min}$. The pre-moisturized SPI was combined and mixed with WG in a ratio of 50:50 with a final protein content of 27.5, 30 and $35 \mathrm{wt} . \%$. The protein blends were put in a preheated shear cell of $95^{\circ} \mathrm{C}$ and sheared for $15 \mathrm{~min}$ at $30 \mathrm{rpm}$. After shear-induced structuring, the samples were cooled to room temperature in $5 \mathrm{~min}$. The shear cell samples were stored overnight before doing TD-NMR.

\subsubsection{Preparation of protein dispersions and gels}

Aqueous dispersions of SPI, WG, and SPI-WG blends were prepared using double distilled water containing 1 wt. $\% \mathrm{NaCl}$. For the SPI-WG blends, different ratios of the proteins were mixed $(20 / 80,35 / 65,50 / 50,65 / 35$, and $80 / 20)$. The protein powder was added to the salt solution. The dispersions were thoroughly mixed using a spatula. Even at the lowest protein contents used, the dispersions were sufficiently viscous to prevent settling. All dispersions were allowed to equilibrate at room temperature for $30 \mathrm{~min}$ before they were either sheared while 
heated in the CCR (sheared samples) or were heated to prepare the gelled samples. For a few samples, the SPI was also hydrated for 30 min before the WG was added, similar to preparation of sheared samples. No differences were found between these two preparation methods when analysed in terms of water distribution or rheology. To make the gels, samples were placed in air-tight cylindrical wells with a diameter of $25 \mathrm{~mm}$ and height of $4 \mathrm{~mm}$. To remove most of the air from the sample material, the cylindrical wells were overfilled. The surplus of the hydrated proteins was allowed to escape via the hole on top of the well. Then this hole was sealed before heating. The gels were then obtained by heating at $95^{\circ} \mathrm{C}$ for $15 \mathrm{~min}$ in a water bath, followed by cooling to room temperature through placing the well in a cold water bath over $5 \mathrm{~min}$. The gels were stored overnight before carrying out TD-NMR or rheology.

\subsubsection{Time domain nuclear magnetic resonance (TD-NMR)}

The method for TD-NMR was described in a previous paper (Dekkers et al., 2016). Samples were transferred into $7-\mathrm{mm}$ NMR tubes. ${ }^{1} \mathrm{H}$ relaxometry was performed at $0.72 \mathrm{~T}$ magnetic field strength (30.7 MHz ${ }^{1} \mathrm{H}$ resonance frequency) with a Maran Ultra NMR spectrometer. The spectrometer was controlled by RINMR software (Resonance Instruments Ltd., Witney, UK). During a Carr-Purcell-Meiboom-Gill (CPMG) experiment, five data points per echo and in total 12288 echoes were recorded, and the time between each echo was $407 \mu \mathrm{s}$. The sample time between the data points in each echo was $10 \mu$ s resulting in a spectral width of $100 \mathrm{kHz}$. In total, 16 transients were recorded with phase cycling resulting in no baseline offset, with a repetition time of $30 \mathrm{~s}$. Each echo was averaged to one data point, and each CPMG echo train was phase corrected using the IDL package (ITT Visual Information Solutions, Boulder, CO, USA). The experimental error (upper and lower bound) was measured by repeating all experiments four times.

The Levenberg-Marquardt non-linear least squares algorithm implemented in SplMod (Vogel, 1988), was used to analyse the decay curves of the transverse magnetization, which fits a sum of exponential curves (“components") $\sum_{\mathrm{i}} \mathrm{A}_{\mathrm{i}} \mathrm{e}^{-\mathrm{t} / T_{2, \mathrm{i}}}$ to the decay, resulting in an amplitude $A$ and transverse relaxation time $T_{2}$ for each component $i$. The inverse of $T_{2}$ is the relaxation rate $R_{2}$. The number of decay components needed to describe the data was estimated from the decrease of the standard deviation when fitting an additional component. For all experiments, we found that the data could be accurately described with four components. Laplace inversion of the data (by CONTIN) predicts three separate $T_{2}$ distributions (Provencher, 1982), similarly as described in our previous study (Fig. 2.1, Chapter 2 (Dekkers et al., 2016)). The two middle components 
(second and third) in a 4-component fit were both used to quantify the large population with intermediate water mobility. The first component, the water population with low mobility, and the fourth component, the water population with high mobility, were not considered further. The second and third components were averaged, which represents the vast majority (>90\%) of the NMR relaxometry signal intensity and was therefore assigned to "absorbed" water. The concentration $\left(c_{i}\right)$ dependency of $\mathrm{R}_{2}$ of "absorbed" water was fitted with a stretched exponential

in which $A, B$ and $C$ were fitting parameters.

$$
R_{2}=A * e^{B * C_{i}{ }^{C}}
$$

\subsubsection{Confocal scanning laser microscopy}

The microstructures of the gels were visualized with a confocal scanning laser microscope. The gels were rapidly frozen with liquid nitrogen. The frozen samples were placed into a cryomicrotome (Micron CR50-H, ADAMAS-instruments, Rhenen, The Netherlands) at a temperature of $-20^{\circ} \mathrm{C}$. The cryo-microtome was used to make regularly shaped slices of approximately $40 \mu \mathrm{m}$. A concentration of $0.2 \mathrm{mg} / \mathrm{ml}$ rhodamine B was used to stain the proteins (Sigma, Steinheim, Germany). Samples were visualized with Confocal Scanning Laser Microscope type 510 (Zeiss, Oberkochen, Germany) using a $543 \mathrm{~nm}$ HeNe laser and a $405 \mathrm{~nm}$ Blue/Violet diode laser. A 20x EC Plan-Neofluar/0.5. A lens was chosen to take the images. In addition we used the bright field channel. The images were analysed with the blue edition of the ZEN software (Carl Zeiss Microscopy).

\subsubsection{Rheology}

Two rheometers were used: i) closed cavity rheometer (CCR) (RPA elite, TA instruments, New Castle, Delaware, USA), and ii) stress-controlled rheometer (MCR 502, Anton Paar, Graz, Austria). The CCR was used to determine the rheological properties under shear and elevated temperatures (Emin, Quevedo, Wilhelm, \& Karbstein, 2017). The protein dispersions were placed in the cavity (disk geometry) of the rheometer, which was sealed to allow a pressure of $4500 \mathrm{kPa}$ to prevent water evaporation. A high strain $(80 \%)$ and frequency $(10 \mathrm{~Hz})$ and high temperature were applied for $15 \mathrm{~min}$ (measurement recorded every second). These measurements were done outside the linear viscoelastic regime (LVR); the stress response is no longer a single-harmonic sinusoid and therefore we refer to an apparent $G$ '. Subsequently, we performed a frequency sweep $(10-0.1 \mathrm{~Hz})$ at a lower strain $(1 \%)$ after the time-sweep experiment (at $95^{\circ} \mathrm{C}$ ) with 9 measurements points. In addition, we also determined the viscoelastic properties of protein gels with the stress-controlled. A serrated parallel plate-plate 
geometry (pp25/p2, Anton Paar) was used to avoid slip. The normal force was set to $0.3 \mathrm{~N}$ with a hysteresis of $0.075 \mathrm{~N}$, which turned out to be sufficient to prevent slip in the linear rheological regime and not too high to deform the sample. These measurements were performed at $25{ }^{\circ} \mathrm{C}$. A strain sweep $(0.5-50 \%, 1 \mathrm{~Hz})$ was performed to determine the viscoelastic region, and a frequency sweep $(10-0.1 \mathrm{~Hz}, 0.5 \%)$ was performed with 21 measurement points.

The apparent $G$ ' is extracted from the time sweep experiment after $15 \mathrm{~min}$. From the frequency sweep measurements, the storage modulus $\left(G^{\prime}\right)$ was extracted at $1 \mathrm{~Hz}$. The $G^{\prime}$ as a function of the protein concentration was fitted to a power law

$$
G^{\prime}=G_{0} c^{N}
$$

in which $c$ is the protein weight fraction, $G^{\prime}$ the storage modulus and $G_{0}$ and $N$ were fitting parameters.

\subsection{Results and discussion}

At sufficiently high dry matter contents, blends of soy protein isolate (SPI) and wheat gluten (WG) are phase separated and form fibrous structures when shear is applied (Grabowska et al., 2014; Krintiras et al., 2016, 2014, 2015). For this reason, we determined the phase volumes of SPI and WG, their spatial distribution and rheological properties in the blend when heated and/or sheared. First, the phase volumes were estimated by determining the water distribution with a methodology based on time-domain nuclear magnetic resonance (TD-NMR). The spatial distribution of SPI and WG was studied with confocal scanning laser microscopy (CSLM). The insights in phase volume and spatial distribution were used to predict the rheological properties of the two proteins in a blend during heating and shearing (sheared samples), and after heating and subsequent cooling (gelled samples).

\subsubsection{Water distribution with TD-NMR}

With TD-NMR, the relaxation rate $\left(R_{2}\right)$ of absorbed water was determined for various types of samples: $i$ ) protein gels, ii) sheared samples prepared in the high temperature shear cell, and iii) untreated protein dispersions (non-sheared and non-heated, data of the latter system were taken from Dekkers et al., 2016). The results are plotted in Fig. 3.1A. There is a clear dependence of the absorbed water $\left(R_{2}\right.$-value from TD-NMR) with protein concentration. However, there was hardly any difference in $R_{2}$-value for untreated protein dispersions, protein gels or fibrous 
samples prepared in the high temperature shear cell. Apparently, these treatments did not influence the $R_{2}$-values.

Fig. 3.1B shows an overview of the $R_{2}$ values for SPI, WG and SPI-WG gels as function of protein concentration. The $R_{2}$-values for SPI were always larger than those for WG at similar protein concentration suggesting that more water was associated to SPI than WG. The $R_{2}$-value for SPI-WG blends were in between the two single component $R_{2}$ values. SPI-WG blends high in SPI (i.e. 80/20 and 65/35) were more similar to SPI, whereas blends high in WG (i.e. 20/80 and 35/65) were more similar to WG. Stretched exponentials were used to fit the relaxation rate for SPI and WG gels as a function of protein concentration. In our previous study, we also used stretched exponentials to fit the $R_{2}$ of (unheated) protein dispersions consisting of SPI and WG, and these curves are shown in Fig. 3.1 as well (dashed line). There were some small differences between the $R_{2}$-values for SPI and WG protein i) gels and ii) dispersions separately. The difference between the stretched exponentials of SPI gels and dispersions suggests that heating resulted in increased water binding to the proteins. At low protein concentrations, the $R_{2}$ value for WG was lower in gels than dispersions, which indicates that the mobility of water is somewhat higher after heating; though differences were small. Bosmans et al. also studied gluten (53 wt. $\%$ in water) after heating $\left(10 \mathrm{~min}\right.$ at $\left.110^{\circ} \mathrm{C}\right)$ and concluded that there were no pronounced changes in the proton distributions associated with the gluten under these conditions (Bosmans et al., 2012).
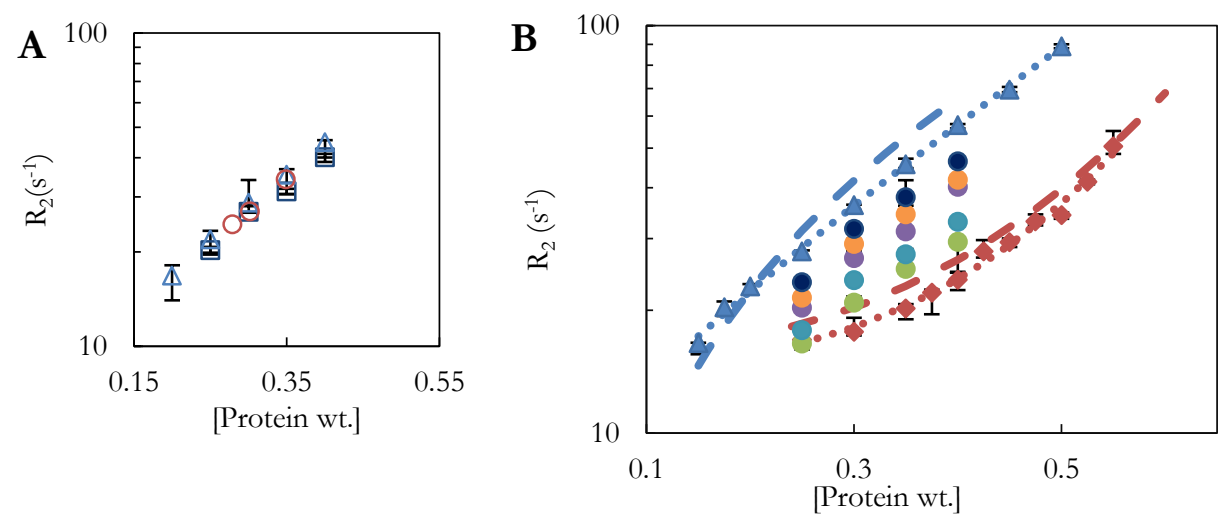

Fig. 3.1 Relaxation rate $\left(\mathrm{R}_{2}\right)$ plotted with lower and upper bound of A) 50/50 SPI-WG protein dispersion $(\Delta)$, gel $(\square)$, and sheared sample $(\bigcirc)$, and B) SPI $(\mathbf{\Delta})$ and $W G(\diamond)$, and SPI-WG gels in various ratios: 20/80 (○), 35/65 (-), 50/50 (-),65/35 (-) and 80/20 (-) plotted with lower and upper bound, and fitted stretched to the exponential

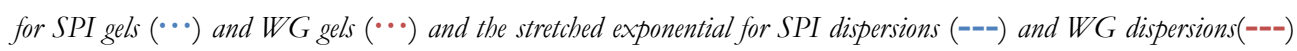
adopted from (Dekekers et al., 2016). 
A mass balance was used to calculate all theoretical possibilities of water distribution between the two phases after gelation. The $R_{2}$ value of the two separate phases at the various theoretical water distributions in the blend were summed (concentration based average) and compared to the experimentally determined $R_{2}$ value of the particular SPI-WG blend after heating. The water distribution was taken at the point where the summation of the experimentally determined $R_{2-}$ values of the blend corresponded to the calculated $R_{2}$ value of the blend. This was possible under the following assumptions: i) SPI and WG are immiscible at high concentrations and form separate phases with only SPI or WG being present in that phase, and ii) diffusive exchange was

Table 3.2 Overview of estimated concentration of SPI in phase 1 and concentration of WG in phase 2 and volume fractions of the SPI and WG-phases determined using time domain-NMR. * = minimum of summed $\mathrm{R}_{2}$-value.

\begin{tabular}{|c|c|c|c|c|c|c|c|c|}
\hline \multirow[t]{2}{*}{$\begin{array}{l}\text { Ratio } \\
\text { SPI/WG }\end{array}$} & $\begin{array}{l}\text { Dry } \\
\text { matter }\end{array}$ & $\begin{array}{l}\mathrm{R}_{2} \\
\text { SPI-WG }\end{array}$ & {$[\mathrm{SPI}]$} & [WG] & $\begin{array}{l}\mathrm{R}_{2} \\
\mathrm{SPI}\end{array}$ & $\begin{array}{l}\mathrm{R}_{2} \\
\text { WG }\end{array}$ & $\Phi_{\mathrm{SPI}}$ & $\Phi_{\mathrm{WG}}$ \\
\hline & $(w t)$ & $\left(\mathrm{s}^{-1}\right)$ & $(\mathrm{g} / \mathrm{g})$ & $(\mathrm{g} / \mathrm{g})$ & $\left(\mathrm{s}^{-1}\right)$ & & $(-)$ & $(-)$ \\
\hline \multirow[t]{4}{*}{$80 / 20$} & 0.25 & 23 & 0.23 & 0.37 & 26 & 22 & 0.87 & 0.13 \\
\hline & 0.30 & 32 & 0.28 & 0.47 & 32 & 31 & 0.88 & 0.12 \\
\hline & 0.35 & 38 & 0.33 & 0.50 & 41 & 36 & 0.86 & 0.14 \\
\hline & 0.40 & 46 & 0.38 & 0.53 & 52 & 43 & 0.85 & 0.15 \\
\hline \multirow[t]{4}{*}{$65 / 35$} & 0.25 & 22 & 0.22 & 0.33 & 25 & 20 & 0.74 & 0.26 \\
\hline & 0.30 & 29 & 0.25 & 0.45 & 29 & 29 & 0.78 & 0.22 \\
\hline & 0.35 & 34 & 0.31 & 0.48 & 37 & 33 & 0.75 & 0.25 \\
\hline & 0.40 & 42 & 0.36 & 0.51 & 48 & 38 & 0.73 & 0.27 \\
\hline \multirow[t]{4}{*}{$50 / 50$} & 0.25 & 20 & 0.20 & 0.33 & 23 & 19 & 0.62 & 0.38 \\
\hline & 0.30 & 27 & 0.23 & 0.44 & 26 & 27 & 0.67 & 0.33 \\
\hline & 0.35 & 31 & 0.28 & 0.46 & 33 & 30 & 0.63 & 0.37 \\
\hline & 0.40 & 40 & 0.33 & 0.51 & 41 & 40 & 0.62 & 0.38 \\
\hline \multirow[t]{4}{*}{$35 / 65$} & $0.25^{*}$ & 18 & 0.18 & 0.31 & 20 & 19 & 0.49 & 0.51 \\
\hline & 0.30 & 24 & 0.20 & 0.40 & 23 & 24 & 0.53 & 0.47 \\
\hline & 0.35 & 27 & 0.27 & 0.41 & 32 & 25 & 0.46 & 0.54 \\
\hline & 0.40 & 33 & 0.32 & 0.46 & 40 & 30 & 0.45 & 0.55 \\
\hline \multirow[t]{4}{*}{$20 / 80$} & $0.25^{*}$ & 17 & 0.15 & 0.30 & 17 & 18 & 0.35 & 0.65 \\
\hline & 0.30 & 21 & 0.20 & 0.34 & 22 & 20 & 0.31 & 0.69 \\
\hline & 0.35 & 25 & 0.25 & 0.39 & 29 & 23 & 0.28 & 0.72 \\
\hline & 0.40 & 29 & 0.27 & 0.45 & 31 & 30 & 0.31 & 0.69 \\
\hline
\end{tabular}


expected to be negligible, because the phases formed were relatively large with high viscosity. Both assumptions were checked by studying the microstructure of the gels. The results indicated that the experimentally determined $R_{2}$ value of the SPI-WG blend after heating was similar to a concentration based average $R_{2}$ value in which SPI absorbed relatively more water than WG. Hence, the concentration of SPI in its respective phase was lower than the concentration of WG in its respective phase. Table 3.2 shows the experimentally determined $R_{2}$ values of the blend, the corresponding mass fractions of SPI and WG in their respective phases and the phase volumes of SPI and WG after water redistribution.

First, it must be noted that in a few cases there was no match between the summed $R_{2}$ value of the blend and the experimentally determined $R_{2}$ value, because the experimentally determined $R_{2}$-value was lower than the predicted $R_{2}$ value for all theoretical water distributions. This was the case for samples with 25 wt.\% of proteins with a SPI/WG ratio of 20/80 and 35/65. In these samples the minimum of the summed $R_{2}$ value was shown in Table 3.2 with the corresponding volume fractions. The fact that the experimentally determined $R_{2}$ values are lower than the calculated $R_{2}$ values means that water was more mobile than what was predicted. This result can be explained by the presence of free water as the gluten is not able to absorb all available water in this sample. For other samples, the water distributed such that the $R_{2}$ values of the two separate phases became similar compared to the values calculated from the mass fractions when water was evenly distributed. Furthermore, the water distributed similarly for all the protein concentrations $(25-40 \mathrm{wt} . \%)$ at one ratio of SPI and WG. A comparison between the mass fraction and the volume fraction revealed that the volume fraction of SPI was always systematically larger than the mass fraction (Fig. 3.2).

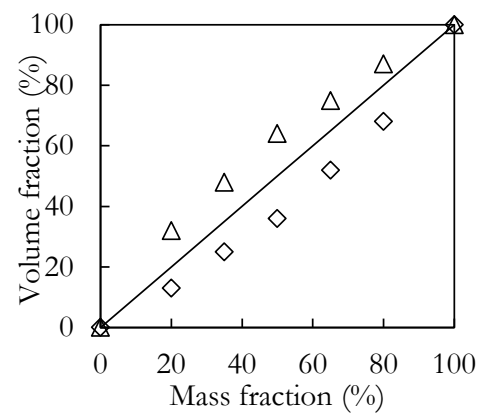

Fig. 3.2 Mass fraction versus volume fraction for $\operatorname{SPI}(\triangle)$ and $W G(\diamond)$ 


\subsubsection{Spatial distribution of SPI and WG with CSLM}

Confocal scanning laser microscopy (CSLM) was used to study the spatial distribution of SPI and WG in the blend. Both the microscope's fluorescence channel and bright field channel were used to differentiate between SPI and WG. Fig. 3.3 shows representative CSLM micrographs of SPI, WG and SPI - WG gels stained with rhodamine B. With the bright field channel, we could differentiate between SPI and WG because the amount of light that could pass through each sample was different for SPI and WG. WG was more opaque, absorbed more light and therefore a darker colour was observed in the bright field channel, whereas the SPI-gel was more translucent, resulting in more light passing through the sample. With the red channel, the fluorescence intensity after staining the proteins with rhodamine B can be analysed. We found that both SPI and WG fluoresced after staining with rhodamine B. However, differences in fluorescence intensity were observed in the SPI-WG blends, which corresponded to the differences in brightness between SPI and WG. The differences in the red channel between SPI and WG originated from differences in affinity of the rhodamine B to the proteins as well as a difference in protein concentration in each phase. It was concluded that a darker phase in the bright field channel and with a higher intensity in the red channel is richer in protein, and therefore was considered to be WG. Consequently, the other phase being lighter in the bright field channel and with a lower intensity in the red channel is lower in protein and thus rich in SPI. The blended gels that were high in WG (ratio 20/80) had a microstructure that contained mainly areas with an intense red colour and a few small areas with a lower intensity of red colour. We concluded that these gels are continuous in WG with dispersed SPI domains. For gels with a ratio of 35/65 SPI and WG, we found that both phases (SPI and WG) were present throughout the whole sample in approximately the same quantity, which indicated the formation of a bicontinuous network. Gels with higher concentrations of SPI (i.e. 50/50, 65/35 and 80/20) seemed to consist of a continuous SPI phase and a dispersed WG phase.

Based on the micrographs, we concluded that the SPI surface fraction in the network was always larger than the corresponding mass fraction. When examining a gel with a ratio of 50/50 SPI and WG for example, the fraction of the WG-phase in the image was smaller compared to the SPI-phase. This corresponds to the findings obtained with TD-NMR (Fig. 3.2). 


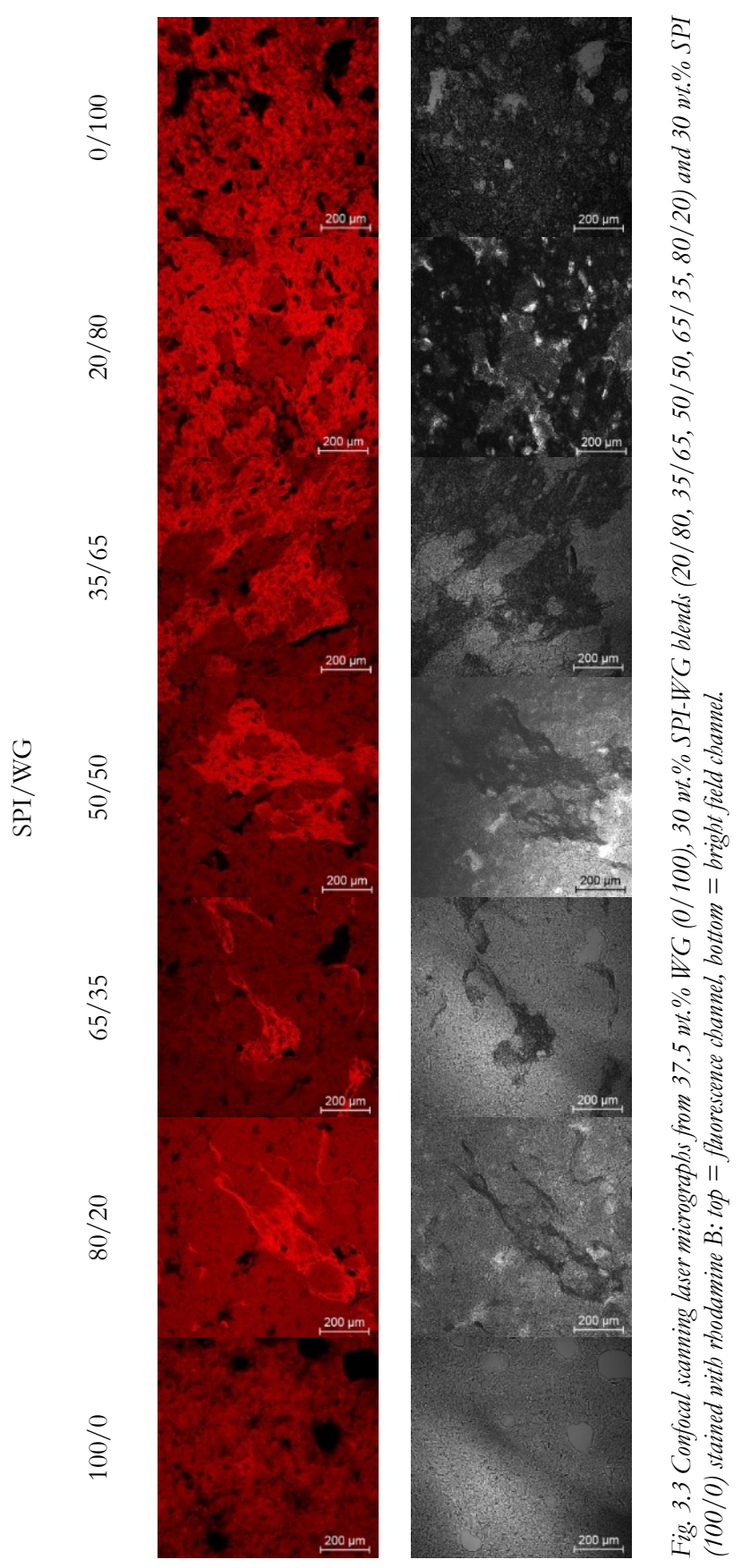




\subsubsection{Rheological properties of SPI and WG}

Whether a two-phase system forms fibrous structures depends on the deformability of the two components during shear-induced structuring. To gain insight in the deformability of the two phases, we performed three types of rheological measurements on SPI, WG and SPI - WG blends. First, the rheological properties of SPI, WG, and SPI-WG were measured at $95^{\circ} \mathrm{C}$ during a time sweep experiment $(15 \mathrm{~min})$ and a frequency sweep in a closed cavity rheometer. With this measurement, the structural changes of SPI, WG and the blend were studied over time while sheared and heated. In addition, a stress-controlled rheometer was used to determine the rheological properties of gelled samples with a frequency sweep. In Fig. 3.4A, typical results of the frequency sweep after the time-sweep are shown for a protein content of $40 \mathrm{wt} . \%$. It is shown that there was hardly any influence of the frequency. In Fig. 3.4B, typical frequency sweeps are also shown for the gels (40 wt.\%). For the gels, a slight frequency dependency was observed. The dry matter content or ratio between SPI and WG did not change the shape of these curves; G' only showed an overall increase or decrease.
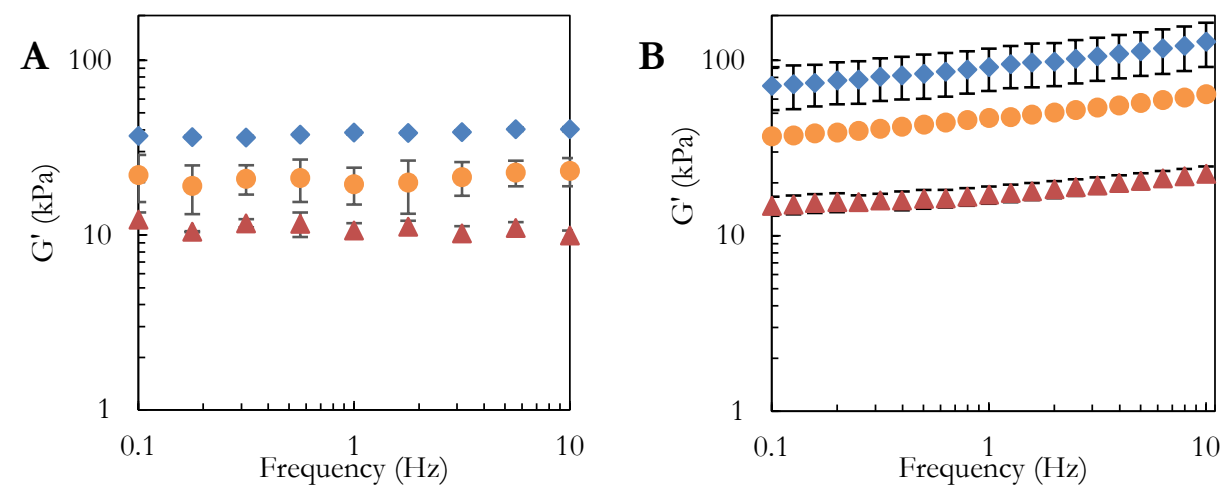

Fig. 3.4 Storage modulus $\left(G^{\prime}\right)$ plotted with standard deviations for SPI $(\diamond), W G(\mathbf{\Delta})$, SPI-WG (50-50) gels $(\mathbf{(})$ measured with a frequency sweep $(10-0.1 \mathrm{~Hz}, 1 \%)$ A) performed after the time-sweep experiment, and B) performed on gels.

In Fig. 3.5A, we show as an example of the time sweep results, i.e. reaction behaviour, of both SPI and WG over time for a protein concentration of $40 \mathrm{wt} . \%$. Heating of SPI from room temperature to $95^{\circ} \mathrm{C}$ caused slight lowering of the apparent $G$ ' during the first minute. Thereafter, the apparent $G^{\prime}$ was almost constant. Different behaviour was seen for WG; the apparent $G$ ' increased over time, which was explained by aggregation or polymerization of the proteins in an earlier study (Pietsch, Emin, \& Schuchmann, 2016). In addition, we calculated the apparent $G_{S P I-W G}^{\prime}$ according to the isostrain $(\mathrm{n}=1)$ and isostress $(\mathrm{n}=-1)$ model when assuming water distributes evenly $\left(\Phi_{S P I}=50\right.$ and $\left.\Phi_{W G}=50\right)$. From the confocal scanning laser 
micrographs, it is known that SPI forms the continuous and WG the dispersed phase in a 50-50 blend. Theoretically, the isostrain model should therefore apply, because soy protein, being the continuous phase, has a higher modulus compared to the dispersed gluten phase. However, the calculated isostrain $G_{S P I-W G}^{\prime}$ overestimates the experimentally measured $G_{S P I-W G}^{\prime}$.

In Fig. 3.5B, we therefore also showed the apparent $G_{S P I}^{\prime}$ and $G_{W G}^{\prime}$ and the calculated $G_{S P I-W G}^{\prime}$ when water distributes unevenly, according to the predictions made with TD-NMR. Now, the apparent $G_{W G}^{\prime}(50 \mathrm{wt} . \%)$ is slightly higher than the apparent $G_{S P I}^{\prime}(33 \mathrm{wt} . \%)$. The calculated $G_{S P I-}^{\prime}$ ${ }_{W G}$ according to the isostrain and isostress model became quite similar. Here, we found that the experimental apparent $G^{\prime}$ of the blend was similar to the calculated apparent $G_{S P I-W G}^{\text {(after } 15}$ min) according to the isostrain model.
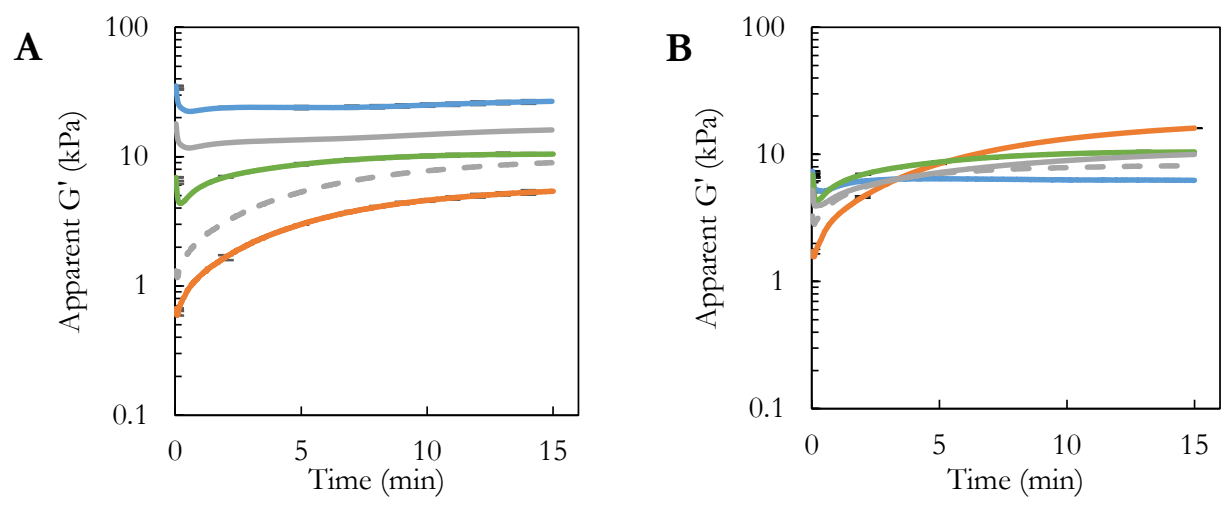

Fig. 3.5 Apparent storage modulus (G') measured during a time sweep at high strain $(80 \%)$ and high frequency $(10 \mathrm{~Hz})$ and the apparent storage modulus $\left(G^{\prime}\right)$ calculated based on the isostrain (solid line) and isostress (dashed line) models: A) 40 $w t . \%$ SPI (一), 40 wt.\% WG (一), and 40 wt.\% SPI-WG (50-50) (一), calculated 40 wt.\% SPI-WG (50-50) based on mass fraction (50-50) with power law B) $33 \mathrm{wt} \%$ SPI (-), $50 \mathrm{wt} \% \mathrm{WG}(-)$, and $40 \mathrm{wt} . \%$ SPI-WG (50-50) (一), calculated 40 wt.\% SPI-WG (50-50) based on volume fraction (0.62-0.38) with power laws (-, solid line is isostrain and dashed line is isostress), standard deviation is plotted every min.

In Fig. 3.6, we show the concentration dependency and power law fits for the three different rheological measurements. From all these different rheological experiments, similar conclusions can be drawn regarding the difference in rheological properties between SPI and WG. First, SPI has a higher (apparent) G' as compared to WG at similar concentrations. Second, the rheological properties could not be measured at the exact same protein concentrations for both SPI and WG, because the dispersions formed were different and could not be characterized in a similar manner. For example, it was not possible to measure the rheological properties for WG samples with a concentration $<35$ wt. $\%$ due to water losses. Also, it was not possible to measure the rheological properties for WG $>50 \mathrm{wt} . \%$, because the samples were not uniform; some parts 
were not completely wetted after gelation. Similar observations were made for SPI at high concentrations (>45 wt.\%). Although the various curves presented in Fig. 3.6 look similar, it must be noted that the absolute $G$ 'values of the different measurements cannot be compared between the three rheological data sets since the testing conditions were vastly different.
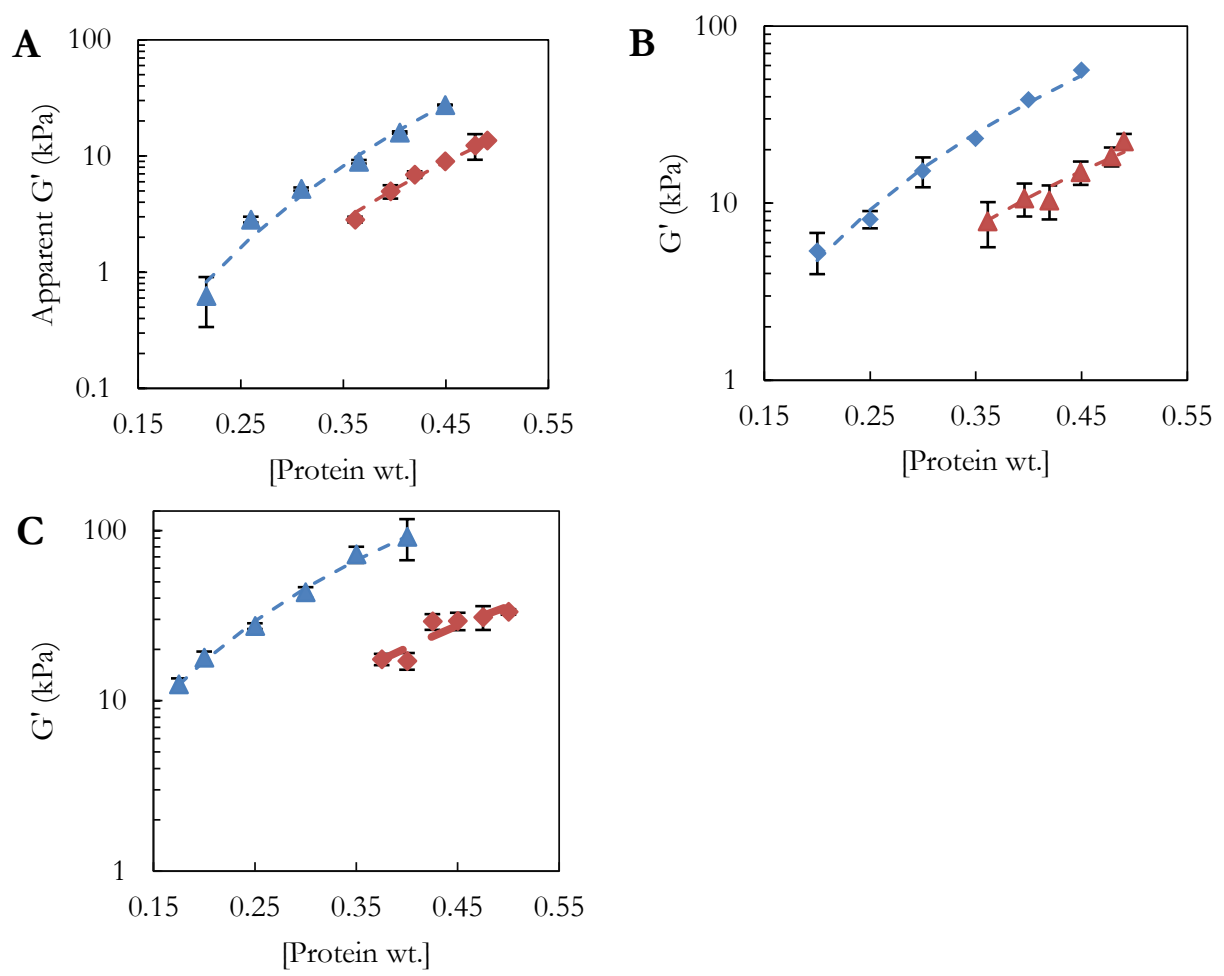

Fig. 3.6 (Apparent) Storage modulus (G') of SPI $(\mathbf{\Delta})$ and WG $(\diamond)$ A) Extracted from a time-sweep experiment after $15 \mathrm{~min}$ (closed symbols), B) Extracted from frequency sweep (10-0.1 Hz, 1\%) performed after time-sweep experiment at 1 $\left.\mathrm{Hz}_{3} \mathrm{C}\right)$ Extracted from frequency sweep (10-0.1 $\left.\mathrm{Hz}, 0.5 \%\right)$ performed on gels at $1 \mathrm{~Hz}$. The data points were fitted with power laws (dashed lines).

The power law relation was subsequently used to calculate the (apparent) G' of all the different blends at various concentrations in two ways: i) for even water distribution into each protein fraction (mass fraction), and ii) according to the water distribution as determined with TD-NMR (volume fraction). The data is shown in supplementary information. Overall, the difference between the mass fraction based (apparent) G' of SPI and WG was larger than that calculated for the volume fraction based (apparent) G' of SPI and WG. In other words, the $G_{S P I}^{\prime}$ and $G_{W G}^{\prime}$

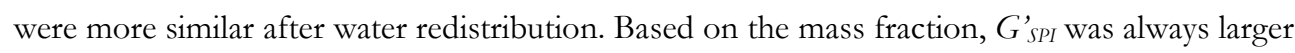
than $G_{W G}^{\prime}$. However, based on the volume fraction, the outcome depended on the rheological method used. For the time-sweep experiment, the $G_{W G}^{\prime}$ was for all protein concentrations and 
ratios larger than $G_{S P I}^{\prime}$. The frequency sweep though (after the time sweep) resulted in small the differences between $G_{S P I}^{\prime}$ and $G_{W G}^{\prime}$. It explains why we found both $G_{S P I}^{\prime}>G_{W G}^{\prime}$ and $G_{S P I}^{\prime}<$ $G_{W G}^{\prime}$. For the frequency sweep performed on the gels, $G_{S P I}^{\prime}$ was mostly larger than $G_{W G}^{\prime}$, which shows that after cooling the soy phase shows more resistance to deformation as compared to the gluten phase.

These $G_{S P I}^{\prime}$ and $G_{W G}^{\prime}$ values were subsequently inserted in the polymer blending law. Since the differences between the (apparent) G'-value of the SPI and WG were quite small, also the differences between the isostrain and isostress power law models were relatively small (Fig. 3.7A). In addition, the G'SPI-WG was experimentally determined with the three rheological methods (Fig. 3.7B). For blends that contain a relatively high amount of gluten $(20 / 80$ and $35 / 65$ ) and a low protein content, some water was expelled during the high strain/frequency treatment, and therefore the values are given at the actual dry matter content. These samples also have a relatively high standard deviation, which is expected to be caused by experimental variation in the water that was expelled. Roughly the same values were obtained for the calculated and experimentally determined (apparent) G' ${ }_{S P I-W G}$ for all three methods. In general, the same, not unexpected, trends can be seen: $i$ ) increased protein concentration resulted in a higher $G^{\prime}$ 'SPIWG, and ii) SPI-WG blends that contained more SPI were closer to the G', wPI, whereas blends that contained more WG in the blend were more similar to $G_{W G}^{\prime}$. Most deviations from these trends were observed for the measurements on the gels that were measured with plate-plate rheology. Especially, the calculated G' ${ }_{S P-W G}$ at higher protein concentration (35 - 40 wt.\%) deviated experimentally from the calculated values for $G_{S P I-W G}^{\prime}$ ones (Fig. 3.7C). Samples containing more WG deviated slightly from the trend that the $G_{S P I-W G}^{\prime}$ increased with protein concentration, mainly at a protein concentration of 35 wt. $\%$. 
A Calculated $G^{\prime}$
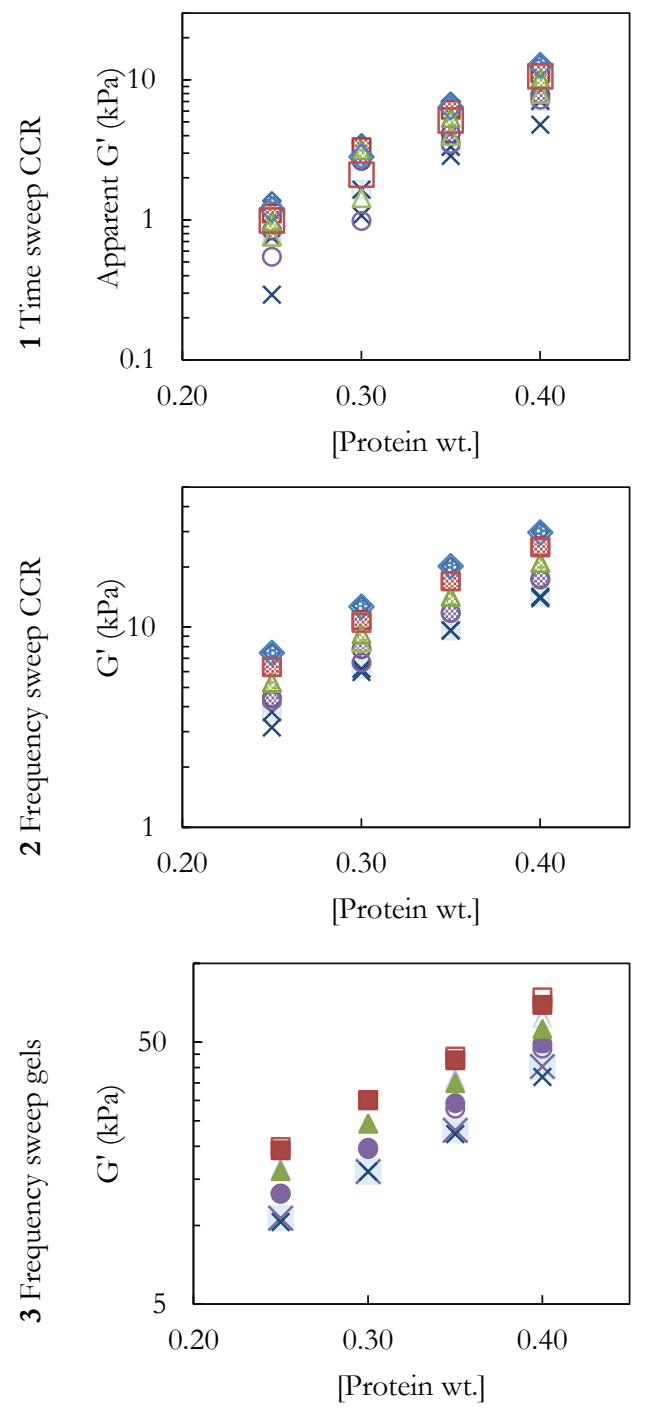

B Experimental $G^{\prime}$
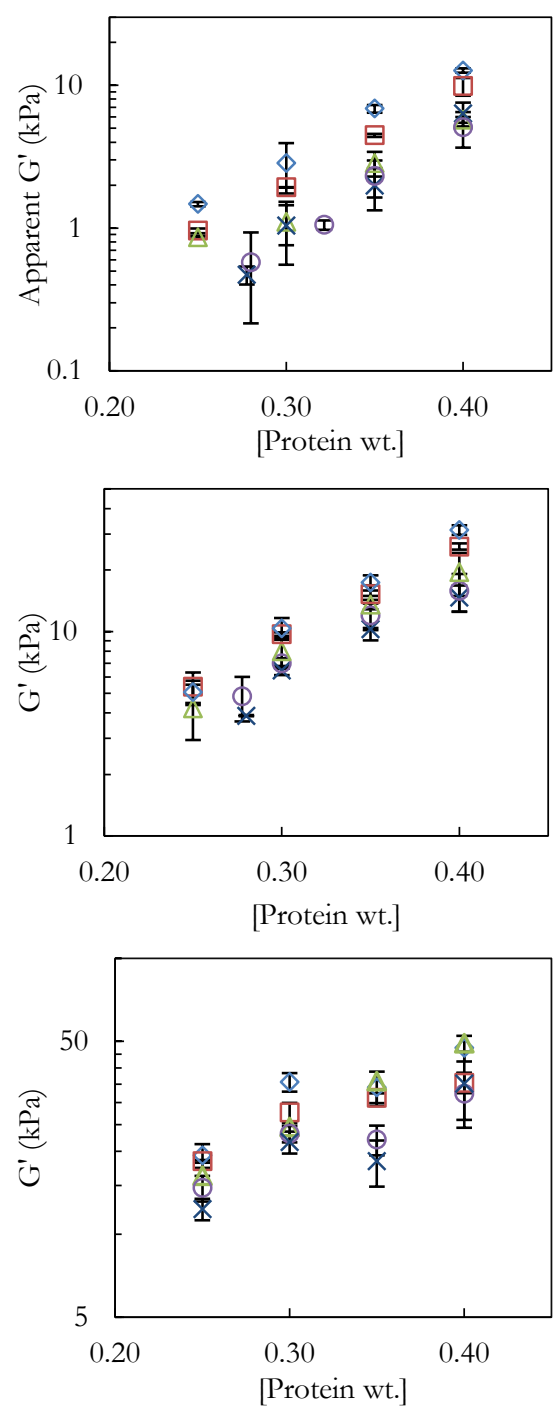

Fig. 3.7 (Apparent) Storage modulus for various concentrations and ratio of SPI-WG blends $(\diamond=80 / 20, \square=65 / 35$, $\triangle=50 / 50, \bigcirc=35 / 65, \times=20 / 80$ mass fraction) A) calculated from power laws based on the volume fraction (closed symbols $=$ isostrain, open symbols $=$ isostress), and B) experimentally determined with 1) time-sweep (15 min), 2) F-sweep performed after time-sweep 3) F-sweep performed on gels with plate-plate rheology. 


\subsection{Conclusion}

In this study, we combined TD-NMR, CSLM and three rheology measurements to determine the phase properties present in concentrated SPI - WG blends. Rheological behaviour was measured using different conditions. It turned out that measurements at process-relevant temperatures provided the most relevant information.

With TD-NMR, it was shown that water is unevenly distributed in SPI - WG blends; more water was absorbed by the SPI phase as compared to the WG phase. However, heating and/or shearing did not affect the water distribution in SPI - WG blend. The water redistribution between SPI and WG was qualitatively confirmed with CSLM. Since the SPI phase contained clearly more water than the WG phase, the volume fraction of SPI in a SPI - WG blend became larger than the mass fraction. This effect resulted in similar rheological properties of the SPIand WG-phases in most blends. These insights in the phase properties of SPI - WG blends can be used to describe other combinations of plant proteins for fibrous structure formation.

\section{Acknowledgements}

The authors wish to thank Daan de Kort for valuable discussions, and Jarno Gieteling for technical support. Nicolien Hendriksen is acknowledged for her contribution to the experimental results. Frank Vergeldt is acknowledged for help with TD-NMR data analysis. 


\section{Supplementary information}

Table S1 Values of G'SPI, G'WG, based on the mass fraction and volume fraction (determined with TD-NMR) calculated with power law dependency of $G$ ' with concentration of protein, and the calculated G'SPI-WG as determined with polymer blending law (isostrain $n=1$ and isostress $n=-1$ ) and the experimental values of G'SPI-WG. G' values are after 15 min timesweep experiment in the CCR.

\begin{tabular}{|c|c|c|c|c|c|c|c|c|c|c|c|c|c|c|c|}
\hline \multicolumn{5}{|c|}{$\begin{array}{l}\text { Mass/Volume } \\
\text { TD-NMR }\end{array}$} & \multicolumn{4}{|l|}{ Mass } & \multicolumn{4}{|c|}{ Volume } & \multicolumn{3}{|l|}{ Exp. } \\
\hline $\begin{array}{l}\text { [Prot- } \\
\text { ein] } \\
\text { (wt) }\end{array}$ & $\mathrm{M}_{\text {SPI }}$ & $\mathrm{M}_{\mathrm{WG}}$ & $\mathrm{V}_{\mathrm{SPI}}$ & $V_{W G}$ & $\mathrm{G}_{\text {SPI }}^{\prime}$ & $\mathrm{G}_{\mathrm{WG}}^{\prime}$ & $\begin{array}{l}\mathrm{G}^{\prime} \\
\text { SPI-WG } \\
\mathrm{n}=1\end{array}$ & 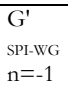 & $G_{\text {SPI }}^{\prime}$ & $G^{\prime}{ }_{W G}$ & $\begin{array}{l}\mathrm{G}_{\text {SPI-WG }}^{\prime} \\
\mathrm{n}=1\end{array}$ & $\begin{array}{l}\mathrm{G}_{\text {SPI-WG }}^{\prime} \\
\mathrm{n}=-1\end{array}$ & G'SPI-WG $^{\prime}$ & \pm & sd \\
\hline 0.25 & 0.20 & 0.80 & 0.35 & 0.65 & 1.59 & 0.41 & 0.65 & 0.48 & 0.12 & 1.09 & 0.76 & 0.29 & 0.50 & \pm & 0.03 \\
\hline 0.25 & 0.35 & 0.65 & 0.49 & 0.51 & 1.59 & 0.41 & 0.82 & 0.55 & 0.34 & 1.31 & 0.84 & 0.54 & 1.15 & \pm & 0.39 \\
\hline 0.25 & 0.50 & 0.50 & 0.62 & 0.38 & 1.59 & 0.41 & 1.00 & 0.65 & 0.57 & 1.63 & 0.97 & 0.75 & 1.08 & \pm & 0.09 \\
\hline 0.25 & 0.65 & 0.35 & 0.74 & 0.26 & 1.59 & 0.41 & 1.18 & 0.79 & 0.85 & 1.87 & 1.11 & 0.99 & 0.92 & \pm & 0.05 \\
\hline 0.25 & 0.80 & 0.20 & 0.87 & 0.13 & 1.59 & 0.41 & 1.36 & 1.01 & 1.08 & 3.24 & 1.36 & 1.19 & 1.25 & \pm & 0.06 \\
\hline 0.30 & 0.20 & 0.80 & 0.31 & 0.69 & 3.90 & 1.06 & 1.62 & 1.24 & 0.51 & 2.16 & 1.64 & 1.07 & 1.63 & \pm & 0.01 \\
\hline 0.30 & 0.35 & 0.65 & 0.53 & 0.47 & 3.90 & 1.06 & 2.05 & 1.42 & 0.57 & 4.94 & 2.62 & 0.98 & 1.73 & \pm & 0.18 \\
\hline 0.30 & 0.50 & 0.50 & 0.67 & 0.33 & 3.90 & 1.06 & 2.48 & 1.66 & 1.02 & 7.43 & 3.14 & 1.43 & 2.03 & \pm & 0.12 \\
\hline 0.30 & 0.65 & 0.35 & 0.78 & 0.22 & 3.90 & 1.06 & 2.90 & 2.01 & 1.73 & 8.76 & 3.30 & 2.11 & 6.54 & \pm & 0.07 \\
\hline 0.30 & 0.80 & 0.20 & 0.88 & 0.12 & 3.90 & 1.06 & 3.33 & 2.53 & 2.56 & 10.62 & 3.54 & 2.82 & 7.30 & \pm & 0.02 \\
\hline 0.35 & 0.20 & 0.80 & 0.28 & 0.72 & 8.29 & 2.35 & 3.54 & 2.74 & 1.67 & 3.97 & 3.32 & 2.86 & 1.10 & \pm & 0.04 \\
\hline 0.35 & 0.35 & 0.65 & 0.46 & 0.54 & 8.29 & 2.35 & 4.43 & 3.13 & 2.40 & 5.62 & 4.14 & 3.47 & 3.63 & \pm & 0.23 \\
\hline 0.35 & 0.50 & 0.50 & 0.63 & 0.37 & 8.29 & 2.35 & 5.32 & 3.66 & 2.96 & 9.33 & 5.33 & 3.97 & 4.72 & \pm & 0.60 \\
\hline 0.35 & 0.65 & 0.35 & 0.75 & 0.25 & 8.29 & 2.35 & 6.21 & 4.39 & 4.32 & 11.52 & 6.11 & 5.11 & 5.08 & \pm & 0.18 \\
\hline 0.35 & 0.80 & 0.20 & 0.86 & 0.14 & 8.29 & 2.35 & 7.10 & 5.50 & 5.85 & 14.38 & 7.01 & 6.36 & 6.49 & \pm & 0.17 \\
\hline 0.40 & 0.20 & 0.80 & 0.31 & 0.69 & 15.96 & 4.69 & 6.94 & 5.46 & 2.31 & 9.13 & 7.04 & 4.79 & 5.25 & \pm & 0.41 \\
\hline 0.40 & 0.35 & 0.65 & 0.45 & 0.55 & 15.96 & 4.69 & 8.63 & 6.22 & 5.44 & 9.77 & 7.84 & 7.21 & 6.48 & \pm & 0.19 \\
\hline 0.40 & 0.50 & 0.50 & 0.62 & 0.38 & 15.96 & 4.69 & 10.32 & 7.24 & 5.95 & 17.32 & 10.23 & 7.90 & 11.15 & \pm & 0.28 \\
\hline 0.40 & 0.65 & 0.35 & 0.73 & 0.27 & 15.96 & 4.69 & 12.01 & 8.66 & 9.45 & 15.83 & 11.17 & 10.60 & 10.42 & \pm & 0.07 \\
\hline 0.40 & 0.80 & 0.20 & 0.85 & 0.15 & 15.96 & 4.69 & 13.70 & 10.77 & 11.95 & 19.80 & 13.10 & 12.69 & 14.56 & \pm & 0.42 \\
\hline
\end{tabular}


Table S2 V alues of G'SPI, G'WG, based on the mass fraction and volume fraction (determined with TD-NMR) calculated with power law dependency of G' with concentration of protein, and the calculated G'SPI-WG as determined with polymer blending law (isostrain $n=1$ and isostress $n=-1$ ) and the experimental values of G'SPI-WG. G' values are determined from frequency sweep performed after a time-sweep experiment in the CCR.

\begin{tabular}{|c|c|c|c|c|c|c|c|c|c|c|c|c|c|c|c|}
\hline \multicolumn{5}{|c|}{$\begin{array}{l}\text { Mass/Volume } \\
\text { TD-NMR }\end{array}$} & \multicolumn{4}{|l|}{ Mass } & \multicolumn{4}{|c|}{ Volume } & \multicolumn{3}{|l|}{ Exp. } \\
\hline $\begin{array}{l}\text { [Prot- } \\
\text { ein] } \\
(\mathrm{wt})\end{array}$ & $\mathrm{M}_{\mathrm{SPI}}$ & $\mathrm{M}_{\mathrm{WG}}$ & $\mathrm{V}_{\mathrm{SPI}}$ & $\mathrm{V}_{\mathrm{WG}}$ & $\mathrm{G}_{\text {SPI }}^{\prime}$ & $G_{W G}^{\prime}$ & $\begin{array}{l}\mathrm{G}^{\prime} \\
\text { SPI-WG } \\
\mathrm{n}=1\end{array}$ & $\begin{array}{l}\mathrm{G}^{\prime} \\
\text { sPI-WG } \\
\mathrm{n}=-1\end{array}$ & $G_{\text {SPI }}^{\prime}$ & $G_{W G}^{\prime}$ & $\begin{array}{l}\mathrm{G}^{\prime} \text { SPI-WG } \\
\mathrm{n}=1\end{array}$ & $\begin{array}{l}\text { G'SPI-WG } \\
\mathrm{n}=-1\end{array}$ & G'SPI-WG & \pm & sd \\
\hline 0.25 & 0.20 & 0.80 & 0.35 & 0.65 & 9.20 & 2.75 & 4.04 & 3.20 & 1.94 & 4.74 & 3.77 & 3.16 & 3.88 & \pm & 0.03 \\
\hline 0.25 & 0.35 & 0.65 & 0.49 & 0.51 & 9.20 & 2.75 & 5.01 & 3.65 & 3.59 & 5.25 & 4.44 & 4.28 & 4.82 & \pm & 1.18 \\
\hline 0.25 & 0.50 & 0.50 & 0.62 & 0.38 & 9.20 & 2.75 & 5.98 & 4.24 & 4.93 & 5.92 & 5.30 & 5.26 & 4.22 & \pm & 1.27 \\
\hline 0.25 & 0.65 & 0.35 & 0.74 & 0.26 & 9.20 & 2.75 & 6.94 & 5.05 & 6.30 & 6.38 & 6.32 & 6.32 & 5.39 & \pm & 0.92 \\
\hline 0.25 & 0.80 & 0.20 & 0.87 & 0.13 & 9.20 & 2.75 & 7.91 & 6.27 & 7.28 & 8.66 & 7.46 & 7.43 & 5.06 & \pm & 0.69 \\
\hline 0.30 & 0.20 & 0.80 & 0.31 & 0.69 & 15.80 & 4.65 & 6.88 & 5.41 & 4.61 & 6.90 & 6.19 & 5.98 & 6.46 & \pm & 0.33 \\
\hline 0.30 & 0.35 & 0.65 & 0.53 & 0.47 & 15.80 & 4.65 & 8.55 & 6.17 & 4.96 & 10.9 & 7.76 & 6.66 & 6.99 & \pm & 0.41 \\
\hline 0.30 & 0.50 & 0.50 & 0.67 & 0.33 & 15.80 & 4.65 & 10.22 & 7.18 & 7.03 & 13.7 & 9.24 & 8.38 & 8.01 & \pm & 1.30 \\
\hline 0.30 & 0.65 & 0.35 & 0.78 & 0.22 & 15.80 & 4.65 & 11.90 & 8.59 & 9.66 & 15.0 & 10.9 & 10.5 & 9.72 & \pm & 0.19 \\
\hline 0.30 & 0.80 & 0.20 & 0.88 & 0.12 & 15.80 & 4.65 & 13.57 & 10.67 & 12.2 & 16.7 & 12.8 & 12.7 & 10.4 & \pm & 1.27 \\
\hline 0.35 & 0.20 & 0.80 & 0.28 & 0.72 & 24.96 & 7.23 & 10.78 & 8.43 & 9.48 & 9.68 & 9.62 & 9.62 & 10.3 & \pm & 0.12 \\
\hline 0.35 & 0.35 & 0.65 & 0.46 & 0.54 & 24.96 & 7.23 & 13.44 & 9.63 & 11.8 & 11.7 & 11.8 & 11.8 & 12.0 & \pm & 2.95 \\
\hline 0.35 & 0.50 & 0.50 & 0.63 & 0.37 & 24.96 & 7.23 & 16.10 & 11.22 & 13.4 & 15.5 & 14.2 & 14.1 & 13.6 & \pm & 0.72 \\
\hline 0.35 & 0.65 & 0.35 & 0.75 & 0.25 & 24.96 & 7.23 & 18.75 & 13.43 & 16.8 & 17.5 & 17.0 & 17.0 & 15.3 & \pm & 1.57 \\
\hline 0.35 & 0.80 & 0.20 & 0.86 & 0.14 & 24.96 & 7.23 & 21.41 & 16.75 & 20.2 & 19.8 & 20.1 & 20.1 & 17.4 & \pm & 1.48 \\
\hline 0.40 & 0.20 & 0.80 & 0.31 & 0.69 & 37.08 & 10.61 & 15.91 & 12.38 & 11.5 & 15.4 & 14.2 & 13.9 & 14.7 & \pm & 2.14 \\
\hline 0.40 & 0.35 & 0.65 & 0.45 & 0.55 & 37.08 & 10.61 & 19.88 & 14.15 & 19.3 & 16.0 & 17.5 & 17.3 & 15.8 & \pm & 3.32 \\
\hline 0.40 & 0.50 & 0.50 & 0.62 & 0.38 & 37.08 & 10.61 & 23.85 & 16.50 & 20.4 & 21.9 & 21.0 & 21.0 & 19.6 & \pm & 4.67 \\
\hline 0.40 & 0.65 & 0.35 & 0.73 & 0.27 & 37.08 & 10.61 & 27.82 & 19.80 & 27.0 & 20.8 & 25.4 & 25.0 & 26.1 & \pm & 0.92 \\
\hline 0.40 & 0.80 & 0.20 & 0.85 & 0.15 & 37.08 & 10.61 & 31.79 & 24.74 & 31.1 & 23.6 & 30.0 & 29.7 & 31.5 & \pm & 1.69 \\
\hline
\end{tabular}


Table S3 V alues of G'SPI, G'WG, based on the mass fraction and volume fraction (determined with TD-NMR) calculated with power law dependency of $G^{\prime}$ with concentration of protein, and the calculated $G_{S P I-W G}^{\prime}$ as determined with polymer blending law (isostrain $n=1$ and isostress $n=-1$ ) and the experimental values of $G$ 'SPI-WG. G' values are determined from frequency sweep performed on gels.

\begin{tabular}{|c|c|c|c|c|c|c|c|c|c|c|c|c|c|c|c|}
\hline \multicolumn{5}{|c|}{$\begin{array}{l}\text { Mass/Volume } \\
\text { TD-NMR }\end{array}$} & \multicolumn{4}{|l|}{ Mass } & \multicolumn{4}{|c|}{ Volume } & \multicolumn{3}{|l|}{ Exp. } \\
\hline $\begin{array}{l}\text { [Prot- } \\
\text { ein] } \\
\text { (wt) }\end{array}$ & $\mathrm{M}_{\mathrm{SPI}}$ & $\mathrm{M}_{W G}$ & $\mathrm{~V}_{\text {SPI }}$ & $\mathrm{V}_{\mathrm{WG}}$ & $\mathrm{G}_{\text {SPI }}^{\prime}$ & $\mathrm{G}_{\mathrm{WG}}^{\prime}$ & 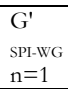 & $\begin{array}{l}\mathrm{G}^{\prime} \\
\text { sPI-WG } \\
\mathrm{n}=-1 \\
\end{array}$ & $\mathrm{G}_{\mathrm{SPI}}^{\prime}$ & $G_{\text {WG }}^{\prime}$ & $\begin{array}{l}\text { G'SPI-WG } \\
\mathrm{n}=1\end{array}$ & $\begin{array}{l}\mathrm{G}^{\prime} \text { 'SPI-WG } \\
\mathrm{n}=-1\end{array}$ & G'SPI-WG & \pm & sd \\
\hline 0.25 & 0.20 & 0.80 & 0.35 & 0.65 & 29.5 & 7.94 & 12.2 & 9.30 & 8.23 & 11.9 & 10.6 & 10.3 & 12.3 & \pm & 1.10 \\
\hline 0.25 & 0.35 & 0.65 & 0.49 & 0.51 & 29.5 & 7.94 & 15.5 & 10.7 & 13.6 & 12.8 & 13.2 & 13.2 & 14.7 & \pm & 1.57 \\
\hline 0.25 & 0.50 & 0.50 & 0.62 & 0.38 & 29.5 & 7.94 & 18.7 & 12.5 & 17.7 & 14.0 & 16.3 & 16.1 & 20.0 & \pm & 3.45 \\
\hline 0.25 & 0.65 & 0.35 & 0.74 & 0.26 & 29.5 & 7.94 & 21.9 & 15.1 & 21.6 & 14.8 & 19.8 & 19.3 & 18.4 & \pm & 0.21 \\
\hline 0.25 & 0.80 & 0.20 & 0.87 & 0.13 & 29.5 & 7.94 & 25.1 & 19.1 & 24.3 & 18.6 & 23.6 & 23.4 & 19.3 & \pm & 1.91 \\
\hline 0.30 & 0.20 & 0.80 & 0.31 & 0.69 & 45.9 & 11.7 & 18.5 & 13.8 & 16.7 & 15.7 & 16.0 & 16.0 & 21.6 & \pm & 1.97 \\
\hline 0.30 & 0.35 & 0.65 & 0.53 & 0.47 & 45.9 & 11.7 & 23.7 & 15.8 & 17.7 & 22.1 & 19.8 & 19.5 & 23.1 & \pm & 1.58 \\
\hline 0.30 & 0.50 & 0.50 & 0.67 & 0.33 & 45.9 & 11.7 & 28.8 & 18.7 & 23.6 & 26.1 & 24.4 & 24.4 & 24.6 & \pm & 3.12 \\
\hline 0.30 & 0.65 & 0.35 & 0.78 & 0.22 & 45.9 & 11.7 & 33.9 & 22.7 & 30.6 & 27.9 & 30.0 & 30.0 & 27.6 & \pm & 2.33 \\
\hline 0.30 & 0.80 & 0.20 & 0.88 & 0.12 & 45.9 & 11.7 & 39.0 & 29.0 & 37.2 & 30.2 & 36.4 & 36.2 & 35.6 & \pm & 2.75 \\
\hline 0.35 & 0.20 & 0.80 & 0.28 & 0.72 & 66.7 & 16.3 & 26.3 & 19.2 & 30.2 & 20.2 & 23.0 & 22.3 & 18.4 & \pm & 3.49 \\
\hline 0.35 & 0.35 & 0.65 & 0.46 & 0.54 & 66.7 & 16.3 & 33.9 & 22.1 & 36.1 & 23.3 & 29.2 & 27.8 & 22.0 & \pm & 2.72 \\
\hline 0.35 & 0.50 & 0.50 & 0.63 & 0.37 & 66.7 & 16.3 & 41.5 & 26.1 & 40.0 & 28.7 & 35.8 & 34.9 & 34.9 & \pm & 4.70 \\
\hline 0.35 & 0.65 & 0.35 & 0.75 & 0.25 & 66.7 & 16.3 & 49.1 & 32.0 & 48.3 & 31.3 & 44.1 & 42.5 & 31.1 & \pm & 1.27 \\
\hline 0.35 & 0.80 & 0.20 & 0.86 & 0.14 & 66.7 & 16.3 & 56.6 & 41.2 & 56.1 & 34.2 & 53.2 & 51.7 & 34.0 & \pm & 4.85 \\
\hline 0.40 & 0.20 & 0.80 & 0.31 & 0.69 & 92.3 & 21.6 & 35.7 & 25.5 & 35.4 & 28.4 & 30.6 & 30.3 & 35.1 & \pm & 10.79 \\
\hline 0.40 & 0.35 & 0.65 & 0.45 & 0.55 & 92.3 & 21.6 & 46.4 & 29.5 & 54.1 & 29.2 & 40.3 & 36.8 & 32.2 & \pm & 6.26 \\
\hline 0.40 & 0.50 & 0.50 & 0.62 & 0.38 & 92.3 & 21.6 & 57.0 & 35.0 & 56.6 & 37.0 & 49.2 & 47.2 & 47.0 & \pm & 2.99 \\
\hline 0.40 & 0.65 & 0.35 & 0.73 & 0.27 & 92.3 & 21.6 & 67.6 & 43.0 & 71.2 & 35.6 & 61.7 & 56.1 & 35.4 & \pm & 2.91 \\
\hline 0.40 & 0.80 & 0.20 & 0.85 & 0.15 & 92.3 & 21.6 & 78.2 & 55.8 & 80.0 & 39.1 & 74.0 & 69.4 & 47.3 & \pm & 5.05 \\
\hline
\end{tabular}




\section{Chapter 4}

Shear-induced fibrous structure formation from a soy
protein isolate - pectin blend

This chapter has been published as B.L. Dekkers, C.V. Nikiforidis, A.J. van der Goot (2016). Shear-induced fibrous structure formation from a pectin / SPI blend. Innovative Food Science and Emerging Technologies, 36, 193-200. http://doi.org/10.1016/j.ifset.2016.07.003 


\section{Abstract}

Well-defined shear flow can be applied to create fibrous, anisotropic samples from biopolymers when present at sufficiently high dry matter contents. Anisotropic biopolymer structures are of high interest especially when made from plant-based polymer blends due to novel food applications, like meat replacers. We investigate shear-induced structuring of a pectin/soy protein isolate (SPI) blend under heating. Scanning electron microscope analysis revealed that shear-induced structuring resulted in elongated pectin filaments, oriented in the direction of the shear flow, being entrapped in a continuous protein phase, inducing anisotropy in the blend. The length of the pectin filaments increased upon higher pectin concentrations and shearing temperatures, leading both to higher anisotropy, as measured with the tensile strength analysis. The fibrous appearance of samples became more evident when deforming the product by tearing, which effect was thought to be caused by detachment through or along the long side of the pectin filament. 


\subsection{Introduction}

The efficient preparation of fibrous products based on plant materials is of interest, because these products can be a starting point for the development of meat replacers. Meat replacers made from plant material are a promising, innovative, and sustainable source of protein for human consumption. With an increasing world population, creation of innovative sources of protein are needed to be able to feed everyone (United Nations - Department of Economic and Social Affairs, 2015). Proteins from plant sources, such as soy, are preferred over proteins from animal origin because plant based materials, for example, have lower environmental impact (Day, 2013; Mogensen, Hermansen, Halberg, Dalgaard, Vis, \& Smith, 2009). In order to replace meat by a plant-based meat replacer, it is important that a similar product in terms of structural properties is developed to improve consumer acceptance (Hoek et al., 2011).

Meat replacers are often produced with extrusion cooking, which is a process that has been applied for texturization of plant materials in the application of meat replacers for decades (Campbell, 1981; Harper \& Clark, 1978). Previous research showed that a novel technique based on well-defined shear flow can also be used to create fibrous, anisotropic structures from plantbased biopolymers at sufficiently high dry matter contents, with a cone-cone device (shear cell) or a concentric cylinder device (couette cell) (Grabowska et al., 2014; Krintiras et al., 2014; Manski et al., 2007b). This novel technique uses milder conditions for structure formation, due to lower applied shear forces, and has therefore a lower specific mechanical energy input (Grabowska et al., 2016; Krintiras, Gadea Diaz, van der Goot, Stankiewicz, \& Stefanidis, 2015). Shear-induced structuring yielded anisotropic structures with starch - zein blends, and fibrous structures were obtained with soy protein isolate (SPI) - wheat gluten blends, and soy protein concentrate (SPC), whereas isotropic structures were obtained for shear-induced structuring of soy protein isolate (SPI) alone (Grabowska et al., 2016, 2014; Habeych, Dekkers, van der Goot, \& Boom, 2008). Studies based on well-defined shear flow and also extrusion studies concluded that a blend with thermodynamically incompatible biopolymers, yielding phase separation was prerequisite for anisotropic structure formation (Cheftel et al., 1992; Grabowska et al., 2016, 2014). However the exact formation mechanism within these biopolymer blends under shear flow while heating is not fully understood yet.

The temperature at which the blends are deformed is important for anisotropic structure formation. Higher temperatures induces lower viscosity, unfolding of proteins, higher flexibility of polymers, which all can have an indirect effect on the extent of phase separation and 
deformability of the two phases (Grinberg \& Tolstoguzov, 1997; A.M. Hermansson, 1985; Thakur, Singh, \& Handa, 1997; Tolstoguzov, 1998). Besides, heat-set proteins form a solid gel after heating and subsequent cooling, which can be used to entrap the anisotropic structure formed upon shear-induced structuring. The use of a process based on well-defined shear flow, enabled us to study the effect of temperature highly controlled due to low viscous dissipation in that process (Grabowska et al., 2016).

In this study, we present shear-induced structuring with well-defined shear flow of a plant-based model biopolymer blend that consists of a protein phase (SPI), and a well-defined dispersed polysaccharide phase (pectin). Pectin is a soluble polysaccharide consisting of 1,4 -linked $\alpha$-Dgalacturonic acid (Lau, McNeil, Darvill, \& Albersheim, 1985). Pectin was chosen as the polysaccharide phase, because this is the main soluble polysaccharide fraction of soy protein concentrate (Day, 2013; Karr-Lilienthal, Kadzere, Grieshop, \& Fahey, 2005). In a previous study, we already found that the application of well-defined shear flow to SPC, can result in alignment of the protein and carbohydrate phase in SPC, leading to a fibrous material eventually (Grabowska et al., 2016). The use of pectin/SPI blends offers possibilities to study the effect of shearing on alignment of the protein and the dispersed pectin phase separately by using different ratios of SPI and pectin. Besides, the temperature was varied to study changes in deformability of the two phases. In this paper, we relate the microstructures of these anisotropic samples, by using a scanning electron microscopy (SEM), with the mechanical properties, that were determined by uniaxial tensile strength analysis.

\subsection{Materials and methods}

\subsubsection{Materials}

Soy protein isolate (SUPRO ${ }^{\circledR}$ 500E IP) was obtained from Solae (St Louis, MO, USA). The isolate, contained at least $90 \mathrm{wt} . \%$ protein ( $\mathrm{N}$ 6.25). Pectin from citrus peel (P9135), sodium chloride (S9625), and glutaraldehyde (25 v.\%) were obtained from Sigma-Aldrich (Germany, Darmstadt). Acetone ( $99 \%$ purity) was purchased from Actu-All Chemicals (Oss, The Netherlands). The particle size distributions of soy protein isolate $\left(\mathrm{d}_{0.1}=12.5 \pm 0.2, \mathrm{~d}_{0.5}=38.3\right.$ $\left.\pm 1.2, \mathrm{~d}_{0.9}=138.0 \pm 21.8\right)$, and pectin $\left(\mathrm{d}_{0.1}=20.7 \pm 0.5, \mathrm{~d}_{0.5}=78.5 \pm 1.1, \mathrm{~d}_{0.9}=167.3 \pm 1.2\right)$ were determined in triplicate by laser diffraction using a Mastersizer 2000 equipped with the Sciricco 2000 dry dispersion unit (Malvern Instruments, Worcestershire, UK). 


\subsubsection{Methods}

\subsubsection{Preparation of pectin/SPI blends}

Shear-induced structuring of pectin/SPI blends was studied using a constant dry matter content of $45 \mathrm{wt.} \%$. The total weight, therefore, comprised of 1 wt. $\% \mathrm{NaCl}, 44 \mathrm{wt.} \%$ biopolymers and $55 \mathrm{wt} . \%$ demi water. The ratio pectin and SPI was varied by replacing part of SPI with pectin stepwise (1.3 - 4.0 wt. \%, in increments of 0.9 wt. \%). Mixing of the ingredients occurred by dissolving salts in demineralized water first after which SPI was added to the salt solution. A hydration time of 30 min was applied to the dispersion of proteins. Then, pectin was added to the dispersion and mixed thoroughly. This order of hydrating proteins and pectin was found to give the most pronounced fibrous structures, and was therefore used.

\subsubsection{Shear-induced structuring with a high temperature shear cell}

A high temperature shear cell (Wageningen University, The Netherlands) was used to structure the pectin/SPI blends into anisotropic samples. The shear cell was developed in house and its design was based on previously reported equipment (Habeych et al., 2008; Manski et al., 2007b; Peighambardoust, van der Goot, Hamer, \& Boom, 2004; Zalm, Goot, \& Boom, 2009). Adaptation of this device was done to be able to process at high temperatures $\left(100{ }^{\circ} \mathrm{C}-150{ }^{\circ} \mathrm{C}\right)$ (Grabowska et al., 2016). The shear cell consists of a rotating bottom cone and a stationary cone. An oil bath (JULABO LH46, USA) filled with Thermal H10 oil (JULABO, Germany) was used to heat and cool the cones, while a thermocouple measured the temperature inside the cone. A Haake drive (Haake Polylab QC, Germany) was used to control rotation speed. SPI and pectin/SPI blends were heated at three different temperatures: 120,130 and $140{ }^{\circ} \mathrm{C}$. The blends were put into a shear cell that was preheated to the set temperature, and then sheared for 15 min at $30 \mathrm{rpm}$. The non-sheared samples were just heated in the shear cell for $15 \mathrm{~min}$. The shear cell was subsequently cooled down to $25^{\circ} \mathrm{C}$ within $15 \mathrm{~min}$, after which samples were taken out.

\subsubsection{Scanning Electron Microscopy}

Samples were frozen at $-18{ }^{\circ} \mathrm{C}$ after preparation and were kept at $30 \mathrm{~min}$ at room temperature prior to sample preparation. Structures were cut into small rectangles (the longest edge $13 \mathrm{~mm}$ ) and stored in a glutaraldehyde solution $(2.5 \mathrm{v} . \%)$, while gently rotating for eight hours. Next, samples were soaked in demineralized water overnight. Then, the samples were immersed in a series of solutions of acetone (10, 30, 50, 70 and $100 \mathrm{v} . \%)$ for at least two hours per solution. Samples were dried by critical point drying (CPD 300, Leica, Vienna, Austria). Finally, samples were fractured into two directions to observe the sample surfaces from different angles to reveal 
the anisotropic nature. Samples were attached onto sample holders and sputter coated with a 10 nm thick layer of wolfram (SCD 500, Leica, Vienna, Austria). The surfaces were analyzed with a field emission scanning electron microscope (Magellan 400, FEI, Eindhoven, the Netherlands) at magnifications of 250 times up to 10,000 times at a working distance of $6-8 \mathrm{~mm}$, with SE detection at $2 \mathrm{kV}$ and $6.3 \mathrm{pA}$.

\subsubsection{Tensile strength analysis}

Mechanical properties of the samples were tested with a Texture Analyser (INSTRON 5564, USA). Uniaxial tension tests until failure were performed at room temperature and with a constant deformation of $1 \mathrm{~mm} / \mathrm{s}$. Samples were always analysed approximately one hour after structuring. Specimens were cut using a tensile bar. To determine the degree of anisotropy, samples were cut parallel and perpendicular to the shear direction. The thickness and lower width varied per specimen, therefore we measured these dimensions, and we accounted for this in the tensile stress determination. Both ends of the tensile bars were placed and secured into two clamps, which were coated with sanded paper to prevent slip. Samples were placed such that approximately $15 \mathrm{~mm}$ of sample was outside the clamps, which was the part of the sample where the fracture would occur upon deformation. The force needed to maintain a constant deformation was recorded by the software Blue Hill. The force needed to rupture the samples was divided by the surface area of the specimen to calculate the tensile stress. The deformation was divided by the initial length of the specimen to calculate the tensile strain. The SPI-gel always ruptured at once resulting in a clear yield point. Addition of pectin gave samples that did not rupture at once in some cases. Then, it was decided to take tensile stress and strain from the first large rupture point. Rupturing in more steps indicated that the sample was fibrous though. From the tensile stress and deformation at the rupture, the Anisotropic Indices were calculated with the following equations

$$
\begin{aligned}
& \text { Anisotropic index } \sigma=\frac{\sigma_{\text {parallel }}}{\sigma_{\text {perpendicular }}} \\
& \text { Anisotropic index } x_{\varepsilon}=\frac{\varepsilon_{\text {parallel }}}{\varepsilon_{\text {perpendicular }}}
\end{aligned}
$$

where $\sigma_{\text {parallel }}$ and $\sigma_{\text {perpendicular }}$ are the fracture stress-values in parallel and perpendicular direction and $\varepsilon_{\text {paralle }}$ and $\varepsilon_{\text {perpendicular }}$ are the fracture strain-values in parallel and perpendicular direction. We were able to cut three tensile bars in parallel direction and three tensile bars in perpendicular direction per structured sample. Three structured samples were made and therefore nine replicates were done for the tensile test. The error bar is the standard error between the three structured samples. 


\subsection{Results and Discussion}

A high temperature shear cell was used to make fibrous structures from a pectin/SPI blend. We studied the effect of shearing on alignment of the two phases, SPI and pectin, present at different ratios. Besides, the temperature is explored as process parameter to obtain anisotropic structures with a fibrous appearance. Pre-trials showed that a temperature range between $120-140{ }^{\circ} \mathrm{C}$ resulted in structures that show the transition of isotropic, layered structures to anisotropic, fibrous structures and therefore this temperature range was studied. Samples heated at lower temperatures $\left(<120^{\circ} \mathrm{C}\right)$ were not fibrous and weak and therefore difficult to analyse. Samples heated at higher temperatures $\left(>140{ }^{\circ} \mathrm{C}\right)$ had a darker colour, and preliminary results showed a decrease in anisotropy. The macrostructure and microstructure of samples, and their mechanical properties were thoroughly analysed. The combination of all experimental results was used to formulate new hypothesis on shear-induced structuring of plant-based biopolymer blends for applications in meat replacers.

\subsubsection{Macrostructure}

Different types of sample structures were obtained after shear-induced structuring while heating $\left(120-140^{\circ} \mathrm{C}\right)$ of a pectin/SPI blend. Folded samples of the representative structures are shown in Fig. 4.1. Shear-induced structuring of SPI without pectin yielded a rubbery, layered structure (Fig. 4.1A). Although the colour is slightly darker for samples sheared at $140{ }^{\circ} \mathrm{C}$, the rubbery and layered structure was hardly changed by different shearing temperatures $\left(120-140{ }^{\circ} \mathrm{C}\right)$. When 2.2 wt. $\%$ pectin was added to the sample, it was observed that temperature greatly influenced the final structure of blends (Fig. 4.1C). A layered structure was obtained for samples heated at $120^{\circ} \mathrm{C}$, small fibres were found in samples heated at $130{ }^{\circ} \mathrm{C}$, while pronounced fibres were shown in samples heated at $140{ }^{\circ} \mathrm{C}$. Similar structural changes were observed for SPC when changing the shearing temperature (Grabowska et al., 2016). The concentration of pectin influenced structure formation also. When $1.3 \mathrm{wt} . \%$ of SPI was replaced, a rubbery sample was obtained that was similar to a SPI sample without pectin (Fig. 4.1-B1). Addition of higher concentrations of pectin $(2.2$ - 4.0 wt.\%) resulted in fibrous, but weaker samples (Fig. 4.1-B2B4). 


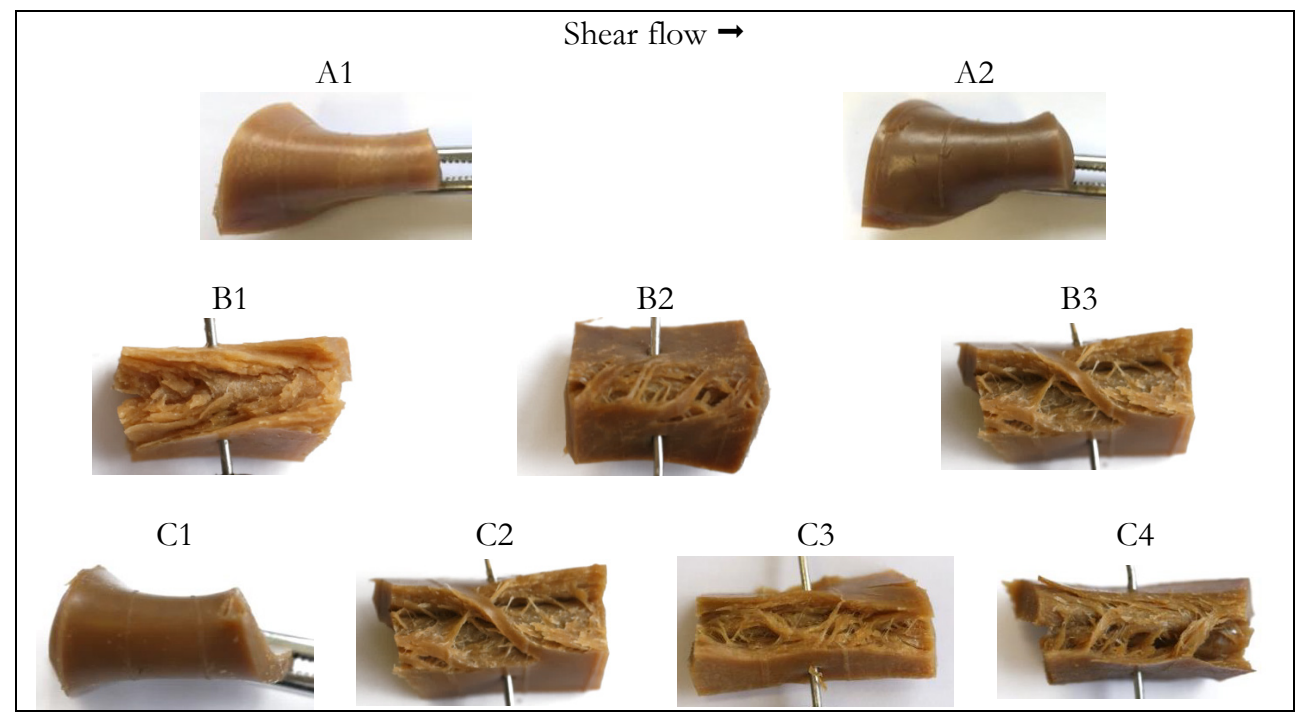

Fig. 4.1 Macrostructures after shear-induced structuring of samples consisting of A) soy protein isolate (SPI) at A1) $120^{\circ} \mathrm{C}$ A2) $140{ }^{\circ} \mathrm{C}$, and B) SPI + $2.2 \mathrm{wt. \%}$ pectin at B1) $120^{\circ} \mathrm{C} \mathrm{B2)} 130^{\circ} \mathrm{C} \mathrm{B3)} 140^{\circ} \mathrm{C}$, and SPI + pectin at $140{ }^{\circ} \mathrm{C} \mathrm{C1}$ ) $1.3 \mathrm{wt. \%}$ pectin C2) $2.2 \mathrm{wt} \%$ pectin C3) $3.1 \mathrm{wt. \%}$ pectin, and C4) SPI +4 wt.\% pectin, , the samples were roughly 25 $\mathrm{mm}$ in width.

\subsubsection{Microstructure}

Microstructures of pectin/SPI blends were studied with a scanning electron microscope (SEM). Fig. 4.2A shows that the microstructure of a shear-induced structured SPI sample without pectin was similar in all directions, i.e. no orientation was found. The surface was smooth at low magnification, while higher magnification (Fig. 4.2B) revealed a dense particulate network, which is typical for a heated SPI gel at neutral pH (Grabowska et al., 2014; Maltais, Remondetto, \& Subirade, 2009). Fig. 4.2C shows a SEM image of a non-sheared sample that consists of a pectin/SPI blend (2.2 wt. $\%$ pectin, heated at $\left.140{ }^{\circ} \mathrm{C}\right)$. A continuous smooth surface with dispersed droplets was observed in the microstructure, probably reflecting pectin droplets in a SPI matrix. The latter was confirmed by the smooth surface, which was similar to Fig. 4.2A in which only SPI was present. The dispersed droplets were not present in the SPI continuous phase and these droplets are, therefore, expected to be mainly pectin. The size range of these droplets was similar to the dry pectin particles. Higher magnification showed a more open morphology for the dispersed phase compared to the rigid continuous phase. The differences in microstructure of the two phases confirmed the different natures of the formed networks and hence the differences in strength and fracture behaviour. SPI forms a cross-linked gel upon heating, whereas pectin contains a crystalline network (Kinsella, 1979; Chen et al., 2015). 

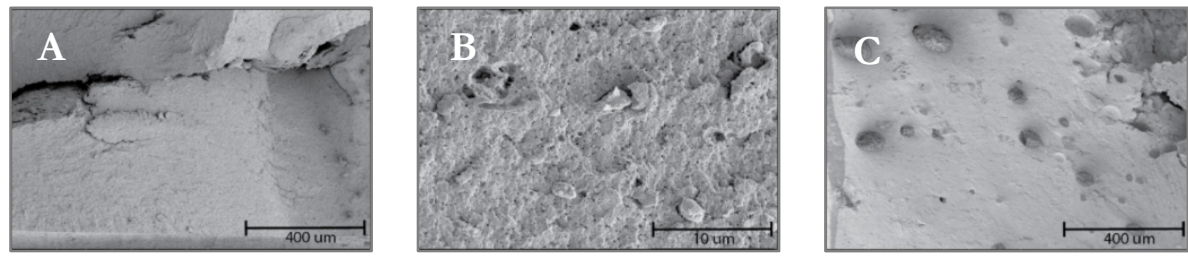

Fig. 4.2 Scanning Electron Microscope images of fractured samples A) and B) sheared for $15 \mathrm{~min}, 30 \mathrm{rpm}$ at $140{ }^{\circ} \mathrm{C}$ at a magnification of 250 times and 10,000 times C) Fractured non-sheared sample with pectin and soy protein isolate (SPI) beated for $15 \mathrm{~min}, 0 \mathrm{rpm}$ at $140{ }^{\circ} \mathrm{C}$ at a magnification of 250 times.

Sheared samples contained elongated droplets along the direction of the applied shear flow ( $\rightarrow$ Shear flow). The fibres that were macroscopically visible were observed in the same direction (Fig. 4.3-1). Spherical droplets were observed when the samples were viewed perpendicularly $(\triangle)$ to the fiber orientation (Fig. 4.3-2). Besides deformation of dispersed droplets, droplet coalescence or further phase separation played a role, because the most filaments were larger compared to the droplets observed in non-sheared samples. Tolstoguzov et al, proposed a similar explanation for fiber formation during spinneretless spinning of biopolymer blends. Phase separation and formation of filaments under shear flow, and long filaments were a result of coalescence of the dispersed fibrils that were initially formed (Tolstoguzov, 1988; Tolstoguzov, Mzhel'sky, \& Gulov, 1974).

The size of filaments and cylindrical droplets changed upon different pectin concentrations (1.3 - 4.0 wt.\%). Samples with 1.3 wt.\% pectin did not contain clear filaments or spherical droplets (Fig. 4.3B). Here, pectin and SPI might be below or close the co-solubility threshold, leading to pectin being mostly dissolved in the SPI phase. Similarly to SPI samples, a smooth surface was found that was disrupted upon fracturing. Samples with $2.2 \mathrm{wt} . \%$ pectin contained several elongated structures (Fig. 4.3A). The use of $3.1 \mathrm{wt} . \%$ pectin gave filaments, having lengths varying from 200 - $750 \mu \mathrm{m}$ but seemed lower in number (Fig. 4.3C). The microstructure of a 4.0 wt. $\%$ pectin sample was different from the microstructures of samples with lower concentrations of pectin. This sample showed many fractures in the shear flow direction, and no pectin filaments were observed. The brittle nature of these samples after drying caused the differences observed and hindered fair comparison with the samples with lower pectin content.

The number, length and width of filaments (parallel to the shear flow), and the diameter of spherical droplets (perpendicular to the shear flow) in samples was also dependent on heating temperature. Heating at 120 and $130{ }^{\circ} \mathrm{C}$ resulted in many, relatively small filaments with an average length of about $100 \mu \mathrm{m}$ (Fig. 4.3D1-E1). The width of filaments was similar to the 
diameter of cylindrical droplets found in microstructures that were viewed perpendicular to the fiber direction, about $10-50 \mu \mathrm{m}$ (Fig. 4.3D2-E2). Samples heated at $140{ }^{\circ} \mathrm{C}$ contained a lower number of filaments with an average length that was approximately twice as long (in the range of $200 \mu \mathrm{m}$ ) compared to 120 and $130^{\circ} \mathrm{C}$. The width of these samples is approximately $70 \mu \mathrm{m}$, as can be seen in Fig. 4.3A2. The effect of temperature on the sample structure is probably a result of viscosity changes of the continuous protein phase and the dispersed polysaccharide phase. Those viscosity changes are probably not only an effect of temperature changes, but could be influenced by chemical changes, such as non-enzymatic degradation of the pectin phase, as well (Diaz, Anthon, \& Barrett, 2007; Fraeye et al., 2007). Non-enzymatic degradation of pectin results in a lower molecular weight of pectin, which might be an explanation of the more open structures of pectin found at $140{ }^{\circ} \mathrm{C}$ (Fig. 4.3B3). Besides, samples viewed at this magnification showed that the interface between pectin and SPI changed with temperature; the interface between dispersed droplets and the continuous SPI phase at 120 and $130{ }^{\circ} \mathrm{C}$ temperature is sharper compared with the interface at $2.2 \mathrm{wt} . \%$ pectin at a temperature of $140{ }^{\circ} \mathrm{C}$ (Fig. $4.3 \mathrm{~A} 3$, D3, and E3). However, this effect was less clearly observed at higher concentrations of pectin (Fig. 4.3C2). Changes in the interface can be a result of more Maillard products that are composed to a higher extent at higher temperatures, and these products are expected to be compatible with both the protein and the pectin phase facilitating increased interaction between both phases (Habeych, van der Goot, \& Boom, 2009; Ledward \& Tester, 1994). 


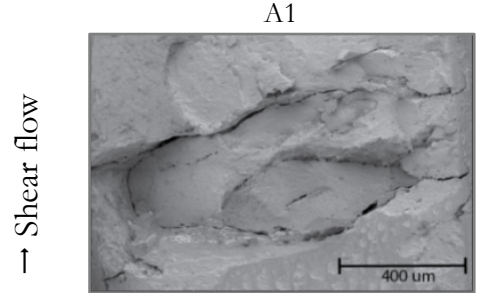

B2
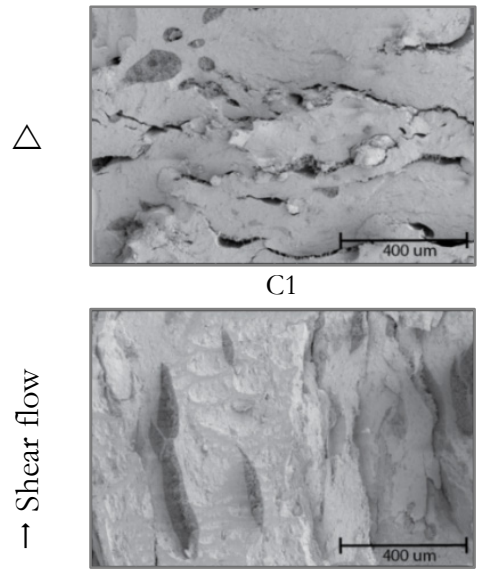

D1

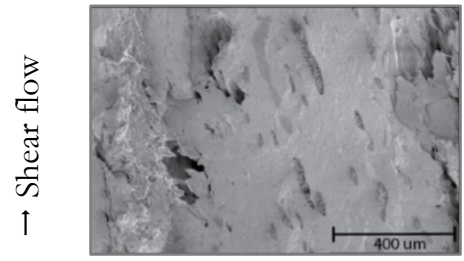

D3

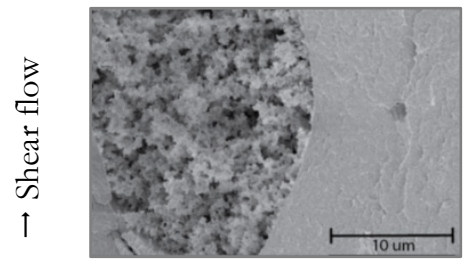

E2

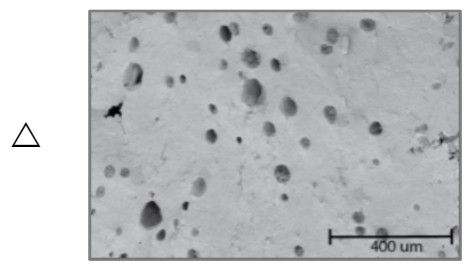

B1

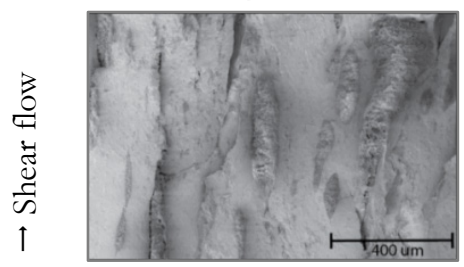

B3

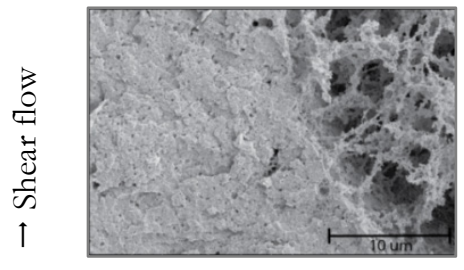

$\mathrm{C} 2$

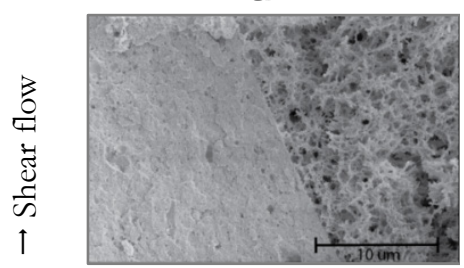

D2

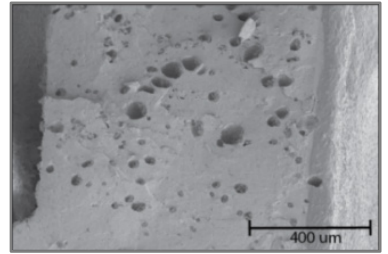

E1

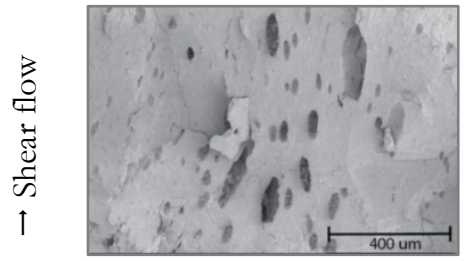

E3

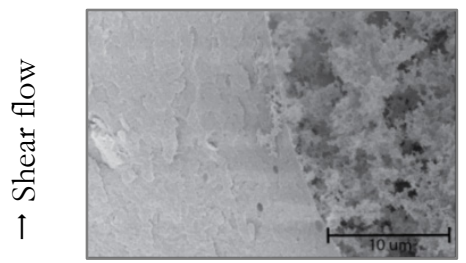

0
0
0
0
1

를

ำ

는

ㄱำ

uิ

0 춘

$1=3$ - $\frac{2}{2}$

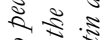

o. के

₹ है

$\therefore$ 을

₹ $\approx \widehat{\bar{\sigma}}$

के

ㄴำ

ก . ำ

촌

in 0

के

구

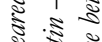

ธ

ํํㄹㅇำ

है ₹

$\therefore \approx$

넌

डิ ०

ฐิ

ह 1 ?

कूत है

औ

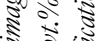

₹

을 तิ

홍

¿

ำ

닌

क 5

는

हैं के

峁

ชे

sio 


\subsubsection{Tensile strength analysis}

The macro- and microstructures of samples show clear anisotropy for pectin/SPI blends with a sufficiently high concentration of pectin $(2.2-4.0 \mathrm{wt} . \%)$ when heated at a sufficiently high temperature $\left(140^{\circ} \mathrm{C}\right)$. We tested the mechanical properties of samples at varying concentrations of pectin, and heating temperatures for further understanding of shear-induced structuring. A tensile strength analysis was performed parallel and perpendicular direction to the shear flow, and the anisotropic index was calculated.

Tensile stress results and the corresponding anisotropic indices for samples with varying concentrations of pectin $(0.0-4.0$ wt.\%) are shown in Fig. 4.4A. The tensile stress for SPI samples was almost similar in parallel and perpendicular direction ( 575 and $545 \mathrm{kPa}$ ). The tensile stress dropped after addition of pectin (1.3 wt.\%) to about 420 and $440 \mathrm{kPa}$ in parallel and perpendicular direction. Lowering of the tensile stress of samples can be a result of two phenomena. In case a small amount of pectin is added to the SPI, pectin can dissolve in the continuous SPI phase. Dissolved pectin influences the ability of SPI to form a network, and therefore decreases the tensile stress. Lowering of the tensile stress as a result of addition of a second component was also observed other types of composite materials (Landon, Lewis, \& Boden, 1977; Sahu \& Broutman, 1972). The further drop in tensile stress at higher concentrations of pectin $(2.2 \mathrm{wt} . \%)$ can be a result of dispersion of pectin in SPI. The pectin phase forms weaker parts or has weak adhesion between pectin and SPI (Habeych et al., 2008). In addition to a lower tensile stress, samples with $2.2 \mathrm{wt} . \%$ pectin resulted in clear differences in tensile stress in parallel direction and perpendicular direction. The parallel tensile stress was shown to be $400 \mathrm{kPa}$, which was similar to the tensile stress at $1.3 \mathrm{wt} . \%$ pectin, whereas the tensile stress in perpendicular decreased to $240 \mathrm{kPa}$. Here, weakening of the samples in perpendicular direction is a result of the dispersed pectin phase, and its morphology and orientation. Weakening of samples, due to the dispersed pectin phase, became even more pronounced at higher pectin concentrations $(3.1-4.0 \mathrm{wt} . \%)$. The differences between parallel and perpendicular outcomes for samples that contain 2.2 and 3.1 wt.\% pectin were similar. Accordingly, a high anisotropic index (1.9) was found for samples that contained 2.2 and 3.1 wt. $\%$ pectin. Higher concentrations of pectin resulted in overall weak structures, and that resulted in low tensile stress in both directions (110 and $70 \mathrm{kPa})$. 


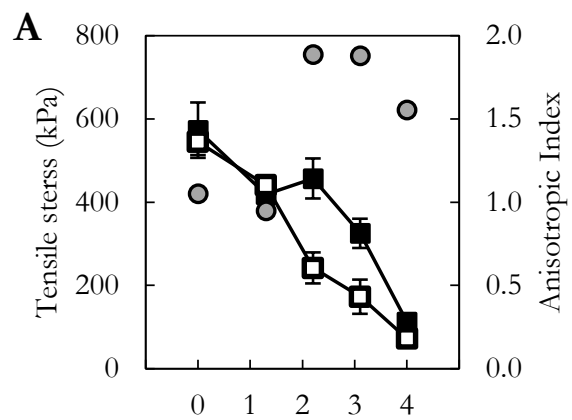

Pectin concentration (wt.\%)

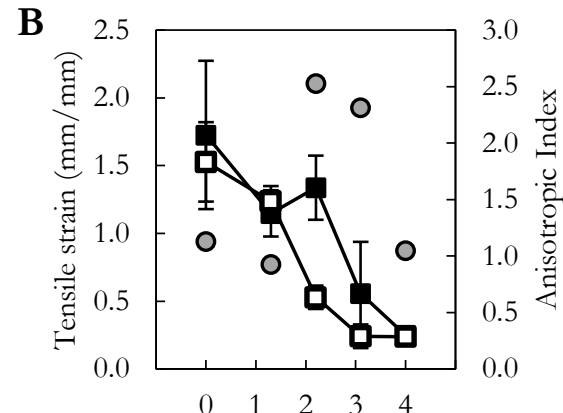

Pectin concentration (wt.\%)

Fig. 4.4 Tensile stress A), tensile strain B) for samples soy protein isolate (SPI) with varying pectin concentrations (0 - 4 wt.\%) sheared for 15 min., $30 \mathrm{rpm}$ at $140^{\circ} \mathrm{C}$ deformed in parallel (ם) and perpendicular $(\mathbf{\square})$ direction, and anisotropic index $(\bullet)$.

The tensile strain values are shown in Fig. 4.4B for samples with varying concentrations of pectin (0.0 - 4.0 wt. \%). High tensile strain values were found for samples that contain 0.0 and 1.3 wt. \% pectin, and the results were similar when these samples were deformed in parallel and perpendicular direction. Tensile strain for samples that contain $2.2 \mathrm{wt} . \%$ was in parallel direction similar to 1.3 wt. $\%$ pectin $(1.3 \mathrm{~mm} / \mathrm{mm})$, whereas the tensile strain in perpendicular direction dropped to $0.5 \mathrm{~mm} / \mathrm{mm}$. The anisotropic index for samples with $2.2 \mathrm{wt} . \%$ was, therefore, much higher (2.5) compared with samples with lower concentrations of pectin. We concluded that addition of $2.2 \mathrm{wt} . \%$ pectin has mainly an influence on the tensile strength in perpendicular direction. A higher pectin concentrations further weakened structures as shown by lower tensile strain values.

Results on tensile stress and strain for the SPI-pectin blends heated at various temperatures are shown in Fig. 4.5. An increase in heating temperature resulted in an increase in tensile stress and tensile strain. Small differences between parallel and perpendicular were found for samples heated at 120 and $130{ }^{\circ} \mathrm{C}$, whereas larger differences were found for samples heated at $140{ }^{\circ} \mathrm{C}$. The anisotropic index for samples heated at 120 and $130{ }^{\circ} \mathrm{C}$ was below 1.5 , while $140{ }^{\circ} \mathrm{C}$ gave higher values. The effect of heating temperature could not directly be ascribed to changes in protein aggregation, since the tensile stress and strain values of SPI samples (44 wt.\%) without pectin did not substantially change (about $550 \mathrm{kPa}$ and 1.68 respectively) when heated at 120 or $140{ }^{\circ} \mathrm{C}$. Hence the weakening of the structures was caused by of the presence of the pectin phase. 

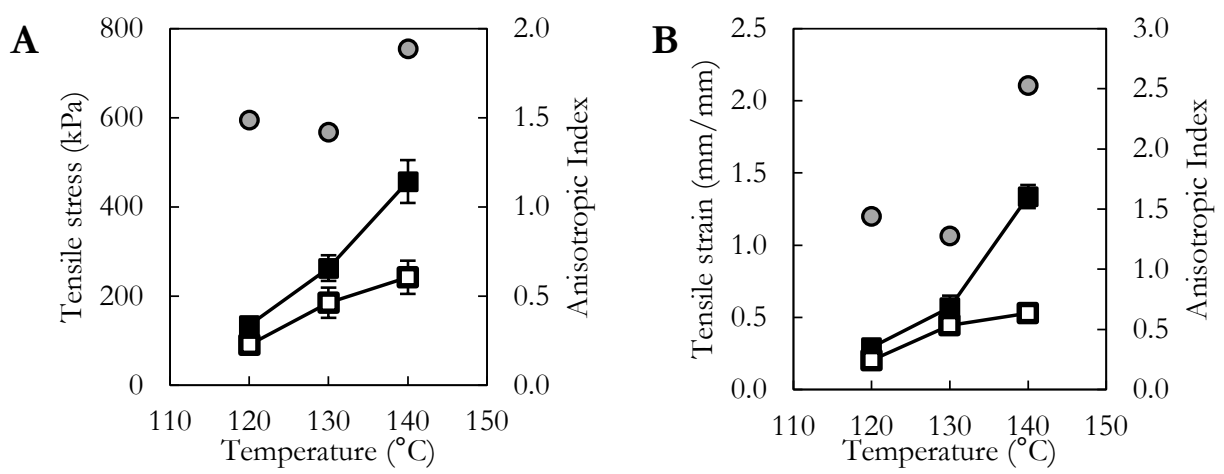

Fig. 4.5 Tensile stress A), tensile strain B) for samples that consist of soy protein isolate (SPI) and $2.2 \mathrm{wt. \%}$ pectin sheared

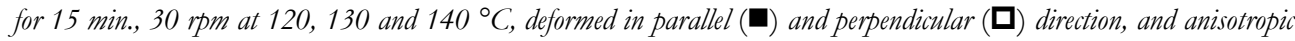
index $(\bullet)$.

\subsubsection{Relating microstructure with mechanical properties}

The macro- and microstructure of the samples were linked to their mechanical properties for further understanding of shear-induced structuring. Fig. 4.6 sketches the relation between the filaments of the dispersed phase and the resulting fracture behaviour. Based on the tensile tests, it is expected that pectin will weaken the overall structure. Weakening of the structure might theoretically be a result of several phenomena such as dissolution of pectin inhibits the formation of a strong SPI network, pectin leads to dilution of the SPI network. We hypothesize that the dispersed pectin forms a weak phase itself, or the adhesion between pectin and SPI is weak (Habeych et al., 2008). Fractures initiated in the product will propagate if the stress is higher than the adhesion or cohesion strength of the material (van Vliet, 1996). Visually observed fibres appear upon tearing the product, and originate from detachment through or along the long side

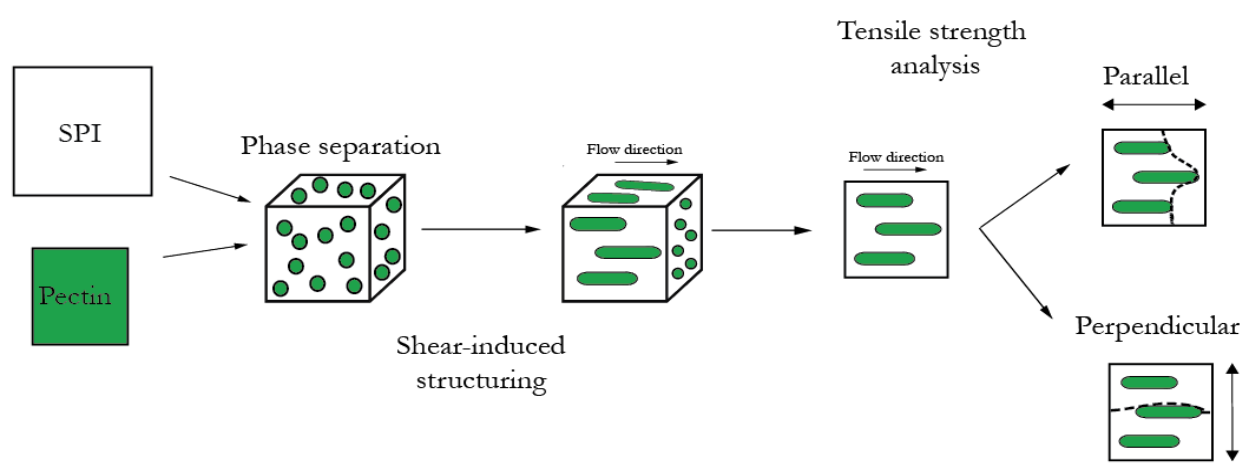

Fig. 4.6 Graphical illustration of shear-induced structuring, microstructure and fracture behaviour of pectin/soy protein isolate (SPI) blends that are deformed in parallel and perpendicular direction for anisotropic structures. 
of the pectin filament. The size and orientation of the pectin droplet can then be used to explain differences in fracture behaviour when deforming the structures parallel or perpendicular to the shear flow and hence the measured anisotropy. An elongated filament has more surface area in the perpendicular direction than in parallel direction, which explains a sharper decrease in tensile stress and strain-values.

We found that the outcomes of tensile strength analysis were dependent on pectin concentration and heating temperature. Higher concentrations of pectin gave more pronounced anisotropy in macrostructure, microstructure and mechanical properties compared to low concentrations of pectin $(1.3 \mathrm{wt} . \%)$. For low concentrations of pectin, both the microstructure and the anisotropic index were similar to a single component system, which is explained by co-solubility of SPI and pectin. Higher concentration of pectin (2.2 - $3.1 \mathrm{wt} . \%)$ ensures sufficient de-mixing of the two phases, leading to clear anisotropy in the tensile test and the microstructures. However, for high pectin concentrations $(4.0 \mathrm{wt} . \%)$, samples became very weak. Then, the width of the pectin filaments might become sufficiently large to also weaken the structure in both the parallel and perpendicular direction. A kind of phase inversion might have occurred, making the system bicontinuous or the weaker pectin phase continuous. Hence, not only the length of the pectin filaments but also the width, and therefore the volume or total surface area of the filaments play an important role for the ability to create anisotropy and to be able to measure it with a tensile strength analysis. This model system consisting of a soluble polysaccharide in a protein phase, i.e. pectin in SPI, resulted in fibrous structures.

Temperature influenced shear-induced structure formation of pectin/SPI blends, both on macroscopic and microscopic scale. A temperature effect was, however, hardly seen for structuring of SPI. In literature, SPI is reported to be rather stable at temperatures between 120 - $140{ }^{\circ} \mathrm{C}$ (Kinsella, 1979). The effect that temperature has on structure formation is mainly and effect of changes in the pectin and/or the interaction between pectin and SPI in the blends. In case of low temperature processing, many small droplets/filaments were formed that weakened the structure both in parallel and perpendicular direction, whereas the few large and elongated filaments formed at $140{ }^{\circ} \mathrm{C}$ resulted in mainly weakening of the structure in perpendicular direction. The number, length and width of the pectin filaments were, therefore, shown to be an important microstructural property that is related to anisotropy as measured with a tensile test. 
The effect that temperature has on pectin and/or the interaction between pectin and SPI can be related to non-enzymatic degradation of pectin. According to literature, non-enzymatic degradation of pectin, due to $\beta$-elimination, is accelerated with increasing temperatures at a $\mathrm{pH}$ above 5.5 (Diaz et al., 2007). The formation of shorter pectin chains, i.e. lower molecular weight, might result in lower viscosity of the dispersed phase (Chen et al., 2012; Diaz et al., 2007). Changes in ratio of viscosities of the dispersed, and continuous phase was already shown by early work of Taylor (1934) to influence droplet deformation in a given flow field (Taylor, 1932, 1934). Besides, non-enzymatic degradation of pectin can result in more reducing sugars in solution, and hence more Maillard reaction in the protein phase. In this way the SPI phase can be altered, but the resulting Maillard products can also induce more interaction between in the microstructure between the pectin and SPI phases. More interactions at the interface can result in better adhesion between the two phases, and hence higher tensile strengths for both the parallel and perpendicular directions, found at $140{ }^{\circ} \mathrm{C}$.

This study shows that fiber formation in a SPI-pectin blend is caused by the fact that pectin forms weaker and elongated filaments in the blend. We think that this effect also plays a role when structuring soy protein concentrate, either by shear-induced structuring or extrusion. Soy protein concentrate is a raw material that contains both protein and soluble polysaccharides (pectin) naturally. However, it also contains a insoluble carbohydrate (Day, 2013; Karr-Lilienthal et al., 2005). Further studies should therefore focus on understanding the role of that component as well. Alternatively, novel soy protein concentrates can be developed, with main focus on meat alternatives. In this study, it was shown that the ratio between protein and polysaccharides is an important parameter for fibrous structure formation, and the knowledge presented in this study enables a better design of fractionation process for soy flour.

\subsection{Conclusion}

Fibrous samples were obtained by shear-induced structuring of a pectin/SPI blend. SEM analysis revealed that shear-induced structuring resulted in elongated filaments of pectin, oriented in the direction of the shear flow entrapped in a continuous protein phase. The degree of elongation of these filaments was shown to play a key role in the degree of anisotropy as measured with the tensile strength analysis, and can also be related to the differences observed in the macrostructure. Anisotropy is dependent on the heating temperature and the ratio of the two biopolymers used. Layered structures correspond to a microstructure with relatively small elongated droplets, whereas fibrous structures have a microstructure with more elongated 72 
droplets. These outcomes on micro- and macrostructure were related to the tensile strength analysis. It was concluded that the tensile strength was dependent on the phase arrangement of the biopolymers. We hypothesize that a weak dispersed phase with an anisotropic nature is a route for making fibrous structures from a biopolymer blend.

\section{Acknowledgements}

The authors wish to thank ISPT for financial support. We are also thankful to Tiny FranssenVerheijen for her help preparing the SEM images, Jarno Gieteling for technical support, and the technical development studio (Wageningen UR) for help with shearing equipment. We acknowledge Nick van Lanen for his contribution by performing experimental work. 


\section{Chapter 5}

\section{Understanding fiber formation in a concentrated soy protein isolate - pectin blend}

This chapter has been published as B.L. Dekkers, R. Hamoen, R.M. Boom, \& A.J. van der Goot (2018). Understanding fiber formation in a concentrated soy protein isolate - Pectin blend. Journal of Food Engineering, 222, 84-92. http://doi.org/10.1016/j.jfoodeng.2017.11.014 


\section{Abstract}

Concentrated blends of pectin in soy protein, subjected to simple shear flow while heated form solid, fibrous materials that are a basis for meat analogues. The commonly accepted rule of mixing' based on the cross-sectional area of a weak dispersed phase was used to predict mechanical anisotropy of the material. Experimentally, two different dispersed phases were observed in the continuous soy protein matrix; air and pectin. An optimum in shape anisotropy of the air and pectin droplets, and mechanical anisotropy was found for a shear rate of $39 \mathrm{~s}^{-1}$. At higher shear rates, air was expelled and break-up of pectin droplets was found, resulting in materials with hardly any mechanical anisotropy. There was discrepancy between the modelled and experimental data when using the same mass fraction and volume fraction of pectin, whereas the model fitted better when assuming that the pectin phase absorbs more water relatively to the soy phase. 


\subsection{Introduction}

Solutions of biopolymers tend to phase-separate at sufficiently high concentration, leading to water-in-water (w/w) emulsions (Wolf, 2013). Water-in-water emulsions have been studied under static conditions (Ako, Durand, \& Nicolai, 2011; Firoozmand \& Rousseau, 2014; Norton \& Frith, 2001) and under deformation (Norton \& Frith, 2001; Simeone, Sibillo, Tassieri, \& Guido, 2002; Wolf, Scirocco, Frith, \& Norton, 2000). A distinguishing feature of w/w emulsions, is that the interfacial tension between the phases is generally very low (between $10^{-7}$ to $10^{-5}$ $\mathrm{N} / \mathrm{m}$ ), and therefore such a phase is more easily deformed than air bubbles or oil droplets, which are characterized by an appreciable interfacial tension (Erni, Cramer, Marti, Windhab, \& Fischer, 2009; Scholten, Tuinier, Tromp, \& Lekkerkerker, 2002; Simeone, Alfani, \& Guido, 2004; Walstra, 2004). The interfacial tension tends to keep the droplets spherical, and counteracts a deformation force, such as a force originating from a shear flow field. The relatively low resistance against deformation of $\mathrm{w} / \mathrm{w}$ emulsions makes them less susceptible to droplet breakup, and thus the dispersed phase tends to deform into elongated droplets or even long threads, which makes these systems suitable for the formation of anisotropic structures upon applying flow conditions.

Many food materials are multiphase systems. For example, foods may consist of a continuous phase containing a dispersed phase such as air bubbles, while the continuous phase itself is a w/w emulsion as well. Deformation of these types of multiphase systems can form several anisotropic structures, such as fibres, capillary filled gels, and lamellar structures after solidification by heating or crosslinking (Tolstoguzov, Mzhel'sky, \& Gulov, 1974; Wolf et al., 2000; Wolf \& Frith, 2003). The microstructure of these systems is dependent on the interaction between the dispersed phase in terms of droplet coalescence, deformation and breakup during flow (Grace, 1982; Guido, Simeone, \& Alfani, 2002; Tolstoguzov, 1991; Wolf et al., 2000). Droplet break up depends on the ratio between external, viscous and internal or interfacial forces (Taylor, 1934), which are related in the capillary number $(C a)$

$$
C a=\frac{\dot{\gamma} \eta_{c} D}{2 \sigma}
$$

in which $\dot{\gamma}$ is shear rate, $\eta_{c}$ is the viscosity of the continuous phase, $D$ is the droplet size, and $\sigma$ is the interfacial tension. A prerequisite for deformation is that the calculated $C a$-number is well below the critical $C a$-number. 
We earlier investigated shear-induced deformation of a concentrated pectin - soy protein isolate (SPI) blend (Dekkers, Nikiforidis, \& Van der Goot, 2016). SPI/pectin blends have been studied by others, mostly in lower concentrations to study complex coacervation (Lam, Paulsen, \& Corredig, 2008; Luisa, Freitas, Albano, Regina, \& Telis, 2017). At high concentration, mixing of the biopolymers is less likely to occur. Therefore this SPI/pectin blend formed a w/w emulsion when the $\mathrm{pH}$ of the blend was not changed. The pectin domains were deformed upon steady shear flow while heating at $140{ }^{\circ} \mathrm{C}$. After cooling to room temperature, this SPI/pectin blend had a fibrous meat-like appearance. The mechanical properties of the SPI/pectin blend were explained by considering the samples as gels with filled capillaries; a weak dispersed phase, which is deformed and entrapped in a stronger continuous phase. In the previous study, we hypothesized that the cohesiveness of the pectin phase or the adhesion between the pectin and SPI phase is weaker than the cohesiveness of the SPI phase itself. When the material is stretched during a tensile test, it is likely that detachment then occurs through or along the dispersed pectin droplets. This means in practice that fibres only appear upon tearing the product perpendicular to the shear flow. Recent findings showed that besides pectin also some air is entrapped in the structures and this might also form weak spots, along the same mechanism. It is hypothesized that both the dispersed pectin and the air bubbles will result in weakening of the overall structure.

In this study, we investigate the relation between shear-induced deformation of a concentrated SPI-pectin blend, its microstructure and the mechanical anisotropy of the material after solidification. A modified rule of mixing was used to predict the relation between the deformation of a weak dispersed phase in a stronger continuous phase, and the resulting mechanical anisotropy. Anisotropy development was studied in a SPI/pectin blend while varying the shear rate. The entrapment of air in samples was studied with X-ray tomography. Deformation of the dispersed pectin phase in the continuous SPI phase, and the overall microstructure were studied with scanning electron microscopy (SEM). The macro-mechanical properties of the material were determined with a tensile test. Mechanical anisotropy of the samples was determined by cutting tensile bars parallel and perpendicular to the shear flow. The difference between the tensile stress/strain parallel and perpendicular was used to compare the various samples. 


\subsection{Material \& Methods}

\subsubsection{Materials}

Soy protein isolate (SUPRO® 500E IP) was obtained from Solae (St Louis, MO). The isolate contained at least $83.4 \mathrm{wt} . \%$ protein ( $\mathrm{N}$ x 5.71). Pectin from citrus peel (P9135), sodium chloride (S9625), and glutaraldehyde (25 v.\%) were all obtained from Sigma-Aldrich (Germany). Ethanol (99\% purity) and acetone (99\% purity) were purchased from Actu-All Chemicals (Oss, The Netherlands).

\subsubsection{Shear-induced deformation}

Blends of pectin in SPI were studied by combining $2.2 \mathrm{wt} . \%$ pectin, $41.8 \mathrm{wt} . \%$ SPI, $1.0 \mathrm{wt} . \%$ salt and $55.0 \mathrm{wt} . \%$ demineralized water. The salt was first dissolved in the water. SPI was then added and mixed with a spatula. The protein blend was hydrated for $30 \mathrm{~min}$ in the water forming a crumbly dispersion. Subsequently pectin was mixed manually using a spatula through the protein dispersion; again, a few min were allowed for hydration of the pectin. The $\mathrm{pH}$ of this blend was approximately 6 . The blend was transferred into a preheated $\left(140^{\circ} \mathrm{C}\right)$ high temperature shear cell. The design of this shear cell is based on previously reported equipment (Grabowska et al., 2016; Habeych et al., 2008; Manski et al., 2008; Peighambardoust et al., 2008), and is a cone-cone device that creates steady shear flow by rotating the bottom cone. The device can be heated and cooled while shearing, using hot or cold thermal oil. Here, the thermal oil was used for heating to $140{ }^{\circ} \mathrm{C}$ during shear-induced deformation, which was shown to be the optimum temperature for creating anisotropy (Dekkers et al., 2016). The oil also allowed for fast cooling of the samples; the temperature inside the high temperature shear cell was reduced from $140{ }^{\circ} \mathrm{C}$ to $40{ }^{\circ} \mathrm{C}$ in five min. The shear rate $\left(0,7,13,26,39,65,130 \mathrm{~s}^{-1}\right)$ was applied with a Haake drive (Haake Polylab QC, Germany). During cooling, the cone was stationary. After cooling, the samples were solid and part of the samples were frozen at $-18{ }^{\circ} \mathrm{C}$ for analysis (SEM), and part of the samples were kept at room temperature for tensile testing.

\subsubsection{X-ray tomography}

For 3D non-invasively and non-destructively imaging, a X-ray tomographer with the brand name GE Phoenix v | tome |x m (General Electric, Wunstorf, Germany) was used. The system contains two X-ray sources. The $240 \mathrm{kV}$ micro focus tube with tungsten target was employed. X-rays were produced with a voltage of $80 \mathrm{kV}$ and a current of $90 \mu \mathrm{A}$. The images were recorded by a GE DXR detector array with $2024 \times 2024$ pixels (pixel size $200 \mu \mathrm{m}$ ). The detector was located 
$815 \mathrm{~mm}$ from the X-ray source. The samples $(3 \times 4 \times 20 \mathrm{~mm})$ were placed $28.55 \mathrm{~mm}$ from the $\mathrm{X}$-ray source. This result in a spatial resolution of $7.00 \mu \mathrm{m}$. A full scan consisted of 1500 projections over $360^{\circ}$, with a step ratio of $0.24^{\circ} \mathrm{C}$. The first image was skipped. The saved projection was the average of three images where every image is obtained over $250 \mathrm{~ms}$ exposure time. GE reconstruction software (Wunstorf, Germany) was used to calculate the 3D structure via back projection. The 3D images, obtained using the $\mathrm{v} \mid$ tome $\mid \mathrm{x}$ XRT, were analysed using Avizo imaging software version 9.2.0.

\subsubsection{Scanning Electron Microscopy}

Rectangles with a long edge (approximately $4 \mathrm{~mm}$ ) and a short edge $(2.5 \mathrm{~mm}$ ) were cut from the solid samples after shear-induced deformation. The samples were fixated in a glutaraldehyde solution $(2.5 \mathrm{v} . \%)$, while gently rotating for $8 \mathrm{~h}$. Next, they were soaked in demineralized water overnight. Subsequently, two sample preparation methods were used. One set of samples was fractured after soaking in demineralized water. Then, both fractured and intact samples were immersed in a series of solutions of ethanol (10, 30, 50, 70, 90 and $100 \mathrm{v} . \%)$ for at least $2 \mathrm{~h}$ per solution. Prior to critical-point drying (CPD 300, Leica, Vienna, Austria), ethanol was replaced with acetone. The second set of samples was prepared by fracturing samples after critical-point drying. The samples were fractured into two directions, to observe the surfaces of the samples from different angles (parallel and perpendicular to the shear flow). Samples were attached onto sample holders and sputter coated with a $10 \mathrm{~nm}$ thick layer of wolfram (SCD 500, Leica, Vienna, Austria). The surfaces were analysed with a field emission scanning electron microscope (Magellan 400, FEI, Eindhoven, the Netherlands) at a working distance of 4-8 mm, with SE detection at $2 \mathrm{kV}$ and $6.3 \mathrm{pA}$.

\subsubsection{Droplet analysis}

Fracturing of samples after fixation resulted in more regular surfaces compared to fracturing samples after critical point drying. A more regular surface area of the samples made a quantitative analysis of the droplet size possible. Ten pictures per fractured samples were used with a horizontal field width of $1.19 \mathrm{~mm}$. The minimal size of a droplet that could be measured in this way was $10 \mu \mathrm{m}$. The number of droplets, the length (a) and width (b) of the droplets parallel (1) and perpendicular (2) to the shear flow were measured with ImageJ software. From the droplet size distribution, characteristic droplet sizes $d_{0.1}, d_{0.5}$ and $d_{0.9}$ were determined. The $d_{0.5}$ refers to 
the median, $d_{0.9}$ refers to the droplet size where 90 percent of the droplets has a smaller droplet size and $d_{0.1}$ is the droplet size where 10 percent of the sizes of the droplets below that value.

\subsubsection{Mechanical properties}

The anisotropy of solid samples after shear-induced deformation was determined by cutting tensile bars (length $25 \mathrm{~mm}$, width $4.4 \mathrm{~mm}$ ) parallel and perpendicular (vorticity direction) to the shear flow; three samples were made per condition, and from each samples three tensile bars were taken in parallel and perpendicular direction. A texture analyser (INSTRON 5564, USA) was used to deform the samples with a constant deformation rate of $1 \mathrm{~mm} / \mathrm{s}$ approximately 1 hour after shear-induced deformation of the w/w emulsion to ensure that the samples are fully cooled down to room temperature. Samples were placed between two sand-coated clamps at a distance of $15.5 \mathrm{~mm}$. The tensile stress (force measured at rupture / area) and tensile strain (rupture extension / extension $)_{0}$ ) were determined from the stress-strain curve. In these calculations a correction was made for the differences in the thickness of the samples (i.e. the velocity gradient). Furthermore, the differences in tensile stress and tensile strain was determined for samples oriented in parallel and perpendicular direction.

\subsection{Theory}

Simple shear flow can be used to deform and align the SPI/pectin blend. Deformation of the dispersed phase, being droplets or air bubbles, results in an increase of the length of the droplet or bubble in the direction of the flow, whereas the width decreases since the volume of the dispersed phase should remain constant. We will simplify deformation of the blend by considering the dispersed phase as many small cubes inside a larger cube, which represents the continuous phase. The volume of such a small cube $\left(V_{D}\right)$ can be calculated with

$$
V_{D}=\frac{\phi_{D, t o t}}{n}
$$

with $\phi_{D, t o t}$ being the volume fraction of the dispersed phase, and $n$ being the total number of cubes. We assume that all these cubes have a similar size and deform in a similar manner. The volume $V$ of a domain is given by its length, width and breadth $(l, w$ and $b$, respectively)

$$
l \cdot w \cdot b=V_{D}
$$

Deformation leads to one dimension becoming longer (e.g., $l$ ), and if we assume that the volume remains constant, and the two other dimensions will remain the same, 


$$
w=b=\sqrt{\frac{V_{D}}{l}}
$$

in which $l$ is the size of the cube in the shear flow direction, and $w$ and $b$ are the sizes in the other directions i.e. the vorticity and velocity gradient direction. Deformation of the droplets under flow can then be quantified by calculating the ratio between the long side $(l)$ of a droplet and the width $(w)$ of a droplet, $l / w$. Deformation of the dispersed phase results in mechanical anisotropy when the dispersed phase has a different strength compared to the continuous phase. Here, we consider mechanical anisotropy arising from a weak deformed dispersed phase, or the adhesion between the continuous and dispersed phases is weak. Mechanical anisotropy, in a tensile test when stretching the material parallel and perpendicular to the shear flow, is correlated with the difference in the cross-sectional surface area of the weak dispersed phase parallel and perpendicular to the shear flow or inversely the cross-sectional surface area of the strong continuous phase parallel and perpendicular to the shear flow.

Several models were developed to predict the mechanical properties of composite materials (Ahmed \& Jones, 1990; Facca, Kortschot, \& Yan, 2006; Morley, 1976). A simple model for the estimation of the strength of a composite, referred to as the rule of mixing (ROM), assumes the geometry to be one of two extremes; either a stack of plates parallel to the direction of the stress, or a stack of plates perpendicular to the stress. In the latter case, the stress on the two phases is the same, and the overall composite stress $\sigma_{C \text {,parallel }}$ is then given by a volumetric average (Ahmed \& Jones, 1990). In the former case with a parallel stacking, the strain $\epsilon$ will be uniform over the two phases, and the tensile strength of the composite material $\sigma_{0}$ can then be calculated with $(\sigma=E \epsilon)$ :

$$
\begin{gathered}
E_{0}=\phi_{d} E_{d}+\phi_{c} E_{c} \rightarrow E_{0} \epsilon=\phi_{d} E_{d} \epsilon+\phi_{c} E_{c} \epsilon \rightarrow \\
\sigma_{o}=\phi_{c} \sigma_{c}+\phi_{d} \sigma_{d}=\sigma_{c}-\phi_{d}\left(\sigma_{c}-\sigma_{d}\right)
\end{gathered}
$$

where $\sigma_{o}, \sigma_{c}$, and $\sigma_{d}$ are the tensile stresses of the overall material, continuous, and dispersed phase, and $\phi_{d}$ and $\phi_{c}$ are the volume fractions of the continuous and dispersed phase.

We use equation 4 to describe the maximum stress that the material can have, over a crosssection that contains the dispersed phase: this particular cross-section will behave according to the model that has plates parallel to the direction of the stress. The typical surface fraction of the dispersed phase parallel $\left(\alpha_{d \|}\right)$ to the direction of the stress, is given by 


$$
\alpha_{d \|}=\frac{w b}{(w b l)^{2 / 3}} \phi_{d}=\frac{w^{2}}{\left(w^{2} l\right)^{2 / 3}} \phi_{d}=\left(\frac{l}{w}\right)^{-2 / 3} \phi_{d}
$$

with $l / w$ being the index of deformation, and $l$ the dimension in the direction of the shear flow, with $l>w$. This yields with equation 4 and 5 for the maximum stress along the direction of the stress $\left(\sigma_{o \|}\right)$ :

$$
\sigma_{o \|}=\sigma_{c}-\left(\frac{l}{w}\right)^{-2 / 3} \phi_{d}\left(\sigma_{c}-\sigma_{d}\right)
$$

The surface fraction perpendicular to the direction of the stress $\left(\alpha_{d \perp}\right)$, is given by

$$
\alpha_{d \perp}=\frac{w l}{\left(w^{2} l\right)^{2 / 3}} \phi_{d}=\left(\frac{l}{w}\right)^{1 / 3} \phi_{d}
$$

Combination of equation 4 and 7, the maximum stress perpendicular to the direction of the stress is given by $\left(\sigma_{o \perp}\right)$ :

$$
\sigma_{o \perp}=\sigma_{c}-\left(\frac{l}{w}\right)^{1 / 3} \phi_{d}\left(\sigma_{c}-\sigma_{d}\right)
$$

When the droplets are more deformed, and thus exhibit a larger ratio $l / w$, the material becomes stronger in parallel direction and weaker in the perpendicular direction. In Fig. 5.1A, the parallel and perpendicular tensile stress are plotted for various dispersed phase tensile stresses $\left(\sigma_{d}=\right.$ $200,100,1 \mathrm{kPa})$, while keeping a constant continuous phase tensile stress $\left(\sigma_{c}=600 \mathrm{kPa}\right)$ and a constant dispersed phase fraction $\left(\phi_{d}=0.1\right)$. The absolute values differ mainly in perpendicular direction. This results in a larger difference in stress in parallel and perpendicular direction for a weaker dispersed phase. In Fig. 5.1B the prediction of tensile stress/strain is plotted for various dispersed phase volume fraction $\left(\phi_{d}=0.2,0.01,0.05\right)$, while keeping tensile stress of the continuous and dispersed phase constant $\left(\sigma_{c}=600 \mathrm{kPa}\right.$ and $\left.\sigma_{d}=100 \mathrm{kPa}\right)$. The dispersed volume fraction has an effect on both the tensile stress parallel and perpendicular to the shear flow. The dotted line in both figures indicates the maximum value of the tensile stress of the continuous phase, in this case SPI, which has a tensile stress of roughly $600 \mathrm{kPa}$ (Dekkers et al., 2016). 

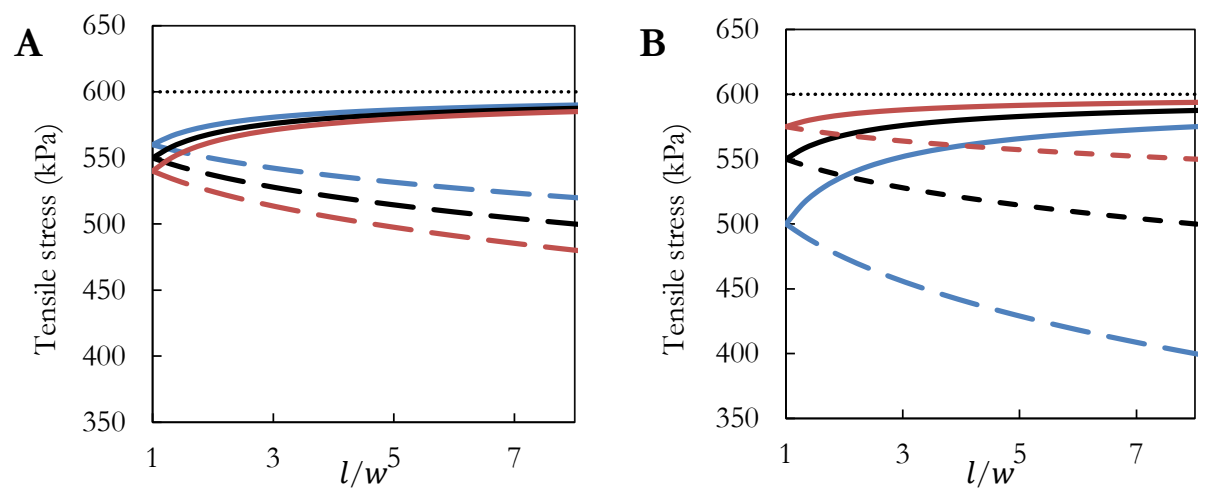

Fig. 5.1 Prediction of tensile stress parallel (solid line) and perpendicular (dashed line) to the shear flow, for A) $\sigma_{c}=$ 600, $\sigma_{d}=200$ (blue), $\sigma_{c}=600, \sigma_{d}=100$ (black) and $\sigma_{c}=600, \sigma_{d}=1$ (red) with similar $V_{D, \text { tot }}=0.1$ and B) $V_{D, \text { tot }}=0.05$ (red), $V_{D, \text { tot }}=0.1$ (black), and $V_{D, \text { tot }}=0.2$ (blue) and $\sigma_{c}=600, \sigma_{d}=100$. The dotted line is the tensile stress of the continuous phase alone.

\subsection{Results and discussion}

Shear-induced deformation while heating a concentrated soy protein isolate (SPI) - pectin blend was studied for a better understanding of the formation of anisotropy. The blend, containing pectin and SPI and also some air bubbles, was deformed by applying steady shear flow. During deformation, the material was heated to $140{ }^{\circ} \mathrm{C}$, and subsequent fast cooling ( $5 \mathrm{~min}$ ) without deformation resulted in solidification of the material. We mirrored the experimental data with the predictions of the model from the theoretical section by quantifying shape and mechanical anisotropy with X-ray tomography, scanning electron microscopy and tensile testing. The shear rate was varied to study various degrees of deformation of the dispersed phases. We observed that the most pronounced fibrous structures were obtained when we used moderate shear rates $\left(26-130 \mathrm{~s}^{-1}\right)$.

\subsubsection{Entrapment and deformation of air}

X-ray tomography was used to describe how entrapped air was distributed in the samples during shear-induced deformation. This technique is based on the detection differences in material density. Since air has a different density than the two biopolymers, we can distinguish the air phase, whereas SPI and pectin in water have similar density and thus cannot be distinguished with this method. SPI alone yielded a layered structure with 2.5 v.\% air entrapped when sheared at $39 \mathrm{~s}^{-1}$. At the same shear rate, addition of pectin resulted in fibrous structures with $8.4 \mathrm{v} . \%$ air entrapped. Samples that were not sheared contained 9.5 v.\% air, irrespective of whether they 
contained pectin or not. It is thus clear that the presence of the pectin hinders the expelling of air bubbles from the matrix during shearing.

The two-dimensional X-ray tomography images were reconstructed to obtain a 3D structure (Fig. 5.2). In absence of a flow field, the air is present in spherical bubbles, and almost no orientation was observed (Fig. 5.2A). Shearing the SPI-pectin blend resulted in fibrous structures in which the air is also aligned in the shear flow direction. In these samples, there was hardly any difference observed in the air bubble ordering in the vorticity and velocity gradient. For the pure SPI samples, the air was also aligned in the shear flow direction (Fig. 5.2C), but the air was ordered in layers along the velocity gradient (the gap width of the shearing device).

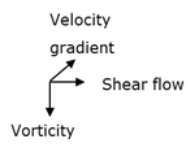

A
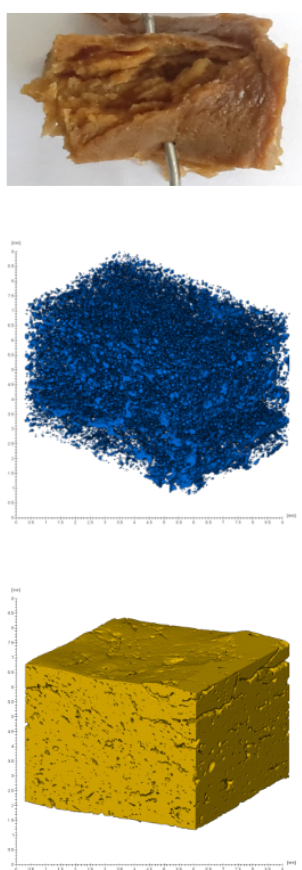

B
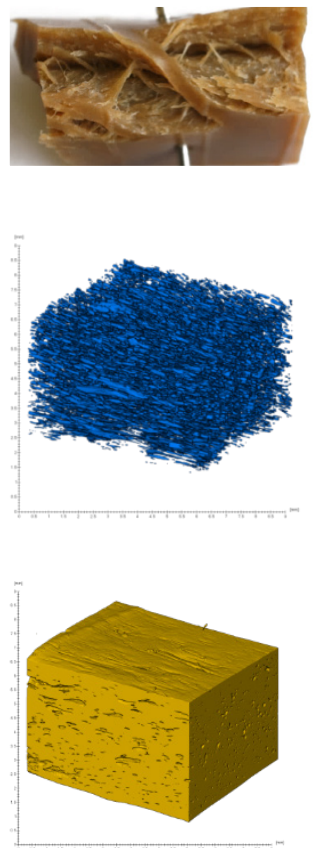

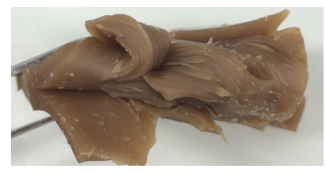

C
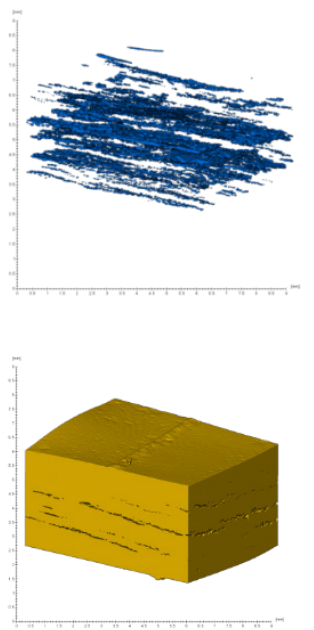

Fig. 5.2 Pictures and X-ray tomography images of $A$ ) non-sheared sample $\left(0 \mathrm{~s}^{-1}\right)$ consisting of $41.8 \mathrm{wt} . \%$ SPI and $2.2 \mathrm{wt} . \%$ pectin, B) sheared sample $\left(395^{-1}\right)$ ) consisting of $41.8 \mathrm{wt} . \%$ SPI and $2.2 \mathrm{wt. \%}$ pectin, and C) sheared sample $\left(39 \mathrm{~s}^{-1}\right)$ consisting of $44 \mathrm{wt} . \%$ SPI. Top: macrostructure, middle: a reconstructed trimetric image of the air in blue, and bottom: a reconstructed trimetric image of the solids in yellow.

The reconstructed 3D structures were used to determine: porosity, the length of the deformed air bubbles in the shear flow direction (defined as $l$ ) and the largest value of the diameter of such 
a bubble in the vorticity or velocity gradient direction (defined as $w$ ). From $l$ and $w$, the $l / w$ of the air bubbles was calculated.

Shear rate $\left(0-130 \mathrm{~s}^{-1}\right)$ affected the entrapment of air and its deformation (Fig. 5.3). Up to a shear rate of $39 \mathrm{~s}^{-1}$, the differences in porosity were rather small (variation between 8.4 - 10.5 v.\%). Higher shear rates resulted in strongly lower porosity of the samples; samples sheared at $65 \mathrm{~s}^{-1}$ contained $3.3 \mathrm{v} . \%$ air, and $130 \mathrm{~s}^{-1}$ contained 1.4 v.\% air only. Shear rate $\left(0-130 \mathrm{~s}^{-1}\right)$ also affected the $l$ and $w$ of the air bubbles. An increase in air bubble length was found when varying the shear rate between $0 \mathrm{~s}^{-1}$ and $39 \mathrm{~s}^{-1}(86 \rightarrow 200 \mu \mathrm{m})$, and at higher shear rates $\left(65\right.$ and $\left.130 \mathrm{~s}^{-1}\right)$ the length decreased again $(200 \rightarrow 72 \mu \mathrm{m})$. The width of air bubbles hardly changed as function of the shear rate $(33-61 \mu \mathrm{m})$. Similarly to the air bubble volume, the highest $l / w$ was therefore found for a shear rate of $39 \mathrm{~s}^{-1}$ (Fig. 5.3B). Since not all the air bubbles were perfectly aligned by the shear flow, we measured the angle of the long side of droplets with the shear flow direction and corrected for this in a volume weighted fashion. However, this did not change $l / w$ significantly.
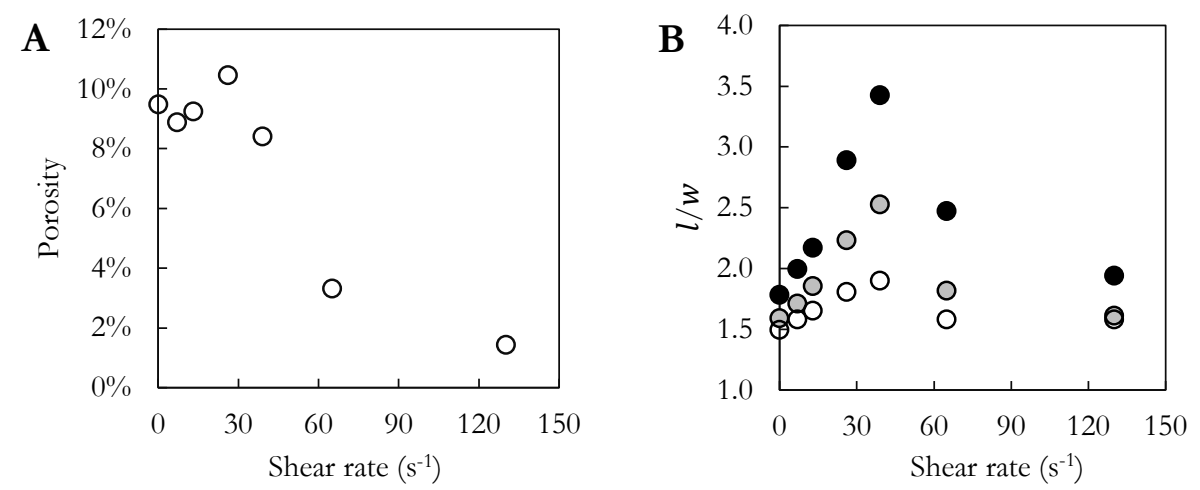

Fig. 5.3 X-ray tomography analysis of air bubbles when varying the shear rate, for a blend consisting of SPI and pectin: A) porosity, and B) length/width $(l / w)$ in quantiles; $d_{0.1}(\bigcirc), d_{0.5}(\odot)$, and $d_{0.9}$

\subsubsection{Deformation of the dispersed pectin phase}

$\mathrm{X}$-ray tomography showed that significantly more air was entrapped in the structures in SPIpectin blends as compared to SPI alone. Furthermore, earlier work showed that these conditions lead to fiber formation instead of layers. Since it was not possible to study the pectin phase with X-ray tomography, scanning electron micrographs (SEM) were made of the samples to study the microstructural changes in SPI-pectin blends under shear induced deformation. We estimated the deformation of pectin droplets by cutting the samples parallel and perpendicular to the shear flow. This yielded samples with a surface of the shear flow and velocity direction and surfaces 86 
of the velocity gradient and vorticity direction. We assumed that analysing a range of $2 \mathrm{D}$ images was sufficient to obtain a good impression of the $3 \mathrm{D}$ structure.

The samples were studied at several stages during the sample preparation procedure, since the surfaces of the microstructures were influenced by this fracturing step in the sample preparation. Samples that were fractured after critical point drying showed two domains with pectin forming dispersed droplets in a continuous phase of SPI (Fig. 5.4A), and were similar to the microstructures obtained in a previous study (Dekkers et al., 2016). These blends had a $\mathrm{pH}$ above the isoelectric point, and therefore both biopolymers have similar charge and repel each other. This is distinctly different from processing of SPI and pectin at $\mathrm{pH}$ around or below the isoelectric point at low concentrations, which results in complex coacervation (Lam, Shen, Paulsen, \& Corredig, 2007; Luisa et al., 2017). The continuous phase was dense, while the dispersed phase was porous after dehydration (shown in Fig. 5.4B). Samples that were fractured after fixation in glutaraldehyde (i.e. earlier in the sample preparation method) were softer, leading to larger fracture surface areas. In these samples a dense, particulate network was observed (Fig. 5.4C-D), with smooth gaps of similar size and shape as the pectin droplets, and we therefore assumed that these gaps represented the pectin droplets. The washing step after fixation probably resulted in dissolution and subsequent removal of the pectin. Some of these gaps may also originate from the air entrapped during shear-induced deformation.
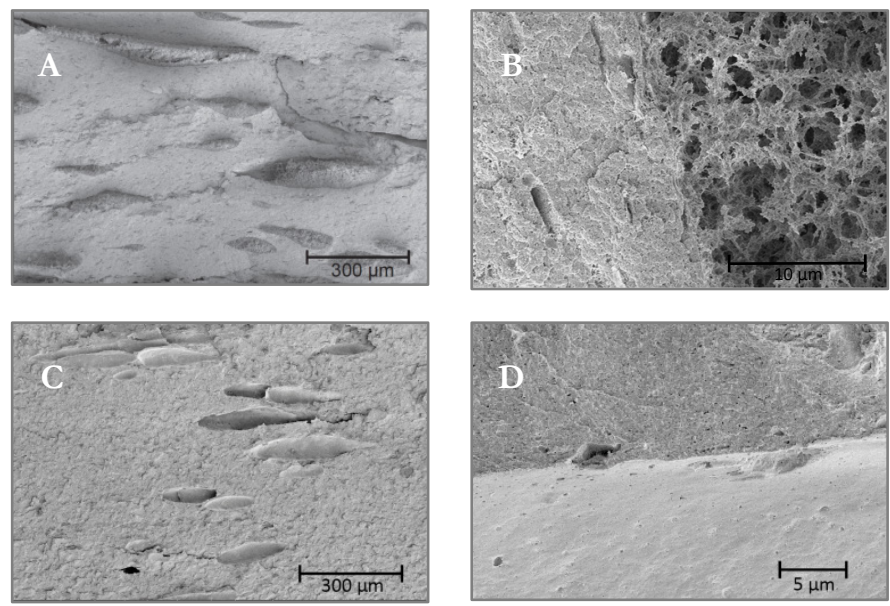

Fig. 5.4 Scanning electron micrographs of samples (sheared at $39 \mathrm{~s}^{-1}, 15$ min at $140{ }^{\circ} \mathrm{C}$ ) fractured after A) critical point drying, magnification 250 times B) critical point drying, magnification 10,000 times C) fixation in glutaraldehyde, magnification 250 times D) fixation in glutaraldehyde, magnification 10,000 times. 
The shear rate affected the deformation of the dispersed pectin droplets. In absence of a flow field (shear rate $0 \mathrm{~s}^{-1}$ ), the system featured spherical droplets (Fig. 5.5A). Shearing at $7 \mathrm{~s}^{-1}$ resulted in elongated droplets, with the surface droplet size slightly increased (Fig. 5.5B). Droplets sheared at $13 \mathrm{~s}^{-1}$ were larger and showed more deformation (Fig. 5.5C). Droplets sheared at 26 and $39 \mathrm{~s}^{-1}$ had similar sizes and were deformed similarly (therefore only those sheared at $39 \mathrm{~s}^{-1}$ are shown in Fig. 5.5D). At low magnification (250 times), no clear droplets were observed for higher shear rates $\left(65 \mathrm{~s}^{-1}\right.$, Fig. 5.5E-1), but higher magnifications did show small droplets, which were smaller than $10 \mu \mathrm{m}$ (Fig. 5.5E-2). It is clear that shear rate had an influence on the external forces that are resulting in deformation and breakup of droplets.
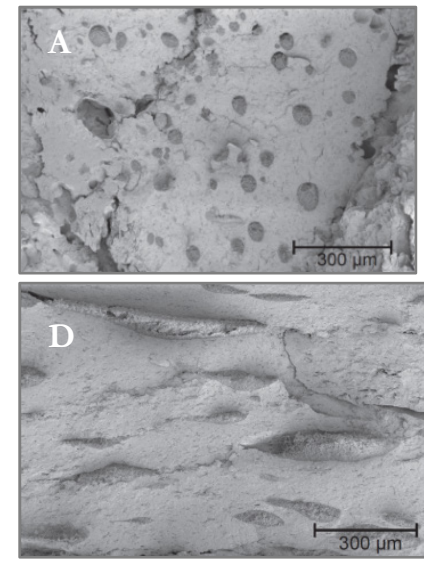
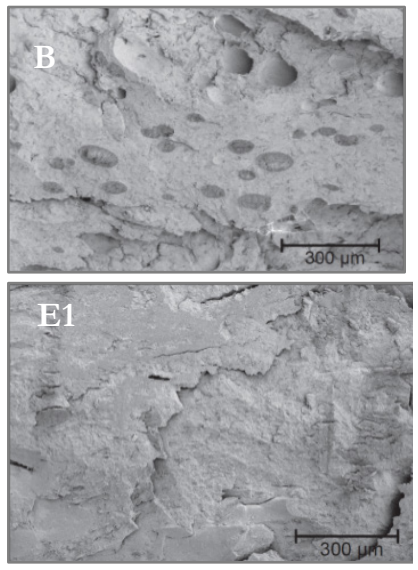
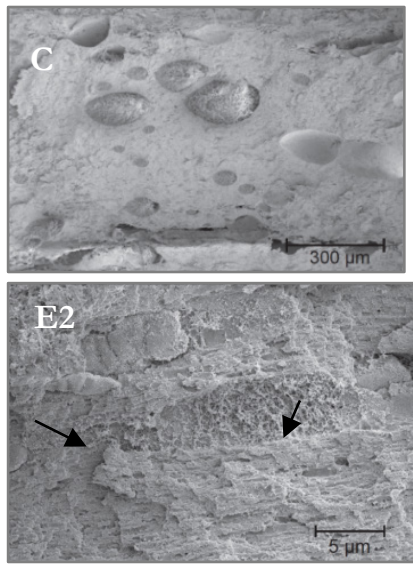

Fig. 5.5 Scanning electron micrographs of fractured samples that were sheared for various shearing rates for $15 \mathrm{~min} A) 0 \mathrm{~s}^{-1}$, B) $7 s^{-1}$, C) $13 s^{-1}$, D) $39 s^{-1}$ E) $65 s^{-1}$ at a magnification of 250 times (1) and magnification of 10,000 times in which dispersed pectin in the continuous SPI phase is indicated with black arrows (2).

The number, size and deformation of the pectin droplets were analysed for blends that were fractured after fixation. We measured the droplet size from ten scanning electron micrographs (horizontal field width of $1.19 \mathrm{~mm}$ ). Fig. 5.6 shows the $d_{0.1}, d_{0,5}$ and $d_{0.9}$ of the length and width of droplets parallel to the shear flow. The $l / w$ (equation 7 and 9 ) was used to describe shape anisotropy (in a $2 \mathrm{D}$ plane). Also scanning electron micrographs were made from samples perpendicular to the shear flow. In this direction, the droplets turned out to be spherical, having a size similar to the width of the droplets in the parallel direction.

Under static conditions, many spherical droplets were observed with an average diameter of approximately $35 \mu \mathrm{m}$. This droplet size was slightly larger than sizes $(2-20 \mu \mathrm{m})$ commonly found in literature for dilute to semi-dilute concentrated w/w emulsions after phase separation in absence of a flow field (Norton \& Frith, 2001). Here, the pectin and SPI were not mixed very 88 
intensive from the start, leading to larger droplets and a distribution in droplet sizes. The number of droplets measured from ten images decreased with increasing shear rates. Furthermore, three regimes in droplet sizes could be distinguished after shear induced deformation. Low shear rates $\left(7 \mathrm{~s}^{-1}\right)$ resulted in slight deformation and relatively small differences between $d_{0.1}$ and $d_{0.9}$. Intermediate shear rates $\left(13-39 s^{-1}\right)$ resulted in only small differences in the length of droplets, and the differences between $d_{0.1}$ and $d_{0.9}$ became larger indicating that the droplet size distribution became broader. High shear rates $\left(>65 \mathrm{~s}^{-1}\right)$ resulted in droplet breakup and therefore the dispersed droplets became smaller, less deformed and hence difficult to measure with the applied method. The average length of droplets increased significantly $(34 \rightarrow 145 \mu \mathrm{m})$, whereas the width of droplets $(42 \rightarrow 52 \mu \mathrm{m})$ increased hardly.
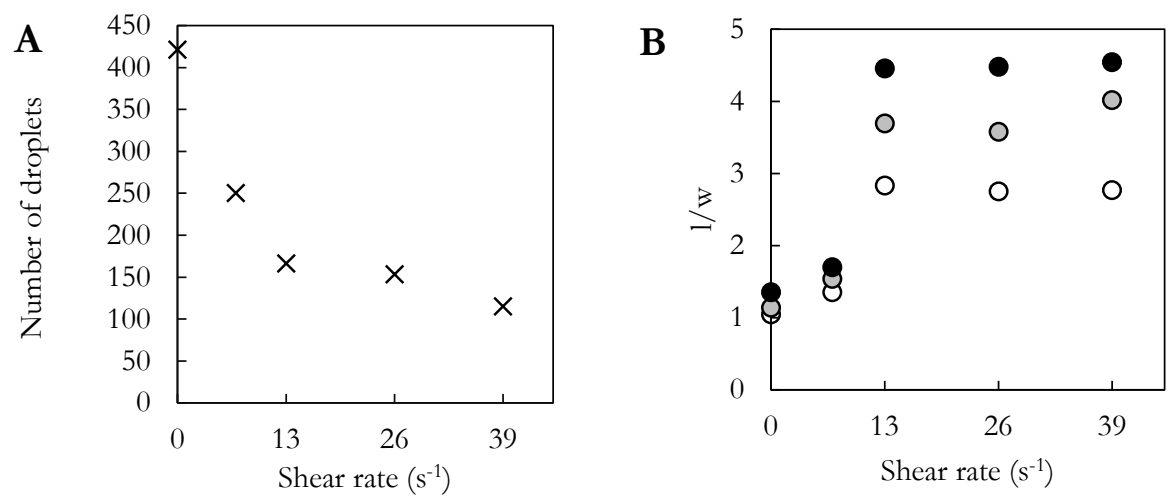

Fig. 5.6 Analysis of dispersed pectin droplets parallel to the shear flow when varying the shear rate A) number of droplets $(\times), B)$ length/width $(l / w)$ in quantiles; $d_{0.1}(O), d_{0.5}(\odot)$, and $d_{0.9}$

The number of pectin droplets counted in ten scanning electron micrographs decreased with increasing shear rate $\left(0-39 \mathrm{~s}^{-1}\right)$ and the absolute length and width of the pectin droplets increased (data not shown). Therefore it is likely that droplet coalesce had occurred, resulting in larger droplets. Fig. 5.7 shows a micrograph in which this droplet coalescence is observed. The lower numbers of pectin droplets at higher shear rates indicates that the coalescence rate is higher for increasing shear rate (up to $39 \mathrm{~s}^{-1}$ ). The number of droplets decreased more dramatically between $0-13 \mathrm{~s}^{-1}$ and levelled off between to $13-39 \mathrm{~s}^{-1}$.

Wolf et al. (2000) showed that droplets coalesce faster at low shear rates (Wolf et al., 2000). Furthermore, Emin et al. (2012) found that the coalescence intensity increased with increasing shear rates in the shear cell (Emin, Schmidt, van der Goot, \& Schuchmann, 2012), which might be explained by a lower matrix viscosity due to the shear thinning behaviour of SPI (Bengoechea, Arrachid, Guerrero, Hill, \& Mitchell, 2007). Wildes et al. (1999) suggested that a lower matrix 
viscosity facilitates film drainage and hence increases the rate of particles coalescing (Wildes, Keskkula, \& Paul, 1999).
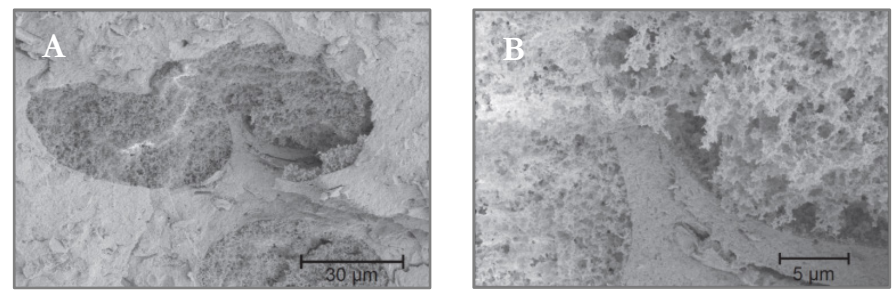

Fig. 5.7 Droplet coalescence: A) magnification 250 times, B) magnification 10,000 times

In summary, our conclusion from the SEM imaging is, that at intermediate shear rates, the pectin droplets become strongly elongated, but at higher shear rates, they break up into small droplets, making the number of visible droplets much smaller.

\subsubsection{Mechanical anisotropy}

The mechanical properties of the solid material after shear-induced deformation were determined with a tensile test. The tensile stress and strain at rupture showed an optimum for samples sheared at $39 \mathrm{~s}^{-1}$ (Fig. 5.8). In absence of a flow field (i.e. only heated for $15 \mathrm{~min}$ ), the samples were weak and as expected had similar properties parallel and perpendicular to the shear flow. Samples sheared at 7 and $13 \mathrm{~s}^{-1}$ resulted in slight anisotropy. These samples had a higher maximum tensile stress parallel to the shear flow, and lower tensile stress perpendicular to the shear flow when compared to samples that were not sheared. Samples sheared at 26 and $39 \mathrm{~s}^{-1}$ were highly anisotropic, and these samples had an even higher tensile stress in the parallel direction, but also a slight increase was found in the perpendicular direction. The increased tensile stress and strain in both directions at higher shear rates can be the result of lower porosity/air entrapment. As shown in the theoretical section, when the volume fraction of the weak dispersed phase becomes lower, then the tensile stress of the perpendicular direction shifts up. An optimum in tensile stress and strain difference was found for a shear rate of $39 \mathrm{~s}^{-1}$. The tensile stress and strain of samples sheared at $39 \mathrm{~s}^{-1}$ in the parallel direction was similar to an isotropic SPI sample (44 wt.\%), suggesting almost no weakening effect in this direction (Dekkers et al., 2016). Isotropic behaviour (i.e. similar tensile stress and strain values) was found for samples sheared at 65 or $130 \mathrm{~s}^{-1}$. These samples had a maximum tensile stress and strain values similar non-sheared SPI-pectin samples $\left(0 \mathrm{~s}^{-1}\right)$. 

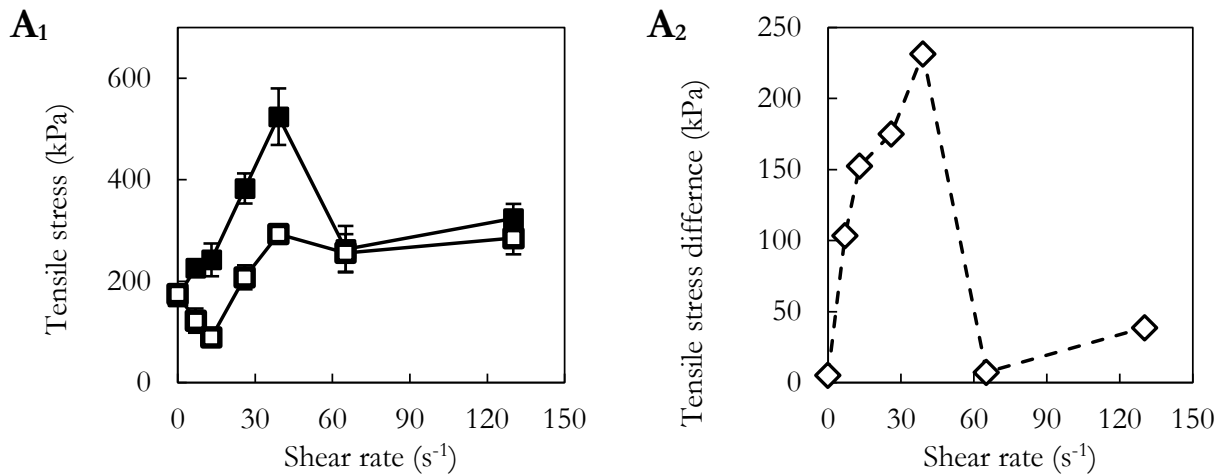

$\mathbf{B}_{1}$

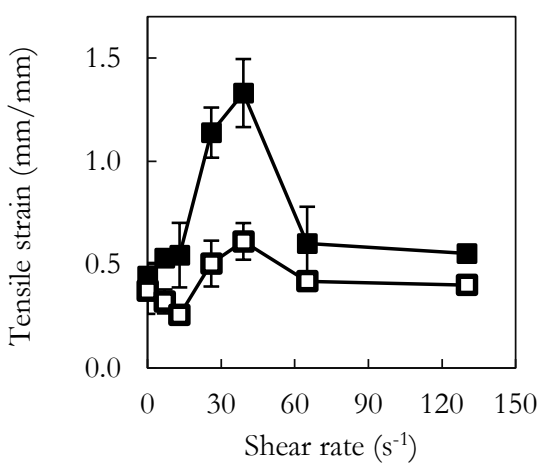

$\mathbf{B}_{2}$

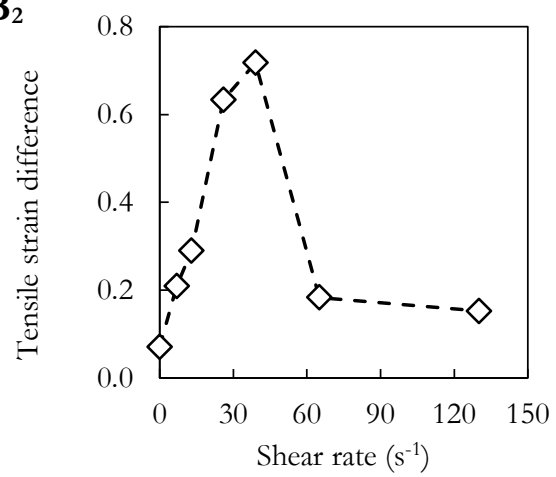

Fig. 5.8 Tensile stress (A), and tensile strain (B) with standard errors for samples that were sheared at various shear rates for 15 min, which are deformed in parallel (-) and perpendicular $(\mathbf{\square})$ direction (1), the corresponding difference between parallel and perpendicular $(\diamond)(2)$.

\subsubsection{Relation between shape \& mechanical anisotropy}

The tensile strength analysis showed that the mechanical anisotropy increased with increased shear rates and was optimal at a shear rate of $39 \mathrm{~s}^{-1}$. The XRT imaging showed that the deformation of the air bubbles increased with increased shear rates, whereas the porosity decreased with increasing shear rates. Similarly, the pectin droplets were shown to deform strongly up to a shear rate of $39 \mathrm{~s}^{-1}$. At higher shear rates $\left(65\right.$ and $\left.130 \mathrm{~s}^{-1}\right)$, the mechanical anisotropy disappeared, which may be related to break-up of the dispersed pectin phase, which also lead to less deformed droplets as well as the air being pushed out of the samples. Based on these findings, we propose three hypothesis; i) deformation of air results in mechanical anisotropy, ii) deformation of pectin results in mechanical anisotropy, or iii) deformation of air and pectin result in mechanical anisotropy. 
To verify these hypothesis, the $l / w$ of air with XRT and of pectin with SEM can be used to calculate the tensile stress in parallel $\left(\sigma_{o \perp}\right)$ and perpendicular $\left(\sigma_{o \|}\right)$ to the shear flow (equation 9 and 7). These equations can be simplified, when assuming $\sigma_{c} \gg \sigma_{d}$, to

$$
\begin{gathered}
\sigma_{o \|}=\sigma_{c}\left(1-\left(\frac{l}{w}\right)^{-\frac{2}{3}} \phi_{d}\right) \\
\sigma_{o \perp}=\sigma_{c}\left(1-\left(\frac{l}{w}\right)^{\frac{1}{3}} \phi_{d}\right)
\end{gathered}
$$

When air and pectin are both important for mechanical anisotropy (hypothesis iii), the following equations can be used

$$
\begin{gathered}
\sigma_{o \|}=\sigma_{c}\left(1-\left(\frac{l}{w}\right)^{-\frac{2}{3}} \phi_{d}\right)_{\text {air }} \cdot\left(1-\left(\frac{l}{w}\right)^{-\frac{2}{3}} \phi_{d}\right)_{\text {pectin }} \\
\sigma_{o \perp}=\sigma_{c}\left(1-\left(\frac{l}{w}\right)^{\frac{1}{3}} \phi_{d}\right)_{\text {air }} \cdot\left(1-\left(\frac{l}{w}\right)^{\frac{1}{3}} \phi_{d}\right)_{\text {pectin }}
\end{gathered}
$$

In these equations the $\sigma_{c}$ of $600 \mathrm{kPa}$ was used, which is the tensile stress of SPI sample. It must be noted that the porosity of SPI alone was $2.5 \mathrm{v} . \%$ and these air bubbles were also slightly deformed $(l / w=1.9)$. The deformed air bubbles in SPI might lower the tensile stress, and hence the $\sigma_{c}$ of the SPI phase without air might have been even higher. The porosity of the samples can be inserted for $\phi_{d, a i r}$. However, the $\phi_{d \text {,pectin }}$ should be estimated, since this was not measured directly from the SEM images.

In Fig. 5.9, two parity plots of the experimentally determined tensile stress difference and the predicted tensile stress difference are shown for the three hypothesis; one parity plot in which we assumed the $\phi_{d, p e c t i n}=0.05$, which was similar to the mass fraction, and in the second parity plot we assumed that pectin absorbed relatively more water with $\phi_{d, p e c t i n}=0.15$. It was shown that the prediction of the tensile stress differences based on the $l / w$ of air was not sufficient to fully explain the anisotropy, since the predicted tensile stress difference was much lower compared to the experimental tensile stress difference. When assuming that more water is absorbed by the pectin phase, then the experimental and predicted tensile stress differences became more similar. However, it is the question whether the volume fraction of pectin is this large. The volume fraction of pectin depends on the water distribution between SPI and pectin 
after shear-induced structuring. Previous research showed that when varying the ratio between SPI and pectin, the samples decreased in strength rapidly (Dekkers et al., 2016). According to the calculations done in the theoretical sections (Fig. 5.1B), this is possible when the pectin phase indeed absorbs more water.
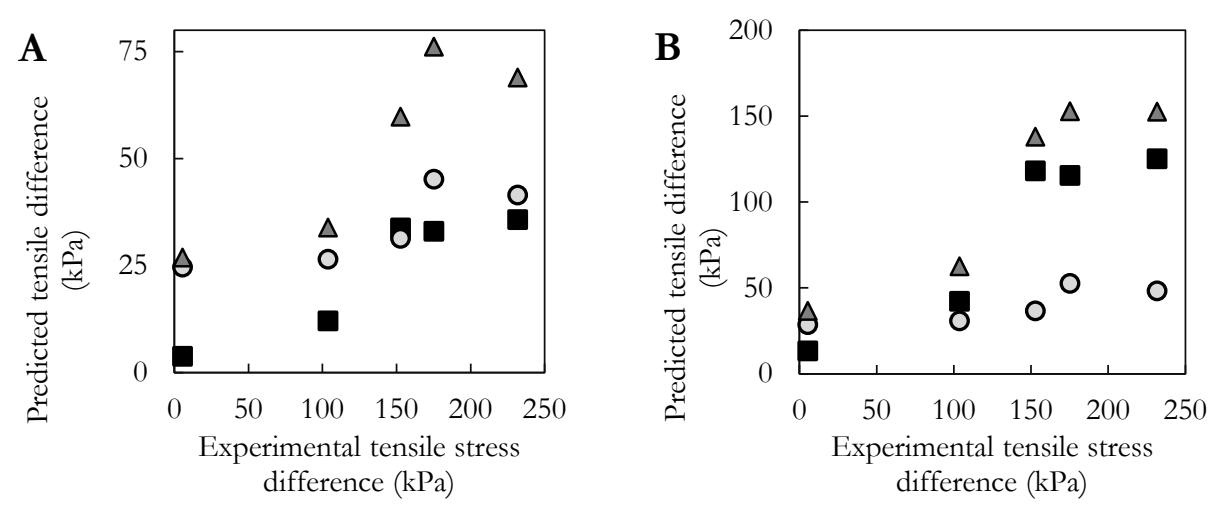

Fig. 5.9 Parity plots of experimental and predicted tensile stress based on the deformation $(l / w)$ of dispersed pectin $(\mathbf{\square})$, air (O), and air + pectin $(\mathbf{\Delta})$ when using $\sigma_{c}=600 \mathrm{kPa}, \phi_{d, \text { air }}=$ porosity, and A) $\phi_{d, p e c t i n}=0.05$ and B) $\phi_{d, \text { pectin }}=$ 0.15 .

\subsection{Conclusion}

Development of anisotropy in soy protein isolate (SPI) - pectin blends was investigated at high concentrations (44 wt.\%). In the theoretical section, shape anisotropy was related to mechanical anisotropy using the modified rule of mixing in which the cross-sectional area of the dispersed phase was used instead of the volume fraction. From this model, we found that mechanical anisotropy depends on four variables; the tensile stress or strain of the continuous phase, the tensile stress or strain of the dispersed phase, the volume fraction of the dispersed phase, and the deformation of the dispersed phase.

Experimentally, shape anisotropy was studied for two phases; entrapped air bubbles and dispersed pectin droplets. Shape anisotropy of both phases developed with varying shear rates; a higher shear rate resulted in increased droplet and bubble deformation up to a shear rate of 39 $\mathrm{s}^{-1}$. In addition to deformation of the dispersed phase, also coalescence of the dispersed phase plays an important role in shear-induced deformation processes. Air bubble coalescence was concluded from an increased average air bubble size when the porosity of the overall samples was roughly constant and was also observed for pectin droplets in the scanning electron micrographs. Coalescence resulted in the formation of larger droplets that become prone to 
elongation. At higher shear rates $\left(65-130 \mathrm{~s}^{-1}\right)$, pectin droplets were no longer observed, which indicates break-up of this weak dispersed phase into very small droplets. In the case of air, the porosity was lower and air was pushed out of the samples. The loss of shape anisotropy at these shear rates, is also related to the tensile stress/strain difference between parallel and perpendicular deformation of structures that disappeared at these shear rates.

\section{Acknowledgments}

The authors wish to thank Tiny Franssen-Verheijen for her help preparing the SEM images, Jarno Gieteling for technical support, the technical development studio (Wageningen UR) for help with the high temperature shear cell. We acknowledge Luuk Beekmans for his contribution to the experimental work. Part of the presented results are obtained using X-ray tomography equipment, which is owned by Shared Research Facilities and subsidized by Ministry of Economic Affairs and the province of Gelderland, The Netherlands. We thank ISPT for financial support. 


\section{Chapter 6}

Viscoelastic properties of soy protein isolate - pectin blends richer than those of a simple composite material

This chapter has been published as B.L. Dekkers, R.M. Boom \& A.J. van der Goot (2018). Viscoelastic properties of soy protein isolate - pectin blends: Richer than those of a simple composite material. Food Research International, 107, 281-288. http://doi.org/10.1016/j.foodres.2018.02.037 


\section{Abstract}

Concentrated soy protein isolate (SPI) - pectin blends acquire fibrous textures by shear-induced structuring while heating. The objective of this study was to determine the viscoelastic properties of concentrated SPI-pectin blends under similar conditions as during shear-induced structuring, and after cooling. A closed cavity rheometer was used to measure these properties under these conditions. At $140{ }^{\circ} \mathrm{C}$, SPI and pectin had both a lower $G^{*}$ than the blend of the two and also showed a different behaviour in time. Hence, the viscoelastic properties of the blend are richer than those of a simple composite material with stable physical phase properties. In addition, the $G_{\text {pectin }}^{\prime}$ was much lower compared with the $G_{S P I}^{\prime}$ and $G_{S P I-p e c t i n}^{\prime}$ upon cooling, confirming that pectin formed a weak dispersed phase. The results can be explained by considering that the viscoelastic properties of the blend are influenced by thermal degradation of the pectin phase. This degradation leads to: i) release of galacturonic acid, ii) lowering of the $\mathrm{pH}$, and iii) water redistribution from the SPI towards the pectin phase. The relative importance of those effects are evaluated. 


\subsection{Introduction}

Meat analogues based on plant proteins have the potential to contribute to a more sustainable diet through reducing the actual meat consumption. Meat analogues having a pronounced fibrous structure are known to be most appealing as alternative to meat for consumers (Hoek et al., 2011). Nowadays, high moisture extrusion is mostly used to make these types of meat analogues (Dubey \& Bhattacharya, 2015; Osen \& Schweiggert-Weisz, 2016). A decade ago, a structuring technology based on simple shear flow deformation was introduced for the development of fibrous proteinaceous structures with a meat-like appearance (Manski et al., 2007b). Plant-based biopolymers can be structured into fibres by using a concentrated two-phase system that consists either of two types of protein; soy protein isolate (SPI) and wheat gluten, or of a blend with protein and polysaccharides, which may be naturally present, as in soy protein concentrate (SPC) or assembled in a model system such as a SPI - pectin blend (Cheftel, Kitagawa, \& Queguiner, 1992; Dekkers, Hamoen, Boom, \& van der Goot, 2018; Dekkers, Nikiforidis, \& Goot, 2016; Grabowska et al., 2016; Grabowska, Tekidou, Boom, \& van der Goot, 2014; Liu \& Hsieh, 2007). Those immiscible biopolymer blends have a multiphase morphology in which the minor phase forms dispersed droplets. Both the dispersed phase droplets and the continuous phase absorb part of the water, and can therefore be described as a water-in-water emulsion. Similarly to regular emulsions, these droplets can coalesce, deform and break up upon shear-induced structuring. Deformation and break-up of droplets depend on the ratio between the external, viscous and internal or interfacial forces (Grace, 1982; Taylor, 1932). For two droplets to coalesce, they should approach each other and then expel the film in between (Elmendorp \& Van der Vegt, 1986; Lyu, Bates, \& Macosko, 2000). These forces and phenomena are dependent on the viscoelastic properties, the elasticity, and the interfacial tension of the blend (Elmendorp \& Van der Vegt, 1986; Verhulst, Cardinaels, Moldenaers, Afkhami, \& Renardy, 2009). Hence, determining these properties can contribute to a deeper understanding of fibrous structure formation processes.

Temperature has been shown to be a key process parameter when transforming protein blends into fibrous structures (Akdogan, 1999; Arêas, 1992; Cheftel et al., 1992; Grabowska et al., 2016). Heating results in a lower viscosity, which influences the flow conditions during high moisture extrusion (Osen \& Schweiggert-Weisz, 2016). Furthermore, temperature affects protein crosslinking reactions (Arêas, 1992). The temperature was also shown to be a key process parameter when structuring protein-polysaccharide blends, such as soy protein concentrate and SPI - pectin 
blends, with simple shear flow (Dekkers, Nikiforidis, et al., 2016; Grabowska et al., 2016). Distinct differences in both the micro- and macrostructure were observed when varying the heating temperature between $120^{\circ} \mathrm{C}$ and $140^{\circ} \mathrm{C}$ during structure formation of SPI - pectin blends (Dekkers et al., 2016). In this study, these observations on micro- and macrostructure will be connected to the viscoelastic behaviour of the separate components, being SPI and pectin, and the SPI-pectin blend at various temperatures.

In the SPI-pectin blend, the properties of the blend change over time due to chemical and physical instability of both components at elevated temperatures. Pectin is thermally unstable due to non-enzymatic degradation (Chen et al., 2015; Diaz et al., 2007; Shpigelman, Kyomugasho, Christiaens, Van Loey, \& Hendrickx, 2014; Thakur et al., 1997). At neutral pH, $\beta$ elimination leads to lowering of the molecular weight. Furthermore, both $\beta$-elimination and demethoxylation result in the release of acid groups resulting in lowering of the $\mathrm{pH}$ (Axelos \& Branger, 1993; Renard \& Thibault, 1996; Thakur et al., 1997). Heating of soy protein can induce rearrangement, aggregation, and degradation reactions (Huang, Chang, \& Jane, 1999; Kinsella, 1979). These reactions result in differences in the viscoelastic behaviour of the two-phases as separate components, and impact the deformability of the two phases during structuring. Secondly, the chemical and physical instabilities can affect the interactions between the twophases. For example, lowering of the $\mathrm{pH}$ of the blend due to $\beta$-elimination and demethoxylation affects aggregation of the protein phase. Degradation of the biopolymers also affects the water binding in the network and hence the water distribution between the two biopolymer phases. This is important since the concentration of the polymers in each phase will determine the actual viscosity in the respective, while also the relative volumes of the phases are important in the structure formation (Dekkers, Emin, Boom, \& van der Goot, 2018). Lastly, the chemical and physical changes affect the elasticity of the two-phases. The elasticities of both the droplet and the matrix influence the morphology of the dispersed phase. An elastic dispersed phase will result in droplets that are more resistant against droplet break-up under deformation, whereas elastic matrices result in more deformed droplets and a higher break-up rate (Elmendorp \& Maalcke, 1985; Lerdwijitjarud, Sirivat, \& Larson, 2002).

The objective of this study was to determine the viscoelastic properties of concentrated SPIpectin blends during and after applying a high temperature and shear treatment. The viscoelastic time-temperature dependency of the blend will be related to phenomena observed in shearinduced structuring, which was shown to yield fibrous structures (Dekkers et al., 2018; Dekkers, 
Nikiforidis, et al., 2016). The rheological properties under process conditions relevant for structuring cannot be studied with traditional rheometers, because of the extreme concentrations and temperatures. We therefore use a device developed for quantifying rubber rheology to determine the viscoelastic properties of concentrated dispersions of SPI and pectin at elevated temperatures over time. This device encloses the material in a cavity; by applying a closure pressure, water evaporation is prevented (Emin \& Schuchmann, 2017). A shear treatment is simulated by using high strain and high frequency.

\subsection{Materials \& methods}

\subsubsection{Materials}

Soy protein isolate (SUPRO® 500E IP) was obtained from Solae (St Louis, MO). The isolate contained at least 83.4 wt.\% protein ( $\mathrm{N}$ x 5.71). Pectin extracted from citrus peel (HM-pectin P9135, LOT\#SLBQ6929V) was obtained from Sigma-Aldrich (Germany). It has a galacturonic acid content of $\geq 74.0 \%$ on a dry basis and contains $\geq 6.7 \%$ methoxy groups. The degree of methyl esterification is around 70\% (Yoo, Fishman, Hotchkiss, \& Hyeon, 2006). The pH was adjusted using $\mathrm{HCl}$ and $\mathrm{NaOH}$, both purchased from Sigma Aldrich (Germany).

\subsubsection{Sample preparation}

Samples were prepared by first mixing the dry soy protein isolate (SPI) and/or pectin powder. $\mathrm{NaCl}$ was dissolved in the distilled water phase, after which this solution was slowly added to the dry powders followed by mixing shortly. The created blend consisted of $41.8 \mathrm{wt} . \%$ SPI, 2.2 wt. $\%$ pectin, 1 wt. $\%$ salt and 55 wt. $\%$ water. This ratio between SPI and pectin was used, since we found this ratio to be optimal for shear-induced structuring (Dekkers, Nikiforidis, et al., 2016). The separate components consisted of $44 \mathrm{wt} . \%$ SPI or pectin, $1 \mathrm{wt.} \%$ salt and $55 \mathrm{wt} . \%$ water unless stated otherwise. To change the $\mathrm{pH}$ of the blend, a $\mathrm{NaOH}$ or $\mathrm{HCl}$ solution was used instead of water. After mixing, the material was hydrated for at least $30 \mathrm{~min}$.

\subsubsection{Viscoelastic properties}

The viscoelastic properties of SPI, pectin, and SPI/pectin blends were determined with a rubber process analyser (RPA elite, TA instruments, USA), further referred to as a closed cavity rheometer (CCR). The material, approximately 5 gram, was placed in between two plastic foils in the closed cavity (disk geometry), which was sealed with a closing pressure of 4.5 bar to prevent water evaporation. Three types of measurements were performed. The first set of 
experiments consisted of a time-sweep, a cooling step and a frequency sweep. The time-sweep was performed at high strain $(80 \%)$ and high frequency $(10 \mathrm{~Hz})$ while heating at various temperatures $\left(110-140{ }^{\circ} \mathrm{C}\right)$ for $15 \mathrm{~min}$. During the first $30 \mathrm{sec}$, the temperature of the die was lowered due to loading of the samples. Thereafter the set-temperature was reached again (data shown from this point onwards). After the time-sweep, the material was cooled in approximately $12 \mathrm{~min}$ from the set temperature to $40{ }^{\circ} \mathrm{C}$ by blowing dry air over the disk. Subsequently, the linear viscoelastic regime (LVR) was determined by performing a strain sweep $(0.1-100 \%, 1 \mathrm{~Hz})$, and a frequency-sweep was performed in the LVR $\left(10-0.1 \mathrm{~Hz}, 1 \%\right.$ strain), both at $40{ }^{\circ} \mathrm{C}$. The second set of experiments was used to study the relaxation of materials. Therefore, a time-sweep experiment $(80 \%, 10 \mathrm{~Hz})$ was paused after set time points $(2.5,5,7.5,10,12.5 \mathrm{~min})$, and restarted again after $8 \mathrm{sec}$, for a total of $15 \mathrm{~min}$. The difference in the viscoelastic properties before and after pausing, were determined and used as an indicator for the relaxation behaviour of the material. The third set of experiments consisted of time-sweep experiments that were performed for $0.5,1,3,5,10$, and $15 \mathrm{~min}$. The material was cooled down to room temperature in plastic bags to determine the $\mathrm{pH}$ of these samples. The gelled materials were grinded and subsequently mixed with distilled water (1:1) to measure the $\mathrm{pH}$.

\subsubsection{Differential scanning calorimetry}

Thermal transitions were determined with differential scanning calorimetry (DSC) (Diamond DSC, PerkinElmer, Shelton, USA). The DSC was calibrated with indium, and an empty stainless steel pan was used as reference. Soy protein isolate was dispersed in water (20 wt.\%). The samples were heated from $20^{\circ} \mathrm{C}$ to $150{ }^{\circ} \mathrm{C}$ at $10^{\circ} \mathrm{C} / \mathrm{min}$ and heating cycle was repeated two times. Nitrogen was used as carrier gas. Measurements were analysed with Start Pyris Software (PerkinElmer, Shelton, USA).

\subsection{Results and discussion}

The key question in the first part of this paper is: how do temperature and shear influence the viscoelastic properties of a soy protein isolate (SPI) - pectin blend? First, the viscoelastic properties of separate SPI and pectin were investigated, after which the blends were studied. In these measurements, a high strain and high deformation frequency were applied in a closed cavity rheometer (CCR) to approach process conditions, i.e. a shear treatment, that are relevant from an application point of view. Consequently, the measurement is done outside the linear viscoelastic regime (LVR), which means that the resulting periodic stress waveform becomes 
distorted and deviates from a sinusoidal wave; therefore we refer to apparent $G^{\prime}, G^{\prime \prime}$ and $G^{*}$ values. After characterizing the materials at high temperature, we determined the viscoelastic behaviour upon cooling to $40{ }^{\circ} \mathrm{C}$ with a strain and frequency sweep in the LVR. In the second part of this paper, we describe and analyse the differences as observed in the viscoelastic properties between SPI and SPI-pectin during the shear treatment at the various temperatures, and relate it to shear-induced structuring.

\subsubsection{Viscoelastic properties at elevated temperatures}

The viscoelastic properties of pectin, SPI and SPI-pectin blends were determined during timesweep experiments with high frequency $(10 \mathrm{~Hz})$ and high strain $(80 \%)$ at elevated temperatures (110-150 $\left.{ }^{\circ} \mathrm{C}\right)$. In Fig. 6.1, the apparent storage $(G)$ and loss $\left(G^{\prime}\right)$ modulus of the three materials are shown at 120,130 and $140{ }^{\circ} \mathrm{C}$. The $G^{\prime}$ and $G$ ” of SPI at $120^{\circ} \mathrm{C}$ remained approximately constant $\left(G_{0.5 \text { min }}^{\prime}=8.4 \mathrm{kPa} \rightarrow G_{15 \text { min }}^{\prime}=9.0 \mathrm{kPa}\right)$. At $130{ }^{\circ} \mathrm{C}$, a slight decrease of $G^{\prime}$ was measured over time $\left(G_{0.5 \text { min }}^{\prime}=5.1 \mathrm{kPa} \rightarrow G_{15 \text { min }}^{\prime}=3.2 \mathrm{kPa}\right.$ at $\left.15 \mathrm{~min}\right)$. At $140{ }^{\circ} \mathrm{C}, G^{\prime}$ 'decreased even faster

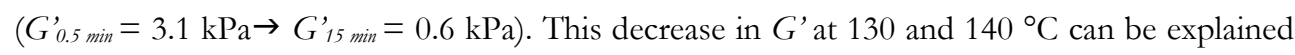
by degradation of SPI (Kinsella, 1979).

For all the three temperatures, the apparent $G$ ' and $G$ " of pectin decreased over time (Fig. 6.1B). At the start of each experiment, pectin behaved like an elastic solid $\left(G^{\prime} \approx 20-24 \mathrm{kPa}>G^{\prime \prime} \approx 12.5-\right.$ $15.5 \mathrm{kPa}$ ). It started to behave like an elastic liquid indicated by the fact that the $G$ ' decreased faster compared with the $G$ " resulting in a cross-over. The behaviour is indicative for degradation of pectin over time. The time to the cross-over point $\left(G^{\prime}=G^{\prime}\right)$ changed as a function of temperature: $483 \mathrm{~s}$ for $120^{\circ} \mathrm{C}, 126 \mathrm{~s}$ for $130{ }^{\circ} \mathrm{C}$, and $39 \mathrm{~s}$ for $140^{\circ} \mathrm{C}$. The values of $G^{\prime}$ and $G$ " at cross-over were approximately the same $(7.1 \mathrm{kPa})$. After the cross-over point, $G^{\prime}$ and $G$ ” decreased further and therefore the measuring limit of the CCR was approached at $130{ }^{\circ} \mathrm{C}$ and $140{ }^{\circ} \mathrm{C}$ after $15 \mathrm{~min}$. The $\mathrm{pH}$ of the pectin dispersion was 3.9 after this time. The $\mathrm{pH}$ of the pectin phase might be higher, when the pectin is dispersed in the protein phase. Therefore, we did some preliminary experiments, which showed that the $G^{\prime}$ and $G$ "values at $140{ }^{\circ} \mathrm{C}$ decreased faster when the $\mathrm{pH}$ was increased by the addition of $\mathrm{NaOH}$, which is expected to be a result of faster degradation of pectin at higher $\mathrm{pH}$ (results not shown).

Very different behaviour of the blend was found as compared with the two-phases separately (Fig. 6.1C). After the reduction of $G^{\prime}$ 'due to the initial heating, the $G^{\prime}$ of the SPI-pectin blend increased again over time. At $120^{\circ} \mathrm{C}$, the initial decrease due to heating resulted in a $G^{\prime}$ of 6.5 
$\mathrm{kPa}$ and over time the $G^{\prime}$ increased to a maximum of $8.1 \mathrm{kPa}$, which was reached after $12.2 \mathrm{~min}$. At $130^{\circ} \mathrm{C}$, the initial decrease due to heating resulted in a $G^{\prime}$ of $3.4 \mathrm{kPa}$ and over time the $G^{\prime}$ increased to a maximum value of $5.0 \mathrm{kPa}$, which was reached after $11.6 \mathrm{~min}$. At $140{ }^{\circ} \mathrm{C}$, the initial $G^{\prime}$ was $2.0 \mathrm{kPa}$ and over time the $G^{\prime}$ increased to the highest value of $3.1 \mathrm{kPa}$, which was reached after $8.8 \mathrm{~min}$. For the blend, initial heating seemed to take longer as compared to the separate components, since $G$ ' decreased during the first minute. The reason for this difference in heat transfer is unknown.
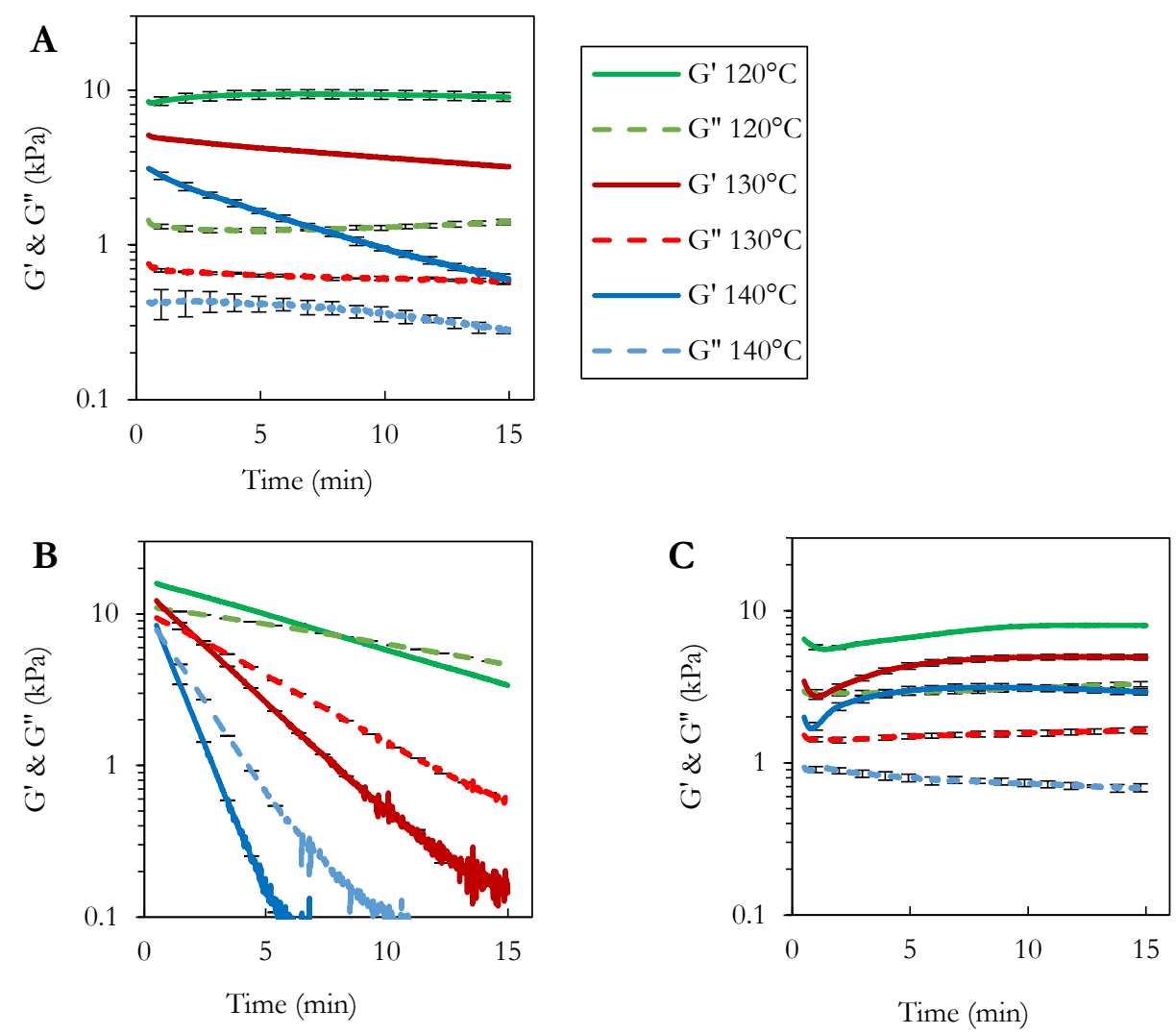

Fig. 6.1 Time-sweep measurements $(79.65 \%$ strain, $10 \mathrm{~Hz}$ frequency) at various temperatures; A) of soy protein isolate (SPI), B) pectin, C) SPI-pectin ( $n=3$, standard deviation plotted per minute)

Fig. 6.2 shows the apparent complex modulus $\left(G^{*}\right)$ of SPI, pectin and SPI-pectin in one graph for each temperature. At $120^{\circ} \mathrm{C}$, the $G^{*}$ of pectin was higher than the $G^{*}$ of SPI and of the SPIpectin blend during the first $10 \mathrm{~min}$. The degradation of pectin, however, resulted in a decrease of $G^{*}$ and therefore after $15 \mathrm{~min}$ the $G^{*}$ of pectin was slightly lower than that of SPI and SPIpectin. The $G^{*}$ of SPI and SPI-pectin were similar, which indicated that the addition of pectin 
had hardly any effect on the viscoelastic properties of the blend. At 130 and $140{ }^{\circ} \mathrm{C}$, the $G^{*}$ of pectin and SPI both decreased over time, whereas that of the blend increased.

An increased $G^{*}$ of the blend compared with its isolated constituents SPI and pectin, indicated that this blend could not be regarded as a simple composite material. According to polymer blending laws, developed by Takayanagi et al., the overall modulus of a simple composite is related to the moduli of the separate components (Morris, 2009; Takayanagi et al., 1963). In isostrain deformation, the continuous phase is the stronger component and therefore the strong continuous and weak dispersed phases are deformed to a similar degree. In isostress deformation, the continuous phase is the weaker phase, and hence the strength of the weak component limits the stress transmitted to the stronger dispersed phase. In both models, the strength of the blended material is never higher than the strength of one of the individual phases. Since the SPI-pectin blend is much stronger than both phases, it is obvious that other factors are needed to explain the viscoelastic properties of this blend.
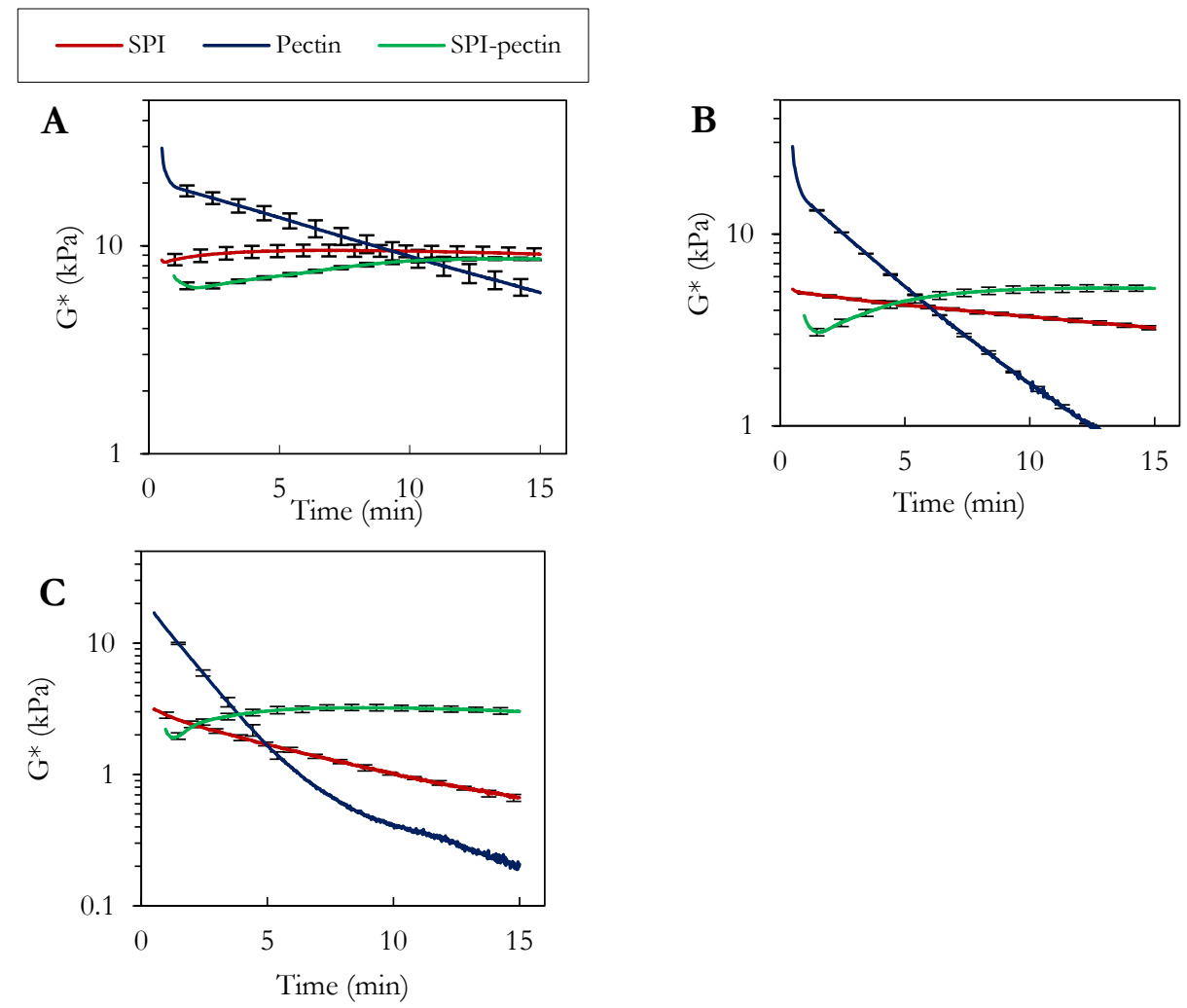

Fig. 6.2 Time-sweep measurements (79.65\% strain, $10 \mathrm{~Hz}$ frequency) for SPI, pectin and SPI-pectin at various temperatures A) $\left.120^{\circ} \mathrm{C}, \mathrm{B}\right) 130^{\circ} \mathrm{C}$, and C) $140^{\circ} \mathrm{C}(\mathrm{n}=3$, standard deviation plotted per minute) 
Fig. 6.3-A shows the apparent $G^{*}$ for SPI-pectin blends while heating at 120,130 and $140{ }^{\circ} \mathrm{C}$ and pausing and resuming the measurements after $7.5 \mathrm{~min}$. These measurements were used as an indication of the ability of the blend to recover after having been sheared. At 120 and $130{ }^{\circ} \mathrm{C}$, distinct peaks in $G^{*}$ were observed after resuming the measurement, whereas this peak almost disappeared at $140{ }^{\circ} \mathrm{C}$. Besides pausing and resuming the measurements after $7.5 \mathrm{~min}$, we also determined the difference in the complex modulus $\left(\Delta G^{*}\right)$ before and after pausing the measurements, i.e. the height of the small peak, at several other time points (Fig. 6.3B). During the first $7.5 \mathrm{~min}, \Delta G^{*}$ increased similarly for 120 and $130{ }^{\circ} \mathrm{C}$. At $120^{\circ} \mathrm{C}$, the $\Delta G^{*}$ increased slightly when pausing/resuming after $7.5,10$ or $12.5 \mathrm{~min}$, whereas at $130{ }^{\circ} \mathrm{C} \Delta G^{*}$ reached a plateau. The $\Delta G^{*}$ at $140{ }^{\circ} \mathrm{C}$ was much smaller and remained constant over time.

Similar experiments performed for the separate SPI and pectin did not yield any peaks in $G^{*}$ at 120 or $130{ }^{\circ} \mathrm{C}$ when pausing and resuming the measurements. At $140{ }^{\circ} \mathrm{C}$, the $G^{*}$ decreased slightly when pausing and resuming the measurements, which we expect to be a result of continuation of thermal degradation. These results indicate once more that the blend shows distinctly different viscoelastic behaviour than expected based on the properties of the two separate components.
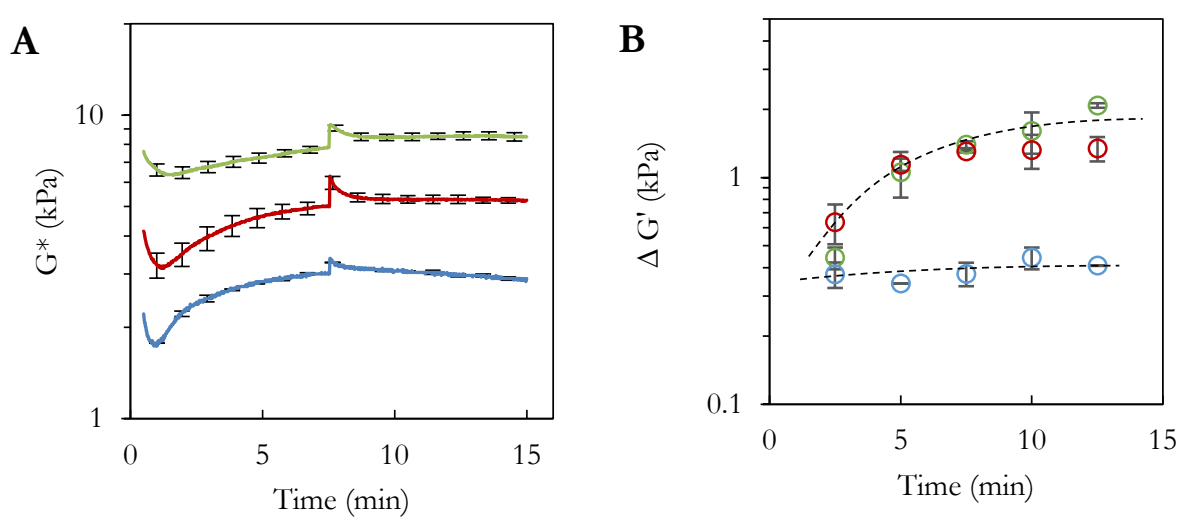

Fig. 6.3 Difference in $G^{*}$ after pausing and resuming a time-sweep experiment (79.65\% strain, $10 \mathrm{~Hz}$ frequency) in SPIpectin blends at $120^{\circ} \mathrm{C}(-,, 0), 130^{\circ} \mathrm{C}(-, \mathrm{O}), 140^{\circ} \mathrm{C}(-, \mathrm{O})$ A) example of time-sweep measurements stopped for 8 seconds after $7.5 \mathrm{~min}$, B) overview of peak height after pausing the measurement at various time points ( $n=2$, standard deviation plotted every minute) Lines are to guide the eye

In shear-induced structuring, the domain size of the dispersed droplets depend on the temperature; processing at $120{ }^{\circ} \mathrm{C}$ gives smaller dispersed pectin droplets compared with processing at $140^{\circ} \mathrm{C}$ (Dekkers, Nikiforidis, et al., 2016). This may be related to the ability of the elastic properties to recover after deformation, which is indicative of the presence of a physical 
network. Elasticity of the matrix can lead to instability of the dispersed droplets, resulting in more break-up during processing (Elmendorp \& Maalcke, 1985; Lerdwijitjarud et al., 2002). We can thus conclude that when high strain and high frequency are applied, the blend is able to recover at 120 and $130^{\circ} \mathrm{C}$, whereas the ability of the blend to recover becomes lower at $140{ }^{\circ} \mathrm{C}$ in the time span. This may explain the presence of the larger droplets observed at $140{ }^{\circ} \mathrm{C}$ as compared with 120 and $130{ }^{\circ} \mathrm{C}$ (Dekkers, Nikiforidis, et al., 2016).

\subsubsection{Viscoelastic properties after cooling}

After the time-sweep experiments, the CCR was cooled to $40^{\circ} \mathrm{C}$. At this temperature, a strainsweep was performed to determine the LVR (supplemental information) and a frequency-sweep was performed (Fig. 6.4). This measurement allows the comparison of the influence of the temperature during the shear treatment upon cooling, and allows characterization of these materials in a conventional way. We determined the dry matter content after these measurements and we observed that minimal water losses had occurred. In Fig. 6.4A-C, an overview of the low-temperature frequency-sweeps is shown for SPI, pectin, and SPI-pectin. For both SPI and SPI-pectin, the $G^{\prime}$ and $G$ " values increased when the time-sweep experiment was performed at 120 and $130^{\circ} \mathrm{C}$. The values were lower for $140^{\circ} \mathrm{C}$. The absolute difference between $G$ ' and $G$ " decreased with increasing temperature. We expect that the proteins aggregate more strongly up to a temperature of $130{ }^{\circ} \mathrm{C}$, resulting in a stronger network. At $140{ }^{\circ} \mathrm{C}$, a lower $G^{\prime}$ value was found, possibly related to protein degradation. Thermal transitions of SPI were also determined with differential scanning calorimetry, which showed an exothermic peak at $131{ }^{\circ} \mathrm{C}$, related to thermal degradation (Huang et al., 1999; Kinsella, 1979). For pectin, the G' and G” decreased with increasing temperature, which is expected due to the degradation ( $\beta$-elimination) at elevated temperatures (Chen et al., 2015; Diaz et al., 2007; Shpigelman et al., 2014; Thakur et al., 1997).

In Fig. 6.4-D, an overview of the $G^{\prime}$ values at $1 \mathrm{~Hz}$ is shown after performing the time-sweep experiment at a broader range of temperatures. It shows that the difference in $G$ 'between pectin, and SPI and SPI-pectin increased with increasing temperatures. These results show that pectin forms a weak phase upon cooling, as hypothesized in our previous study (Dekkers, Nikiforidis, et al., 2016). Fig. 6.4E shows the $G^{\prime}$ of SPI and SPI-pectin on a different scale for a better comparison. At 110 and $120^{\circ} \mathrm{C}$, the $G^{\prime}$ of SPI was slightly higher than the G' of SPI-pectin, which indicates that the pectin alters the properties of the SPI-phase in the blend at these temperatures. At $130{ }^{\circ} \mathrm{C}$, the $G^{\prime}$-values of SPI and SPI-pectin were similar, while above $130{ }^{\circ} \mathrm{C}$ the G' of SPI was lower than the G'of SPI-pectin. 
A

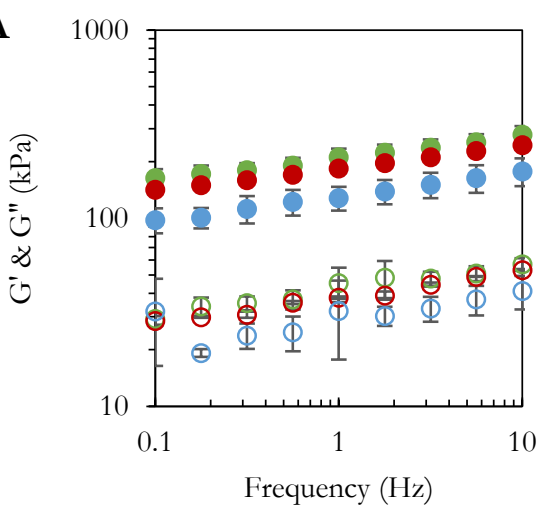

C

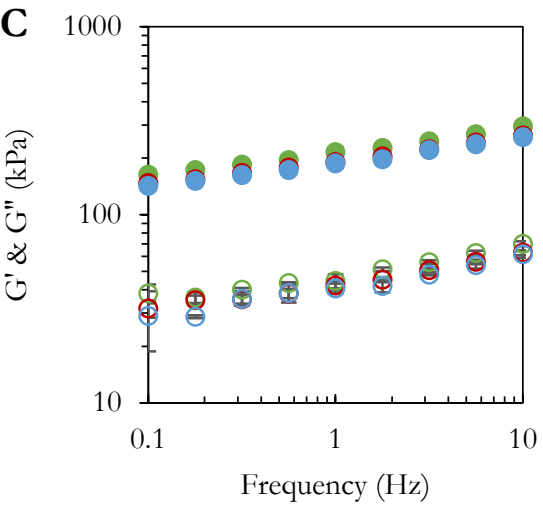

$\mathbf{E}$

E

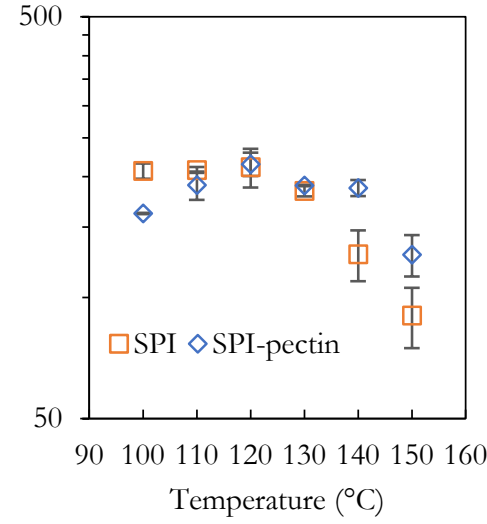

B

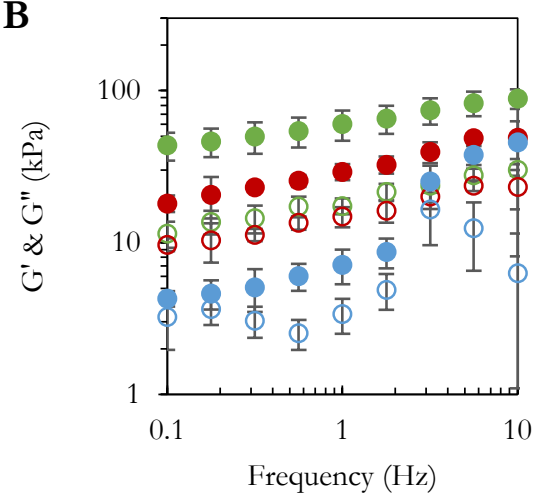

D

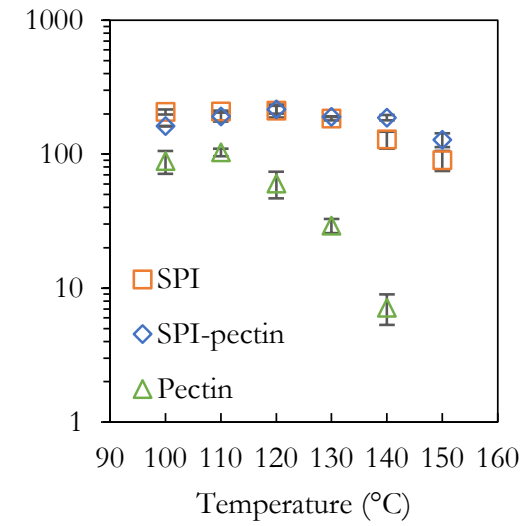

Fig. 6.4 Frequency-sweep (10-0.1 $\mathrm{H}_{\%}, 1 \%$ strain, $\left.40^{\circ} \mathrm{C}\right)$ results after performing a time-sweep with bigh strain and frequency at: $120(\bigcirc), 130(-), 140^{\circ} \mathrm{C}(\bigcirc)$ for A) Soy protein isolate (SPI), B) pectin and C) SPI-pectin blend, and D) and E) show an overview of $G^{\prime}$ of SPI, pectin and the blend at $1 \mathrm{~Hz}_{2}$ at various temperatures $(n=3)$

At $140^{\circ} \mathrm{C}$, the SPI-pectin blend behaved distinctly different compared with the single components; the $G$ ' of the blend was higher than those of the single components. Addition of pectin, being a dispersed phase with a $G$ ' lower than the continuous SPI phase, should 
theoretically result in lowering of the $G^{\prime}$ of the blend when it would be a simple composite material. We can conclude that this is not the case. We found that pectin degrades at $140{ }^{\circ} \mathrm{C}$, which results in lower moduli over time, and also in i) lowering of the $\mathrm{pH}$ of the blend, ii) release of hydrolysis products, such as galacturonic acid, and iii) water redistribution, i.e. more water is absorbed by the pectin phase due to degradation (depolymerization leads to smaller molecules which leads to more water binding overall) and therefore a more concentrated continuous phase is obtained. We hypothesize that these effects overcome the intrinsic decrease in viscosity that the pectin phase itself exhibits.

\subsubsection{The $\mathrm{pH}$ effect}

Pectin is unstable at elevated temperatures: depolymerization of the pectin will cause a decrease in $G^{\prime}$. Furthermore, $\beta$-elimination and demethoxylation result in the release of acid groups resulting in lowering of the $\mathrm{pH}$ (De Roeck et al., 2009; Fraeye et al., 2007), which influences the viscoelastic properties of the SPI-phase in the blend. We therefore determined the $\mathrm{pH}$ of the blend after performing a time-sweep experiment at various temperatures, at several time points. In Fig. 6.5, the $\mathrm{pH}$ of the heated SPI $\left(140^{\circ} \mathrm{C}\right)$ and SPI-pectin $\left(120,130\right.$ and $\left.140{ }^{\circ} \mathrm{C}\right)$ are shown as functions of the processing time. The SPI dispersion and SPI-pectin blend had already a different $\mathrm{pH}$ after mixing the two biopolymers $(t=0 \mathrm{~min}$ ), being 6.9 and 6.7 , respectively. The $\mathrm{pH}$ of SPI first increased slightly when performing the time-sweep experiment for $3 \mathrm{~min}$, and thereafter the $\mathrm{pH}$ decreased slightly $(6.9 \rightarrow 6.7)$ at $140{ }^{\circ} \mathrm{C}$. The $\mathrm{pH}$ of the SPI-pectin blends showed a marked decrease in $\mathrm{pH}$ over time. The $\mathrm{pH}$ decreased faster at higher temperatures; at $120{ }^{\circ} \mathrm{C}$ the $\mathrm{pH}$ decreased from $6.7 \rightarrow 6.4$, at $130^{\circ} \mathrm{C}$ the $\mathrm{pH}$ decreased from $6.7 \rightarrow 6.3$, and at $140{ }^{\circ} \mathrm{C}$ the $\mathrm{pH}$ decreased from $6.7 \rightarrow 6.1$ after $15 \mathrm{~min}$.

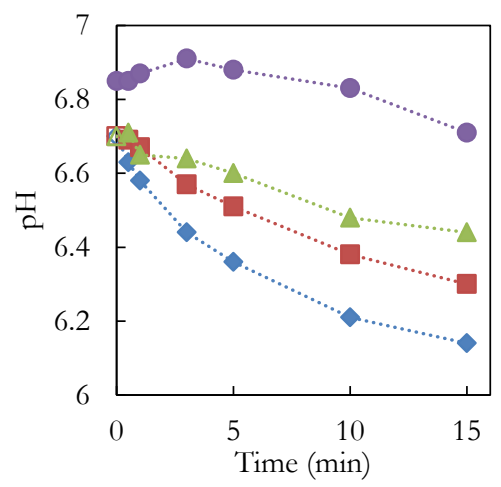

Fig. 6.5 pH of samples after processing in the closed cavity rheometer at various temperatures for SPI-pectin at: $120^{\circ} \mathrm{C}(\Delta)$, $130{ }^{\circ} \mathrm{C}(\square), 140{ }^{\circ} \mathrm{C}(\diamond)$, and SPI at $140^{\circ} \mathrm{C}(\mathbf{0})$ 
SPI was dispersed in varying concentrations of acid $(\mathrm{HCl})$ to determine whether the decrease in $\mathrm{pH}$ explains the increase in viscosity, which was observed in the SPI-pectin blend. Fig. 6.6 shows that the $G^{*}$ of SPI samples increased at a lower $\mathrm{pH}$. When we simulated the $\mathrm{pH}$ of the SPIpectin blend in the SPI dispersion, an increase in modulus was observed just as with the blend during the first few min. After $15 \mathrm{~min}$, SPI with a $\mathrm{pH}$ of 5.8, being slightly lower than that of the SPI-pectin blend $(\mathrm{pH}=6.1)$, had approximately the same $G^{*}(2.58 \mathrm{kPa})$. Hence, the decrease in $\mathrm{pH}$ cannot be the only explanation for the increase in viscoelastic properties of the blend. Nevertheless, it is clear that the gradual reduction in $\mathrm{pH}$ is a very important effect in explaining the rheological changes over time.
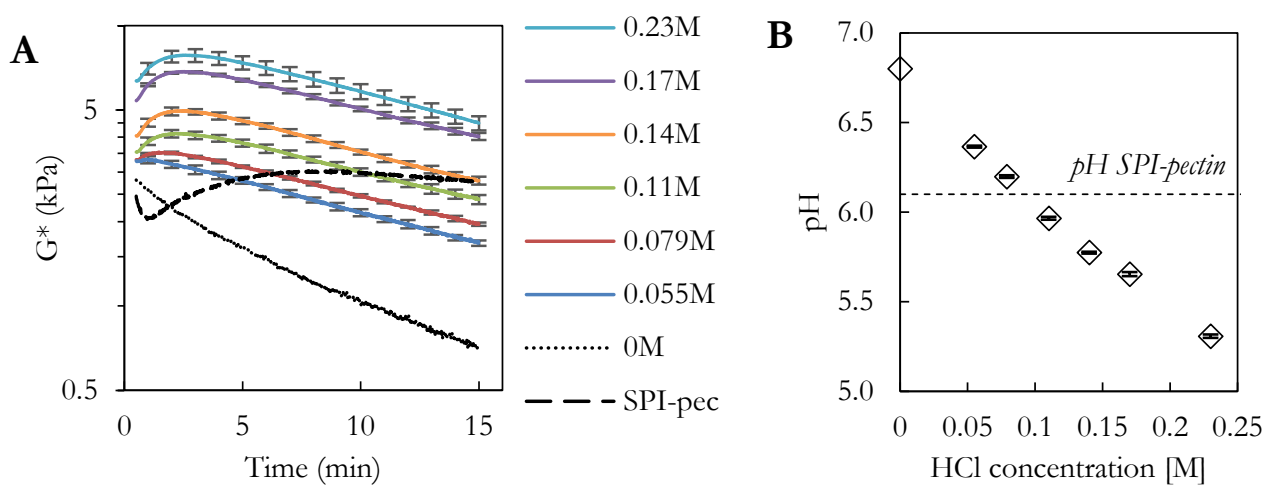

Fig. 6.6 Soy protein isolate (SPI) (41.8 wt.\%) with various concentrations of $\mathrm{HCl}$ and the SPI-pectin blend A) time-sweep measurements $\left(79.65 \%\right.$ strain, $10 \mathrm{~Hz}$ frequency) at $\left.140{ }^{\circ} \mathrm{C}, \mathrm{B}\right) \mathrm{pH}(\mathrm{n}=3)$

\subsubsection{Release of galacturonic acid}

Over time, $\beta$-elimination of pectin results in the release of polymeric and monomeric galacturonic acid subunits. To determine whether the release of galacturonic acid is a factor in the increase in $G^{*}$ of SPI-pectin blend at $140{ }^{\circ} \mathrm{C}$, we added various concentrations of galacturonic acid to SPI. The viscoelastic properties of the SPI-galacturonic acid blends are shown in Fig. 6.7. Indeed, we see that the addition of galacturonic acid increased the $G^{*}$. In Fig. 6.7A, varying concentrations of galacturonic acid were added to $41.8 \mathrm{wt} . \%$ SPI. A small addition of galacturonic acid (0.2 wt.\%) shifted the SPI curve to a higher $G^{*}$ as compared to 41.8 and 44 wt. $\%$ SPI. At higher concentrations of galacturonic acid (> $0.4 \mathrm{wt} . \%)$, an initial increase in $G^{*}$ was even observed during the first $5 \mathrm{~min}$. An increase in $G^{*}$ was also seen for the SPI-pectin blend, however the shape of the curve, i.e. the time-dependency of $G^{*}$, was different. This can be explained by the cumulative effects of the $\mathrm{pH}$ and of the release of galacturonic acid in the blends. The final $G^{*}$ after 15 min was comparable for SPI with 0.8 wt. $\%$ galacturonic acid. In 
these experiments, the dry matter content was not kept constant, and therefore we also did experiments with a total dry matter of 44 wt. $\%$ and changed the ratio between SPI and galacturonic acid. The $G^{*}$ after initial heating was higher compared with SPI only. The shape of the curve with a ratio between SPI and galacturonic acid of 43.2/0.8 was comparable to the SPIpectin curve, though shifted upwards.

A concentration of $0.8 \mathrm{wt} . \%$ galacturonic acid approximately corresponds with $45 \%$ of the pectin being fully degraded to monomeric subunits of galacturonic acid. The $\beta$-elimination reaction can only occur with the presence of a methyl group on the C6 of galacturonic acid (Albersheim \& Neukom, 1960). The degree of methylation of the citrus peel pectin used in these experiments is approximately $60 \%$, implying that $45 \%$ degradation is possible. However, our result might also be a combined effect of $\mathrm{pH}$ and the release of galacturonic acid, since the $\mathrm{pH}$ of the blends with addition of galacturonic acid was lower as compared to the final $\mathrm{pH}$ of the SPI-pectin blend.
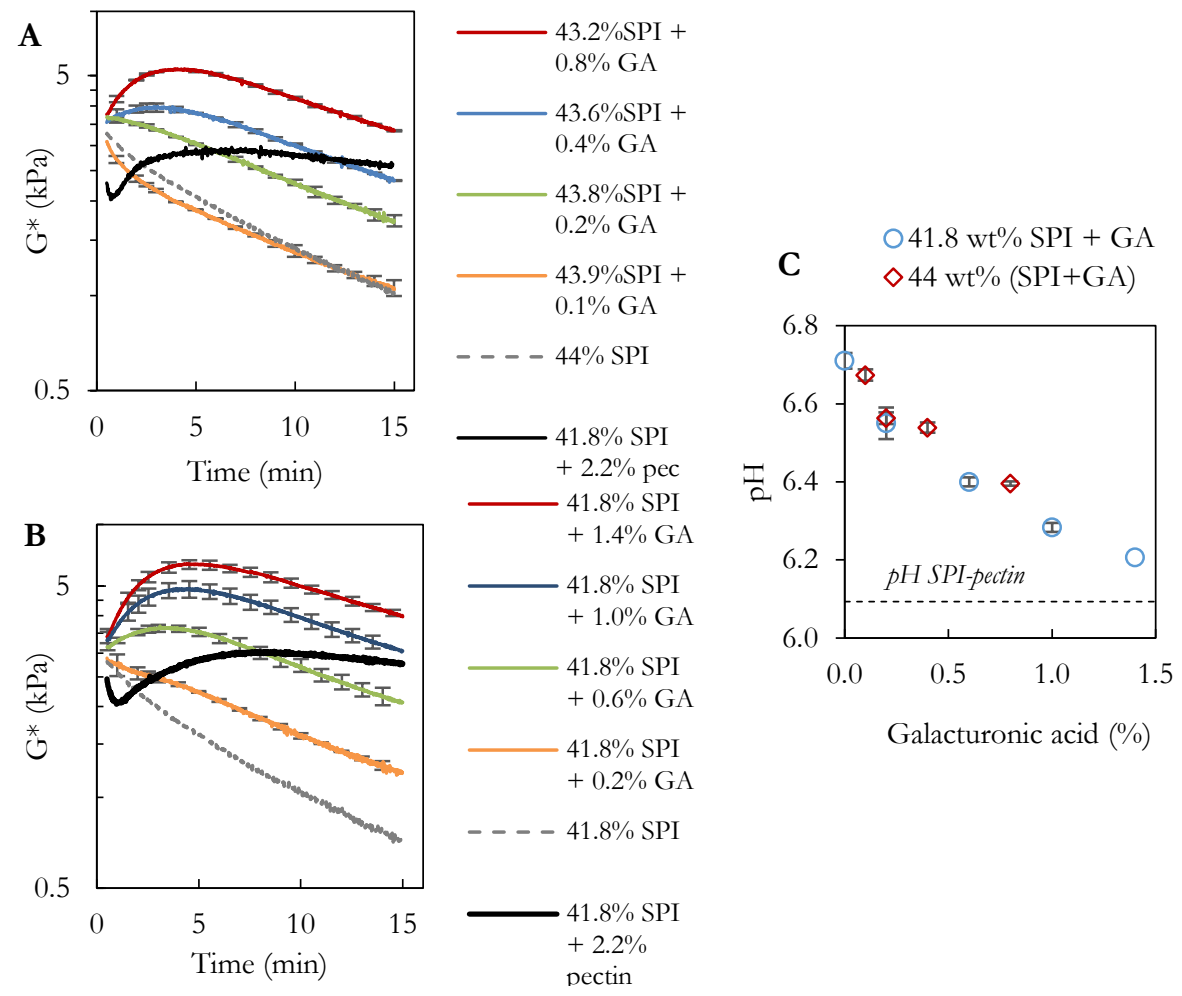

Fig. 6.7 The effect of galacturonic acid (GA) in a blend with soy protein isolate (SPI) A) time-sweep experiment $(79.65 \%$ strain, $10 \mathrm{~Hz}$ frequency) for SPI with the addition of various concentrations of galacturonic acid, B) $44 \mathrm{wt} \%$ (SPI/galacturonic acid) at various ratios, and C) $p H$ of SPI blend with galacturonic acid ( $n=3$ ) 


\subsubsection{Water redistribution}

In the previous study, we showed that the domain size of the dispersed droplets after shearing of SPI-pectin blends at various temperatures was larger in case of $140{ }^{\circ} \mathrm{C}$ than for 120 and 130 ${ }^{\circ} \mathrm{C}$ (Dekkers, Nikiforidis, et al., 2016). The increased size of the pectin domains may be the result of shear-induced coalescence, but also additional water absorption by the pectin phase might play a role (Dekkers et al., 2016; Dekkers, Emin, et al., 2018). This effect will increase the protein concentration in the SPI phase according to the mass balance depicted in Fig. 6.8A. This mass balance shows how the moisture content in the two phases change when water redistributes from the SPI phase into the pectin phase; when more water is absorbed by the pectin phase as compared to the SPI phase, then the concentration (expressed as dry matter fraction) of SPI becomes higher in the SPI phase and the dry matter fraction becomes lower in the pectin phase. In this mass balance, it is assumed that the SPI and pectin are completely phase separated. The viscoelastic properties of various concentrations of SPI were measured (Fig. 6.8B). To test the hypothesis whether the effect of water distribution can explain the marked increase in viscosity of SPI-pectin over time, we assume here that the contribution of the pectin phase when even more diluted would be very low and has therefore a negligible influence on the overall $G$ ' of the blend. The $G^{*}$ of the SPI-pectin blend corresponds to a $G^{*}$ of a SPI-concentration of approximately $50 \mathrm{wt} . \%$. Based on the mass balance, this would mean that the concentration of pectin in its phase is $17 \mathrm{wt} . \%$ and a volume fraction of 0.83 for SPI and 0.17 for pectin. It implies a large migration of water from the SPI phase into the pectin phase in case water redistribution is fully responsible in explaining the changes.
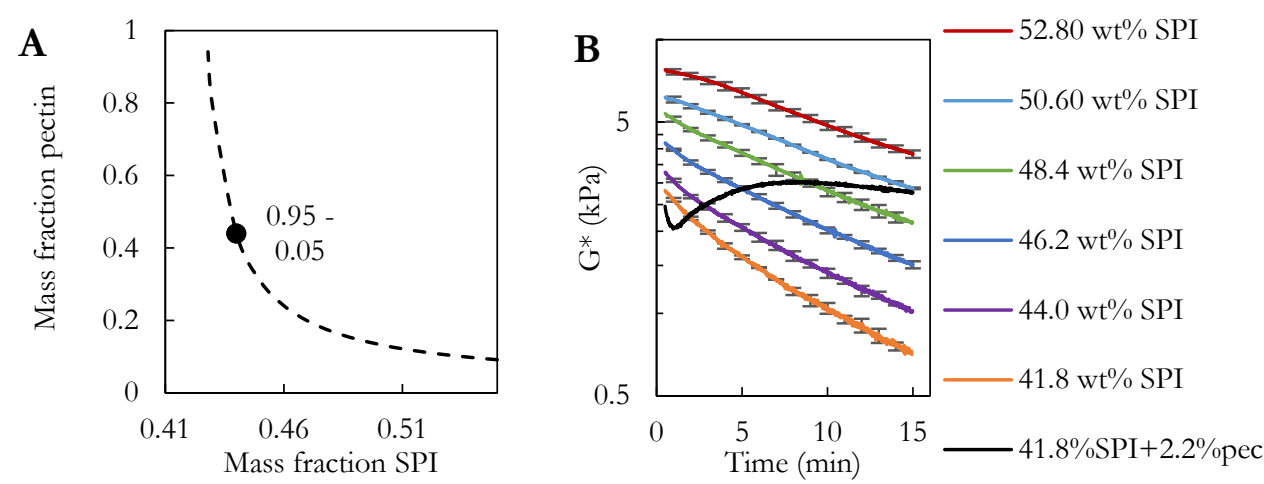

Fig. 6.8 The effect of water distribution in soy protein isolate (SPI) - pectin blends A) mass fraction of SPI and pectin in respective phase for various water distribution, even water distribution is indicated with $\mathbf{O}$, and B) time-sweep measurements (79.65\% strain, $10 \mathrm{~Hz}$ frequency) at $140^{\circ} \mathrm{C}$ for various concentrations of SPI $(n=3)$ 
All the three hypothesis that were tested to explain the differences between the separate components SPI and pectin, and the SPI-pectin led to an increase in the viscoelastic properties; decreasing the $\mathrm{pH}$ of the blend, addition of galacturonic acid, and water redistribution from the SPI to the pectin phase. When assuming that not all the pectin was degraded, then the pectin phase should theoretically result in lowering of the viscoelastic properties. All the three effects are contributing to the increased viscoelastic properties of the SPI-pectin blend. However, how these contribution are balanced is yet to be determined.

\subsection{Conclusion}

The viscoelastic properties of soy protein isolate (SPI), pectin and SPI-pectin blends were measured at relevant conditions for shear-induced structuring: concentrated (41.8 - $52 \mathrm{wt} . \%)$ and at elevated temperatures $\left(100-150{ }^{\circ} \mathrm{C}\right)$. At these elevated temperatures, the viscoelastic properties of SPI-pectin blends were distinctly different from the viscoelastic properties of SPI alone. The viscoelastic properties of the blend are richer than those of a simple composite material, with stable physical phase properties. Upon cooling, there were slight differences between SPI and SPI-pectin, whereas at $140{ }^{\circ} \mathrm{C} G_{\text {pectin }}^{\prime}<<G_{S P I}^{\prime}$ and $G_{S P I-p e c t i n}^{\prime}$, which indicates that pectin forms a weak dispersed phase. On the basis of our measurements, we propose that the differences in G' of SPI and SPI-pectin are the result of the combined effects of three mechanisms: i) a decrease in $\mathrm{pH}$ due to $\beta$-elimination and/or demethoxylation, ii) the release of galacturonic acid, and iii) water redistribution between the two-phases due to degradation of the soy proteins and pectin.

\section{Acknowledgements}

The authors would like to thank Dr M. Azad Emin from Karlsruhe Institute of Technology (KIT) for fruitful discussions. We acknowledge Martin Schreiber for his contribution to the experimental work. This research is part of the project Plant Meat Matters, which is co-financed by Top Consortium for Knowledge and Innovation Agri \& Food by the Dutch Ministry of Economic Affairs; the project is registered under contract number TKI-AF-16011. 


\section{Appendix}
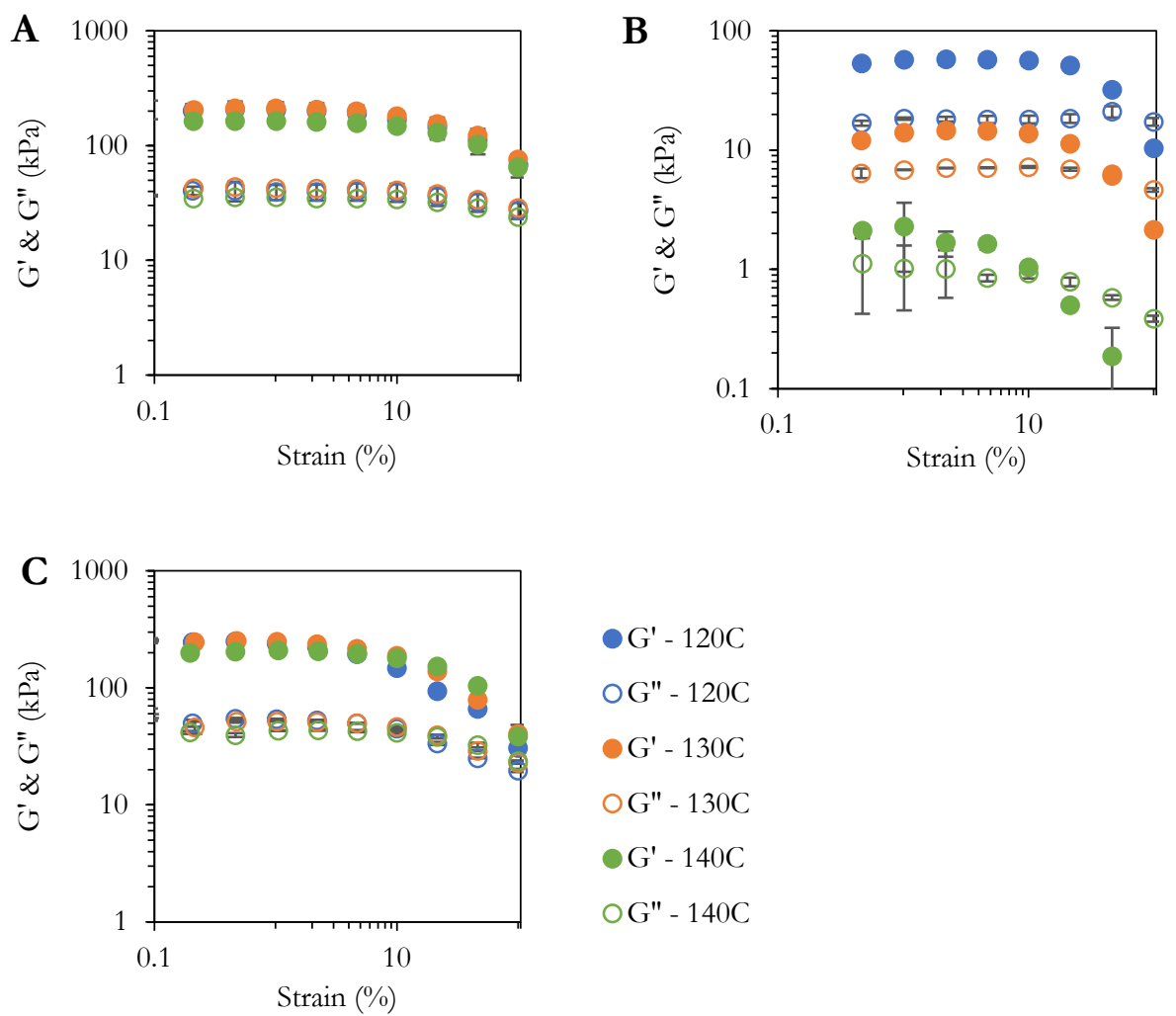

Fig. A1 Strain-sweep $\left(0.1-100 \%\right.$ strain, $\left.1 \mathrm{H}_{2}, 40^{\circ} \mathrm{C}\right)$ results after performing a time-sweep with high strain and frequency for A) soy protein isolate (SPI), B) pectin and C) SPI/pectin ( $n=2)$ 


\section{Chapter 7}

Aqueous fractionation processes of soy protein for fibrous structure formation

This chapter has been published as M. E. J. Geerts ${ }^{1}$, B.L. Dekkers ${ }^{1}$, A. van der Padt \& A.J. van der Goot (2017). Aqueous fractionation processes of soy protein for meat analogues. Innovative Food Science and Emerging Technologies, 45 (September 2017), 313-319. http://doi.org/10.1016/j.ifset.2017.12.002

${ }^{1}$ Authors contributed equally 


\section{Abstract}

Desired properties of ingredients differ for various applications. Here, we use a reverse engineering approach to obtain soy protein fractions targeted for the application of meat analogues. Aqueous fractionation was used to produce these soy protein fractions, which were structured with simple shear flow deformation while heating. The water holding capacity (WHC), nitrogen solubility index (NSI), enthalpy of transition, and viscoelastic properties were determined. We found that a soy protein fraction / full fat flour blend resulted in distinct fibrous structures but only when the soy protein fraction was toasted at $150^{\circ} \mathrm{C}$. At this optimum toasting temperature $\left(150{ }^{\circ} \mathrm{C}\right)$, the protein fractions had a high WHC, intermediate NSI and its viscoelastic property was characterized as $G^{*}$ between $1-10 \mathrm{kPa}$. These functional properties were shown to be key for fibrous structure formation, whereas, the influence of the state of the proteins was limited. 


\subsection{Introduction}

Ingredient production often aims at general applications, which requires defined chemical composition, and a stable product form such as powder. Traditionally, solubility is the targeted functional property for protein ingredients to allow applicability in drinks, emulsions and doughs (Zayas, 1997). Currently, meat analogues is a growing application area. For this application proteins should not solubilize in water, but bind water to allow the creation of a structure. Hence, considering a specific application, one could end up with other or additional requirements for functional properties. Therefore, it can be stated that modern fractionation methods should be designed while keeping a final application in mind. Here, we use fibrous protein structures, which could form the basis for meat analogues as an example to demonstrate a reverse engineering approach to develop ingredients. Soy flour is taken as starting material, because soy-based ingredients are used in many meat analog products currently on the market (Boland et al., 2013; Malav, Talukder, Gokulakrishnan, \& Chand, 2015).

Nowadays, meat analogues that are mimicking the fibrous structure of meat are produced with two extrusion processes; low moisture or high moisture. The extruder is used to form the fibrous structure, which is further process into a full meat analogy by freezing and frying (Giezen, Jansen, \& Willemsen, 2014). Another innovative technique based on simple shear flow deformation while heating was introduced a decade ago to produce fibrous structures from caseinate (Manski et al., 2007b). This concept was later applied to structure soy protein concentrate with a relatively high moisture content (55 wt.\%) (Grabowska et al., 2016). For fibrous structure formation with high moisture content, it is known that water absorption and gelling are important properties (Asgar et al., 2010; Singh, Kumar, Sabapathy, \& Bawa, 2008). Besides protein, other components can contribute to this functionality as well, and might even be required, given the hypothesis that a two phase system is a needed for structuring plant proteins into fibres (Cheftel et al., 1992; Grabowska et al., 2016).

Meat analogues that are currently on the market are consists of commercially available protein concentrates and isolates. These concentrates and isolates are mostly prepared with a conventional fractionation process of soy beans, which primary aim was the extraction of oil (Islas-Rubio \& Higuera-Ciapara, 2002). Oil is extracted from the soybean meal with organic solvents, but the consumer acceptance of those solvents is decreasing amongst others for environmental reasons (Dunn, Wells, \& Williams, 2010). The defatted soy flour is then further processed into protein concentrates or isolates for food applications (Day, 2013; Mulder, Peet- 
Schwering, Hua, \& Ree, 2016). Partial oil extraction can also be achieved using aqueous fractionation, which might be preferred by current consumers of meat analogues, who are caring about the environment (Hartmann \& Siegrist, 2017).

Aqueous fractionation is a method in which milled soy flour is mixed with excess water. The application of a centrifugation step yields three phases; i) a cream layer, which is rich in oil, ii) a liquid phase, which is rich in protein, and iii) a pellet, rich in insoluble fibres. The aqueous phase can be used as starting material for protein fractionation by acid precipitation (Campbell et al., 2011; De Almeida, De Moura Bell, \& Johnson, 2014; Russin, Boye, Arcand, \& Rajamohamed, 2011). Soy beans or soy flour are often toasted to decrease the enzyme activity and antinutritional factors (Kakade, Rackis, McGhee, \& Puski, 1974), but this toasting step also lowers the solubility (Onimawo \& Akpojovwo, 2006; Wu \& Inglett, 1974). Clearly, reduced solubility is undesired in the first steps of the fractionation process, because the method is based on solubility (Berk, 1992).

In this study, an aqueous soy protein fractionation process was designed specifically targeted to make fractions that can be structured into fibres for the application of meat analogues. The aim of this study was to reveal the essential functional properties of ingredients required for fibrous structure formation. To reveal these functional properties, a reversed engineering approach was used. Various soy protein fractions were mixed with water, and structured with a high temperature shear cell. We determined the functional properties of fractions in terms of: water holding capacity, nitrogen solubility, and enthalpy of transition (state of the proteins). The viscoelastic properties of the soy protein fractions in water were determined at similar conditions as used during the structuring process. The fractions were compared with commercially available soy protein concentrates (SPC) and isolates (SPI).

\subsection{Materials and Methods}

\subsubsection{Material}

Soy protein isolate (SPI, SUPRO® 500E IP) and soy protein concentrate (SPC, Alpha 6 IP) were both obtained from Solae (Europe S.A.). The manufacturer's specifications indicated that the SPI contained at least $83.4 \mathrm{wt} . \%$ protein and SPC contained at least $63.1 \mathrm{wt} . \%$ of protein $(\mathrm{Nx} 5.7)$. The $\mathrm{pH}$ was adjusted using $\mathrm{HCl}$ and $\mathrm{NaOH}$, both purchased from Sigma Aldrich (Germany). 


\subsubsection{Methods}

\subsubsection{Preparation of soy flour}

Soybeans were pre-milled into grits using a pin mill (LV 15M, Condux-Werk, Wolfgang bei Hanau, Germany). Subsequently, the soy bean grits were milled using a ZPS50 impact mill (Hosokawa-Alpine, Augsburg, Germany). The impact mill was set at a feed rate of 2-5 rpm, a speed of $8000 \mathrm{rpm}$, an airflow of $80 \mathrm{~m}^{3} / \mathrm{h}$ and a classifier wheel speed of $2500 \mathrm{rpm}$. A thermocouple inside the mill was used to monitor the temperature, which remained between 16 and $34{ }^{\circ} \mathrm{C}$.

\subsubsection{Aqueous fractionation}

A soy protein fraction was prepared by suspending soy flour in water $(20 \mathrm{wt} . \%)$ and adjusting the $\mathrm{pH}$ between 8 and 9 with $1 \mathrm{~mol} / \mathrm{L} \mathrm{NaOH}$ solution. The suspension was stirred for 1 hour and subsequently centrifuged $\left(10,000 \mathrm{~g} ; 30 \mathrm{~min} ; 20^{\circ} \mathrm{C}\right)$. The aqueous samples were poured through a cheese-cloth to separate the cream layer from the supernatant. The supernatant was collected and the $\mathrm{pH}$ was adjusted to 4.8 with $1 \mathrm{~mol} / \mathrm{L} \mathrm{HCl}$. The solution was stirred for at least 1 hour and subsequently centrifuged $\left(10,000 \mathrm{~g}, 30 \mathrm{~min} ; 20^{\circ} \mathrm{C}\right)$. The pellet was collected and neutralized ( $\mathrm{pH} 6.5$ - 7) with $1 \mathrm{~mol} / \mathrm{L} \mathrm{NaOH}$ and subsequently freeze dried (Christ, Germany). In this study, the supernatant was discarded.

\subsubsection{Toasting}

Soy flour and the soy protein fractions were toasted by spreading the powders over an oven tray ensuring an equal distribution of the flour of around $5-10 \mathrm{~mm}$ thick. The oven tray was placed in an Heratherm oven (Thermoscientific, USA) at various temperatures $\left(50-200^{\circ} \mathrm{C}\right)$ for $15 \mathrm{~min}$. Subsequently, the samples were cooled till room temperature and stored in a closed container at $4{ }^{\circ} \mathrm{C}$ up until further use.

\subsubsection{Shear-induced structuring with a high temperature shear cell}

A high temperature shear cell was used to structure the soy protein fractions. The soy protein fractions alone and soy protein fractions mixed with soy flour powder in a ratio 70/30 were used for structuring. For the preparation of the sheared samples, salt (1 wt. $\% \mathrm{NaCl}$ in the total blend) was dissolved in demineralized water. The powders were then added to the salt solution and thoroughly mixed to obtain a mixture with $44 \%$ dry matter. A hydration time of 30 min was used. The hydrated materials were placed into a preheated high temperature shear cell (HTSC) at $140{ }^{\circ} \mathrm{C}$, and sheared for $15 \mathrm{~min}$ at $30 \mathrm{rpm}$. The HTSC was developed in house (Grabowska 
et al., 2016; Peighambardoust et al., 2004). It consists of a rotating bottom cone and a stationary cone. An oil bath (JULABO LH46, USA) filled with Thermal H10 oil (JULABO, Germany) was used to heat and cool the cones, while a thermocouple measured the temperature inside the cone. A Haake drive (Haake Polylab QC, Germany) was used to control rotation speed (at 30 rpm). The HTSC was cooled down to room temperature within 5 min, before the samples were taken out. Samples were kept at room temperature for one hour prior to tensile strength analysis.

\subsubsection{Tensile strength analysis}

The degree of anisotropy was determined by cutting tensile bars parallel and perpendicular to the shear flow. From each sample, three tensile bars were taken in parallel and perpendicular. A texture analyzer (INSTRON 5564, USA) was used to deform the samples with a constant deformation rate of $1 \mathrm{~mm} / \mathrm{s}$. Samples were placed between two sand-coated clamps at a distance of $15.5 \mathrm{~mm}$. From the stress-strain curve we determined the Young's modulus, tensile stress and tensile strain at rupture. The ratio between the measures (Young's modulus, tensile stress, tensile strain) in parallel and perpendicular direction is referred to as the anisotropic index (AI), which is used as an indication of fibrousness of the material.

\subsubsection{Composition analysis}

The protein content was determined using Dumas analysis (Nitrogen analyser, FlashEA 1112 series, Thermo Scientific, The Netherlands), using a conversion factor of 5.7. Ash content was determined by AACC official method 08-01 (AACC, 1983). The oil content was determined with a fully automated Büchi extraction system B-811 LSV (Büchi Labortechnik AG, Flawil, Switzerland), using petroleum ether as extraction solvent. The duration of the extraction step was set at 7 hours. The carbohydrates were determined by difference.

\subsubsection{Water holding capacity \& nitrogen solubility}

The water holding capacity (WHC) and nitrogen solubility index (NSI) of the soy protein fractions and full fat flour were determined with a 2 wt.\% dispersion. The dispersions were thoroughly mixed and shaken overnight. Next, the dispersions were centrifuged $(10,000 \mathrm{~g}, 30$ min, $20^{\circ} \mathrm{C}$ ), and the supernatant and pellet were separated. The tubes were drained on tissue paper and the pellets were weighted. Subsequently, the pellet and supernatant were freeze dried (Christ, Germany). The nitrogen content was determined using Dumas analysis (Nitrogen analyser, FlashEA 1112 series, Thermo Scientific, The Netherlands). The WHC was calculated by the ratio of the wet pellet weight over the initial weight of the sample. The NSI was 
determined by the ratio of soluble nitrogen over the total amount of nitrogen initial present in the sample.

\subsubsection{Differential scanning calorimetry}

The protein denaturation temperature and the enthalpy of transition of the soy protein fractions and full fat flour were determined with differential scanning calorimetry (DSC) (Diamond DSC, PerkinElmer, Shelton, USA). The DSC was calibrated with indium, and an empty stainless steel pan was used as reference. The soy protein fractions were dispersed in water ( $20 \mathrm{~g}$ sample/ 100 $\mathrm{g}$ total). The samples were heated from $20{ }^{\circ} \mathrm{C}$ to $150{ }^{\circ} \mathrm{C}$ at $10{ }^{\circ} \mathrm{C} / \mathrm{min}$ and heating cycle was repeated two times. Nitrogen was used as carrier gas. Measurements were analysed with Start Pyris Software (PerkinElmer, Shelton, USA).

\subsubsection{Viscoelastic properties}

The viscoelastic properties of the soy protein fraction and soy protein fraction-full fat flour blends in water were determined with a closed cavity rheometer (CCR, RPA elite, TA instruments, USA). Samples were prepared in a similar way as for structuring experiments (2.2.4). The samples were hydrated in vacuum sealed bags. Subsequently, approximately 5 gram sample was placed between two plastic films and measured in the CCR. An oscillation time sweep was performed at a high frequency $(10 \mathrm{~Hz})$ and strain $(80 \%)$ for $15 \mathrm{~min}$ at $140{ }^{\circ} \mathrm{C}$. A down pressure of 4.5 bar was applied to close the CCR, and therefore prevent water evaporation.

\subsection{Results and Discussion}

In this study, we used aqueous fractionation process to obtain soy protein fractions from toasted and non-toasted soy flour. The developed soy protein fractions were examined on their ability to form fibrous structures when processed in a high temperature shear cell. The most successful samples in fiber formation where further analysed/examined on the most important functional properties with the reverse engineering approach (Otto \& Wood, 1998).

\subsubsection{Aqueous fractionation}

The aqueous fractionation process differs from the conventional fractionation process of soy protein since the organic solvent extraction step is replaced by an aqueous fractionation step. The supernatant, rich in water soluble proteins, was further refined with a protein precipitation step to obtain a soy protein fraction. Yield and composition were determined for fractions obtained with aqueous fractionation from toasted and non-toasted flour (Table 7.1). 
Both soy protein fractions obtained from toasted and non-toasted flour contained still some oil. The fact that some oil remains in the protein fractions was reported before (Campbell et al., 2011). Protein yield from non-toasted soy flour was similar to the yield reported by de Moura, Campbell, De Almeida, Glatz, \& Johnson, 2011. The soy protein fraction obtained from toasted soy flour had a substantially lower yield (12 wt.\% of the total protein present in the initial soy flour) compared to soy protein fraction obtained from non-toasted soy (37 wt.\% of the total protein present in the initial soy flour). In addition, toasting of the soy flour resulted in reduced separation giving a lower protein content. The decrease in protein content and yield was related to decreased solubility of the toasted material (Wu \& Inglett, 1974). In further experiments, we therefore explored the possibility of toasting the soy protein fraction in the final processing step after drying the material, because toasting is normally performed in a dry state.

Table 7.1 Composition analysis and protein yield (percentage of proteins present in soy protein fractions of the initial soy flour) of the soy protein fraction, soy protein concentrate, soy protein isolate (DM=dry matter, a determined by difference, mean value \pm standard deviation $(n=3))$.

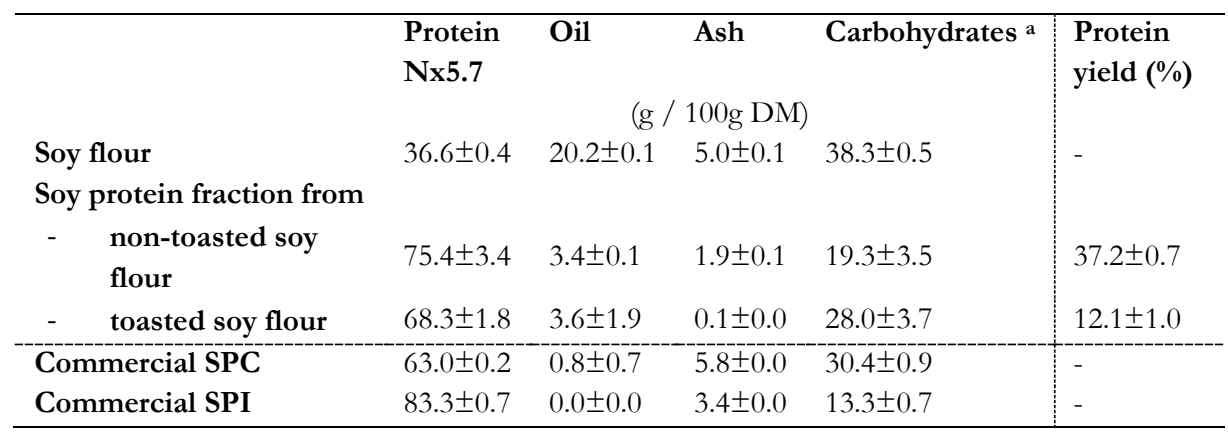

\subsubsection{Shear-induced structuring}

The soy protein fractions (non-toasted and toasted) were structured in the high temperature shear cell (HTSC) to investigate its potential to form fibrous structures. The advantage of using a high temperature shear cell in this study is that relatively small amounts are needed (40 gram). In previous studies, it was shown that fibrous structures were obtained with commercial soy protein concentrate when processing it in a high temperature shear cell, whereas soy protein isolate alone yielded isotropic structures (Grabowska et al., 2016). Therefore, we benchmarked the soy protein fractions against commercial soy protein concentrate and isolate.

Structures obtained with the soy protein fraction only, both toasted and non-toasted, yielded a layered structure (Fig. 7.1A), which was similar to structures that were obtained when soy protein 
isolate was used (Dekkers et al., 2016). Since the soy protein fraction had a higher protein content than commercial soy protein concentrate, we also mixed the soy protein fraction with soy flour in a ratio of 70:30 to obtain a comparable protein content as commercial soy protein concentrate. It must be noted that the main constituent of commercial soy protein concentrate are proteins and polysaccharides, whereas soy flour contains a considerable amount of fat as well. Hence by mixing soy protein fraction with soy flour, we incorporated fat. Nevertheless, similar fibrous structures were obtained. The non-toasted blend resulted in an isotropic gel (Fig. 7.1B), whereas toasted $\left(\right.$ at $150^{\circ} \mathrm{C}$ ) soy protein fraction / soy flour blend yielded distinct fibrous structures (Fig. 7.1C). These structures are similar to soy protein concentrate presented in literature (Grabowska et al., 2016). Toasting the soy protein fraction / soy flour blend at higher temperatures (175-200 $\left.{ }^{\circ} \mathrm{C}\right)$ resulted in incoherent and brittle structures, without any fibrous appearance.

A

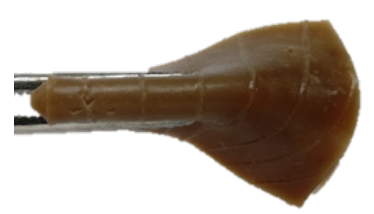

$\mathrm{B}$

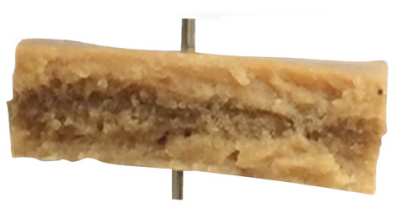

C

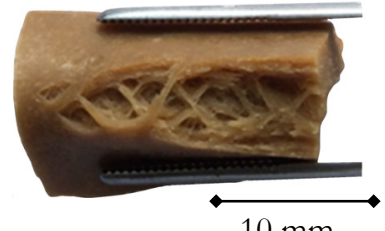

Fig. 7.1 Visual appearance of structured samples made with A) $44 \mathrm{wt} . \%$ non-toasted soy protein fraction B) $44 \mathrm{wt} . \%$ nontoasted soy protein fraction / full fat flour blend (70/30), and C) $44 \mathrm{wt} . \%$ toasted soy protein fraction / full fat flour blend (70/30).

Fig. 7.2 shows the results of the tensile strength analysis of structures in which we compare soy protein fraction alone with the soy protein fraction mixed with soy flour, both toasted and nontoasted. We compare these results with commercial soy protein isolate and concentrate. The tensile stress and strain differences between parallel and perpendicular were small for the toasted and non-toasted soy protein fraction alone, resulting in a low anisotropic indices $\left(\mathrm{AI}_{\sigma} / \mathrm{AI}_{\varepsilon}=1.13\right.$ - 1.35). Blending non-toasted soy protein fraction with full fat flour resulted in a decrease of the tensile stress and strain, resulting in even lower anisotropic indices $\left(\mathrm{AI}_{\sigma} / \mathrm{AI}_{\varepsilon}=0.97-1.14\right)$. The toasted soy protein fraction / soy flour blends resulted in a large difference in tensile stress and strain for samples taken in parallel and perpendicular, and hence in a higher anisotropic index $\left(\mathrm{AI}_{\sigma} / \mathrm{AI}_{\varepsilon}=1.77\right.$ - 1.88). This corresponds to the visual fibrous appearance of this sample. The effect of blending the soy protein fraction with full fat flour had little effect on the Young's modulus and also toasting hardly affected the Young's modules. The anisotropic index of the toasted soy protein fraction / full fat flour blend was higher compared to commercial soy protein concentrate. The absolute tensile stress and strain of the soy protein fractions/soy flour blend were in between the strength of soy protein concentrate and soy protein isolate. Hence, the 
anisotropic indices of the samples prepared with the fractions obtained with an aqueous fractionation process were similar than those obtained with commercial soy proteins concentrates. Similar tensile strength analysis were performed on beef as reported by Krintiras et al.; the tensile strength of the fibrous structures prepared with the toasted soy protein fraction / full fat flour blend were similar values reported for raw beef (Krintiras et al., 2014).
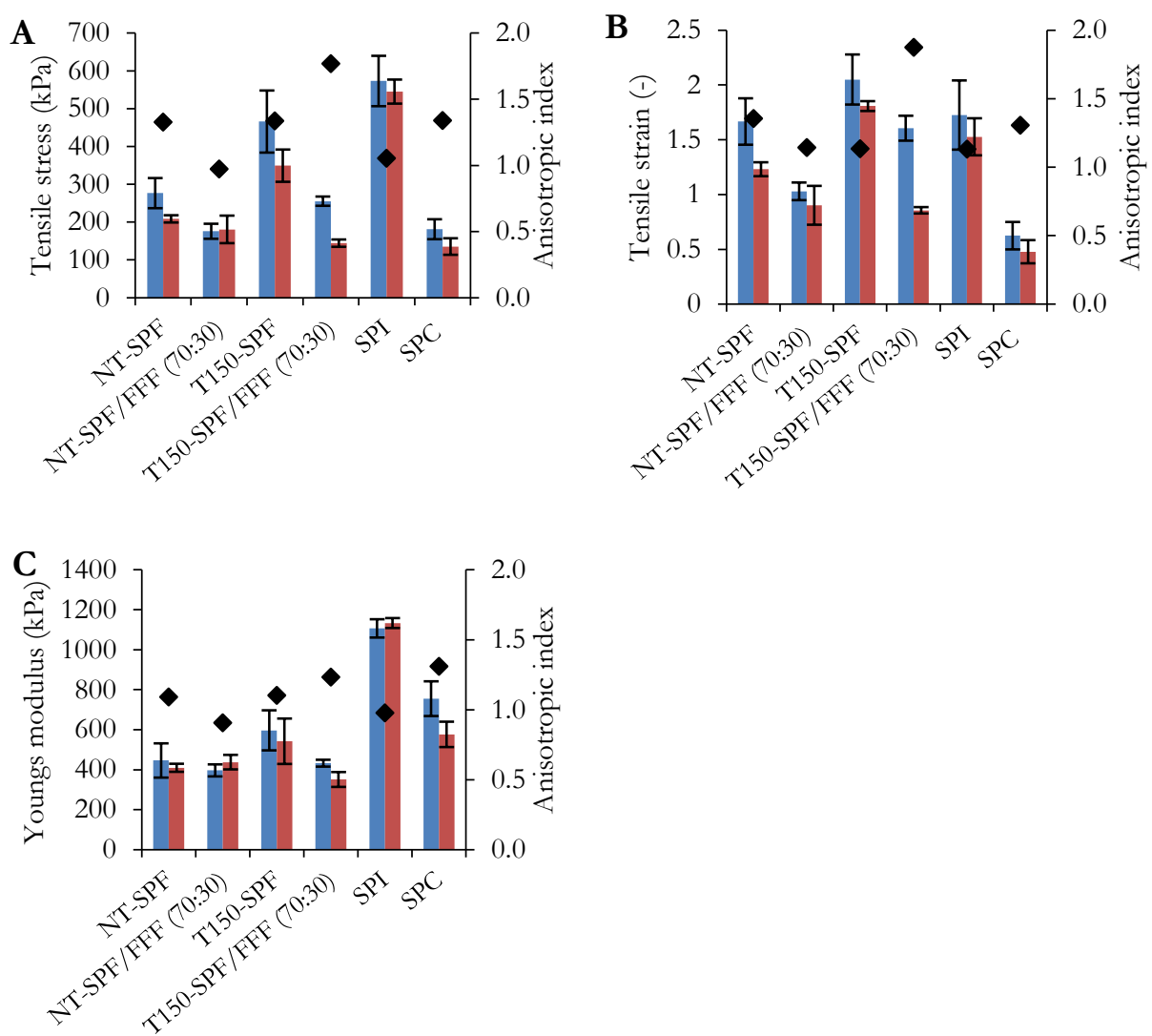

Fig. 7.2 Tensile strength analysis results of non-toasted (NT) soy protein fraction (SPF) and SPF / full fat flour (FFF) blend in the ratio $70 / 30$, and toasted at $150{ }^{\circ} \mathrm{C}(T)$ soy protein fraction (SPF) / full fat flour (FFF) blend (70/30), commercial soy protein isolate (SPI), soy protein concentrate (SPC), A) tensile stress B) tensile strain C) Young's modulus (blue $=$ samples in parallel direction, red $=$ samples in perpendicular direction, black diamonds $=$ anisotropic index, mean value \pm absolute deviation $(n=2)$. 


\subsubsection{Functional properties of soy protein fractions}

\subsubsection{Water holding and nitrogen solubility}

Fibrous structure formation was positively influenced by toasting the dried soy protein fraction at $150{ }^{\circ} \mathrm{C}$. The differences between the soy protein fractions due to toasting became apparent when adding water (Fig. 7.3). Hydration of non-toasted, toasted at 50 and $100{ }^{\circ} \mathrm{C}$ soy protein fractions were well dispersible in water and partly dissolved resulting in a paste. Toasting the soy protein fraction at 125 and $150{ }^{\circ} \mathrm{C}$ absorbed water, leading to a crumbly texture, which was comparable to commercial soy protein isolate and concentrate. The aqueous soy protein fractions were slightly yellowish. These differences can be related to differences in composition, such as the presence of oil or carbohydrates, and the differences in fractionation processes. Toasting the soy protein fraction at 175 and $200{ }^{\circ} \mathrm{C}$ resulted in a sandy structure, with a dark brown colour and a burned smell indicating that at these high temperatures the samples started to degrade.
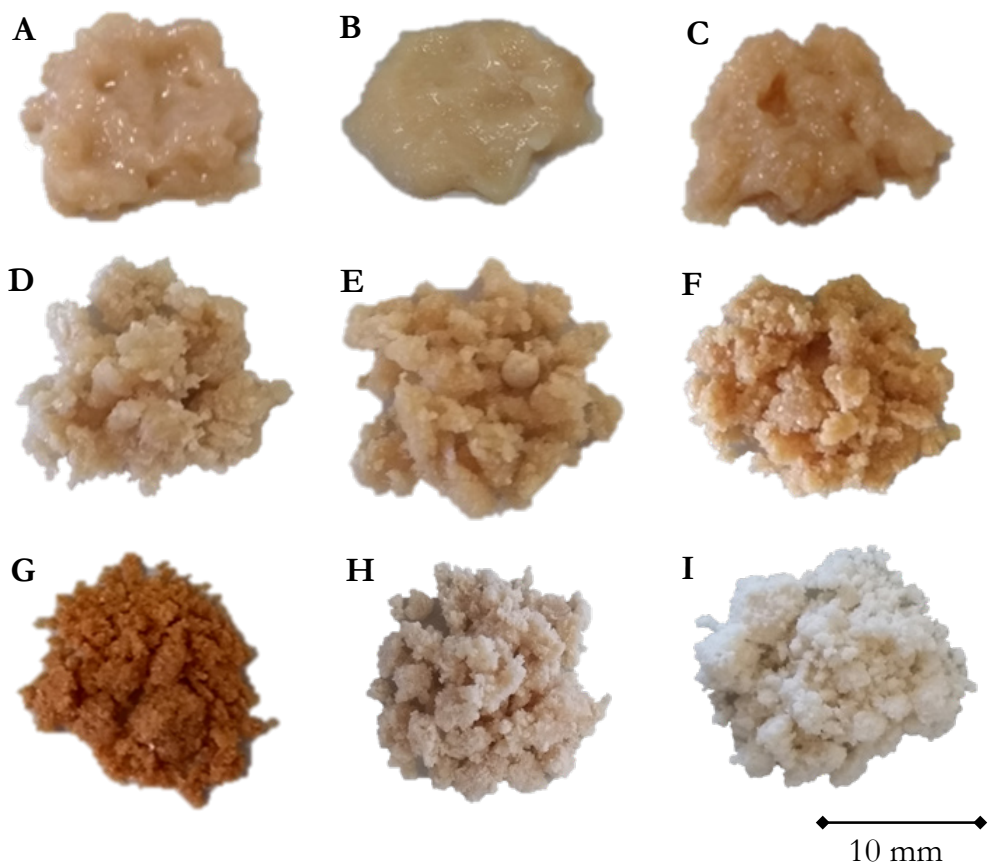

Fig. $7.340 \mathrm{wt} . \%$ dispersion of non-toasted soy protein fraction (A) and the soy protein fractions toasted at $50{ }^{\circ} \mathrm{C}(\mathrm{B}), 100$ ${ }^{\circ} \mathrm{C}(\mathrm{C}), 125^{\circ} \mathrm{C}(\mathrm{D}), 150^{\circ} \mathrm{C}(\mathrm{E}), 175^{\circ} \mathrm{C}(\mathrm{F}), 200^{\circ} \mathrm{C}(\mathrm{G})$, and the commercial soy protein isolate (H) and soy protein concentrate (I), sample size was approximately $15 \mathrm{~mm}$.

The differences in hydration properties of the soy protein fractions were quantified by measuring the water holding capacity (WHC) and the nitrogen solubility index (NSI) of the soy protein 
fractions. In Fig. 7.4, the WHC and NSI are shown of the soy protein fractions non-toasted and toasted at different temperatures $\left(50-200^{\circ} \mathrm{C}\right)$. The WHC increased with toasting temperature up to $150^{\circ} \mathrm{C}$. A further increase of the toasting temperature led to a decrease in WHC. The WHC of the soy protein fraction toasted at $150{ }^{\circ} \mathrm{C}$ was in between the values for SPI and SPC. A relatively high WHC seems important for fibrous structure formation.
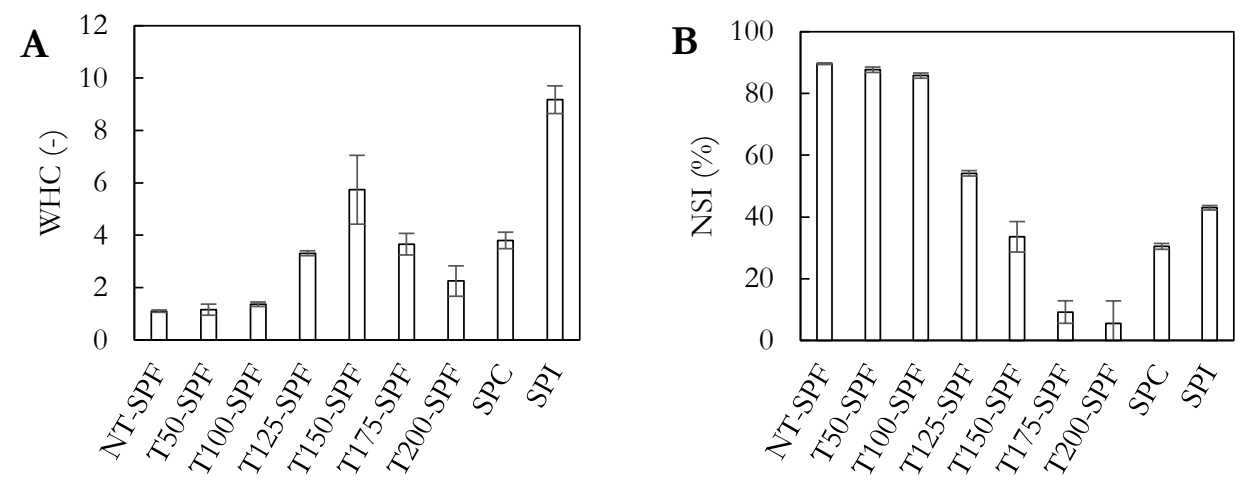

Fig. 7.4 A water bolding capacity (WHC) and B) nitrogen solubility index (NSI)) for denatured soy protein fraction nontoasted (NT-SPF) and toasted at various temperatures (50, 100, 125, 150, 175 and $200{ }^{\circ} \mathrm{C}$ ) soy protein fractions (T..$S P F)$, and the commercial soy protein concentrate and isolate (SPC and SPI) $(n=3)$.

Toasting also resulted in a lower NSI. The commercial soy protein isolate and concentrate had overall a low NSI, which is comparable to the NSI of the soy protein fraction toasted at $150{ }^{\circ} \mathrm{C}$. The results suggest that full solubility is not a requirement, or possibly even a disadvantage when the fractions is used to make anisotropic materials.

Overall, the commercial samples as well as the soy protein fraction toasted at $150^{\circ} \mathrm{C}$ formed upon hydration a crumbly texture, which was assumed to be related water absorption by the protein particles. At lower toasting temperatures, the soy protein fraction formed a sticky texture, which could probably related to the higher NSI and subsequently water was mostly bound interstitially. At higher toasting temperatures, the soy protein fraction formed a sandy structure because almost no proteins were dissolved, and hence there was limited interstitial water present (Peters, Vergeldt, Boom, \& van der Goot, 2017). Previous studies also showed that toasting or a heat treatment resulted in lowering the protein solubility (Nagmani \& Prakash, 1997; Narayana \& Narasinga Rao, 1982; Petruccelli \& Añón, 1994; Wu \& Inglett, 1974). A decrease in solubility in thermally treated proteins is often linked to denaturation (Lopez de Ogara, Bercovich, Pilosof, \& Bartholomai, 1986) and possibly protein aggregation (Lopez de Ogara et al., 1986; Narayana \& Narasinga Rao, 1982). 


\subsubsection{State of the proteins}

The denaturation temperatures $\left(T_{d}\right)$ and enthalpy of transition $(\Delta H)$ of the toasted and nontoasted soy protein fractions was determined to determine whether protein denaturation is indeed causing the differences in solubility (Table 7.2). The commercial soy protein isolate and concentrate sample did not show any peaks, which indicated that most proteins were already denatured during the conventional fractionation process. The soy protein fractions toasted up to $150{ }^{\circ} \mathrm{C}$ showed two peaks, around $77^{\circ} \mathrm{C}$ and $95^{\circ} \mathrm{C}$, indicating that aqueous fractionation did not result in complete denaturation of the proteins. The two peaks can be related to the denaturation of the two major storage proteins present, conglycinin and glycinin (A. M. Hermansson, 1986). Yet, the enthalpy of transition $(\Delta H)$ slightly decreases with an increasing dry-toasting temperature, indicating that there are transitions occurring in the soy protein fraction. The toasting step was performed in a dry state, which limited the denaturation even at high temperatures (Wu \& Inglett, 1974). Though, when the soy protein fraction was toasted at a temperature of 175 or $200{ }^{\circ} \mathrm{C}$, no peaks could be identified, which indicated that the sample was mostly denatured. It was already observed that toasting at these temperatures resulted in degradation. Based on the results above, we can conclude that it is not necessary for proteins to be native for successful transformation into fibrous structures. This hypothesis is further supported by the fact that additional experiments showed that heating the soy protein fraction in a wet state resulted in denaturation. Nevertheless, shear-induced structure formation with denatured soy protein fraction / full fat flour blends resulted in isotropic structures similar to non-toasted blends (supplementary information).

Table 7.2 Endothermal differential scanning calorimetry (DSC) peaks (20 wt.\% dry matter, heating rate $\left.\left.10^{\circ} \mathrm{C} / \mathrm{min}, n=2\right)\right)$ of several soy protein fraction, and commercial soy protein isolate and concentrate $(N D=$ not detected)

\begin{tabular}{|c|c|c|c|c|}
\hline & Peak 1 & & Peak 2 & \\
\hline & $\mathbf{T}_{\mathrm{d}}\left({ }^{\circ} \mathrm{C}\right)$ & $\Delta \mathbf{H}(\mathrm{J} / \mathrm{g})$ & $\mathbf{T}_{\mathrm{d}}\left({ }^{\circ} \mathrm{C}\right)$ & $\Delta \mathbf{H}(\mathrm{J} / \mathrm{g})$ \\
\hline \multicolumn{5}{|c|}{ Soy protein fraction } \\
\hline Non-toasted & $77.9 \pm 0.5$ & $0.22 \pm 0.03$ & $96.1 \pm 0.0$ & $0.79 \pm 0.01$ \\
\hline $50^{\circ} \mathrm{C}$ & $78.0 \pm 0.0$ & $0.28 \pm 0.02$ & $95.1 \pm 0.0$ & $0.91 \pm 0.09$ \\
\hline $100^{\circ} \mathrm{C}$ & $78.0 \pm 0.1$ & $0.27 \pm 0.03$ & $95.3 \pm 0.1$ & $0.86 \pm 0.05$ \\
\hline $125^{\circ} \mathrm{C}$ & $78.0 \pm 0.7$ & $0.17 \pm 0.03$ & $95.4 \pm 0.3$ & $0.78 \pm 0.01$ \\
\hline $150^{\circ} \mathrm{C}$ & $77.6 \pm 0.0$ & $0.19 \pm 0.01$ & $95.4 \pm 0.3$ & $0.78 \pm 0.01$ \\
\hline $175^{\circ} \mathrm{C}$ & ND* & ND & ND & ND \\
\hline $200^{\circ} \mathrm{C}$ & $\mathrm{ND}^{*}$ & ND & ND & ND \\
\hline Commercial SPC & ND & ND & $\mathrm{ND}$ & ND \\
\hline Commercial SPI & ND & ND & ND & ND \\
\hline
\end{tabular}




\subsubsection{Viscoelastic properties}

The viscoelastic properties of the soy protein fraction toasted at various temperatures were determined during a high frequency and high strain treatment in a closed cavity rheometer at $140{ }^{\circ} \mathrm{C}$. These conditions were used to mimic the shear process as applied in the high temperature shear cell. Fig. 7.5A compares the complex modulus $\left(G^{*}\right)$ of non-toasted and toasted soy protein fraction at various temperatures. Three clusters in $G^{*}$ were observed when toasting the soy protein fraction; $50-100{ }^{\circ} \mathrm{C}, 125-150{ }^{\circ} \mathrm{C}$, and $175-200{ }^{\circ} \mathrm{C}$. These clusters were also found when hydrating the soy protein fraction (7.3.3.1). It can be seen that toasting at $125-150{ }^{\circ} \mathrm{C}$ increased the $G^{*}$ with roughly one order of magnitude $(1-10 \mathrm{kPa})$ as compared to non-toasted, resulting in a $G^{*}$ more similar to the commercial concentrate and isolate (Fig. 7.5B). This toasting temperature was found to be optimal for shear-induced structuring. Toasting at even higher temperatures $\left(175-200{ }^{\circ} \mathrm{C}\right)$ increased the $G^{*}$ even further, however this toasting temperature resulted in incoherent, crumbly structures. The initial bump found in the toasted soy protein fraction up to a toasting temperature of $150{ }^{\circ} \mathrm{C}$ might be related to denaturation of the proteins, since all samples showing this bump also showed two endothermic peaks. In addition, the viscoelastic properties of the soy protein fraction / full fat flour blend were determined and showed similar results (data shown supplementary information).
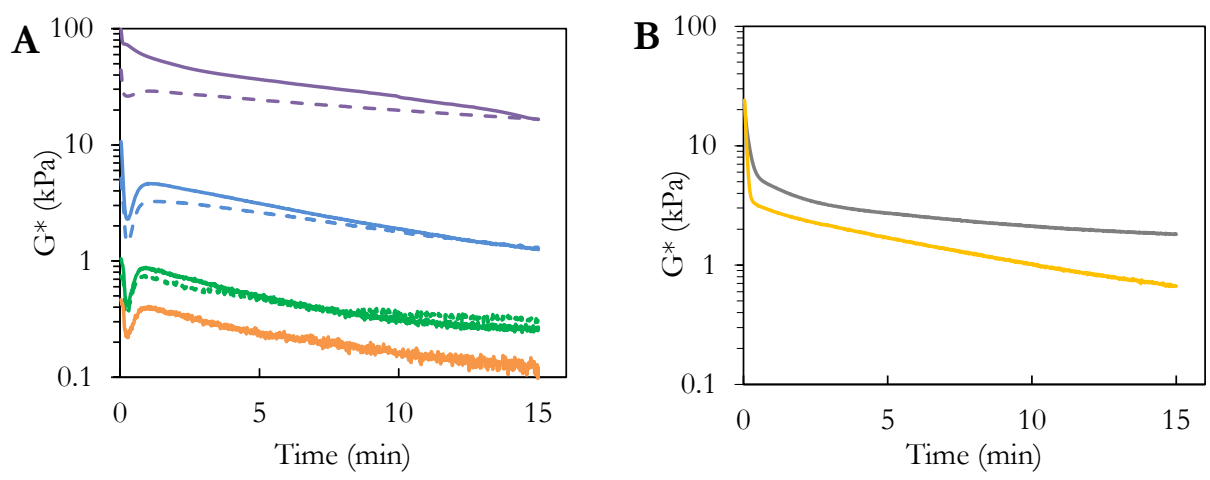

Fig. 7.5 Apparent complex modulus $\left(G^{*}\right)$ measured during a time-sweep experiment at high strain $(80 \%)$ and high frequency $(10 \mathrm{~Hz})$ at $140^{\circ} \mathrm{C}$ of A) $44 \mathrm{wt}$.\% soy protein fraction non-toasted $(-)$ and toasted at $50^{\circ} \mathrm{C}(\ldots), 100{ }^{\circ} \mathrm{C}(-), 125$ ${ }^{\circ} \mathrm{C}(\ldots), 150^{\circ} \mathrm{C}(-), 175^{\circ} \mathrm{C}(\ldots), 200^{\circ} \mathrm{C}(-)$, and B) $44 \mathrm{wt} \%$ commercial soy protein isolate $(-)$ and soy protein concentrate $(-)(n=3)$. 


\subsubsection{Design of functional properties}

A reversed engineering approach was used to design the fractionation process aimed at obtaining functional properties needed for making fibrous structures. The results in this paper indicate that the functional properties needed alter during fractionation and structuring and that relevant functional properties do not always coincide with properties generally considered. When having the application of meat analogues in mind, it is not necessary to focus on purity optimization, and on classical functional properties such as high solubility. In this study, high water holding and medium nitrogen solubility seem more relevant for fibrous structure formation. With the use of a reversed engineering approach, we therefore adapted the conventional processes (i.e. the organic solvent extraction was exchanged with an aqueous extraction step and a toasting step was applied later) and designed an aqueous fractionation process tailored to yield fibrous structures.

In addition, we used minimal and mild fractionated ingredients as starting material to make fibrous structures. Sturtewagen et al. (2016) and Apaiah et al. (2006) indicated that the production of meat analogues is currently not much more efficient than pork meat (Apaiah, Linnemann, \& Van Der Kooi, 2006; Sturtewagen et al., 2016). This is mainly due to the intensive ingredient preparation of conventional ingredients. It would therefore be beneficial if minimal and mild fractionated ingredients can be used in meat analogues, thereby making a significant contribution in respect to sustainability.

\subsection{Conclusions}

With a reverse engineering approach, we gained important insights in the essential functional properties of soy protein fractions that are required to obtain fibrous protein structure, which could form the basis for a next generation meat analogues. The essential functional properties of the fractions were obtained by changing process steps. In this study, it was shown that toasting is an important processing step to obtain the functional properties needed for fibrous structure formation. The toasting temperature altered the functionality of the soy protein fractions, and three toasting temperature clusters could be distinguished. The functional properties of the three clusters revealed that soy protein fractions with a relatively high water holding capacity, intermediate nitrogen solubility and viscoelastic properties characterized as $G^{*}$ between $1-10$ $\mathrm{kPa}$ were shown to lead to fibrous structures. Most distinct fibrous structures were obtained with a mixture of $70 \mathrm{wt} . \%$ toasted $\left(150^{\circ} \mathrm{C}\right)$ soy protein fraction and $30 \mathrm{wt} . \%$ native soy flour. 
Toasting early in the fractionation process impacted the yield negatively, since it decreased the solubility. High protein solubility was necessary during protein fractionation to obtain a high yield. Hence, the toasting step is recommended to be applied after aqueous fractionation due to the differences in functional properties needed during fractionation and structuring.

Furthermore, the most distinct fibrous structures in this study were formed with a mixture of more intensive and limited fractionated ingredients. This combination is an interesting option for product development. Forthcoming studies could compare this combination of intensive and limited fractionation with conventional fractionation to determine whether this design is a preferred route for producing more sustainable foods in the future.

\section{Acknowledgements}

The authors wish to thank Ilse Wubs, Sharifah Ramli, and Remco de Lange for their contributions in experimental work. The project is partly financially supported by the institute for sustainable technology (ISPT), the Netherlands. 


\section{Supplementary information}

Table S1 Tensile strength analysis of denatured and denatured and toasted $\left(150^{\circ} \mathrm{C}\right)$ soy protein fraction (SPF) / full fat flour (FFF) blends (70:30).

\begin{tabular}{lccc}
\hline & & $\begin{array}{c}\text { Tensile stress } \\
(\mathrm{kPa})\end{array}$ & $\begin{array}{c}\text { Tensile strain } \\
(-)\end{array}$ \\
\hline Denatured SPF/FFF (70:30) & Par & $140 \pm 21$ & $0.89 \pm 0.05$ \\
& Per & $92 \pm 16$ & $0.51 \pm 0.04$ \\
Denatured \& Toasted 150-SPF/FFF (70:30) & AI & 1.53 & 1.73 \\
& Par & $329 \pm 61$ & $2.10 \pm 0.20$ \\
& Per & $126 \pm 11$ & $0.83 \pm 0.03$ \\
& AI & 2.61 & 2.53 \\
\hline
\end{tabular}

Table S2 Water holding capacity (WHC), nitrogen solubility index (NSI), denaturation temperature (Td) and enthalpy of transition $(\Delta H)$ of denatured and toasted $\left(150^{\circ} \mathrm{C}\right)$ soy protein fraction $(S P F)(N D=$ not detected).

\begin{tabular}{lcccc}
\hline & $\begin{array}{c}\text { WHC } \\
(-)\end{array}$ & $\begin{array}{c}\text { NSI } \\
(\%)\end{array}$ & $\begin{array}{c}\mathrm{T}_{\mathrm{d}} \\
\left({ }^{\circ} \mathrm{C}\right)\end{array}$ & $\begin{array}{c}\Delta \mathrm{H} \\
(\mathrm{J} / \mathrm{g})\end{array}$ \\
\hline Denatured SPF & $0.29 \pm 0.01$ & $95.4 \pm 0.9$ & $\mathrm{ND}$ & $\mathrm{ND}$ \\
Denatured \& Toasted 150-SPF & $7.14 \pm 0.66$ & $11.4 \pm 3.3$ & $\mathrm{ND}$ & $\mathrm{ND}$ \\
\hline
\end{tabular}

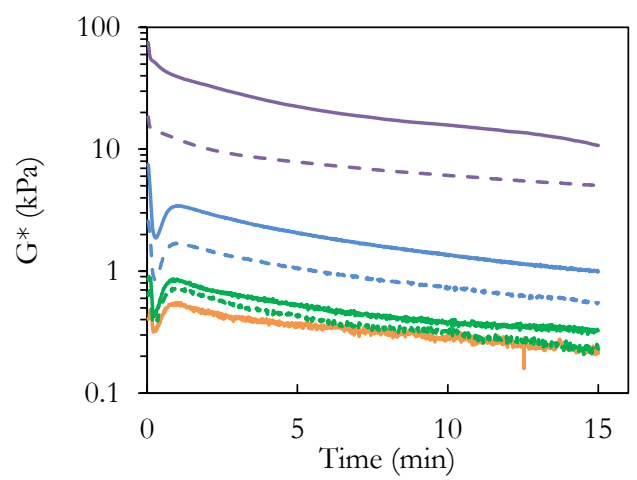

Figure $S 1$ Apparent complex modulus $\left(G^{*}\right)$ measured during a time-sweep experiment at bigh strain (80\%) and bigh frequency $(10 \mathrm{~Hz})$ at $140^{\circ} \mathrm{C}$ of $\left.\mathrm{A}\right) 44 \mathrm{wt} . \%$ soy protein fraction / full fat flour blend $(70 / 30)$ non-toasted ( - ) and toasted at $50{ }^{\circ} \mathrm{C}(\ldots), 100{ }^{\circ} \mathrm{C}(-), 125^{\circ} \mathrm{C}(\ldots), 150{ }^{\circ} \mathrm{C}(-), 175^{\circ} \mathrm{C}(\ldots), 200{ }^{\circ} \mathrm{C}(-)$ 


\section{Chapter 8}

Structuring processes for fibrous plant protein products

This chapter with some adaptations is submitted as B.L. Dekkers, R.M. Boom, A.J. van der Goot (2018) Structuring processes for meat analogues. 


\section{Abstract}

Background: Animal derived proteins foods, such as meat, have a large impact on the environment. Meat analogues are products that replace meat in its functionality, i.e. have similar product properties and sensory attributes as meat, which is achieved by the fibrous nature of those products. The techniques used to make fibrous products are outlined in this review paper.

Scope and approach: Structuring techniques are compared in their approach, being either bottomup, which refers to assembly of structural elements that are combined, or top-down, which refers to structuring of biopolymer blends using an overall force field. The strengths and weaknesses of these techniques are discussed in terms of (achievable) product resemblance, robustness, scalability, and resource efficiency. The fibrous structures created with techniques with a topdown strategy are subsequently further outlined.

Key findings and conclusions: Strengths and weaknesses can be found for both the bottom-up and top-down strategy. The bottom-up strategy has the potential to resemble the structure of meat most closely, by structuring the proteins hierarchically through assembly of individual structural components. The top-down strategy is better scalable, is more efficient in its use of resources, but can only create the desired structure on larger length scales. While until now most analysis methods focus on the (micro)structural anisotropy of the resulting fibrous products, there is a clear need for methods that would allow the in situ analysis of the evolution of the structure during the structuring process. 


\subsection{Introduction}

The production of protein food should be significantly increased to accommodate the growth of the world population and its affluence in the coming decades. However, animal derived products and especially meat have a large impact on the environment in terms of water and land use, greenhouse gas emission, and energy consumption (Steinfeld, Gerber, Wassenaar, Castel, \& de Haan, 2006). A disproportionate amount of plant protein is required for the production of meat. On average, $6 \mathrm{~kg}$ of plant protein is required for $1 \mathrm{~kg}$ of meat protein (Aiking, 2011; Pimentel, 2003). The amount of feed required varies between 3.3, 6.4 and $25 \mathrm{~kg}$ for poultry, pork or beef respectively (Alexander, Brown, Arneth, Finnigan, \& Rounsevell, 2016). Besides sustainability issues, also ethical aspects with regards to animal welfare and health concerns are factors in the discussion that meat consumption should be reduced (Clark, Stewart, Panzone, Kyriazakis, \& Frewer, 2017; McAfee et al., 2010; Micha, Wallace, \& Mozaffarian, 2010). Although consumers are increasingly aware of the environmental burden, animal welfare issues and the health concerns that are associated with meat, so far only a fraction of the world population chooses a vegetarian diet (Stiftung, Böll, \& Terre, 2014). Most people consume meat, because it is perceived as tasty, healthy and nutritious, and it is deeply embedded in many cultures (Schösler, de Boer, \& Boersema, 2012; Verbeke et al., 2010). To help consumers reduce their meat consumption, several strategies have been developed:

- One strategy is encouragement of 'meatless days' or smaller portion sizes (Laestadius, Neff, Barry, \& Frattaroli, 2013). This approach recognizes that many people overconsume proteins. This approach may be beneficial for resource use and for health, as long as it is embraced by the right group of consumers, however it can only reduce meat consumption by a modest degree.

- As a second strategy, meat in a meal can be replaced either by the consumption of vegetables, beans, pulses, and/or nuts (Day, 2013; De Boer, Schösler, \& Aiking, 2014).

- A third strategy is based on the development of texturized products. Tofu and tempeh are examples of texturized products, which have been consumed in east Asia for centuries. However, the acceptance of these types of products is lower in Western countries (Asgar, Fazilah, Huda, Bhat, \& Karim, 2010). Therefore another category of texturized products has emerged, referred to as meat analogues. Meat analogues are products that can replace meat in its functionality, being similar in product properties/sensory attributes, and that 
can also be prepared by consumers as if they were meat. The resemblance of these products to meat, in terms of texture, taste, appearance, and smell, is important for consumers that currently mostly choose meat (Hoek et al., 2011).

In this review, we outline techniques to create fibrous plant protein materials that can be used to develop meat analogues. We classify these techniques by their fundamental approach to create this fibrous structure of meat muscles. Some techniques adopt a bottom-up strategy in which anisotropic structural elements are created that are subsequently assembled into larger products. Other techniques create fibrous products by structuring biopolymer blends via the top-down strategy in which the fibrousness of the product becomes apparent when stretching the material, mimicking the structure on larger length scales only. The strengths and weaknesses of these techniques are compared in terms of product quality, process robustness, scalability and environmental impact. The techniques with the top-down strategy are further contextualized by relating them with production processes using polymer or biopolymer blends aimed at other purposes than creating fibrous products. These insights are subsequently translated to fibrous protein structuring. Finally, we discuss the analytical methods to quantify the (micro)structure of products after processing.

\subsection{Structuring techniques}

In this section, we discuss the different processing techniques that can be followed to produce fibrous products. We describe the established techniques that already operate on commercial scale, and novel techniques that are less mature.

\subsubsection{Established processing techniques}

\subsubsection{Extrusion}

Extrusion is the most commonly used commercial technique to transform plant-based materials into fibrous products. We distinguish two classes of structuring with extrusion: low-moisture and high-moisture. In low-moisture extrusion, flours or concentrates are mechanically processed into texturized vegetable proteins (TVP), which are dry, slightly expanded products that are moisturized afterwards. In high moisture extrusion, fibrous products are produced with moisture contents above $50 \mathrm{wt} . \%$. The proteins are plasticized/molten inside the barrel by a combination of heating, hydration and mechanical deformation. When this protein-'melt' flows into the die, it gets aligned by the (inhomogeneous) laminar flow and is cooled to prevent expansion. High moisture extrusion processes were extensively studied in the eighties and nineties (Arêas, 1992; 138 
Cheftel, Kitagawa, \& Queguiner, 1992; Mitchell \& Areas, 1992). Mitchell and Areâs proposed the 'suspension model' to mechanistically explain extrusion texturization (Mitchell \& Areas, 1992). According to this model, the biopolymer melt forms two phases; a homogenous continuous phase and a dispersed, insoluble phase. The insoluble, dispersed phase is either formed during processing in the barrel at high temperatures, or was already present in the raw materials prior to processing. Whether materials/ingredients can be extruded depends on the ratio of soluble and insoluble components; too many insoluble components disturb protein cross-linking and result in incoherent products. While extrusion is the most widely applied technology for the product of meat analogues, it is relatively energy intensive and, being a very complex process, does not offer very detailed control over the final morphology.

\subsubsection{Mycoprotein}

The filamentous fungus Fusarium venenatum is used since the mid-1980s as a basis for meat analogues that are marketed under the brand name Quorn. The fungus is produced in a continuous fermentation process in bioreactors. The conditions in the bioreactor are critical for the production of the fungus, for example temperature and $\mathrm{pH}$ should be monitored and strictly controlled. After fermentation, the RNA has to be degraded into monomers by a heat treatment, so it can diffuse out of the cells. The residual biomass is heated and centrifuged to obtain a pastelike product with 20 wt.\% solids (Wiebe, 2002; Wiebe, 2004). The filamentous fungus is unordered after this centrifugation step, and therefore further process steps are required to obtain a fibrous product. In practice, further steps, such as forming, steaming, chilling, and texturizing, are required to obtain fibrous products. Minced-type products, such as chunks, sausages, and burgers, are commercially available from this material (Wiebe, 2002). While this route has been a commercial success for decades, the process is relatively intensive in its use of resources, and high in energy usage in the process and ingredient production (Smetana, Mathys, Knoch, \& Heinz, 2015).

\subsubsection{Mixing of proteins and hydrocolloids}

Fibrous products can also be obtained by mixing protein with hydrocolloids that precipitate with multivalent cations (Kweldam, 2011). After mixing, the fibrous products are washed and the excess water is removed by pressing, yielding dry matter contents between 40 - 60 wt.\%. Despite the initial ordering in the shear direction, the subsequent steps destroy this large range ordering, limiting the use to minced meat products, such as burgers and schnitzels. In this process, various 
combinations of proteins, hydrocolloids and multivalent cations can be used. For example, a product based on casein and alginate was introduced in 2005 under the brand name Valess. Plant proteins such as soy, rice, maize and lupine can be employed in a similar way. This process is well scalable, yields product with some degree of structure, but still is relatively intensive in its use of resources.

\subsubsection{Novel processing techniques}

\subsubsection{Freeze structuring}

In freeze structuring or freeze alignment, an aqueous solution or slurry of proteins is frozen to generate structure. Heat removal from a well-mixed slurry leads to an isotropic structure, but when heat is removed unidirectionally without mixing, then alignment of ice crystal needles yields anisotropic structure. Directional freezing has been studied for structuring of meat, fish, and plant proteins (Consolacion \& Jelen, 1986; Lugay \& Kim, 1978; Middendorf, Waggle, \& Cornell, 1975), but is also employed for the creation of porous metal and ceramic materials. The size of the ice crystal needles can be tailored with the freezing temperature and rate (Lugay \& Kim, 1978). Subsequently, the frozen product is dried without melting the ice crystals, for example by freeze drying, to obtain a porous microstructure with sheet-like parallel orientation of the proteins. These sheets are connected forming a cohesive fibrous product (Consolacion \& Jelen, 1986). To obtain distinct fibrous products, the proteins should have relatively good solubility prior to freezing, and during the freezing process these proteins become insoluble (Lugay \& Kim, 1978).

\subsubsection{Shear cell technology}

Based on the recognition that extrusion is an effective, but not a well-defined process, a technology based on well-defined shear flow deformation was introduced a decade ago to produce fibrous products (Manski, van der Goot, \& Boom, 2007b). Shearing devices inspired on the design of rheometers (Manski, van der Goot, \& Boom, 2007a), so-called shear cells, were developed in which intensive shear can be applied in a cone-in-cone or in a couette geometry (Krintiras, Göbel, Bouwman, van der Goot, \& Stefanidis, 2014; Van den Einde et al., 2004). The final structure obtained with this technique depends on the ingredients and on the processing conditions. Fibrous products are obtained with calcium caseinate and several plant protein blends, such as soy protein concentrate, soy protein isolate (SPI) - wheat gluten (WG), and SPI - pectin (Dekkers, Nikiforidis, \& Goot, 2016; Grabowska et al., 2016; Grabowska, Tekidou, Boom, \& van der Goot, 2014; Manski et al., 2007b). The structures prepared with calcium 140 
caseinate showed anisotropy on a nanoscale, while for the plant-based material, anisotropy was observed up to the micrometre-scale. The technology was successful up to pilot scale (Krintiras, Gadea Diaz, van der Goot, Stankiewicz, \& Stefanidis, 2016).

\subsubsection{Wet spinning}

Boyer patented wet spinning of proteins for the application of meat analogues in 1954 (Boyer, 1954). A solution containing protein is extruded through a spinneret, and subsequently immersed into a bath containing a non-solvent for the protein. Exchange of solvent and non-solvent results in precipitation and solidification of the extruded protein phase, forming stretched filaments with a thickness in the order of $20 \mu \mathrm{m}$ (Boyer, 1954; Rampon, Robert, Nicolas, \& Dufour, 1999; Tolstoguzov, 1988). Several studies showed the food-grade production of fibres with plant-based materials such as soy, pea and fababean (Culioli \& Sale, 1981; Gallant, Bouchet, \& Culioli, 1984; Rampon et al., 1999). The type of structure that is formed depends on the solidification mechanism: fibres are obtained when the dispersed phase solidifies and the continuous phase can be washed away; capillary filled gels are obtained when the continuous phase is solidified and the dispersed phase stays liquid, and fibre filled gels are obtained when both the dispersed and the continuous are solidified (Tolstoguzov, 1988). Wet spinning is mostly used for the creation of individual fibres, and is one of the standard techniques for the production of membranes for industrial separation purposes (Ho \& Sirkar, 2012).

\subsubsection{Electrospinning}

In electrospinning, a biopolymer solution is pushed through a hollow needle or spinneret that has an electric potential relative to a ground electrode. Accumulation of charge on the surface of the droplet that emerges from the spinneret, causes surface instabilities that ultimately grow into very thin fibres $(\approx 100 \mathrm{~nm}$ ), which are attracted to the ground electrode (Schiffman \& Schauer, 2008). Electrospinning of food-grade biopolymers has received increased attention lately (Ghorani \& Tucker, 2015; Schiffman \& Schauer, 2008). Food-grade electrospinning was mostly presented for applications in which nanofibers were used as carriers or delivery systems for bioactives components, such as polyphenols and probiotics (Librán, Castro, \& Lagaron, 2017), but electrospinning can also be used to produce fibres for the application of meat analogues (Nieuwland et al., 2014). Electrospinning of proteins has been reported for several animal-based proteins, such as whey, collagen, egg and gelatin, but only sparingly for plant proteins (Anu Bhushani \& Anandharamakrishnan, 2014; Ghorani \& Tucker, 2015). Zein, when 
dissolved in a $80 \mathrm{wt} \%$ ethanol solution, was spun into fibres with a thickness in the nanoscale (Miyoshi, Toyohara, \& Minematsu, 2005). Nieuwland et al. showed the possibility to use zein as a carrier for other proteins, such as soy protein (Nieuwland et al., 2014). The proteins are required to be highly soluble, and behave a like random coil instead of globulins. Those requirements not generally met by plant proteins, since in their native state they are globular and upon denaturation, insoluble aggregates are formed.

\subsubsection{Cultured meat}

Tissue-engineering techniques can be used to in vitro culture animal muscle cells, which may then be transformed into meat (Langelaan et al., 2010; Post, 2012). To culture muscle fibres, first myoblast cells should be harvested from the skeletal muscle from an animal. Subsequently, these myoblasts are replicated by a standard cell culture methodology using serum-supplemented medium with all the necessary nutrients, including amino acids, lipids, vitamins and salts, for cells to grow. When a sufficient number of cells is obtained, they are placed onto a scaffold with anchor points to connect and align the cells, yielding a multicellular tissue. Electric fields or other means are often proposed to ensure alignment and 'normal' development of muscle fibres. After approximately three weeks, muscle fibres have matured and can be harvested. These fibres are $2-3 \mathrm{~cm}$ long and less than $1 \mathrm{~mm}$ thick. For upscaling this technique, a large-scale reactor is required in which the myoblasts can be cultured and exposed to the right environmental stimuli. The sensitivity of mammalian cells requires a high control over the growing conditions in the reactor and contamination should be avoided. Currently, these muscle fibres have been used to make a single hamburger as a proof of concept (Post, 2014).

\subsection{Comparison of techniques}

\subsubsection{Bottom-up \& top-down strategies}

The various techniques follow either a bottom-up or a top-down strategy to create the fibrous morphology (Fig. 8.1). With a bottom-up strategy, individual structural elements are created that are subsequently assembled into larger products. This strategy follows the structural organization of muscle with a hierarchy from macro- to nanoscale, being muscle cells, myofibrils, sarcomeres with the proteins myosin and actin, connected via conjunctive tissue (Pearson, 2012). These structural elements can be grown by cell culturing or biomass production of filamentous fungi; or by creating protein fibrils or fibres, which can be similar in size to the myofibrils, by wet or electrospinning. These fibrils/fibres can subsequently be assembled by cross-linking with 
enzymes, or mixing with binding agents, such as egg protein, and gluten (Davies \& Lightowler, 1998; Heuvel et al., 2013). To create similar structural anisotropy as in meat, the individual elements should be aligned prior to assembly. Scaffolds are used to let cells grow in one direction into a muscle meat with anisotropic properties. For the other bottom-up strategy, directional processing is used to align and assemble the individual structural elements.

Bottom-up strategy

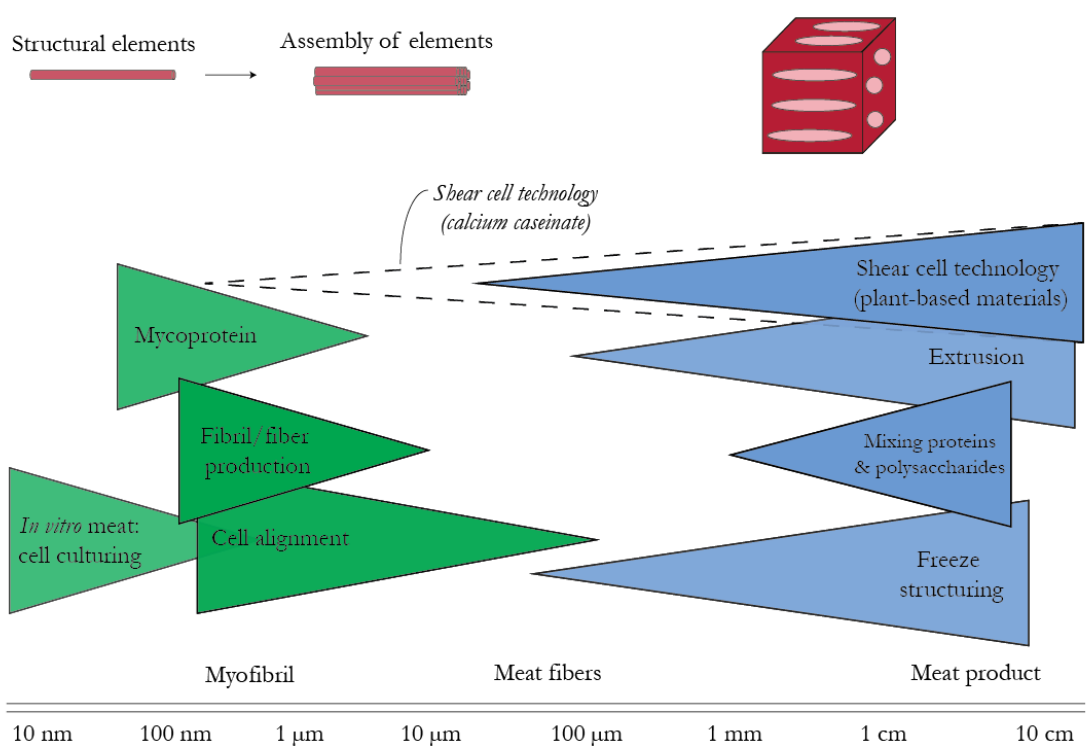

Top-down strategy

Shear cell technology

Fig. 8.1 Bottom-up (green) and top-down (blue) strategy to create fibrous products for the application of meat analogues.

Other techniques follow a top-down strategy, by mimicking the structure on larger length scales only. Therefore the structures obtained with these techniques mimic the structure, but do not fully resemble the meat structure in its hierarchical architecture. A force field is used to direct the self-assembly or aggregation of biopolymer(s), resulting in anisotropic structure on a micrometre scale. Unidirectional freezing directs the growth of ice crystals, which become aligned. Other techniques use a shear force to create anisotropy. These processes can be illustrated in a simplified way by assuming a two component system (Fig. 8.2), consisting of biopolymers in water. The two components do not mix, therefore one phase forms the continuous and the other a dispersed phase. Sheared or uni-directionally freezing such blends may deform the dispersed phase and align it in one direction. Besides deformation, the dispersed phase can also undergo coalesce and break-up, and even inversion of the two phases, which will affect the final properties of the material. After solidification, the fibrousness in the products 
originates from a continuous phase (mostly consisting of proteins) that is disrupted by a dispersed, deformed, weak phase: filaments that are matrix/structure breakers. This affects the mechanical properties of the material. Recently, a correlation was made between the deformation of the dispersed phase, i.e. the microstructural anisotropy, the mechanical anisotropy, and the fibrous macrostructure (Dekkers, Hamoen, et al., 2018). Furthermore, the volume fraction and size of the dispersed weak phase determine the properties of the final products (Dekkers et al., 2016).

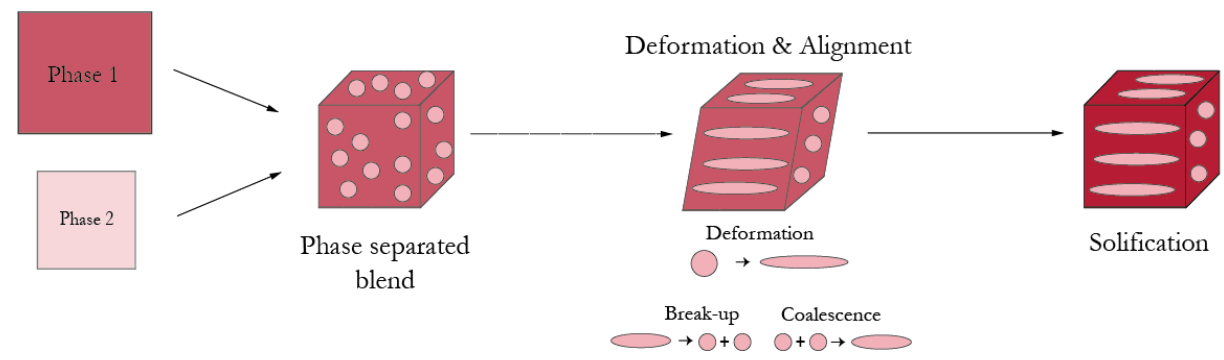

Fig. 8.2 Graphical illustration of a phase separated biopolymer blend, which forms a water-in-water (water-in-water) emulsion, which shows droplet coalescence, break-up and deformation.

\subsubsection{Strengths \& weaknesses}

Table 8.1 provides an overview of the different structuring techniques with the required ingredients and equipment, commercial availability, and compares them in terms of product properties, and process robustness, scalability, and resource efficiency. The main strength of the bottom-up strategy is that individual structural components are assembled such that they truly resemble the hierarchical structure of meat down to a nanoscale and thereby replicate the structure of meat in great detail. Cultured meat is a biomimetic material, which in terms of structural aspects comes potentially closest to animal meat (Post \& Hocquette, 2017). Structuring techniques with a top-down strategy mimic the structure on larger length scale only, and the question is whether the structure of the meat analogues, produced with a top-down strategy, sufficiently mimic the structure of real meat to entice the consumer into purchasing these products.

The downside of resembling the fibrous structure of meat from the nanoscale up, is that the environmental impact is typically higher (Smetana, Mathys, Knoch, \& Heinz, 2015), and the process robustness and scalability is more complex as compared to techniques that adopt a topdown strategy. There is a lot of optimism around cultured meat, although it is not yet clear how the scaling to commercial production should be done, and how the use of resources should be 
reduced such, that it can become competitive with other meat analogues. Researchers acknowledge that the production of cultured meat is resource-intensive because it requires highly refined ingredients and micronutrients in its media and well defined, sterile process conditions at all steps (Post \& Hocquette, 2017). Research on wet spinning for the application of meat analogues has been limited, presumably because of technical challenges such as the large waste water streams (Manski, van der Goot, \& Boom, 2007a). Freeze structuring is very energy intensive and this has impeded commercial-scale application until now, although there is some renewed attention to application on the level of catering, within the context of molecular gastronomy. The process is applied in other areas, such as for the creation of porous scaffolds for cell cultures, and for creating coatings or membranes (Cuadros, Erices, \& Aguilera, 2015; J. Wu \& Meredith, 2014). The other techniques following a top-down strategy have lower environmental impact.

The starting materials to be used are a major determinant of the efficiency in all methods. Cultured meat and the production of mycoprotein require a relatively small amount of stem cells, spores or fungi, but require large amounts of specific growth medium. In all other techniques, (plant) protein isolates and concentrates, potentially supplemented with some additional polysaccharides/hydrocolloids, are used as ingredients. Here, a distinction can be made in terms of the required purity and the functional properties of the proteins; for spinning, and freeze structuring, protein isolates are used (protein purity $>90 \%$ ), whereas in extrusion and simple shear flow, protein concentrates or protein isolate blends can be used. The use of less refined ingredients is better, since in the process less material is lost (Goot et al., 2016).

The combination of scalability and resource efficiency may render the techniques with a topdown strategy, and especially those that use a shear force to create anisotropy in biopolymer blends, the most viable option for replacing meat on the intermediate term, provided that the structure sufficiently mimics the structure of real meat. In the next sections, we will therefore further discuss these structuring techniques. These processes are in fact applied for many other products as well, and we will assess how these application may also yield better insight in protein structuring. This is connected with the capacity to quantify the structure, and therefore, methods for structure characterisation are outlined as well. 
Table 8.1 Overview of techniques to make meat analogues. The plus-sign (+) refers to an advantage, the minus-sign (-) refers to a disadvantage, the plus-minus-sign refers to a neutral aspect of the tecbnique, and a question (?) mark indicates that no reference was found.

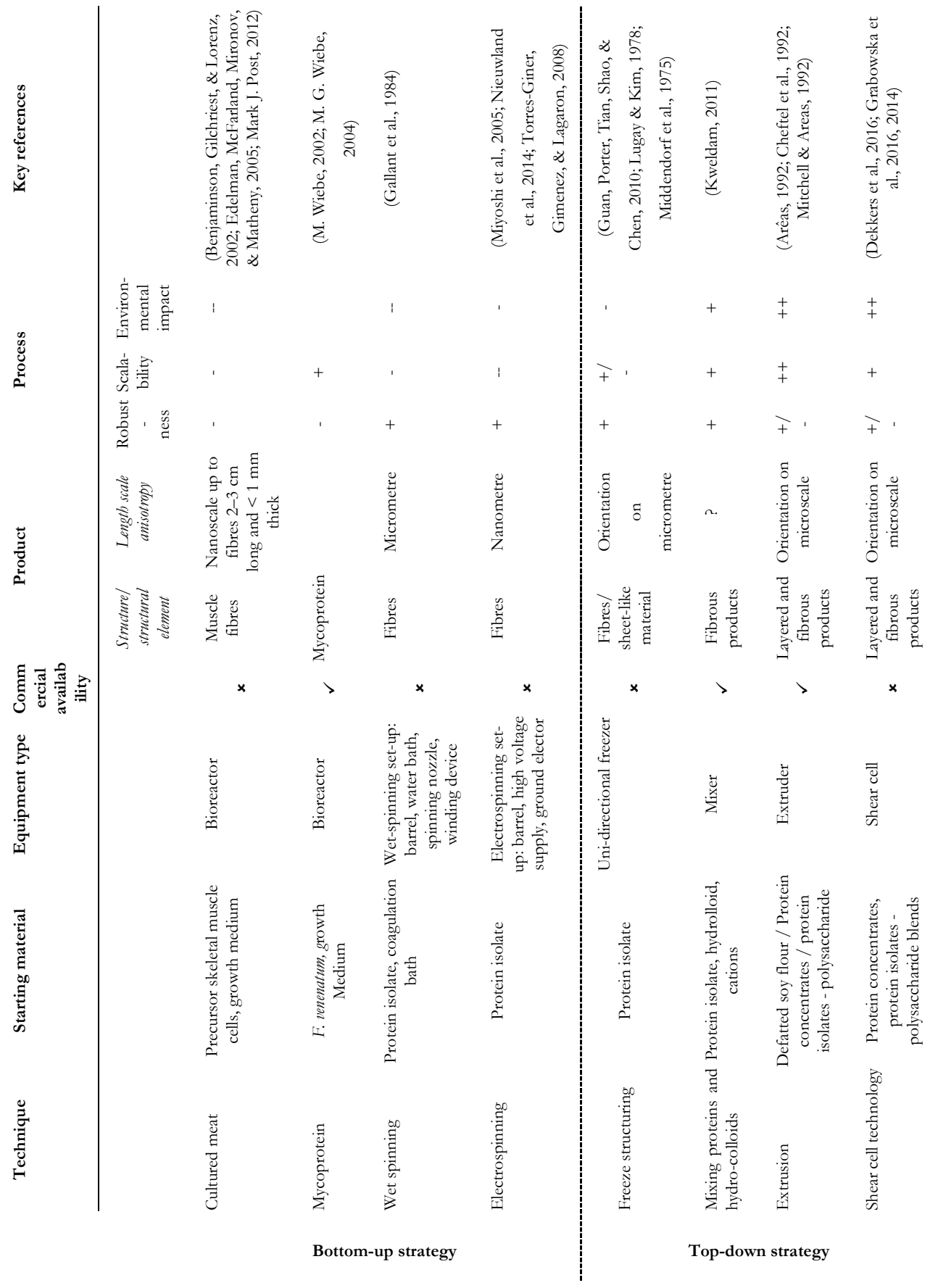




\subsection{Phenomena in directed assembly processes}

Structuring of phase separated, blended (bio)polymers by application of shear is studied extensively for other applications. The deformation of diluted biopolymer blends was studied for food and personal care applications (Erni et al., 2009; Sadeghi, Kadkhodaee, Emadzadeh, \& Phillips, 2018; Wolf et al., 2000). The coalescence of dispersed phase droplets was studied on larger scales in extrusion processes and couette cells for molten polymer blends, mostly for the applications of composite materials in construction (Elmendorp \& Maalcke, 1985; Puyvelde \& Moldenaers, 2005). These materials are not fibrous, but are composite, aligned materials, and the phenomena during these processes may shed light on the phenomena occurring during fibrous protein structuring. Therefore, we review the literature on shear-induced deformation, break-up, and coalescence of both diluted and concentrated (bio)polymer blends.

\subsubsection{Phase separated (bio)polymer blends}

(Bio)polymers generally do not (molecularly) mix at elevated concentrations, due to their very low entropy of mixing. Biopolymers are often water soluble or swell considerably in water, and form two aqueous phases when blended, forming a so-called water-in-water emulsion. After blending of the biopolymers, water will migrate between the two phases, leading to a redistribution of water and a change in both the volume fraction and consistency of the phase(s). This redistribution depends on the temperature, process history, and presence of other components (e.g. salts or sugars). It is therefore important to know the concentration of the biopolymer in each phase in such a water-in-water, after the biopolymers were blended (Capron, Costeux, \& Djabourov, 2001).

\subsubsection{Deformation and break-up}

Shear-induced deformation and break-up of water-in-water emulsions is regulated by the same phenomena as in oil-in-water or water-in-oil emulsions. The main difference between the two types of emulsions is the low interfacial tension (between $10^{-7}-10^{-5} \mathrm{~N} / \mathrm{m}$ ) of water-in-water emulsions as compared to emulsions with oil and water, resulting in droplets that are much more easily deformed than in emulsions with oil (Erni et al., 2009). Further, biopolymer phases exhibit more complex rheological behaviour. Factors that influence droplet deformation and break-up are the shear rate, the viscosities of both phases, and the size of the dispersed droplet. The 
viscosity ratio between the continuous and dispersed phase must be balanced to induce droplet deformation (Grace, 1982).

\subsubsection{Coalescence}

At low volume fractions of the dispersed phase, the interactions between droplets can often be neglected. In case of a higher dispersed phase volume fraction, coalsecence starts to play a role resulting in larger dispersed domains. This coalescence process can be described with four consecutive steps: $i)$ approach of two droplets, ii) drainage of the film between the two droplets, iii) break-up of this film, and iv) relaxation of the newly formed, merged droplet (Elmendorp \& Van der Vegt, 1986). The approach of two droplets depends on the collision probability of droplets, which, for orthokinetic coalescence, increases with the dispersed phase volume fraction, time and deformation rate. For two droplets to collide, the interaction time between two droplets should be longer than the time required for film drainage. The film ruptures when the film between two droplets is sufficiently thin (Chesters, 1991).

\subsubsection{Alignment}

Flow can align particles and fibres, thereby inducing macroscopic change in structure and properties. For example, many reinforced polymeric materials for construction are produced from a mixture of polymer and short fibres via extrusion, injection or compression moulding, yielding highly aligned composites (Ku, Wang, Pattarachaiyakoop, \& Trada, 2011). The fibres become aligned during processing, due to the shear flow, and strengthen the material lengthwise (Pickering, Efendy, \& Le, 2016). The mechanical performance of the final material is affected by the type of fibre, the adhesion between the fibres and the matrix, and the fibre distribution and orientation. Fibre alignment is dependent on the concentration and thus interaction between the fibres, the type of flow during processing, and the fibre aspect ratio, which is the ratio between the length and width of a fibre. Depending on the aspect ratio, fibres may exhibit a tumbling motion, or rotate toward the flow direction and become aligned. Similarly, (colloidal) particle alignment can be achieved through shearing of particle suspensions (Vermant, 2001). In case of (nearly) hard spheres, particles can only be aligned, whereas softer particles or droplets with liquid-like behaviour can be deformed by the shear flow and will aligned in the shear field with their long axis predominantly towards the direct of the shear (Belzung, Lequeux, Vermant, \& Mewis, 2000). 


\subsection{Methods to study directed assembly processes of multiphase systems}

Structuring via the deformation of a demixed biopolymeric solution can be largely understood from the previously discussed mechanisms of a phase separated blend in which the dispersed phase is deformed and aligned, and partial coalescence of the disperse phase(s). To predict whether a biopolymer blend can be structured, by deformation of the dispersed phase, several parameters can be determined. In comparison to diluted blends, different analytical methods are required. In this section, we will describe the state of the art in analytical methods to study directed assembly processes. Furthermore, the final (micro)structures obtained after solidification can be studied with various methods for further understanding of phenomena in structure formation processes.

\subsubsection{Methods for predicting deformation of biopolymer blends}

The deformability of the dispersed phase is dependent on the viscosity of the two phases, and the viscosity of the two phases is in turn dependent on the concentration of biopolymer in each phase, and as indicated in section 8.4.1, the concentration of the biopolymer in each phase, after the biopolymers have been blended, should be known.

\subsubsection{Water distribution in concentrated biopolymer blends}

The thermodynamic incompatibility of biopolymers in diluted blends has been studied extensively in the seventies and eighties (Antonov, Grinberg, \& Tolstoguzov, 1977; Antonov, Grinberg, Zhuravskaya, \& Tolstoguzov, 1980). Phase diagrams were derived by dissolving both biopolymers in water, and subsequently these biopolymers were separated by centrifugation, yielding a two-phase liquid with one phase rich in one biopolymer and poor in the other biopolymer, and vice versa. For more concentrated blends, centrifugation to separate the two phases is not practical, because the viscosities are too high, and we have use other methods to determine the phase composition. Rheological measurement can be used for this, via the polymer blending law that was developed by Takanyangi (Morris, 2009; Takayanagi, Harima, \& Iwata, 1963):

$$
M_{X Y}^{n}=\phi_{X} M_{X}^{n}+\phi_{Y} M_{Y}^{n}
$$

with $M$ being the modulus, $\phi$ the volume fraction of phase $\mathrm{X}$ and phase $\mathrm{Y}$, and $n$ an exponent that is dependent on how the phases react upon deformation; isostrain $(n=1)$, isostress $(n=-1)$ 
or bicontinuous $(\mathrm{n}=0.2)$. The modulus depends on the biopolymer concentration in each phase. A mass balance can be used to determine the theoretical values of the water concentrations in both phases (Clark, Richardson, Ross-Murphy, \& Stubbs, 1983). The modulus of the blend and of the consituent phases is used to determine the solvent partitioning, referred to as the solventavidity parameter or ' $\mathrm{p}$-factor', defined as

$$
p=\left(w_{x} / x\right) /\left(w_{y} / y\right)
$$

in which $w_{x}$ refers to the water in phase $\mathrm{X}, w_{y}$ refers to the water in phase $\mathrm{Y}, x$ is the amount of polymer $\mathrm{X}$, and $y$ is the amount of polymer Y. This approach has been used extensively to determine the partitioning of different solvents (Katopo, Kasapis, \& Hemar, 2012; Shrinivas, Kasapis, \& Tongdang, 2009), but in 1983, Clark et al. already recognized that the uncertainties in determining the paritioning are quite large (Clark \& House, 1987).

More direct methods to determine water distribution were proposed. The first method is by determining the Flory-Huggins polymer-solvent interaction parameter $\chi$, and calculating the distribution between the two phases. This method is most accurate when applied at water activities that are not too high; but at high water activities this method becomes relatively inaccurate and dependent on the crosslink density and other factors. A second method relies on determining the phase volume with microscopy and extensive image analysis. This relies on images that are not necessarily representative of the total material. A third method is by directly measuring the properties of the solutions, by time domain nuclear magnetic resonance (TDNMR). TD-NMR was used to determine solvent partitioning in concentrated soy protein isolate - wheat gluten dispersions (Dekkers et al., 2016). Water distributes unevenly in this blend; soy protein absorbs more water than gluten, leading to a much lower protein concentration in the latter phase. In a follow-up study, the water distribution after a heat and a heat-shear treatment was determined (Dekkers, Emin, Boom, \& van der Goot, 2018). Water distribution was not effected by these treatments after cooling, but it is still unclear how water distributes between biopolymers during heating, which would be relevant for understanding shear based structuring processes. Measuring this is still beyond the current possibilities.

\subsubsection{Viscoelastic properties}

The prediction of the deformability of concentrated water-in-water emulsions is complex, because of the conditions used during structuring (concentration, temperature, pressure). Therefore classical rheometers cannot be used. Instead, different rheometric systems have been 
proposed to determine the viscoelastic properties of (bio)polymers, while mimicking the conditions during structuring: the capillary die rheometer, the on-line slit die rheometer, and the closed cavity rheometer. The capillary die viscometer was used to determine the melt viscosity (Jasberg et al., 1982; Remsen and Clark, 1978), but for non-Newtonian fluids, entrance effects complicate the interpretation of the measurements. Slit die viscometers are mounted at the end of the extruder and measure the pressure gradient to thereby determine the shear viscosity under the assumptions of Newtonian flow and no wall slip (Horvat, Azad Emin, Hochstein, Willenbacher, \& Schuchmann, 2013; Li, Campanella, \& Hardacre, 2004). The closed cavity rheometer (CCR) is a rheometer that can be used under similar conditions as applied during structuring in terms of temperature and pressure, however this rheometer applies oscillatory instead of steady shear (Emin and Schuchmann, 2017). Furthermore, the shear cell, can, besides structuring, also be used as a rheometer, since torque values are recorded during rotation of the cone (Emin et al., 2012); however the accuracy of these values is low.

\subsubsection{Methods for analysing structure}

Optical, mechanical, dielectric, and imaging methods (Damez \& Clerjon, 2008) are available for the study of meat structure. Some of these methods can be applied to determine the structure and anisotropy in meat analogues as well. Mechanical methods are employed to measure the anisotropy both in meat and in meat analogues, however these methods are invasive. Anisotropy is also investigated with other methods such as light reflectance, fluorescence polarization spectroscopy, X-ray tomography (XRT) and spin-echo small angle neutron scattering (SESANS), which leaves the material intact.

\subsubsection{Macrostructure}

While macroscopic imaging is perhaps the best method to obtain an intuitive feeling of the structure, it only allows subjective assessment. Pictures of fibrous products obtained with extrusion, mixing, and the shear cell technology are shown in Fig. 8.3. Although all three methods use shear to deform and align biopolymers, the (macro)structure of the products is different. In the extruded samples, a V-shaped pattern is observed. Individual layers are oriented parallel to the die wall. The structure of the materials prepared by mixing proteins, hydrocolloids and metal cations, is more random, and less coherent as compared to the other structures. The macrostructure of samples prepared with the shear cell technology show distinct fibres in the direction of the shear flow. 

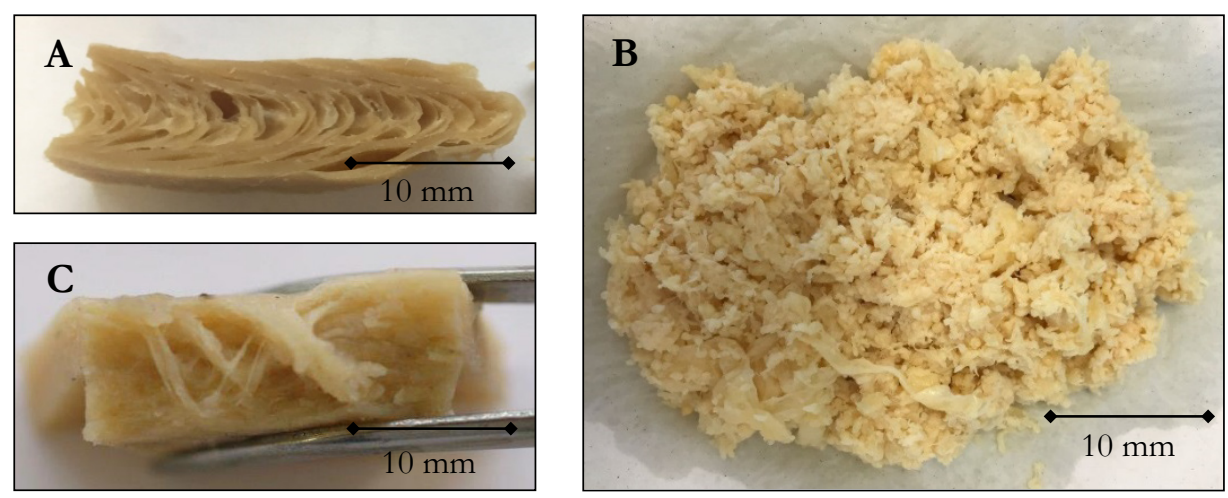

Fig. 8.3 Macrostructure of A) extruded sample from soy protein concentrate B) a sample prepared by mixing lupine proteins, alginate and calcium and C) a soy protein isolate - wheat gluten sample from the shear cell

\subsubsection{Mechanical methods}

Mechanical methods allow quantification of the mechanical anisotropy. The Warner-Bratzler Shear Force (WBSF) is the most widely used method to determine the tenderness of meat. In this test, the force needed to move a knife to tear or cut a piece of meat is measured to determine the hardness (tenderness) of meat. The analysis of this hardness is, for other foods, often done with texture profile analysis (TPA). The material is here compressed, which provides not only the hardness but also the springiness, cohesiveness, gumminess, and chewiness. TPA has been used sparingly in the analysis of meat (Ruiz De Huidobro, Miguel, Blázquez, \& Onega, 2005), but several studies performed TPA for meat analogues (Chen, Wei, Zhang, \& Ojokoh, 2010; Lin, Huff, \& Hsieh, 2000, 2002; Rehrah, Ahmedna, Goktepe, \& Yu, 2009). Besides TPA, the mechanical anisotropy may also be determined by performing large deformation tests, such as tensile tests. The anisotropy of materials is quantified by measuring sample properties in two directions; parallel and perpendicular to the fibres. In samples obtained with extrusion, the fibres are due to $\mathrm{V}$-shaped structures with the tip being in the direction of the flow through the die. The fibrous products created with simple shear flow deformation have their anisotropy in the direction of the shear flow. These samples can be teared apart to determine the tensile strength of the materials (Chen, Wei, Zhang, \& Ojokoh, 2010; Grabowska et al., 2014; Seiichiro \& Noguchi, 1987).

\subsubsection{Protein-protein interactions}

The fibrous products obtained with extrusion processing of protein blends, are believed to originate from specific cross-linking between proteins. Therefore protein-protein interactions in structured products have been studied extensively (Burgess \& Stanley, 1976; Liu \& Hsieh, 2008; 
Osen, Toelstede, Eisner, \& Schweiggert-Weisz, 2015). These interactions are generally classified into covalent, hydrophilic-hydropobic interactions, and disulfide bonds, which can be investigating by applying reagents that break non-covalent protein-protein interactions. Although this method is used widely, there no conclusive understanding on which types of interactions, hydrophobic/hydrophilic interactions or disulfide bonding, are the most important in the formation of structure.

\subsubsection{Microscopy}

The deformation of the dispersed phase in fibrous products has been studied with several types of microscopy: light microscopy, scanning electron microscopy (SEM) and confocal scanning laser microscopy (CSLM). Some examples are given in Fig. 8.4. In Fig. 8.4 A-D, proteins are stained with either coomassie brilliant blue or rhodamine B. In all micrographs, the proteins, being the black structures in Fig. 8.4 A-C and red in Fig. 8.4 D, are found to be aligned in the shear flow direction. The microstructure depicted in Fig. 8.4 C shows extruded soy flour with oil - the black spots are oil droplets, which are dispersed throughout the protein matrix. Similarly, polysaccharide (cellulose) stained with calcofluor white (blue spots) is dispersed throughout the protein matrix (red fibres) when soy protein concentrate was structured with the shear cell technology (Fig. 8.4 D). The structures depicted in Fig. 8.4 F-H are imaged with SEM, in which the samples are dried prior to imaging. The drying step alters the microstructure, and therefore some samples were fixated prior to drying. Similarly to Fig. 8.4 B and D, protein forms the continuous and polysaccharide (pectin) the dispersed phase. In all these micrographs, alignment is observed along the shear direction, resulting in microstructural anisotropy. 

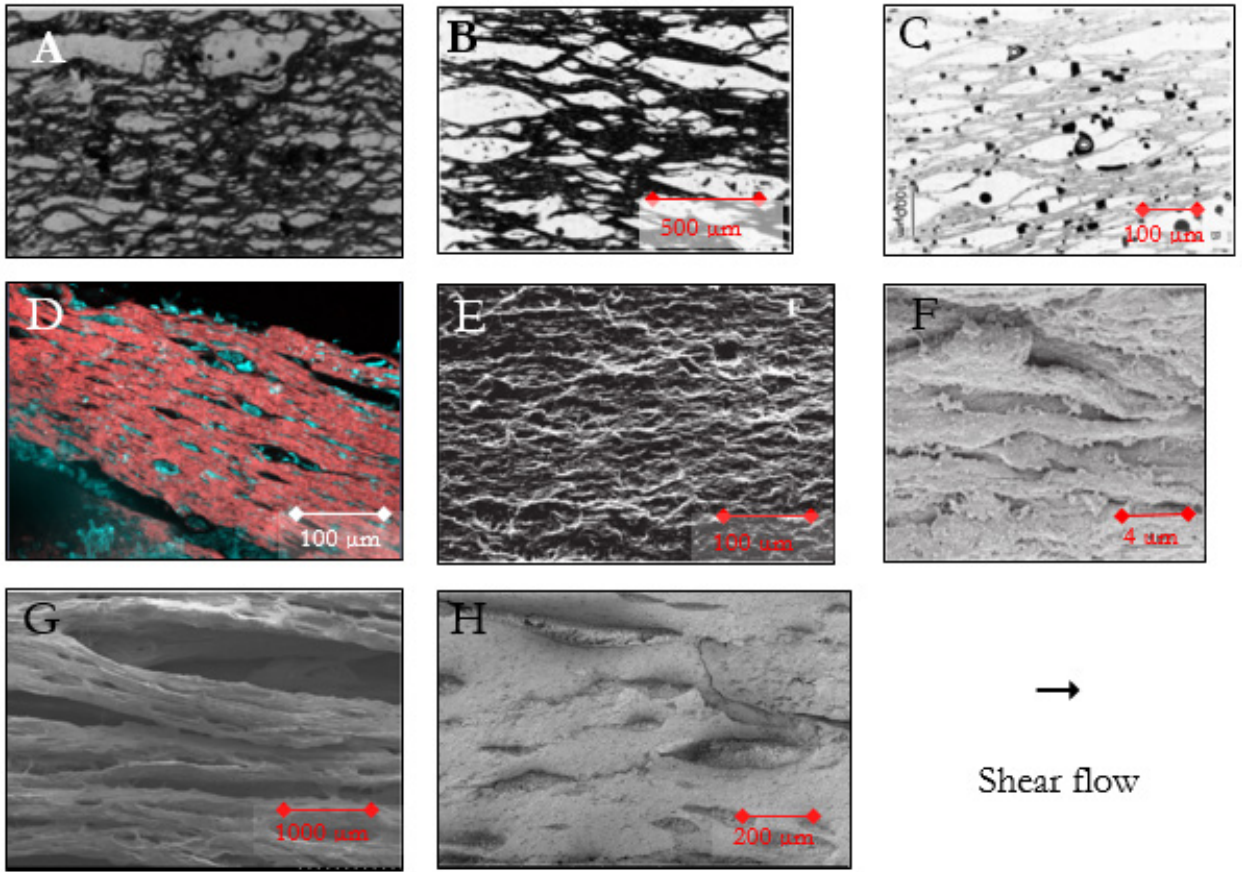

Fig. 8.4 Micrographs from fibrous samples A) Extruded soy flour (Seïchiro \& Noguchi, 1987) B-C) Extruded defatted soy flour with added oil (Gwiazda, Noguchi, \& Saio, 1987), D) Soy protein concentrate structured with simple shear flow deformation (Grabowska et al., 2016) E) Extruded Soy protein isolate (SPI) - starch blend (Lin et al., 2002), F) SPI wheat gluten (WG) blend structured in a conical shear cell (Grabowska et al., 2014), G) SPI-WG blend structured in a conette cell (Krintiras et al., 2015), H) SPI - pectin blend structured in a conical shear cell (Dekkers, Hamoen, Boom, do van der Goot, 2018).

\subsubsection{Light reflectance}

The random-walk theory of light reflectance and refraction in anisotropic media may be used to determine anisotropy (Weiss, Porra, \& Masoliver, 1998). In anisotropic samples, the probability of a photon to assume a certain direction differs along the different axes. The reflectance intensity of samples shows an ellipse in isotropic samples, whereas for anisotropic samples the reflectance intensity deviates from this elliptical shape. Samples with various degrees of fiber formation after extrusion were tested and a good correlation was found between the fibrousness of a sample and the deviation from this ellipse (Ranasinghesagara, Hsieh, Huff, \& Yao, 2009; Ranasinghesagara, Hsieh, \& Yao, 2006).

\subsubsection{Fluorescence polarization}

Fluorescence polarization spectroscopy utilises polarized light to excite fluorescent molecules. Tryptophan is a natural fluorescent probe, and used in meat science to determine the meat structure. Similarly, this technique has been used to assess the anisotropy of extruded materials 
(Yao, Liu, and Hsieh, 2004). An optimal moisture content at 60 wt.\% was found to yield an optimum in degree of polarization degree and anisotropy, which correlates well with results from other methods.

\subsubsection{X-ray tomography}

$\mathrm{X}$-ray tomography or XRT can detect differences in material density. In meat science, XRT is mostly used to distinguish bone, lean meat and fat, but it can also be used to determine air that is entrapped during structuring in a meat analogue. In extrusion processes, expansion of the material due to water evaporation is partly responsible for the final product properties. Also in structuring with the shear cell technology, air is entrapped in the product. In a study on structuring SPI-pectin blends, an air volume fraction of approximately $10 \mathrm{v} . \%$ was found. These air bubbles form elongated capillaries in the shear flow direction (Dekkers, Hamoen, et al., 2018), and these air pockets may contribute to the fibrous properties of the samples.

\subsubsection{Spin-echo small-angle neutron scattering}

Spin-echo small-angle neutron scattering (SESANS) is a technique that was introduced as a complementary technique to determine the anisotropy in fibrous materials (Krintiras et al., 2014). It is based on neutron diffraction and can distinguish structures over three orders of magnitude - from $10 \mathrm{~nm}$ up to $10 \mu \mathrm{m}$. Neutron diffraction depends on the number and shape of the density fluctuations that the neutron beam encounters. In analysing structures with SESANS, it was assumed that the fibres are bundles of cylinders, and that these fibres are composed of material (protein) other than the phase that surrounds them. The refraction along interfaces was different along the direction of the fibres as compared to perpendicular to the fibre direction, resulting in differences in depolarization.

\subsubsection{Impediments for structure analysis}

The preceding section may have illustrated that significant progress has been made on the methods to analyse structured products, but in situ analysis the formation process is still a challenge. In diluted dispersions, deformation of the dispersed phase can be analyzed with rheooptics: for example Lundell et al., used small angle light scattering (SALS) used to determine the extension of droplets while under deformation (Lundell, de Hoog, Tromp, \& Hermansson, 2005). However, for more concentrated blends, which are turbid, in situ investigations are not trivial. 
In addition, most analytical methods are oriented towards specific technical aspects, and have not been directly linked to sensory attributes. In meat science, meat structure is often correlated to sensory attributes. For example, the tenderness of meat is related to the distribution of connective tissue throughout the meat, and juiciness of meat products is related to the water holding capacity (Damez \& Clerjon, 2008). Similar correlations between biophyical methods and sensory attributes are required to understand what technical characteristics are important for the sensory experience of consumers. Sensory and general consumer research should answer the question to which extent the structure of meat analogues, produced with a top-down strategy, should mimic the structure of real meat to entice the consumer into purchasing these products.

\subsection{Conclusions}

There are different processing routes to create fibrous products, which can be classified in bottom-up and top-down strategies. A bottom-up strategy starts with the formation of individual structural components from individual molecules including proteins, which are then assembled into larger structures and products. With a top-down strategy, mostly blends of proteins and/or polysaccharides are mixed to create anisotropic, fibrous products through directed assembly. The structure that is created becomes apparent when deforming or stretching the material. From a structural point of view, the bottom-up strategy has the potential to mimic meat most closely, whereas from a process design point of view, top-down strategy is more robust, scalable and have better resource efficiency. While the last decades have seen tremendous development in the characterisation of structured foods, the further development of the top-down processing strategies will require new in situ analytical methods to follow the structuring process itself. The combined evolution of both analytical methods and structuring processes will enable us to create meat analogues that have the desired sensory properties, combined with excellent resource efficiency.

\section{Acknowledgements}

We thank all members of the Plant Meat Matters consortium for the valuable discussions. This research is part of the project Plant Meat Matters, which is co-financed by Top Consortium for Knowledge and Innovation Agri \& Food by the Dutch Ministry of Economic Affairs; the project is registered under contract number TKI-AF-16011. 


\section{Chapter 9}

General discussion 


\subsection{Introduction}

One strategy to reduce meat consumption is the development of meat analogues that resemble the original meat product. The anisotropic, fibrous nature of meat is perhaps its most important characteristic. In this thesis, fibrous structure formation with the shear cell technology was studied for plant-based ingredients to create in-depth understanding of the process dynamics and the resulting product properties. This thesis reports on the use of different approaches to create those novel insights. In Chapter 2, 3 and 6, analytical methods were developed and employed to study concentrated biopolymer blends under conditions that are relevant for fibrous structure formation. Additional insight was created by systematically varying the process conditions during structure formation process in the shear cell (Chapter 4 and 5). Further, the importance of the properties of the raw material were highlighted by adapting the process to obtain a protein concentrate from soy meal. Aqueous fractionation was used as a sustainable method to produced soy protein concentrates with different properties, which gave more insight on the desired properties of the starting material (Chapter 7). Lastly, literature was reviewed to put all findings in perspective and to understand the key success factors to make the next generation meat analogues (Chapter 8). This chapter (Chapter 9) summarizes the main findings of the preceding chapters. Subsequently, design rules for fibrous structure formation with the shear cell technology are given. Besides, challenges, compromises and efforts to study the structuring processes, in terms of ingredients and process, are described. The chapter ends with an outlook on the developments in this field.

\subsection{Main findings and conclusions}

Phase-separated biopolymer blends, such as a blend of soy protein isolate (SPI) and wheat gluten (WG), can be transformed into a fibrous product with the shear cell. However, the product structure that is obtained depends on the dry matter content and the ratio between SPI and WG (Grabowska et al., 2014). Fibrous structure formation requires two phases at least to have a sufficiently high viscosity to allow transfer of stresses onto the material, resulting in deformation, alignment and entrapment of the two phases during shearing. The viscosity of the two phases depends on the amount of water in each phase. Thus, we developed an approach based on time domain nuclear magnetic resonance relaxometry (TD-NMR) to assess the absorbed water in the separate SPI and WG phases when blended in concentrated dispersions (Chapter 2). It was shown that water redistributes in SPI - WG blends; more water was absorbed by the SPI as compared to the WG phase. Although this water distribution was shown to be hardly affected 
by a heat or shear treatment (Chapter 3), the viscoelastic properties of the two phases changed significantly. The viscoelastic properties of SPI and WG became more similar due to water redistribution in the blend, which explained deformation and alignment of the dispersed phase during structuring.

In Chapter 4, 5 and 6, a SPI - pectin blend was investigated as a model blend for soy protein concentrate (SPC), which is known to give a good fibrous structure when sheared and heated. Pectin is a soluble polysaccharide, and in combination with SPI, fibrous materials were obtained at a similar heating temperature as SPC, being $140{ }^{\circ} \mathrm{C}$ (Chapter 4). Pectin formed the dispersed phase and was deformed when heated and sheared at optimal conditions. Furthermore, the addition of pectin to SPI resulted in products in which approximately 10 v. $\%$ air was incorporated (Chapter 5). The fibrous nature of these products appears upon tearing, and originates from detachment through or along the long side of the weak dispersed phase(s), being pectin and/or air. The size and orientation of the dispersed phases, tailored by using different shear rates, was related to differences in fracture behaviour when deforming the structures. Besides deformation, the strength and volume fraction of the weak phase(s) is important when composing a blend for fibrous structure formation. Although one can clearly discern two distinct phases with SEM, the viscoelastic behaviour of the SPI-pectin blend is more complex than that of a simple composite material (Chapter 6). The addition of a small amount of pectin (2.2 wt.\%) to a SPI dispersion (41.8 wt.\%) resulted in viscoelastic behaviour that changes in time, when determined during a shear treatment at elevated temperatures.

In Chapter 7, aqueous-fractionated material from soy was used for fibrous structure formation. Fibrous structures were obtained when: $i$ ) the soy protein fractions were toasted, which is a dry heating step, and ii) when a concentrate ( $75 \mathrm{wt} . \%$ protein) was combined with full fat flour (37 wt. $\%$ protein), in such a ratio that the protein content was similar to commercial SPC (63 wt.\%). The functional properties of these soy protein materials were determined and some of the essential functional properties for fibrous structure formation were revealed. Toasting resulted in a decreased nitrogen solubility, increased water holding capacity and increased the viscoelastic properties of the fractions, which seems to be important for fibrous structure formation.

Chapter 8 summarizes different techniques to create meat-like structures for the application of meat analogues. The techniques are discussed by classifying the techniques as either bottom-up or top-down. The advantages and disadvantages of the approaches and the techniques have been 
outlined by analysing the structures and their (potential) resemblance to meat, robustness, scalability, and resource efficiency. The techniques that make use of directed assembly processes in biopolymer blends were further investigated by reviewing literature on similar processes when creating anisotropic products that are outside this particular field of application, i.e. not meant to create fibrous structures. These insights were subsequently translated to the conditions as used in structure formation for meat analogues.

The research described in these chapters contributes to a better understanding of fibrous structure formation process with the shear cell. Structure formation depends on the properties of the two phases in the blend. For successful structure formation, the properties of these two phases should be such that deformation of the dispersed phase(s) can take place during processing, and structure entrapments occurs due to the solidification upon cooling. In addition, the two phases have different strengths in the final product, the continuous phase being stronger than the dispersed phase, or the adhesion between the phases is poor.

\subsection{Design rules for fibrous structure formation with the shear cell technology}

At present, SPI - WG blends, SPI - pectin blends, and commercial and aqueous fractionated SPC have been successfully used to transform into fibrous materials. The study was focused on finding the generic behaviour of these blends, and these insights can therefore be used to derive rules for the starting material/ingredients, and the processing conditions in the shear cell (Fig. 9.1).

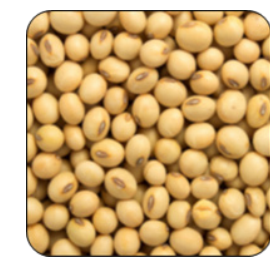

1. Ingredients

$\checkmark$ Plant-based

$\checkmark$ Concentrated blend consisting of protein(s) and/or polysaccharide(s)

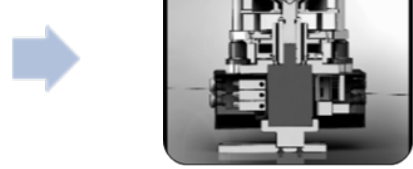

2. Process

$\checkmark$ Heating to achieve viscosity that allows transfer of stress to both phases and sufficient solidification upon cooling Shear rate to create deformation of a weak dispersed phase, limited break-up

Fig. 9.1 Design rules for fibrous structure formation with a shear cell

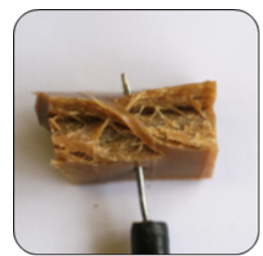

3. Product

Entrapped deformed microstructure

Weak dispersed phase or weak adhesion between phases 


\subsubsection{Ingredients: concentrated two-phase biopolymer blend}

A concentrated two-phase biopolymer blend is generally required for fibrous structure formation. The two phases are composed of mostly protein(s) and/or polysaccharides in water. These components can be naturally present in one ingredient (SPC) or assembled from two plant-based ingredients. Up till now, mostly fat-free ingredients have been used, or fat was a minor component (Chapter 7).

The ingredients that give a fibrous structure after processing, are mixed with water, such that there is incomplete hydration, yielding a crumbly dough. Until now, plant-based ingredients were successfully transformed into fibrous products, only when the amount of water that is added is lower than the water holding capacity (WHC) of the ingredients when measured in diluted dispersions. The way that the water interacts with the proteins and is entrapped in the network is therefore important to further investigate. This conclusion is mostly based on aqueous fractionated soy proteins. Those proteins were initially well dispersible in water (prior to a toasting pre-treatment), yielding paste-like products. Toasting was therefore used to alter the structuring properties of the ingredients (Chapter 7).

Besides the amount of water, also the order in which the various ingredients are hydrated in a blend was found important for structure formation. In the blends that contain SPI, the isolate was hydrated before adding the second component, being either WG or pectin, because preliminary experiments showed that this results in better structure formation. Especially for the SPI - pectin blends, a different dough was obtained when changing the order of hydration. Presumably, the soy proteins should have enough time to absorb the water in this way and therefore gain the right properties as compared to blending all dry ingredients and subsequently adding water. The exact reason for this phenomenon is not yet fully clear.

\subsubsection{Process: controlling deformation during processing}

During processing in the shear cell, shear is exerted on both the continuous and the dispersed phase, which gave deformation and alignment of the dispersed phase. Therefore the viscosity of the dispersed phase should not differ much from the viscosity of the continuous phase. One should bear in mind that in concentrated biopolymer blends, water redistributes unevenly, depending on the interactions of each biopolymer with water, and therefore the assumption that 
the original mass fraction is also the concentration of each biopolymer in a phase will lead to incorrect assumptions, as was shown for SPI-WG blends (Chapter 2 and 3).

The extent of deformation of the two phases depends on the interplay between the properties of the two phases and the process conditions, shear rate, temperature and time. For fibrous structure formation, the shear forces should be sufficiently high to deform the dispersed phase with no or only limited break-up of the dispersed phase (Chapter 5). The temperature during shearing has a direct effect on the viscosity and therefore on the deformability of both phases. Furthermore, heating induces (chemical) changes in the material, which impacts the viscosity and deformability over time indirectly as well, as shown in Chapter 7. Insight in the timetemperature dependency of those materials changes at the relevant concentrations and conditions will be required to understand the structuring potential for better control and the use of other plant protein materials.

\subsubsection{Product: understanding its properties}

We describe a fibrous product as a protein matrix, which is disrupted by a dispersed, deformed, weak phase: filaments that act as matrix breakers. When this dispersed phase is not or insufficiently deformed, an isotropic product is obtained, whereas sufficient deformation of the dispersed phase results in anisotropic properties. This dispersed, deformed phase may be weak, or the adhesion between the continuous and the dispersed phase may be weak. Upon stretching the material, it will break upon the deformed, dispersed phase, resulting in a fibrous appearance (Fig. 9.2). The dispersed phase is aligned in the shear flow direction, while there is no orientation in the other directions (vorticity and velocity).

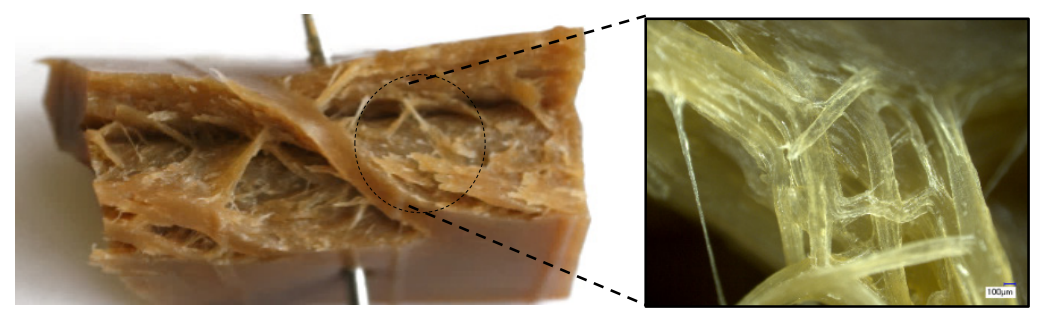

Fig. 9.2 Fibrous appearance of a product prepared from a soy protein isolate (SPI) - pectin blend at $140{ }^{\circ} \mathrm{C}$ in a conical shear cell.

In Chapter 5, we investigated the relation between mechanical properties and the deformation of the dispersed phase(s), pectin and/or air, in the product. A model is used to relate the dispersed phase properties (volume, size and deformation) to the resulting mechanical 
properties. When comparing model and experimental data, it was concluded that air must be mainly responsible for the anisotropy of the product, when one assumes that water distributes evenly between the SPI and the pectin phases. However, there was still a large discrepancy between the experimental and the predicted tensile stress. The differences between experimental and predicted tensile stress differences became smaller, when a larger volume fraction of the pectin phase was assumed, due to a redistribution of water over the SPI and pectin phase. Further investigations on the incorporation of air in the structures, and the deformation of air bubbles that are entrapped are required to be more conclusive on the relative importance of the presence of air and its role as a weak phase.

\subsection{Studying complex materials and conditions}

There are several reasons why creating insights in the shear cell technology is not straightforward. In most analytical methods, material or ingredients are characterized in solution, at room temperature, while in equilibrium. If needed, concentrations are adapted such that measurements can be performed well. In contrast, the starting materials/ingredients for fibrous structure formation are more complex: condensed dispersions, consisting of multiple components, which are (partly) insoluble, and which physical properties change during treatment at high temperatures. Unfortunately, the properties of these denser systems cannot simply be predicted by analysing the properties at lower concentrations or when analysing only the soluble fractions: their behaviour at higher concentrations, in more complex mixtures and at high temperatures is not just quantitatively, but also qualitatively different. One way to deal with at least part of this complexity is by simplifying the raw materials by using model systems. This is the subject of the next section. Furthermore, the process conditions during structuring add to the complexity, since structuring processes take place at elevated temperatures while shearing. Under these conditions, the properties of the biopolymers change over time. Hence, it is essential to study these properties and processes under similar conditions.

\subsubsection{Model systems for studying fibrous structure formation}

SPC can be considered as a mixture of components, of which protein, insoluble and soluble polysaccharides are most important. That is why SPI - pectin blends were used as a model system for SPC in this thesis. With this model system, insight was obtained in the deformation of the dispersed pectin and air phase, which would not (yet) have been possible for SPC. However, the 
comparability between this model system (SPI - pectin), and the natural protein concentrates (SPC) has not yet been discussed extensively.

The optimum shearing conditions were the same for both materials: a temperature of $140{ }^{\circ} \mathrm{C}$ (Chapter 4), a shear rate of $39 \mathrm{~s}^{-1}$ (Chapter 5), and similar shearing times (Grabowska et al., 2016). Furthermore, the amount of soluble polysaccharides added to SPI was taken similarly to the amount of soluble polysaccharides present in commercial SPC (roughly $3 \mathrm{wt} . \%$ in a product). However, the amount of proteins present in the final product is different, being $34.7 \mathrm{wt} . \%$ in the SPI-pectin blend (taking into account the protein content in SPI) and approximately 27.7 wt. $\%$ in the commercial SPC. The difference (roughly $7 \mathrm{wt} . \%$ ) is mostly caused by the presence of insoluble polysaccharides, such as cellulose, in SPC. Preliminary research showed that when adding cellulose (Sigma Aldrich, $20 \mu \mathrm{m}$ version (S3504)) as a third component to the SPI - pectin model blend, a structure was obtained with high anisotropy and a fibrous appearance (Fig. 9.3A). This anisotropy, as measured with a tensile strength test, was even higher than that obtained with SPI - pectin blends or commercial SPC (Grabowska et al., 2016). When adding the cellulose separately to SPI, macroscopic phase separation was obtained (Fig. 9.3B) with on the inside a similar structure to a SPI sample (Fig. 9.3C) and on the outside a white cellulose band. This behaviour may be similar to the earlier reported separation of wheat dough into gluten and (ungelatinised) starch in a shear cell (Zalm et al., 2009). Obviously, the presence of pectin prevents this migration of cellulose through the SPI matrix, and moreover in the presence of pectin, the cellulose enhances the anisotropy. Cellulose as present in SPC can be described as rigid rods, and we hypothesize that these rods align in the shear flow and therefore increase the anisotropy. These insights can subsequently be used in functionality driven fractionation processes, which would not aim to obtain pure fractions that are later to be combined again, but would aim at only adjusting the content of protein, soluble and insoluble polysaccharides such, that the desired functionality is obtained. This would allow the use of milder and more sustainable extraction conditions, which would contribute to better resource efficiency in the production of meat analogues (Geerts, 2018). 

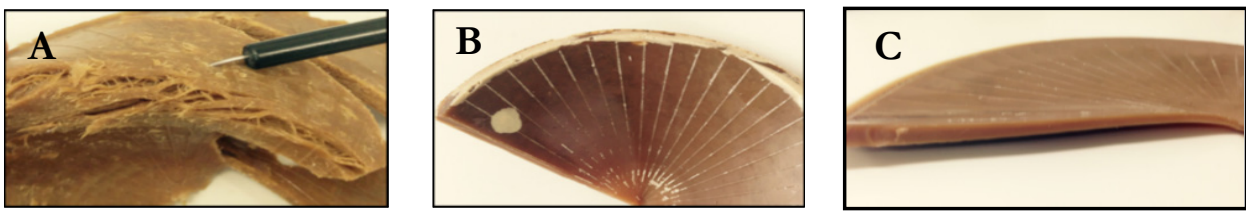

Fig. 9.3 Shear cell products consisting of A) soy protein isolate (SPI) - pectin-cellulose blend (38 wt.\% SPI $+6.5 \mathrm{wt} . \%$ pectin $+6.5 \mathrm{wt} . \%$ cellulose) B) SPI-cellulose blend (41 wt.\% SPI + $3 \mathrm{wt. \%}$ cellulose) C) SPI (44 wt.\%)

Nevertheless, the questions remains whether the SPI - pectin blend is representative (a good model system) for SPC. Chapter 7 describes experiments showing that the viscoelastic properties of the SPI-pectin blend are more complex than those of a simple composite material, which are thought to be due to chemical changes of both phases. For comparison, we performed similar measurements with SPC (Fig. 9.4). These experiments show that the viscoelastic properties of SPC are different from those of the SPI-pectin blend. While SPI-pectin shows an increase in $G^{\prime}$ in time, after an initial decrease, SPC only shows decreasing $G^{\prime}$-values in time. Furthermore, the $G^{\prime}$ and $G$ " of SPC are larger than those of the SPI-pectin blend at all temperatures.

We thus conclude that, while the model system has many similar properties, the differences with the original system are also large. One reason might be that the more intense processing needed to obtain SPI influences the properties of the protein in that material. In other words, already the protein phase alone might be different in SPI and SPC. Nevertheless, the model system clearly suggested insights for mechanisms of structure formation, rendering it a helpful model system, despite the fact that it did not provide a definite proof.
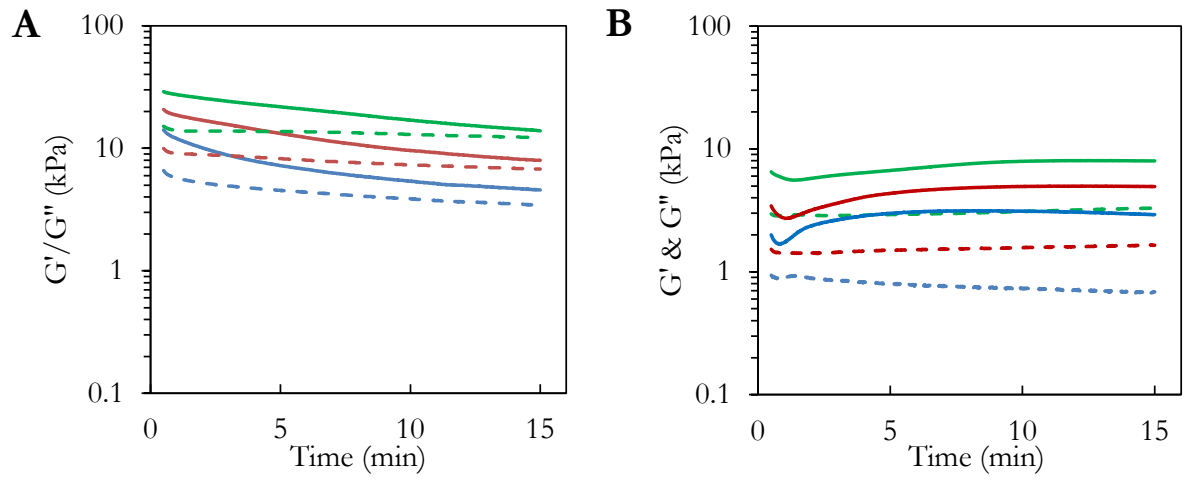

Fig. 9.4 Time-sweep measurement (79.65\% strain, $10 \mathrm{~Hz}$ frequency) performed in a closed cavity rheometer (CCR) at various temperatures (green $=120^{\circ} \mathrm{C}$, red $=130^{\circ} \mathrm{C}$, and blue $=140^{\circ} \mathrm{C}$ ) of A) soy protein concentrate and B) soy protein isolate (SPI) - pectin blend (Chapter 6, Fig. 1C), G' is solid line and G' is dashed line. 


\subsubsection{Measurements under relevant conditions - method development}

Measuring properties at relevant but unconventional conditions requires effort and compromise. We exemplify this by the measurements done with the closed cavity rheometer in Chapter 3, 6 and 7. In this rheometer, the material is heated and simultaneously subjected to a shear treatment by applying a high frequency and high strain in an oscillation experiment to approximate simple shear flow as applied in the shear cell. Though the use of this rheometer already represents a breakthrough in the possibilities to study the materials at relevant conditions, the behaviour of materials under oscillatory shear differs significantly from that during continuous rotational shear. However, the viscoelastic behaviour at these elevated temperatures is believed to be relevant for predicting the structuring behaviour, despite the fact that the exact interpretation of the measurements is far from trivial.

In Chapter 3, the water distribution in SPI - WG blends was determined before and after a heat and shear treatment. Here, we assumed that the water distribution while processing remains the same, and this water distribution was used for determining the viscoelastic behaviour with the isolated phases, at elevated temperatures. However, one can debate whether this assumption is valid. Hence, measuring the water distribution in protein blends at elevated temperatures and during processing would be very relevant. We envisage an experimental set-up, which allows measuring the properties of protein blends at elevated temperatures in a NMR spectrometer. Ideally, this NMR spectrometer is even combined with a rheometer to allow simultaneous measurements for further understanding of these temperature-time dependent effects.

The time-domain NMR and rheology approach (Chapter 2 and 3) as such cannot be used for materials that are natural mixtures, such as concentrates that consist of proteins and polysaccharides. For the natural mixtures, additional methods should be developed to estimate the water distributions and mechanical properties of the separate phases as present in these natural mixtures. The prediction of the behaviour of these natural blends is in fact even more complicated since these concentrates are actually multi-phase systems, instead of just being twophase systems.

Finally, further characterization of the products is required. Chapter $\mathbf{8}$ describes several analytical methods for determining the anisotropy with mechanical, optical, and imaging techniques for meat analogues in analogy to the characterization of meat. The quality and sensory attributes of meat are monitored and can be predicted with these biophysical methods. For 
example, meat toughness is related to the myofibrillar structure and connective tissue of meat, and juiciness is related to its water holding capacity (Damez \& Clerjon, 2008; Honikel, 1998). For plant-based meat analogue products though, the relation between these biophysical methods and the resulting sensory attributes is not established yet.

\subsection{Future prospects}

The overall societal goal of developing meat analogue food products is to help consumers in the transition from animal-based to a more plant-based diet. Therefore, the scientific goal to obtain insight in fibrous structure formation as developed in this thesis and other scientific research/publications is of importance, and can be the basis for the next generation meat analogues. However, before the resulting technology can be commercialized, there are still some scientific issues to resolve.

\subsubsection{Controlling deformation during processing}

During the shear cell process, the heat-shear treatment is followed by a cooling step. Up to now, no shear was applied during cooling. This is different in case of extrusion processing, where the material is probably structured when flowing through the cooling die. The investigation of the effects of shear during cooling in a shear cell could be employed for testing two competing hypotheses: i) isotropic products are the result of a blend that cannot be deformed during shearing, or ii) isotropic products are the result of a two-phase system that is deformed during the shearing process, but the material recovers faster than the entrapment of the structure proceeds during cooling. When the last hypothesis is valid, then one could optimize the entrapment of a deformed structure, and therefore create a larger window for successful structure formation. For example, faster cooling may increase the viscosities faster and thus entrap the deformation before recovery, or the shear treatment may be continued during cooling. Another idea could be to ensure entrapment of the continuous phase by adding extra components that solidify immediately upon cooling below a specific temperature.

\subsubsection{Sensory attributes of meat analogues}

This thesis contributed to a better understanding of structure formation processes with the shear cell technology. The fibrous products created with this technology can form the basis for the next generation meat analogue products. However, more is needed. Although the product structure is very important in mimicking the original meat product, also other sensory attributes, 
such as colour, flavour and juiciness, are important for developing successful meat analogue products (Chapter 8 ).

Ideally, colorants, flavours or the precursors of flavours are added with the other ingredients in the shear cell to avoid complexity in the production process. However, colorants and flavours can be sensitive to the high temperatures applied during processing and can evaporate, degrade or react with the matrix (proteins) in an undesired way. Therefore stable precursors might be required that develop the final flavour during the heat (and shear) treatment. When the aim would be to mimic the colour of meat as closely as possible, a colour change during cooking is desired. The product should be red-pinkish prior to preparation and become red-brownish upon heating. However, it is not trivial to realize this, especially when aiming for an easy process in which the colorants are added simultaneously with the other components, since the heat treatment in the shear cell will already result in the colour transition.

Juiciness is often related to the water holding capacity and the retention of water in meat. The role of water is different during the several steps of preparation and consumption. In case of meat there is drip loss during preparation, water loss upon cooking/heating, and water release upon mastication. The moisture loss during cooking of meat is the result of the denaturation of proteins upon heating resulting in a change in water holding of the meat. The structures made with the shear cell technology have a network that is already formed; the product has been heated before final preparation prior to consumption. As a result the type of network will change less compared to that of meat during cooking. Juiciness during mastication is related to the way in which water is bound in the protein network and its release when a force is applied by chewing the product. For example, water, which is entrapped in capillaries, will be released faster than water entrapped in a protein network (Reig, Lillford, \& Toldrá, 2008). The moisture that is released upon mastication is not only important for the juiciness, but also for flavour release. Flavours that are dissolved in the water, or that are dissolved in a fat phase being emulsified in water, will be released with the water, and hence the water phase should be released gradually to ensure continued sensation of the flavours during mastication similarly to meat. A fast(er) release of the water phase will result in an experience of the product as being dry and rubbery before swallowing. 


\subsubsection{Other plant materials}

Up to now, soy and wheat gluten are the main ingredients for commercial meat analogue production (Asgar et al., 2010). Both ingredients have their disadvantages, and ideally, also other plant-based ingredients are used for fibrous structure formation: starch-rich pulses such as pea, lentil, or faba bean, oil-rich seeds such as sunflower or rapeseed, or other crops such as potato or quinoa. The use of other plant-based ingredients could be used to create a larger variety in products and cater to different nutritional or sensorial needs. These plant proteins have different functional properties and therefore the translation of the process from soy and wheat gluten towards these raw materials is not straightforward. The design rules (described in this chapter) are meant as a starting point to make this translation a more systematic process, than was possible until now.

\subsubsection{Nutritional value meat analogue products}

In the transition from animal-based to plant-based proteins, the difference in nutritional value should be considered. Meat is a source of heme-iron and vitamin B12. Heme-iron is more bioavailable as compared to nonheme-iron as present in plants. Furthermore, individuals that hardly consume animal-derived foods are at risk for vitamin B12 deficiency. Fully vegetarian/vegan diets might require the use of supplements for these micronutrients. However, when consumers adopt a flexitarian or a semi-vegetarian diet, supplementation is generally speaking less of an issue since the dietary reference intake is already met when consuming a relatively low quantity of animal products (Food and Nutrition Board, Institute of Medicine, 2011).

In addition to being a source of micro-nutrients, meat has a high protein content and a balanced essential amino acid composition (Biesalski, 2005). Meat analogues based on plant-based proteins can be designed such that they have an equivalent protein content. However, proteins from a single plant source have a sub-optimal amino acid balance, meaning that the plants are limited in certain essential amino acids, such as lysine in cereal grains and sulphur-containing amino acids in oil seeds or legumes (Day, 2013). Pairing several plant protein sources is a strategy to ensure sufficient consumption of essential amino acids. Furthermore, when replacing animalbased proteins with plant-based proteins, the digestibility of the proteins should be considered. The digestibility of plant proteins is often evaluated with a protein digestibility-corrected amino acid score (PDCAAS), which is calculated by ratio of the amount of limiting amino acid in $1 \mathrm{~g}$ 
of test protein divided by the reference protein. The PDCAAS of soy protein was ranked only slightly lower (PDCAAS = 91) than animal-derived proteins from for example milk and eggs, and a larger difference in the PDCAAS was determined for other plant proteins, such as proteins from legumes or wheat (Schaafsma, 2000).

Finally, processing impacts the nutritional value of meat analogue products. Ingredient extraction (Chapter 8), and processing (heating and shearing) may affect the digestibility of the proteins due to cross-linking and oxidation reactions. Furthermore, in protein concentrates, both proteins and (reducing) sugars are present that can react via the Maillard reaction and subsequently form advanced glycation end products (AGEs). AGEs can affect inflammatory markers, and are associated with late complications in diabetes (Poulsen et al., 2013). However, to what extent these reactions impact the nutritional value of meat analogues is unknown.

It should be noted that a more plant-based diet does not necessary perform worse than current diets, rich in animal products. For example, dietary fibre intake is low in current diets, and can increase when shifting towards a more plant-based diet. Besides, micronutrients like polyphenols can also enrich the current diet. Overall, the diets will become more balanced when increasing intake from plant-based products. Of course, the net effect depends on the type of consumer and his/her diet. It is clear though that more research is required to investigate the effect high inclusion level of (processed) plant-based products, including meat analogues on the nutritional profile of those diets.

\subsection{Concluding remarks}

The overall aim of this thesis was to obtain in-depth understanding of the key mechanisms in the creation of an anisotropic, fibrous structure in plant-based biopolymer blends by applying shear deformation. Therefore, the properties of these biopolymer blends were analysed prior to, during, and after the structure formation process. It was concluded that for successful structure formation, matching of the properties of the two phases is key. During structuring, the two phases are deformed, upon cooling, the (continuous) phase solidifies ensuring entrapment of the deformed dispersed phase(s). For fibrous appearances of the product, it seems necessary that the continuous and dispersed phase have different strength and in the final product at eating temperature. Future research should confirm these findings also for other (plant-based) ingredients. 
While the complexity in these systems makes full and quantitative understanding a continuing challenge, it is also clear that this same complexity is the origin of much of the richness in the behaviour of the blends. Further study into the role of this complexity, and into finding the balance between retaining the emergent properties, and still being able to measure and understand the behaviour, is practically very important, and scientifically very exciting. 


\section{References}


Ahmed, S., \& Jones, F. R. (1990). A review of particulate reinforcement theories for polymer composites. Journal of Materials Science, 25, 4933-4942. http://doi.org/10.1007/BF00580110

Akdogan, H. (1999). High moisture food extrusion. International Journal of Food Science and Technology, 34, 195 207.

Ako, K., Durand, D., \& Nicolai, T. (2011). Phase separation driven by aggregation can be reversed by elasticity in gelling mixtures of polysaccharides and proteins. Soft Matter, 7(6), 2507. http://doi.org/10.1039/c0sm01152e

Albersheim, P., \& Neukom, H. (1960). Splitting of pectin chain molecules in neutral solutions. Archives of Biochemistry and Biophysics, 90, 46-51. http://doi.org/10.1016/0003-9861(60)90609-3

Antonov, Y. A., Grinberg, V. ., \& Tolstoguzov, V. B. (1977). Phase equilibria in water-proteinpolysaccharide systems II. Water-casein-neutral polysaccharide systems. Colloid \& Polymer Science, $255,2-5$.

Antonov, Y. A., Grinberg, V. Y., Zhuravskaya, N. a, \& Tolstoguzov, V. B. (1980). Liquid two-phase waterprotein-polysaccharide systems and their processing into textured protein products. Journal of Texture Studies, 11, 199-215.

Apaiah, R. K., Linnemann, A. R., \& Van Der Kooi, H. J. (2006). Exergy analysis: A tool to study the sustainability of food supply chains. Food Research International, 39(1), 1-11. http://doi.org/10.1016/j.foodres.2005.04.006

Arêas, J. A. (1992). Extrusion of food proteins. Critical Reviens in Food Science and Nutrition, 32(4), 365-392. http://doi.org/10.1080/10408399209527604

Asgar, M. A., Fazilah, A., Huda, N., Bhat, R., \& Karim, A. A. (2010). Nonmeat Protein Alternatives as Meat Extenders and Meat Analogs. Comprehensive Reviews in Food Science and Food Safety, 9(5), 513-529. http://doi.org/10.1111/j.1541-4337.2010.00124.x

Axelos, M. A. V., \& Branger, M. (1993). The effect of the degree of esterification on the thermal stability and chain conformation of pectins. Food Hydrocolloids, 7(2), 91-102. http://doi.org/10.1016/S0268005X(09)80161-6

Belzung, B., Lequeux, F., Vermant, J., \& Mewis, J. (2000). Flow-induced anisotropy in mixtures of associative polymers and latex particles. Journal of Colloid and Interface Science, 224(1), 179-187. Retrieved from http://www.scopus.com/inward/record.url?eid=2-s2.00034176808\&partnerID $=40 \& \mathrm{md} 5=83 \mathrm{e} 3 \mathrm{a} 4851 \mathrm{c} 045305 \mathrm{ee} 9 \mathrm{ec} 44260 \mathrm{~b} 62 \mathrm{fab}$

Bengoechea, C., Arrachid, A., Guerrero, A., Hill, S. E., \& Mitchell, J. R. (2007). Relationship between the glass transition temperature and the melt flow behavior for gluten, casein and soya. Journal of Cereal Science, 45(3), 275-284. http://doi.org/10.1016/j.jcs.2006.08.011

Berk, Z. (1992). Technology of production of edible flours and protein products from soybeans.

Biesalski, H. K. (2005). Meat as a component of a healthy diet - Are there any risks or benefits if meat is avoided in the diet? Meat Science, 70(3 SPEC. ISS.), 509-524. http://doi.org/10.1016/j.meatsci.2004.07.017

Boland, M. J., Rae, A. N., Vereijken, J. M., Meuwissen, M. P. M., Fischer, A. R. H., van Boekel, M. A. J. S., ... Hendriks, W. H. (2013). The future supply of animal-derived protein for human consumption. Trends in Food Science and Technology, 29(1), 62-73. http://doi.org/10.1016/j.tifs.2012.07.002 
Bosmans, G. M., Lagrain, B., Deleu, L. J., Fierens, E., Hills, B. P., \& Delcour, J. a. (2012). Assignments of proton populations in dough and bread using NMR relaxometry of starch, gluten, and flour model systems. Journal of Agricultural and Food Chemistry, 60(21), 5461-5470. http://doi.org/10.1021/jf3008508

Burgess, L. ., \& Stanley, D. . (1976). A Possible Mechanism for Thermal Texturization of Soybean Protein. Canadian Institute of Food Science and Technology Journal, 9(4), 228-231. http://doi.org/10.1016/S0315$5463(76) 73681-2$

Campbell, K. A., Glatz, C. E., Johnson, L. A., Jung, S., De Moura, J. M. N., Kapchie, V., \& Murphy, P. (2011). Advances in aqueous extraction processing of soybeans. JAOCS, Journal of the American Oil Chemists'Society, 88(4), 449-465. http://doi.org/10.1007/s11746-010-1724-5

Campbell, M. F. (1981). Processing and product characteristics for textured soy flours, concentrates and isolates. Journal of the American Oil Chemists' Society, 58(3), 336-338. http://doi.org/10.1007/BF02582372

Capron, I., Costeux, S., \& Djabourov, M. (2001). Water in water emulsions: Phase separation and rheology of biopolymer solutions. Rheologica Acta, 40(5), 441-456. http://doi.org/10.1007/s003970100161

Cheftel, J. C., Kitagawa, M., \& Queguiner, C. (1992). New protein texturization processes by extrusion cooking at high moisture levels. Food Reviews International, 8(2), 235-275.

Chen, F. L., Wei, Y. M., Zhang, B., \& Ojokoh, A. O. (2010). System parameters and product properties response of soybean protein extruded at wide moisture range. Journal of Food Engineering, 96(2), 208213. http://doi.org/http://dx.doi.org/10.1016/j.jfoodeng.2009.07.014

Chen, J., Liang, R. H., Liu, W., Liu, C. M., Li, T., Tu, Z. C., \& Wan, J. (2012). Degradation of high-methoxyl pectin by dynamic high pressure microfluidization and its mechanism. Food Hydrocolloids, 28(1), $121-$ 129. http://doi.org/10.1016/j.foodhyd.2011.12.018

Chen, J., Liu, W., Liu, C.-M., Li, T., Liang, R.-H., \& Luo, S.-J. (2015). Pectin modifications: a review. Critical Reviews in Food Science and Nutrition, 55(12), 1684-1698. http://doi.org/10.1080/10408398.2012.718722

Chesters, A. K. (1991). The modelling of coalescence process in fluid-liquid dispersions: A Review of Current Understanding. Chemical Engineering Research and Design, 69(4), 259-270.

Clark, A. H., \& House, C. (1987). Structural and Mechanical Properties of Biopolymer Gels. Biopolymers: Advances in Polymer Science, 57-192. http://doi.org/10.1007/BFb0023332

Clark, A. H., Richardson, R. K., Ross-Murphy, S. B., \& Stubbs, J. M. (1983). Structural and mechanical properties of agar/gelatin co-gels. Small-deformation studies. Macromolecules, 16(8), 1367-1374. http://doi.org/10.1021/ma00242a019

Cuadros, T. R., Erices, A. A., \& Aguilera, J. M. (2015). Porous matrix of calcium alginate/gelatin with enhanced properties as scaffold for cell culture. Journal of the Mechanical Behavior of Biomedical Materials, 46, 331-342. http://doi.org/10.1016/j.jmbbm.2014.08.026

Damez, J.-L., \& Clerjon, S. (2008). Meat quality assessment using biophysical methods related to meat structure. Meat Science, 80(1), 132-49. http://doi.org/10.1016/j.meatsci.2008.05.039

Damez, J.-L., \& Clerjon, S. (2013). Quantifying and predicting meat and meat products quality attributes using electromagnetic waves: an overview. Meat Science, 95(4), 879-96. 
http://doi.org/10.1016/j.meatsci.2013.04.037

Davies, J., \& Lightowler, H. (1998). Plant-based alternatives to meat. Nutrition \& Food Science, 90-94.

Day, L. (2013). Proteins from land plants - Potential resources for human nutrition and food security. Trends in Food Science \& Technology, 32(1), 25-42. http://doi.org/10.1016/j.tifs.2013.05.005

De Almeida, N. M., De Moura Bell, J. M. L. N., \& Johnson, L. A. (2014). Properties of soy protein produced by countercurrent, two-stage, enzyme-assisted aqueous extraction. JAOCS, Journal of the American Oil Chemists'Society, 91(6), 1077-1085. http://doi.org/10.1007/s11746-014-2436-z

De Boer, J., Schösler, H., \& Aiking, H. (2014). "Meatless days" or "less but better"? Exploring strategies to adapt Western meat consumption to health and sustainability challenges. Appetite, 76, 120-128. http://doi.org/10.1016/j.appet.2014.02.002

de Moura, J. M. L. N., Campbell, K., De Almeida, N. M., Glatz, C. E., \& Johnson, L. A. (2011). Protein recovery in aqueous extraction processing of soybeans using isoelectric precipitation and nanofiltration. JAOCS, Journal of the American Oil Chemists' Society, 88(9), 1447-1454. http://doi.org/10.1007/s11746-011-1803-2

De Roeck, A., Duvetter, T., Fraeye, I., Plancken, I. Van der, Sila, D. N., Loey, A. Van, \& Hendrickx, M. (2009). Effect of high-pressure/high-temperature processing on chemical pectin conversions in relation to fruit and vegetable texture. Food Chemistry, 115(1), 207-213. http://doi.org/10.1016/j.foodchem.2008.12.016

Dekkers, B. L., Emin, M. A., Boom, R. M., \& van der Goot, A. J. (2018). The phase properties of soy protein and wheat gluten in a blend for fibrous structure formation. Food Hydrocolloids, 79, 273-281. http://doi.org/10.1016/j.foodhyd.2017.12.033

Dekkers, B. L., Hamoen, R., Boom, R. M., \& van der Goot, A. J. (2018). Understanding fiber formation in a concentrated soy protein isolate - pectin blend. Journal of Food Engineering, 222, 84-92.

Dekkers, B. L., Kort, D. W. de, Grabowska, K. J., Tian, B., As, H. Van, \& Goot, A. J. van de. (2016). A combined Rheology and Time Domain NMR approach for determining water distribution in Protein Blends. Food Hydrocolloids, 60, 525-532. http://doi.org/10.1017/CBO9781107415324.004

Dekkers, B. L., Nikiforidis, C. V., \& Goot, A. J. van der. (2016). Shear-induced fibrous structure formation from a pectin / SPI blend. Innovative Food Science and Emerging Technologies, 36, 193-200. http://doi.org/10.1016/j.ifset.2016.07.003

Diaz, J. V., Anthon, G. E., \& Barrett, D. M. (2007). Nonenzymatic degradation of citrus pectin and pectate during prolonged heating: Effects of $\mathrm{pH}$, temperature, and degree of methyl esterification. Journal of Agricultural and Food Chemistry, 55(13), 5131-5136. http://doi.org/10.1021/jf0701483

Dubey, R. K., \& Bhattacharya, S. (2015). Extrusion processing of foods. In Conventional and Advanced Food Processsing Technologies (pp. 75-97).

Dunn, P. J., Wells, A., \& Williams, M. T. (2010). Green Chemistry in the pharamaceutical industry. John Wiley \& Sons.

Elmendorp, J. J., \& Maalcke, R. J. (1985). A study on polymer blending microrheology: Part 1. Polymer Engineering and Science Science, 25(16), 1041-1047.

Elmendorp, J. J., \& Van der Vegt, A. K. (1986). A study on polymer blending microrheology: part IV. The influence of coalescence on blend morphology origination. Polymer Engineering and Science, 26(19), 
1332-1338. http://doi.org/10.1002/pen.760261908

Elzerman, J. E., Boekel, M. A. J. S. van, \& Luning, P. A. (2013). Exploring meat substitutes: consumer experiences and contextual factors. British Food Journal, 115(5), 700-710. http://doi.org/doi:10.1108/00070701311331490

Elzerman, J. E., Hoek, A. C., van Boekel, M. a. J. S., \& Luning, P. a. (2011). Consumer acceptance and appropriateness of meat substitutes in a meal context. Food Quality and Preference, 22(3), 233-240. http://doi.org/10.1016/j.foodqual.2010.10.006

Elzerman, J. E., Hoek, A. C., van Boekel, M. J. A. S., \& Luning, P. A. (2015). Appropriateness, acceptance and sensory preferences based on visual information: A web-based survey on meat substitutes in a meal context. Food Quality and Preference, 42, 56-65. http://doi.org/10.1016/j.foodqual.2015.01.010

Emin, M. A., Quevedo, M., Wilhelm, M., \& Karbstein, H. P. (2017). Analysis of the reaction behavior of highly concentrated plant proteins in extrusion-like conditions. Innovative Food Science \& Emerging Technologies, (September), 0-1. http://doi.org/10.1016/j.ifset.2017.09.013

Emin, M. A., Schmidt, U., van der Goot, A. J., \& Schuchmann, H. P. (2012). Coalescence of oil droplets in plasticized starch matrix in simple shear flow. Journal of Food Engineering, 113(3), 453-460. http://doi.org/10.1016/j.jfoodeng.2012.06.015

Emin, M. A., \& Schuchmann, H. P. (2017). A mechanistic approach to analyze extrusion processing of biopolymers by numerical, rheological, and optical methods. Trends in Food Science \& Technology, 60, 88-95. http://doi.org/10.1016/j.tifs.2016.10.003

Erni, P., Cramer, C., Marti, I., Windhab, E. J., \& Fischer, P. (2009). Continuous flow structuring of anisotropic biopolymer particles. Advances in Colloid and Interface Science, 150(1), 16-26. http://doi.org/10.1016/j.cis.2009.05.005

Facca, A. G., Kortschot, M. T., \& Yan, N. (2006). Predicting the elastic modulus of natural fibre reinforced thermoplastics. Composites Part A: Applied Science and Manufacturing, 37(10), 1660-1671. http://doi.org/10.1016/j.compositesa.2005.10.006

Firoozmand, H., Murray, B. S., \& Dickinson, E. (2009). Interfacial structuring in a phase-separating mixed biopolymer solution containing colloidal particles. Langmuir, 25(19), 1300-1305. http://doi.org/10.1021/la8037389

Firoozmand, H., \& Rousseau, D. (2014). Tailoring the morphology and rheology of phase-separated biopolymer gels using microbial cells as structure modifiers. Food Hydrocolloids, 42, 1-11. http://doi.org/10.1016/j.foodhyd.2014.04.040

Fitzsimons, S. M., Mulvihill, D. M., \& Morris, E. R. (2008). Co-gels of whey protein isolate with crosslinked waxy maize starch: Analysis of solvent partition and phase structure by polymer blending laws. Food Hydrocolloids, 22, 468-484. http://doi.org/10.1016/j.foodhyd.2007.01.011

Fraeye, I., De Roeck, A., Duvetter, T., Verlent, I., Hendrickx, M., \& Van Loey, A. (2007). Influence of pectin properties and processing conditions on thermal pectin degradation. Food Chemistry, 105(2), 555-563. http://doi.org/10.1016/j.foodchem.2007.04.009

Galbraith, H. (2002). Hormones in international meat production: biological, sociological and consumer issues. Nutrition Research Reviews, 15(2), 293. http://doi.org/10.1079/NRR200246

Geerts, M. (2018). Functionality-driven fractionation: The need for mild food processing. 
Giezen, F. E., Jansen, W. W. J. T., \& Willemsen, J. H. A. (2014). Method of making structured protein compositions.

Goot, A. J. van der, Pelgrom, P. J. M., Berghout, J. a. M., Geerts, M. E. J., Jankowiak, L., Hardt, N. A., ... Boom, R. M. (2016). Concepts for further sustainable production of foods. Journal of Food Engineering, 168, 42-51. http://doi.org/10.1016/j.jfoodeng.2015.07.010

Grabowska, K. J., Tekidou, S., Boom, R. M., \& van der Goot, A.-J. (2014). Shear structuring as a new method to make anisotropic structures from soy-gluten blends. Food Research International, 64(0), 743-751. http://doi.org/http://dx.doi.org/10.1016/j.foodres.2014.08.010

Grabowska, K. J., Zhu, S., Dekkers, B. L., De Ruijter, N. C. A., Gieteling, J., \& Van Der Goot, A. J. (2016). Shear-induced structuring as a tool to make anisotropic materials using soy protein concentrate. Journal of Food Engineering, 188, 77-86. http://doi.org/10.1016/j.jfoodeng.2016.05.010

Grace, H. P. (1982). Dispersion Phenomena in High Viscosity Immiscible Fluid Systems and Application of Static Mixers As Dispersion Devices in Such Systems. Chemical Engineering Communications, 14(36), 225-277. http://doi.org/10.1080/00986448208911047

Grinberg, V. Y., \& Tolstoguzov, V. B. (1997). Thermodynamic incompatibility of proteins and polysaccharides in solutions. Food Hydrocolloids, 11(2), 145-158. http://doi.org/10.1016/S0268005X(97)80022-7

Grunert, K. G., Bredahl, L., \& Brunsø, K. (2004). Consumer perception of meat quality and implications for product development in the meat sector - A review. Meat Science, 66(2), 259-272. http://doi.org/10.1016/S0309-1740(03)00130-X

Guido, S., Simeone, M., \& Alfani, A. (2002). Interfacial tension of aqueous mixtures of Na-caseinate and Na-alginate by drop deformation in shear flow. Carbobydrate Polymers, 48(2), 143-152. http://doi.org/10.1016/S0144-8617(01)00220-X

Gwiazda, S., Noguchi, A., \& Saio, K. (1987). Microstructural Studies of Texturized Vegetable Protein Products : Effects of Oil Addition and Transformation of Raw Materials in Various Sections of a Twin Screw Extruder, 6(1).

Habeych, E., Dekkers, B., van der Goot, A. J., \& Boom, R. (2008). Starch-zein blends formed by shear flow. Chemical Engineering Science, 63(21), 5229-5238. http://doi.org/10.1016/j.ces.2008.07.008

Habeych, E., van der Goot, A. J., \& Boom, R. (2009). In situ compatibilization of starch-zein blends under shear flow. Chemical Engineering Science, 64(15), 3516-3524. http://doi.org/10.1016/j.ces.2009.04.034

Harper, J. M., \& Clark, J. P. (1978). Food extrusion. CRC Critical Reviens in Food Science and Nutrition, 11(2), 155-215. http://doi.org/10.1080/10408397909527262

Hartmann, C., \& Siegrist, M. (2017). Consumer perception and behaviour regarding sustainable protein consumption: A systematic review. Trends in Food Science \& Technology, 61, 11-25. http://doi.org/10.1016/j.tifs.2016.12.006

Hermansson, A.-M. (1985). Structure of Soya Glycinin and Conglycinin Gels. Journal of the Science of Food and Agriculture, 36(9), 822-832.

Hermansson, A. M. (1986). - Soy Protein Gelation. Journal of the American Oil Chemists' Society, 63(5), 658666.

Heuvel, M. Van Den, Geerdink, P., Brier, P., Eijnden, P. Van Den, Henket, J. T. M. M., Marloes, L. P., ... 
Martin, A. H. (2013). Food-grade electrospinning of proteins, (April), 9-12.

Hoek, A. C., Luning, P. A., Weijzen, P., Engels, W., Kok, F. J., \& de Graaf, C. (2011). Replacement of meat by meat substitutes. A survey on person- and product-related factors in consumer acceptance. Appetite, 56(3), 662-673. http://doi.org/10.1016/j.appet.2011.02.001

Honikel, K. O. (1998). Reference methods for the assessment of physical characteristics of meat. Meat Science, 49(4), 447-457. http://doi.org/10.1016/S0309-1740(98)00034-5

Horvat, M., Azad Emin, M., Hochstein, B., Willenbacher, N., \& Schuchmann, H. P. (2013). A multiplestep slit die rheometer for rheological characterization of extruded starch melts. Journal of Food Engineering, 116(2), 398-403. http://doi.org/10.1016/j.jfoodeng.2012.11.028

Huang, H. C., Chang, T. C., \& Jane, J. (1999). Mechanical and physical properties of protein-starch based plastics produced by extrusion and injection molding. Journal of the American Oil Chemists' Society, 76(9), 1101-1108. http://doi.org/10.1007/s11746-999-0210-4

Islas-Rubio, A. R., \& Higuera-Ciapara, I. (2002). Soy beans : Post-harvest Operations.

Kakade, M. L., Rackis, J. J., McGhee, J. E., \& Puski, G. (1974). Determination of trypsin inhibitor activity of soy product: a collaborative analysis of an improved procedure. American Association of Cereal Chemists, 51, 376-382.

Karr-Lilienthal, L. K., Kadzere, C. T., Grieshop, C. M., \& Fahey, G. C. (2005). Chemical and nutritional properties of soybean carbohydrates as related to nonruminants: A review. Livestock Production Science, 97(1), 1-12. http://doi.org/10.1016/j.livprodsci.2005.01.015

Kasapis, S., \& Al-Marhoobi, I. M. (2005). Bridging the divide between the high- and low-solid analyses in the gelatin/ $x$-carrageenan mixture. Biomacromolecules, 6, 14-23. http://doi.org/10.1021/bm0400473

Kasapis, S., \& Tay, S. L. (2009). Morphology of Molecular Soy Protein Fractions in Binary Composite Gels. Langmuir, 25(15), 8538-8547. http://doi.org/10.1021/la803290j

Katopo, L., Kasapis, S., \& Hemar, Y. (2012). Segregative phase separation in agarose/whey protein systems induced by sequence-dependent trapping and change in $\mathrm{pH}$. Carbobydrate Polymers, 87(3), 2100-2108. http://doi.org/10.1016/j.carbpol.2011.10.034

Kinsella, J. E. (1979). Functional properties of soy proteins. Journal of the American Oil Chemists' Society, 56(3), 242-258. http://doi.org/10.1007/BF02671468

Krintiras, G. A., Gadea Diaz, J., van der Goot, A. J., Stankiewicz, A. I., \& Stefanidis, G. D. (2016). On the use of the Couette Cell technology for large scale production of textured soy-based meat replacers. Journal of Food Engineering, 169, 205-213. http://doi.org/10.1016/j.jfoodeng.2015.08.021

Krintiras, G. A., Göbel, J., Bouwman, W. G., van der Goot, A. J., \& Stefanidis, G. D. (2014). On characterization of anisotropic plant protein structures. Food \& Function, 5(12), 3233-3240. http://doi.org/10.1039/c4fo00537f

Krintiras, G. A., Göbel, J., van der Goot, A. J., \& Stefanidis, G. D. (2015). Production of structured soybased meat analogues using simple shear and heat in a Couette Cell. Journal of Food Engineering, 160, 34-41. http://doi.org/10.1016/j.jfoodeng.2015.02.015

Ku, H., Wang, H., Pattarachaiyakoop, N., \& Trada, M. (2011). A review on the tensile properties of natural fiber reinforced polymer composites. Composites Part B: Engineering, 42(4), 856-873. 
http://doi.org/10.1016/j.compositesb.2011.01.010

Kweldam, A. C. (2011). Method for the preparation of a meat substitute product, meat substitute product obtained with the method and rady to consume meat sustitute product. http://doi.org/10.1016/j.(73)

Laestadius, L. I., Neff, R. A., Barry, C. L., \& Frattaroli, S. (2013). Meat consumption and climate change: The role of non-governmental organizations. Climatic Change, 120(1-2), 25-38. http://doi.org/10.1007/s10584-013-0807-3

Lam, M., Paulsen, P., \& Corredig, M. (2008). Interactions of Soy Protein Fractions with High-Methoxyl Pectin. Journal of Agricultural and Food Chemistry, 56, 4726-4735.

Lam, M., Shen, R., Paulsen, P., \& Corredig, M. (2007). Pectin stabilization of soy protein isolates at low pH. Food Research International, 40, 101-110. http://doi.org/10.1016/j.foodres.2006.08.004

Landon, G., Lewis, G., \& Boden, G. (1977). The influence of particle size on the tensile strength of particulate-filled polymers. Journal of Materials Science, 12, 1605-1613.

Lau, J. M., McNeil, M., Darvill, A. G., \& Albersheim, P. (1985). Structure of the backbone of rhamnogalacturonan I, a pectic polysaccharide in the primary cell walls of plants. Carbobydrate Research, 137, 111-125. http://doi.org/10.1016/0008-6215(85)85153-3

Ledward, D. a, \& Tester, R. F. (1994). Molecular transformations of proteinaceous foods during extrusion processing. Trends in Food Science \& Technology, 5(4), 117-120.

Lerdwijitjarud, W., Sirivat, A., \& Larson, R. G. (2002). Influence of elasticity on dispersed-phase droplet size in immiscible polymer blends in simple shearing flow. Polymer Engineering and Science, 42(4), 798809. http://doi.org/10.1002/pen.10992

Li, P. X., Campanella, O. H., \& Hardacre, A. K. (2004). Using an In-Line Slit-Die Viscometer to Study the Effects of Extrusion Parameters on Corn Melt Rheology. Cereal Chemistry, 81(1), 70-76. http://doi.org/10.1094/cchem.2004.81.1.70

Lin, S., Huff, H. E., \& Hsieh, F. (2000). Texture and Chemical Characteristics of Soy Protein Meat Analog Extruded at High Moisture. Food Chemistry and Toxocology, 65(2), 264-269.

Lin, S., Huff, H. E., \& Hsieh, F. (2002). Extrusion Process Parameters, Sensory Characteristics, and Structural Properties of a High Moisture Soy Protein Meat Analog. Journal of Food Science, 67(3), 10661072. http://doi.org/10.1111/j.1365-2621.2002.tb09454.x

Liu, K., \& Hsieh, F.-H. (2008). Protein-protein interactions during high-moisture extrusion for fibrous meat analogues and comparison of protein solubility methods using different solvent systems. Journal of Agricultural and Food Chemistry, 56(8), 2681-7. http://doi.org/10.1021/jf073343q

Liu, K. S., \& Hsieh, F.-H. (2007). Protein-protein interactions in high moisture-extruded meat analogs and heat-induced soy protein gels. Journal of the American Oil Chemists' Society, 84(8), 741-748. http://doi.org/10.1007/s11746-007-1095-8

Lopez de Ogara, M. C., Bercovich, F., Pilosof, A. M. R., \& Bartholomai, G. (1986). Denaturation of soybean proteins related to functionality and performance in a meat system. International Journal of Food Science \& Technology, 21(3), 279-287. http://doi.org/10.1111/j.1365-2621.1986.tb00407.x

Luisa, M., Freitas, F., Albano, K. M., Regina, V., \& Telis, N. (2017). Characterization of biopolymers and soy protein isolate-high-methoxyl pectin complex, 27(1), 62-67. 
Lundell, C., de Hoog, E. H. a, Tromp, R. H., \& Hermansson, A.-M. (2005). Effects of confined geometry on phase-separated dextran/gelatine mixtures exposed to shear. Journal of Colloid and Interface Science, 288(1), 222-9. http://doi.org/10.1016/j.jcis.2005.02.063

Lyu, S.-P., Bates, F. S., \& Macosko, C. W. (2000). Coalescence in polymer blends during shearing. Fluid Mechanics and Transport Phenomena, 46(2), 229-238. http://doi.org/10.1002/aic.690460203

Malav, O. P., Talukder, S., Gokulakrishnan, P., \& Chand, S. (2015). Meat Analog: A Review. Critical Reviews in Food Science and Nutrition, 55(9), 1241-1245. http://doi.org/10.1080/10408398.2012.689381

Maltais, A., Remondetto, G. E., \& Subirade, M. (2009). Soy protein cold-set hydrogels as controlled delivery devices for nutraceutical compounds. Food Hydrocolloids, 23(7), 1647-1653. http://doi.org/10.1016/j.foodhyd.2008.12.006

Manski, J. M., van der Goot, A. J., \& Boom, R. M. (2007a). Advances in structure formation of anisotropic protein-rich foods through novel processing concepts. Trends in Food Science \& Technology, 18(11), $546-557$.

Manski, J. M., van der Goot, A. J., \& Boom, R. M. (2007b). Formation of fibrous materials from dense calcium caseinate dispersions. Biomacromolecules, 8(4), 1271-1279.

Manski, J. M., van der Zalm, E. E. J., van der Goot, A. J., \& Boom, R. M. (2008). Influence of process parameters on formation of fibrous materials from dense calcium caseinate dispersions and fat. Food Hydrocolloids, 22(4), 587-600. http://doi.org/10.1016/j.foodhyd.2007.02.006

McAfee, A. J., McSorley, E. M., Cuskelly, G. J., Moss, B. W., Wallace, J. M. W., Bonham, M. P., \& Fearon, A. M. (2010). Red meat consumption: An overview of the risks and benefits. Meat Science, 84(1), 1 13. http://doi.org/10.1016/j.meatsci.2009.08.029

Micha, R., Wallace, S. K., \& Mozaffarian, D. (2010). Red and processed meat consumption and risk of incident coronary heart disease, stroke, and diabetes mellitus: A systematic review and meta-analysis. Circulation, 121(21), 2271-2283. http://doi.org/10.1161/CIRCULATIONAHA.109.924977

Mogensen, L., Hermansen, J. E., Halberg, N., Dalgaard, R., Vis, J. C., \& Smith, B. G. (2009). Sustainability in the food industry. In Life cycle assessment across the food supply chain (pp. 115-144).

Morley, J. G. (1976). New types of reinforced composite materials. Physics Reports, 28(3), 245-302. http://doi.org/10.1016/0370-1573(76)90033-8

Morris, E. R. (1992). The effect of solvent partition on the mechanical properties of biphasic biopolymer gels: an approximate theoretical treatment. Carbobydrate Polymers, 17(1), 65-70. http://doi.org/10.1016/0144-8617(92)90024-K

Morris, E. R. (2009). Functional interactions in gelling biopolymer mixtures. In Modern Biopolymer Science (First Edit, pp. 167-198). Elsevier. http://doi.org/10.1016/B978-0-12-374195-0.00005-7

Mulder, W., Peet-Schwering, C. van der, Hua, N.-P., \& Ree, R. van. (2016). Proteins for Food, Feed and Biobased Applications. Retrieved from www.iea-bioenergy.task42-biorefineries.com

Nagmani, B., \& Prakash, J. (1997). Functional properties of thermally treated legume flours. International Journal of Food Sciences and Nutrition, 48(3), 205-214. http://doi.org/10.3109/09637489709012594

Narayana, K., \& Narasinga Rao, M. S. (1982). Functional Properties of Raw and Heat Processed Winged Bean (Psophocarpus tetragonolobus) Flour. Journal of Food Science, 47(5), 1534-1538. 
http://doi.org/10.1111/j.1365-2621.1982.tb04976.x

Nijdam, D., Rood, T., \& Westhoek, H. (2012). The price of protein: Review of land use and carbon footprints from life cycle assessments of animal food products and their substitutes. Food Policy, 37(6), 760-770. http://doi.org/10.1016/j.foodpol.2012.08.002

Norton, I. T., \& Frith, W. J. (2001). Microstructure design in mixed biopolymer composites. Food Hydrocolloids, 15(4-6), 543-553. http://doi.org/10.1016/S0268-005X(01)00062-5

Onimawo, I. A., \& Akpojovwo, A. E. (2006). Toasting (dry heat) and nutrient composition, functional properties and antinutritional factors of pigeon pea (Cajanus cajan) flour. Journal of Food Processing and Preservation, 30(6), 742-753. http://doi.org/10.1111/j.1745-4549.2006.00093.x

Osen, R., \& Schweiggert-Weisz, U. (2016). High-moisture extrusion : meat analogues. In Reference Module in Food Science (pp. 1-7). Retrieved from https://doi.org/10.1016/B978-0-08-100596-5.03099-7

Osen, R., Toelstede, S., Eisner, P., \& Schweiggert-Weisz, U. (2015). Effect of high moisture extrusion cooking on protein-protein interactions of pea (Pisum sativum L.) protein isolates. International Journal of Food Science \& Technology, 50(6), 1390-1396. http://doi.org/10.1111/ijfs.12783

Otto, K. N., \& Wood, K. L. (1998). Product Evolution: A Reverse Engineering and Redesign Methodology. Research in Engineering Design, 10(4), 226-243. http://doi.org/10.1007/s001639870003

Pearson, A. M. (2012). Composition and structure. In Meat and muscle biochemistry (pp. 1-33).

Peighambardoust, S. H., Hamer, R. J., Boom, R. M., \& van der Goot, A. J. (2008). Migration of gluten under shear flow as a novel mechanism for separating wheat flour into gluten and starch. Journal of Cereal Science, 48(2), 327-338. http://doi.org/10.1016/j.jcs.2007.10.005

Peighambardoust, S. H., van Brenk, S., van der Goot, A. J., Hamer, R. J., \& Boom, R. M. (2007). Dough processing in a Couette-type device with varying eccentricity: Effect on glutenin macro-polymer properties and dough micro-structure. Journal of Cereal Science, 45(1), 34-48. http://doi.org/10.1016/j.jcs.2006.05.009

Peighambardoust, S. H., van der Goot, A. J., Hamer, R. J., \& Boom, R. M. (2004). A new method to study simple shear processing of wheat gluten-starch mixtures. Cereal Chemistry, 81(6), 714-721. Retrieved from http://dx.doi.org/10.1094/CCHEM.2004.81.6.714

Peters, J. P. C. M., Vergeldt, F. J., Boom, R. M., \& van der Goot, A. J. (2017). Water-binding capacity of protein-rich particles and their pellets. Food Hydrocolloids, 65, 144-156. http://doi.org/10.1016/j.foodhyd.2016.11.015

Petruccelli, S., \& Añón, M. C. (1994). Relationship between the Method of Obtention and the Structural and Functional Properties of Soy Protein Isolates. 1. Structural and Hydration Properties. Journal of Agricultural and Food Chemistry, 42(10), 2161-2169. http://doi.org/10.1021/jf00046a017

Pette, D., \& Staron, R. S. (1990). Cellular and molecular diversities of mammalian skeletal muscle fibers. Rev.Physiol Biochem.Pharmacol. (Vol. 116). http://doi.org/10.1007/3540528806

Pickering, K. L., Efendy, M. G. A., \& Le, T. M. (2016). A review of recent developments in natural fibre composites and their mechanical performance. Composites Part A: Applied Science and Manufacturing, 83, 98-112. http://doi.org/10.1016/j.compositesa.2015.08.038

Pietsch, V. L., Emin, M. A., \& Schuchmann, H. P. (2016). Process conditions influencing wheat gluten polymerization during high moisture extrusion of meat analog products. Journal of Food Engineering, 
198, 28-35. http://doi.org/10.1016/j.jfoodeng.2016.10.027

Post, M. J., \& Hocquette, J. F. (2017). New Sources of Animal Proteins; In Vitro Meat. New Aspects of Meat Quality - from genes to ethics. Elsevier Ltd. http://doi.org/10.1016/B978-0-08-100593-4/00017-5

Provencher, S. W. (1982). Contin: a general purpose constrained regularization program for inverting noisy linear algebraic and integral equations. Computer Physics Communications, 27, 229-242.

Puyvelde, P. V. Van, \& Moldenaers, P. (2005). Rheology and Morphology Development in Immiscible Polymer Blends. Rheology Reviews, 2005, 101-145. http://doi.org/10.1.1.361.7276

Ranasinghesagara, J., Hsieh, F. H., Huff, H., \& Yao, G. (2009). Laser scanning system for real-time mapping of fiber formations in meat analogues. Journal of Food Science, 74(2). http://doi.org/10.1111/j.17503841.2008.01032.x

Ranasinghesagara, J., Hsieh, F., \& Yao, G. (2006). A photon migration method for characterizing fiber formation in meat analogs. Journal of Food Science, 71(5). http://doi.org/10.1111/j.17503841.2006.00038.x

Rehrah, D., Ahmedna, M., Goktepe, I., \& Yu, J. (2009). Extrusion parameters and consumer acceptability of a peanut-based meat analogue. International Journal of Food Science \& Technology, 44(10), 2075-2084. http://doi.org/10.1111/j.1365-2621.2009.02035.x

Reig, M., Lillford, P. J., \& Toldrá, F. (2008). Structured meat products. Food Materials Science: Principles and Practice, 501-523. http://doi.org/10.1007/978-0-387-71947-4_21

Renard, C. M. G. C., \& Thibault, J. F. (1996). Degradation of pectins in alkaline conditions: Kinetics of demethylation. Carbohydrate Research, 286, 139-150. http://doi.org/10.1016/0008-6215(96)00056-0

Ruiz De Huidobro, F., Miguel, E., Blázquez, B., \& Onega, E. (2005). A comparison between two methods (Warner-Bratzler and texture profile analysis) for testing either raw meat or cooked meat. Meat Science, 69(3), 527-536. http://doi.org/10.1016/j.meatsci.2004.09.008

Russin, T. A., Boye, J. I., Arcand, Y., \& Rajamohamed, S. H. (2011). Alternative Techniques for Defatting Soy: A Practical Review. Food and Bioprocess Tecbnology, 4(2), 200-223. http://doi.org/10.1007/s11947-010-0367-8

Sadeghi, F., Kadkhodaee, R., Emadzadeh, B., \& Phillips, G. O. (2018). Phase behavior, rheological characteristics and microstructure of sodium caseinate-Persian gum system. Carbohydrate Polymers, 179(July 2017), 71-78. http://doi.org/10.1016/j.carbpol.2017.09.060

Sahu, S., \& Broutman, L. J. (1972). Mechanical properties of particulate composites. Polymer Engineering and Science, 12(2), 91-100.

Schmidt, J. W., Agga, G. E., Bosilevac, J. M., Brichta-Harhay, D. M., Shackelford, S. D., Wang, R., ... Arthur, T. M. (2015). Occurrence of antimicrobial-resistant Escherichia coli and Salmonella enterica in the beef cattle production and processing continuum. Applied and Environmental Microbiology, 81(2), 713-725. http://doi.org/10.1128/AEM.03079-14

Scholten, E., Tuinier, R., Tromp, R. H., \& Lekkerkerker, H. N. W. (2002). Interfacial tension of a decomposed biopolymer mixture. Langmuir, 18(12), 2234-2238. http://doi.org/10.1021/la0114373

Schösler, H., de Boer, J., \& Boersema, J. J. (2012). Can we cut out the meat of the dish? Constructing consumer-oriented pathways towards meat substitution. Appetite, 58(1), 39-47. 
http://doi.org/10.1016/j.appet.2011.09.009

Seiichiro, I., \& Noguchi, A. (1987). High moisture extrusion with twin screw extruder - fate of soy protein during the repetition of extrusion cooking.pdf. Nippon Shokubin Kogyo Gakkaishi, 34(7), 456-461.

Shpigelman, A., Kyomugasho, C., Christiaens, S., Van Loey, A. M., \& Hendrickx, M. E. (2014). Thermal and high pressure high temperature processes result in distinctly different pectin non-enzymatic conversions. Food Hydrocolloids, 39, 251-263. http://doi.org/10.1016/j.foodhyd.2014.01.018

Shrinivas, P., Kasapis, S., \& Tongdang, T. (2009). Morphology and mechanical properties of bicontinuous gels of agarose and gelatin and the effect of added lipid phase. Langmuir, 25(2), 8763-8773. http://doi.org/10.1021/la9002127

Simeone, M., Alfani, A., \& Guido, S. (2004). Phase diagram, rheology and interfacial tension of aqueous mixtures of Na-caseinate and Na-alginate. Food Hydrocolloids, 18(3), 463-470. http://doi.org/10.1016/j.foodhyd.2003.08.004

Simeone, M., Sibillo, V., Tassieri, M., \& Guido, S. (2002). Shear-induced clustering of gelling droplets in aqueous biphasic mixtures of gelatin and dextran. Journal of Rheology, 46(5), 1263. http://doi.org/10.1122/1.1501962

Singh, P., Kumar, R., Sabapathy, S. N., \& Bawa, A. S. (2008). Functional and edible uses of soy protein products. Comprehensive Reviens in Food Science and Food Safety, 7(1), 14-28. http://doi.org/10.1111/j.1541-4337.2007.00025.x

Smetana, S., Mathys, A., Knoch, A., \& Heinz, V. (2015). Meat alternatives: life cycle assessment of most known meat substitutes. The International Journal of Life Cycle Assessment, 20(9), 1254-1267. http://doi.org/10.1007/s11367-015-0931-6

Steinfeld, H., Gerber, P., Wassenaar, T. D., Castel, V., \& de Haan, C. (2006). Livestock's long shadow: environmental issues and options. (Food \& Agriculture Org, Ed.).

Stiftung, Böll, H., \& Terre, A. de la. (2014). Meat atlas: facts and figures about the animals we eat. Heinrich Böll Foundation and Friends of the Earth Europe (Vol. 68). Retrieved from https://www.foeeurope.org/sites/default/files/publications/foee_hbf_meatatlas_jan2014.pdf

Stokes, J. R., Wolf, B., \& Frith, W. J. (2001). Phase-separated biopolymer mixture rheology: Prediction using a viscoelastic emulsion model. Journal of Rheology, 45(5), 1173-1191. http://doi.org/10.1122/1.1389314

Sturtewagen, L., De Soete, W., Dewulf, J., Lachat, C., Lauryssen, S., Heirman, B., ... Schaubroeck, T. (2016). Resource use profile and nutritional value assessment of a typical Belgian meal, catered or home cooked, with pork or Quorn ${ }^{\mathrm{TM}}$ as protein source. Journal of Cleaner Production, 112, 196-204. http://doi.org/10.1016/j.jclepro.2015.09.006

Sui, Z., Raubenheimer, D., \& Rangan, A. (2017). Exploratory analysis of meal composition in Australia: Meat and accompanying foods. Public Health Nutrition, 20(12), 2157-2165. http://doi.org/10.1017/S1368980017000982

Takayanagi, M., Harima, H., \& Iwata, Y. (1963). Viscoelastic behavior of polymer blends and its comparison with model experiments. The Society of Materials Science, 12(116), 389-394.

Taylor, G. I. (1932). The viscosity of a fluid containing small drops of another fluid. Proceedings of the Royal Society A: Mathematical, Physical and Engineering Sciences, 138(834), 41-48. http://doi.org/10.1098/rspa.1932.0169 
Taylor, G. I. (1934). The formation of emulsions in definable fields of flow. Proceedings of the Royal Society A: Mathematical, Physical and Engineering Sciences, 146(858), 501-523. http://doi.org/10.1098/rspa.1934.0169

Thakur, B. R., Singh, R. K., \& Handa, A. K. (1997). Chemistry and uses of pectin--a review. Critical Reviews in Food Science and Nutrition, 37(1), 47-73. http://doi.org/10.1080/10408399709527767

Tolstoguzov, V. B. (1988). Some physico-chemical aspects of protein processing into foodstuffs. Food Hydrocolloids, 2(5), 339-370. http://doi.org/10.1016/S0268-005X(88)80001-8

Tolstoguzov, V. B. (1991). Functional properties of food proteins and role of protein-polysaccharide interaction. Food Hydrocolloids, 4(6), 429-468. http://doi.org/10.1016/S0268-005X(09)80196-3

Tolstoguzov, V. B. (1998). Functional properties of protein-polysaccharide. In Functional properties of food macromolecules (pp. 252-275).

Tolstoguzov, V. B., Mzhel'sky, A. I., \& Gulov, V. Y. (1974). Deformation of emulsion droplets in flow. Colloid \& Polymer Science, 252, 124-132.

United Nations - Department of Economic and Social Affairs. (2015). World population prospects: The 2015 Revision.

van den Einde, R. M., van der Goot, a. J., \& Boom, R. M. (2003). Understanding molecular weight reduction of starch during heating - shearing processes. Journal of Food Science, 68(8), 2396-2404. http://doi.org/10.1111/j.1365-2621.2003.tb07036.x

Verbeke, W., Van Wezemael, L., de Barcellos, M. D., Kügler, J. O., Hocquette, J. F., Ueland, O., \& Grunert, K. G. (2010). European beef consumers' interest in a beef eating-quality guarantee. Insights from a qualitative study in four EU countries. Appetite, 54(2), 289-296. http://doi.org/10.1016/j.appet.2009.11.013

Verhulst, K., Cardinaels, R., Moldenaers, P., Afkhami, S., \& Renardy, Y. (2009). Influence of viscoelasticity on drop deformation and orientation in shear flow. Part 2: Dynamics. Journal of Non-Newtonian Fluid Mechanics, 156(1-2), 44-57. http://doi.org/10.1016/j.jnnfm.2008.10.003

Vermant, J. (2001). Large-scale structures in sheared colloidal dispersions. Current Opinion in Colloid and Interface Science, 6(5-6), 489-495. http://doi.org/10.1016/S1359-0294(01)00117-0

Vliet, T. van. (1996). Large deformation and fracture behaviour of gels. Current Opinion in Colloid \& Interface Science, 1(6), 740-745. http://doi.org/10.1039/fd9950100359

Vogel, R. H. (1988). SPLMOD Users Manual, Data Analysis Group, EMBL-DA09, EMBL.".

Walstra, P. (2004). Physical Chemistry of Foods. European Journal of Pharmaceutics and Biopharmaceutics (Vol. 57). http://doi.org/10.1016/j.ejpb.2003.10.013

Weiss, G. H., Porra, J. M., \& Masoliver, J. (1998). The continuous - time random walk description of photon motion in an isotropic medium. Optics Communications, 146(January), 268-276. http://doi.org/10.1016/S0030-4018(97)00475-6

Wildes, G., Keskkula, H., \& Paul, D. R. (1999). Coalescence in PC/SAN blends: Effect of reactive compatibilization and matrix phase viscosity. Polymer, 40(20), 5609-5621. http://doi.org/10.1016/S0032-3861(98)00776-9 
Wolf, B. (2013). Designed Food Structures Based on Hydrocolloids. In J. E. Norton, P. J. Fryer, \& I. T. Norton (Eds.), Formulation Engineering of Foods (First Edit, pp. 59-81). Chichester, UK: John Wiley \& Sons, Ltd.

Wolf, B., \& Frith, W. J. (2003). String phase formation in biopolymer aqueous solution blends. Journal of Rheology, 47(5), 1151. http://doi.org/10.1122/1.1603238

Wolf, B., Scirocco, R., Frith, W. J., \& Norton, I. T. (2000). Shear-induced anisotropic microstructure in phase-separated biopolymer mixtures. Food Hydrocolloids, 14, 217-225. http://doi.org/10.1016/S0268-005X(99)00062-4

Wu, J., \& Meredith, J. C. (2014). Assembly of chitin nanofibers into porous biomimetic structures via freeze drying. ACS Macro Letters, 3(2), 185-190. http://doi.org/10.1021/mz400543f

Wu, V. Y., \& Inglett, G. E. (1974). Denaturation of plant proteins related to functionaltity and food applications. Journal of Food Science, 39(2), 218-225.

Yao, G., Liu, K.S., and Hsieh, F. (2004). A new method for characterizing fiber formation in meat analogs during high-moisture extrusion. Journal of Food Science, 69(7), 303-307.

Yoo, S. H., Fishman, M. L., Hotchkiss, A. T., \& Hyeon, G. L. (2006). Viscometric behavior of highmethoxy and low-methoxy pectin solutions. Food Hydrocolloids, 20(1), 62-67. http://doi.org/10.1016/j.foodhyd.2005.03.003

Zalm, E. E. J. van der, Goot, A. J. van der, \& Boom, R. M. (2009). Influence of process conditions on the separation behaviour of starch-gluten systems. Journal of Food Engineering, 95(4), 572-578. http://doi.org/10.1016/j.jfoodeng.2009.06.038

Zayas, J. F. (1997). Solubility of proteins. In Functionality of proteins in food (pp. 6-75). Berlin, Heidelberg: Springer. 
Summary 
A transition from animal to plant-based protein is required to produce sufficient protein for the growing world population, while at the same time mitigates climate change. Especially the production of meat imposes a burden on the environment. Meat analogues, which are products that are similar to meat in its functionality, can help consumers to lower their meat consumption. The anisotropic, fibrous nature of meat is perhaps the most important characteristic of meat, which can be mimicked by structuring biopolymers, such as proteins and polysaccharides with the shear cell technology. The aim of this thesis is to obtain insight in the key mechanisms that play a role in the transformation of plant-based biopolymer blends into anisotropic/fibrous structures with shear cell technology. These two key mechanisms are the deformation of the two phases present in biopolymer blends, and the subsequent entrapment of this deformation during solidification. It was concluded that successful structure formation requires matching of the properties of the two phases. During structuring at elevated temperature, the two phases are deformed, while subsequent cooling ensures entrapment of the deformed dispersed phase(s) in the (continuous) phase. Ideally, the continuous and dispersed phase have different strength in the final product,.

Chapter 2 presents a method to determine the water distribution in soy protein isolate (SPI) wheat gluten (WG) blends. The concentration of water in each separate phase was directly determined with time-domain nuclear magnetic resonance relaxometry (TD-NMR), and oscillatory rheology was used to indirectly asses the water distribution by determining the viscoelastic properties of the separate phases and the blend. It was shown that water distributes unevenly in SPI-WG blends: more water was absorbed by the SPI as compared to the WG phase. This methodology was developed for SPI-WG blends at room temperature and subsequently also applied to heated and sheared samples in Chapter 3. First, water distribution in the blend after a heat and/or shear treatment was assessed with TD-NMR and the outcomes were then used to predict the viscoelastic properties of the SPI and WG phase in the blend. This yielded insight in the deformability of the two phases in the blend. The viscoelastic properties were measured under conditions that are relevant for structure formation, i.e. during and after heating and shearing. It was shown that the water distribution was hardly affected by a heat or shear treatment, whereas the viscoelastic properties of the two phases changed significantly. The viscoelastic properties of SPI and WG became more similar due to water redistribution in the blend, which allows deformation and alignment of the dispersed phase during structuring. 
Chapter 4 describes a study using a model blend that mimics soy protein concentrate (SPC). It consists of a relatively pure protein phase, soy protein isolate (SPI), and a soluble, more or less pure polysaccharide phase, pectin. This SPI-pectin blend formed fibrous materials at a similar heating temperature as SPC, being $140^{\circ} \mathrm{C}$. Pectin formed the dispersed phase and was deformed when heated and sheared at optimal conditions. Chapter $\mathbf{5}$ extends the study on structure formation with SPI-pectin blends. Here, the deformation of the dispersed pectin phase and the influence of incorporated air were considered. The fibrous nature of these products appears upon tearing, and originates from detachment through or along the long side of the weak dispersed phase(s), being pectin and/or air. A model based on the rule of mixing was used to predict the mechanical anisotropy based on the volume fraction and the deformation of the weak, dispersed phase. The size and orientation of the dispersed phases, tailored by using different shear rates, were related to differences in fracture behavior when deforming the structures. Besides deformation, the strength and volume fraction of the weak phase(s) were important when composing a blend for fibrous structure formation. In Chapter $\mathbf{6}$, the behavior of the SPI and pectin phases in a blend was investigated by determining the viscoelastic properties while shearing and heating over time. A closed cavity rheometer (CCR) was used to determine these properties under similar conditions as used during fibrous structure formation. The addition of a small amount of pectin (2.2 wt.\%) to a SPI dispersion (41.8 wt.\%) resulted in viscoelastic behavior that changed in time during a shear treatment at elevated temperatures. Although one can clearly discern two distinct phases with SEM, the viscoelastic behavior of the SPI-pectin blend is more complex than that of a simple composite material.

Chapter 7 demonstrates the importance of the fractionation process on the structuring potential of soy proteins. An enriched soy protein fraction was obtained through an aqueous fractionation process. Those fractions could be used to make fibrous structures when: i) the soy protein fractions were toasted, which is a dry heating step, and ii) when a concentrate ( $75 \%$ protein) was combined with full fat flour, in such a ratio that the protein content was similar to commercial SPC. Toasting results in decreased protein solubility, increased water holding capacity and increased viscosity of the fractions, and these changes turned out to be important for fibrous structure formation. 
Lastly, literature was reviewed to put all findings in perspective (Chapter 8). An overview is presented of all techniques that are commercially used and currently investigated to create meatlike structures. Structuring techniques are compared in their approach, being either bottom-up, which refers to assembly of structural elements that are then combined, or top-down, which refers to structuring of biopolymer blends using an overall force field. A bottom-up strategy has the potential to resemble the structure of meat most closely, by structuring the molecules including proteins into structural components (e.g. muscle cells) followed by assembly of individual structural components. A top-down strategy is more efficient in its use of resources and is better scalable, but can only create the desired structure on larger length scales. The techniques with a top-down strategy were further investigated by reviewing literature on similar processes outside this particular field of application, i.e. not meant to create fibrous structures. These insights were subsequently translated to the conditions as used in structure formation for meat analogues.

Chapter 9 concludes with a general discussion of all results presented in this thesis. The different chapters are integrated in design rules for fibrous structure formation. Furthermore, the complexity encountered when studying material and conditions during fibrous structure formation are discussed. Then, the potential and the challenges for understanding and applying fibrous structure formation with simple shear flow are summarized.

The overall societal goal of developing meat analogue food products is to help consumers in the transition from animal-based to a more plant-based diet. The scientific goal to obtain insight in fibrous structure formation with the shear technology as developed in this thesis is of importance, and can be the basis for developing the technology for the next generation meat analogues. 


\section{Acknowledgements}

I would like to acknowledge all who were involved with the work presented in this thesis. First and foremost, I would like to thank my supervisors Atze Jan and Remko for allowing me the opportunity to work at the Laboratory of Food Process Engineering, for their dedication, enthusiasm, and originality. They have made the past four years a pleasant and memorable period. My gratitude goes to the technicians from FPE. Jarno thanks for all your help with the shear cell(s), microscopy and especially the (mostly) fun and sometimes somewhat stressful moments working with the large couette cell. Jos, thank you for all your help in the lab and for always helping me out, whatever the problem is. Martin and Maurice, it was a lot of fun to organize the $\mathrm{PhD}$ trip with you. Marjan and Ilona, I greatly appreciate your administrative support, sweets and chitchats.

Many others have contributed to my work during the past four years. I am thankful to all the men from the technical developments studio, who were always happy to optimize the shear cells, design and make new useful tools, or help when something broke down in the lab. Costas and Daan, thank you very much for helping me write the first scientific publications. Remco, Tiny, Norbert, Frank and Henk, thanks a lot for your help with analysing samples or analysing data. All my brilliant students, Nick, Tjachy, Bas, Luuk, Nicolien, Rik, Jelle, Martin, Lydie, Noortje, and Famke, I enjoyed every moment spend working with you and I hope you learned as much from me as I did from you. All the collaborators in the Plant Meat Matters Consortium, the past one and a half year was very inspiring and interesting for me, and I am very happy to stay part of this group for some more time. Thank you Jacqueline, it is great to work with you (again), I really enjoy the time we spend at Meyn to test the new versions of the shear cell. I had the great pleasure to collaborate with many PhDs and postdocs, Patricia, Yu, Wanqing, Jan, Steven, Ariane, Nynke, Floor, Konstantina and Elvira, thank you very much!

Thank you all the PhD colleagues and friends! Lu, Eline, Victor, Jue, Jorien, Zhulaj and Fiona, thank you for being great office-mates: discussing research, good ideas, listening to music (on Friday afternoon or any other day) and a lot of fun! Thank you Laura, Meinou, Marlies and Ties for making me feel at home and discussing all the perks of coming from Brabant. Angelica and $\mathrm{Qi}, \mathrm{I}$ did not forget about you as also being great organizers for the $\mathrm{PhD}$ trip to Germany and 
Switzerland, thank you for all the great meetings with nice food, drinks and cakes. I wish to thank all the active, athletic, and sportive colleagues, Anja, Evelien, Eline, Juliana, Dimitri, and many others, to spend lunch in the gym with, and I wish to thank all the adventurous ones, Emma, Steven, Jaap and Nynke for the great time in Canada. Thanks to my housemates, Patricia, Pina, Andrea (and Fiona), for the lovely dinners, drinks and talks.

My acknowledgements would be incomplete without thanking all my friends and family. All my girlfriends from Zeeland, thank you for your support and discussions about molecules! Thanks for the support from my friends from Kantjeboord, Maartje, Annelaura and Sanne, the A(B)C girls, Anneloes and Cho, and my EMFS family. Thank you mom and dad for always supporting me and making me feel I can do anything! Thank you Tessa for designing this beautiful cover. Thank you Marnick for all the nice discussions we had. A special thanks to Fiona and Anneloes for being my paranymphs. Although we only fell in love at the last stage of my thesis, Jaap, thank you for all your support and the joy you bring into my life. 


\section{About the author}

Birgit Dekkers was born on 14 May 1991 in Veghel, The Netherlands. She attended Udens College in Uden, where she obtained her VWO diploma in 2009, with a major in Natuur en Gezondheid (Nature and Health).

In 2009, Birgit started her study Nutrition and Health at Wageningen University with in the third year a minor Food Technology. In 2012, Birgit started the European Master in Food Studies, a Master programme in which you study at leading universities in four European countries. She was part

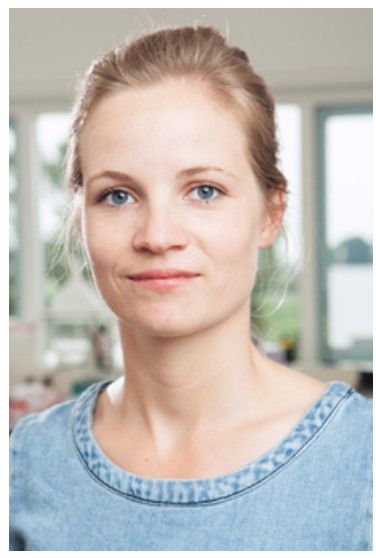
of a selected group of talented students who are privileged to receive training from top academic staff and respected experts in industry. The first year she completed different courses and studied in the Netherlands (Wageningen University \& Research), Ireland (University College Cork), France (AgroParisTech) and Sweden (Lund University). In the second year, she did a combined thesis internship project at Nestlé Research Centre in Lausanne (Switzerland). The project was entitled Encapsulation to regulate the release of bioactive lipids.

After completing her MSc studies, she continued working as a $\mathrm{PhD}$ candidate at the Food Process Engineering group of Wageningen University \& Research on the project Creation of fibrous plant protein products. 


\section{List of publications}

\section{This thesis:}

B. L. Dekkers, D.W. de Kort, K.J. Grabowska, B. Tian, H. van As, A.J. van der Goot (2016). A combined Rheology and Time Domain NMR approach for determining water distribution in Protein Blends. Food Hydrocolloids, 60, 525-532. http://doi.org/10.1017/CBO9781107415324.004

B.L. Dekkers, C.V. Nikiforidis, A.J. van der Goot (2016). Shear-induced fibrous structure formation from a pectin / SPI blend. Innovative Food Science and Emerging Technologies, 36, 193200. http://doi.org/10.1016/j.ifset.2016.07.003

M. E. J. Geerts, B. L. Dekkers, A. van der Padt, A.J. van der Goot (2017). Aqueous fractionation processes of soy protein for meat analogs. Innovative Food Science and Emerging Technologies, 45, 313-319. http://doi.org/10.1016/j.ifset.2017.12.002

B.L. Dekkers, M.A. Emin, R.M. Boom, A.J. van der Goot (2018). The phase properties of soy protein and wheat gluten in a blend for fibrous structure formation. Food Hydrocolloids, 79, 273 281. http://doi.org/10.1016/j.foodhyd.2017.12.033

B.L. Dekkers, R. Hamoen, R.M. Boom, A.J. van der Goot (2018). Understanding fiber formation in a concentrated soy protein isolate - Pectin blend. Journal of Food Engineering, 222, 84-92. http://doi.org/10.1016/j.jfoodeng.2017.11.014

B.L. Dekkers, R.M. Boom, A.J. van der Goot (2018). Viscoelastic properties of soy protein isolate - pectin blends: Richer than those of a simple composite material. Food Research International, 107, 281-288. http://doi.org/10.1016/j.foodres.2018.02.037

B.L. Dekkers, R.M. Boom, A.J. van der Goot (2018). Structuring processes for meat analogues. Submitted for publication 


\section{Other publications}

K.J. Grabowska, S. Zhu, B.L. Dekkers, N.C.A. de Ruijter, J. Gieteling, A.J. van Der Goot (2016). Shear-induced structuring as a tool to make anisotropic materials using soy protein concentrate. Journal of Food Engineering, 188, 77-86.

http://doi.org/10.1016/j.jfoodeng.2016.05.010

B.L. Dekkers, E. Kolodziejczyk, S. Acquistapace, J. Engmann, T.J. Wooster (2016). Impact of gastric $\mathrm{pH}$ profiles on the proteolytic digestion of mixed $\beta$ lg-Xanthan biopolymer gels. Food \& function, 7(1), 58-68.

B.L. Dekkers, A.J. van der Goot (2018). Chapter 21 - Novel processing concepts for making fibrous food products in Intensification of Biobased Processes, 462-477

T.J. Wooster, S. Acquistapace, C. Mettraux, L. Donato-Capel, B.L. Dekkers (2018). Hierarchically structured biopolymer hydrogels create tailorable delayed burst release during gastrointestinal digestion. Submitted for publication

B.L. Dekkers, S. Acquistapace, L. Donato, V. Soulie, R. Stoudmann, C. Loussert, C. Meyer, J. Engmann, T.J. Wooster (2018). Biodegradable biopolymer core-shell networks for programmable digestive burst release of encapsulated lipophilic active ingredients. Submitted for publication 


\section{Overview of completed training activities}

\section{Discipline specific activities}

\section{Courses}

Reaction kinetics in food sciences (Wageningen, The Netherlands) 2014

Capita selecta Biophysics: Time domain magnetic resonance in food materials 2015

(Wageningen, The Netherlands)

Advanced Food Analysis (Wageningen, The Netherlands) 2014

Numerical Methods for Chemical Engineers (Eindhoven, The Netherlands) 2015

European school on Rheology (Leuven, Belgium) 2015

SAS School (Utrecht, The Netherlands) 2015

\section{Conferences}

NPS14 (Utrecht, The Netherlands) ${ }^{1} \quad 2014$

The international Symposium On Food Rheology and Structure (Zürich, 2015 Switzerland) ${ }^{2}$

$2^{\text {nd }}$ Wageningen PhD symposium (Wageningen, The Netherlands) 2016

Food Structure Functionality Forum (Singapore, Singapore) ${ }^{2} 2016$

Food Colloids (Wageningen, The Netherlands) ${ }^{2} \quad 2016$

$3^{\text {rd }}$ Wageningen PhD symposium (Wageningen, The Netherlands) 2017

Delivery of Functionality (Auckland, New Zealand) ${ }^{1,2}$

$\begin{array}{ll}\text { IFST (Erding, Germany) }{ }^{2} & 2017\end{array}$

Food Structure Functionality Forum (Montreal, Canada) ${ }^{2} 2018$

International Food Technology IFT (Chicago, USA) ${ }^{1} \quad 2018$

\section{General courses}

$\begin{array}{ll}\text { VLAG PhD week } & 2015\end{array}$

$\begin{array}{ll}\text { Organizing \& supervising MSc. Thesis } & 2015\end{array}$

Process economics \& cost engineering (Eindhoven, The Netherlands) 2015

Techniques for writing and presenting a scientific paper 2016

$\begin{array}{ll}\text { Scientific writing } & 2017\end{array}$

$\begin{array}{ll}\text { Career assessment } & 2018\end{array}$ 


\section{Optional courses and activities}

Food Process Engineering Group day

2014-2017

VLAG PhD council

2016-2018

Wageningen $\mathrm{PhD}$ council

2016-2017

PhD study tour, Germany and Switzerland 1,2

2016

PhD study tour, Canada ${ }^{1,2}$

2018

${ }^{1}$ Poster presentation

${ }^{2}$ Oral presentation 

The work presented in this thesis has been carried out in the framework of the Institute of Sustainable Process Technology (ISPT) under the project "PI-00-03 IPS: Intensified Protein Structuring for More Sustainable Food". This research is part of the project Plant Meat Matters, which is co-financed by Top Consortium for Knowledge and Innovation Agri \& Food by the Dutch Ministry of Economic Affairs; the project is registered under contract number TKI-AF16011.

The cover was designed by Tessa Dekkers

Printed by: Digiforce || ProefschriftMaken 